\title{
LAYER GROWTH AND HYSTERESIS MODELLING \\ OF EPITAXIAL LEAD ZIRCONATE TITANATE THIN FILMS
}
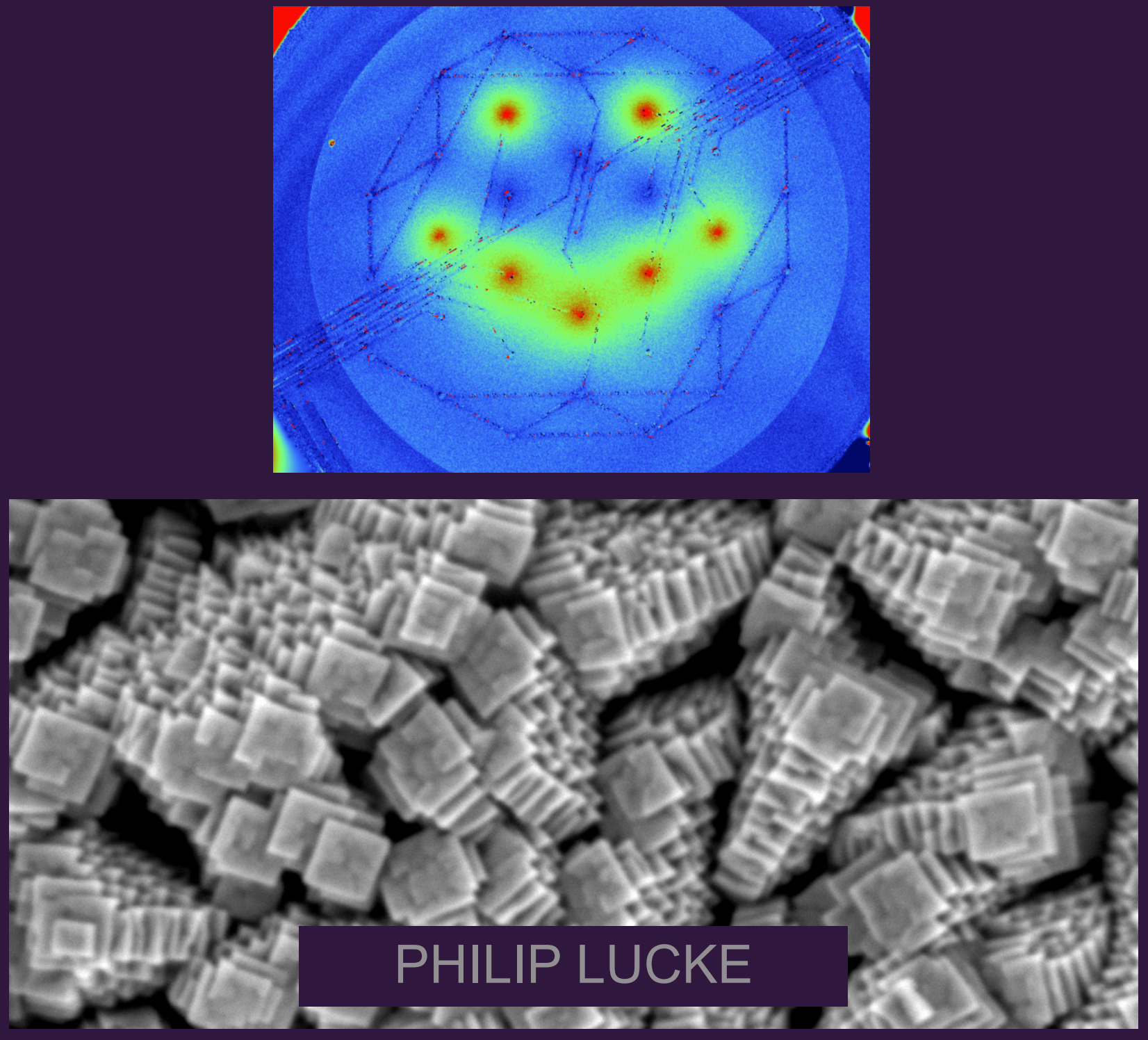


\section{LAYER GROWTH AND HYSTERESIS}

MODELLING OF EPITAXIAL LEAD ZIRCONATE TITANATE THIN FILMS 



\title{
LAYER GROWTH AND HYSTERESIS MODELLING OF EPITAXIAL LEAD ZIRCONATE TITANATE THIN FILMS
}

\section{DISSERTATION}

\author{
to obtain \\ the degree of doctor at the University of Twente, \\ on the authority of the rector magnificus, \\ prof. dr. ir. A. Veldkamp, \\ on account of the decision of the Doctorate Board, \\ to be publicly defended \\ on Wednesday 21 April 2021 at 14:45 hours \\ by \\ Philip Lucke
}

born on the $25^{\text {th }}$ of February 1990

in Moers, Germany 
This dissertation has been approved by:

Supervisor prof. dr. ir. F. Bijkerk

Co-supervisor dr. A.E. Yakshin

Co-supervisor dr. M. Bayraktar

\section{Graduation Committee:}

Chairman/secretary:

prof. dr. J. L. Herek

University of Twente, TNW

Supervisor:

prof. dr. ir. F. Bijkerk,

University of Twente, TNW

Co-supervisors:

dr. A.E. Yakshin

University of Twente, TNW

dr. M. Bayraktar

University of Twente, TNW

Committee Members:

prof. dr. B. Noheda

University of Groningen

dr. E. Defay

Luxembourg Institute of Science and Technology

prof. dr. ir. W. G. van der Wiel

prof. dr. ir. J. E. ten Elshof

University of Twente, TNW

dr. ir. E. P. Houwman

University of Twente, TNW

University of Twente, TNW

Keywords: PZT, ferroelectrics, piezoelectrics, adaptiv optics, hysteresis, thin film growth, ferroelectric loss, nonlinearity

Design: Cover art was designed by P. Lucke. It shows an optical profile generated by the demonstrator and a surface SEM of LNO grown on PZT (frontside),the backside shows a cross-sectional SEM of a PZT film with LNO deposited on top.

Printed by: Ipskamp Printing

ISBN: $\quad$ 978-90-365-5152-6

DOI: $\quad 10.3990 / 1.9789036551526$ 
Copyright (C) 2021 by P. Lucke, The Netherlands. All rights reserved. No parts of this thesis may be reproduced, stored in a retrieval system or transmitted in any form or by any means without permission of the author.

Alle rechten voorbehouden. Niets uit deze uitgave mag worden vermenigvuldigd, in enige vorm of op enige wijze, zonder voorafgaande schriftelijke toestemming van de auteur.

Acknowledgments:

This work is part of the research programme "Smart Multilayer Interactive Optics for Lithography at Extreme UV wavelength (SMILE)" with contract number 10448 and has received funding from the ECSEL Joint Undertaking (JU) under grant agreement No 826422. The JU receives support from the European Union's Horizon 2020 research and innovation programme and Netherlands, Belgium, Germany, France, Romania, Israel. SMILE is financially supported by the Nederlandse Organisatie voor Wetenschappelijk Onderzoek (NWO) and Carl Zeiss SMT. This research has been carried out in the Industrial Focus Group XUV Optics, being part of the MESA+ Institute for Nanotechnology and the University of Twente (http://www.utwente.nl/xuv). The Industrial Focus Group XUV Optics receives further support from the Province of Overijssel, ASML and Malvern Panalytical.

\section{UNIVERSITY $\mid$ MESA+
OF TWENTE.}
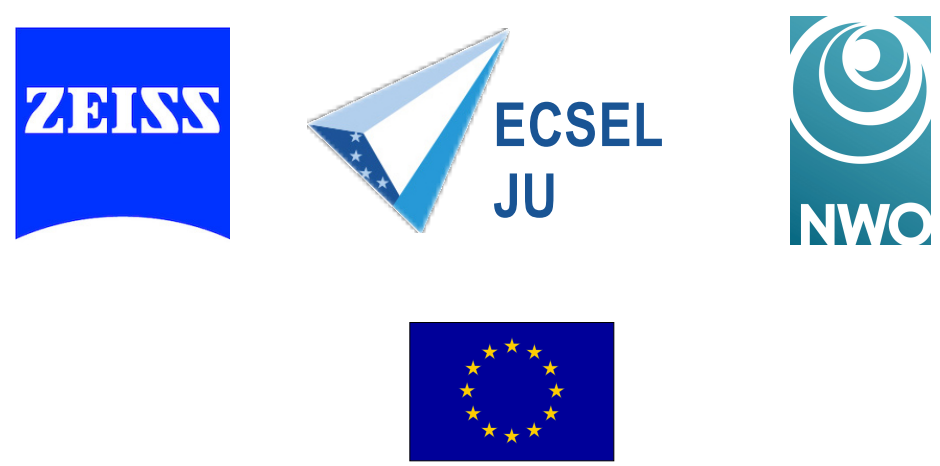

To my family and partner Maike who always supported me. 



\section{List of Publications}

This thesis is based on a patent and the following publications:

Chapter 3: M. Nematollahi, P. Lucke, M. Bayraktar, A.E. Yakshin, G. Rijnders, and F. Bijkerk, "Nanoscale piezoelectric surface modulation for adaptive extreme ultraviolet and soft x-ray optics", Optics Letters, 44, 51045107 (2019)

Chapter 4: P. Lucke, M. Nematollahi, M. Bayraktar, A.E. Yakshin,, J.E. ten Elshof, and F. Bijkerk, "Influence of template layer on structure and ferroelectric properties of $\mathrm{PbZr}_{0.52} \mathrm{Ti}_{0.48} \mathrm{O}_{3}$ films", submitted to ACS Applied Electronic Materials

Chapter 5: P. Lucke, M.Bayraktar, Y.A. Birkhölzer, M. Nematollahi, A.E. Yakshin, G. Rijnders, F. Bijkerk and E.P. Houwman, "Hysteresis, loss and nonlinearity in epitaxial $\mathrm{PbZr}_{0.55} \mathrm{Ti}_{0.45} \mathrm{O}_{3}$ films: A polarization rotation model", Advanced Functional Materials, 2020, 2005397

Chapter 6: P. Lucke, M.Bayraktar, N. Schukkink, A.E. Yakshin, G. Rijnders, F. Bijkerk and E.P. Houwman, "Hysteresis, loss and nonlinearity in epitaxial $\mathrm{PbZr}_{0.55} \mathrm{Ti}_{0.45} \mathrm{O}_{3}$ films: Polarization rotation under a $\mathrm{DC}$ bias field", submitted to Advanced Electronic Materials

T. Gruner, K. Hild, J. Lippert, P. Lucke and M. Nematollahi, German Patent (2020) 



\section{Contents}

List of Publications $\quad 9$

1 Introduction $\quad \mathbf{1 5}$

1.1 Motivation and outlook . . . . . . . . . . . . . . . . 16

1.2 Thesis outline. . . . . . . . . . . . . . . . . . 19

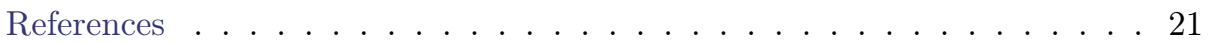

2 Experimental $\quad 27$

2.1 Thin film fabrication . . . . . . . . . . . . . . . . . . 28

2.2 Characterization . . . . . . . . . . . . . . . . . . 29

2.2.1 Crystal structure . . . . . . . . . . . . . . . . 29

2.2.2 Surface and microstructure. . . . . . . . . . . . . . 30

2.2.3 Functional properties................ . . 30

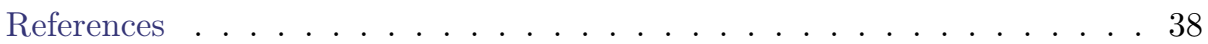

3 Nanoscale piezoelectric surface modulation for adaptive XUV and SXR optics $\quad 41$

3.1 Introduction. . . . . . . . . . . . . . . . . . . . . . 42

3.2 Concept. . . . . . . . . . . . . . . . . . . . . 43

3.3 Experimental details . . . . . . . . . . . . . . . . . . . . . . . . . . . . . . . . . . . . . . 45

3.4 Results and discussions. . . . . . . . . . . . . . . . . . . . 46

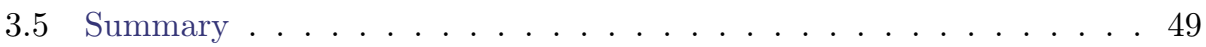

References .................... . . 50

4 Influence of template layer on structure and ferroelectric properties of $\mathrm{PbZr}_{0.52} \mathrm{Ti}_{0.48} \mathrm{O}_{3}$ films 53

4.1 Introduction. . . . . . . . . . . . . . . . . . . . 54

4.2 Experimental details . . . . . . . . . . . . . . . . . . . . . . . . . . . . . . . . 55

4.2.1 Film deposition and fabrication . . . . . . . . . . . 55

4.2.2 Analysis and characterization . . . . . . . . . . . . 56

4.3 Results and discussion . . . . . . . . . . . . . . . . 56

4.3.1 Microstructure of PZT and LNO . . . . . . . . . . . . . 56

4.3.2 Crystal structure . . . . . . . . . . . . . . . 60

4.3.3 Discussion on growth mode . . . . . . . . . . . . . 62

4.3.4 Ferroelectric properties. . . . . . . . . . . . . . 64

4.4 Conclusion ....................... . . . 67

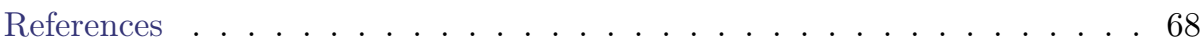


5 Hysteresis, loss and nonlinearity in epitaxial $\mathrm{PbZr}_{0.55} \mathrm{Ti}_{0.45} \mathrm{O}_{3}$ films: A polarization rotation model $\quad \mathbf{7 5}$

5.1 Introduction. . . . . . . . . . . . . . . . . . 76

5.2 Model development using experimental constraints. . . . . . . . . . . 77

5.2.1 Polarization rotation model . . . . . . . . . . . . . . 78

5.3 Experimental details . . . . . . . . . . . . . . . . . . . 84

5.4 Results and discussion . . . . . . . . . . . . . . . . . . . . . . . 85

5.4.1 Crystal structure . . . . . . . . . . . . . . . 85

5.4 .2 Measured strain and polarization response . . . . . . . . . 86

5.4 .3 Strain data . . . . . . . . . . . . . . . . . . . . . . 87

5.4 Polarization data . . . . . . . . . . . . . . . . . 91

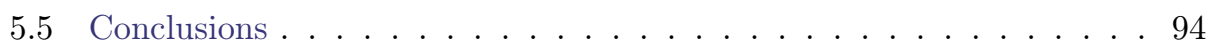

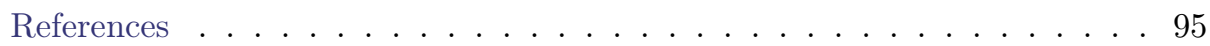

6 Hysteresis, loss and nonlinearity in epitaxial $\mathrm{PbZr}_{0.55} \mathrm{Ti}_{0.45} \mathrm{O}_{3}$ films:

$\begin{array}{ll}\text { Polarization rotation under a DC bias field } & 101\end{array}$

6.1 Introduction. . . . . . . . . . . . . . . . . . . . . 102

6.2 Polarization rotation model with applied bias. . . . . . . . . . . . . . . . . . . 103

6.2.1 Strain response . . . . . . . . . . . . . . . . 103

6.2.2 Polarization response. . . . . . . . . . . . . . 105

6.3 Experimental details . . . . . . . . . . . . . . . . . . . . . . . . . . . . . . . . . . . . . . . . . . . . . .

6.4 Results and discussion . . . . . . . . . . . . . . . . . . . . . . 109

6.4.1 Strain hysteresis measurements . . . . . . . . . . . . 109

6.4.2 Polarization hysteresis measurements . . . . . . . . . . . . 113

6.5 Conclusions . . . . . . . . . . . . . . . . . . 118

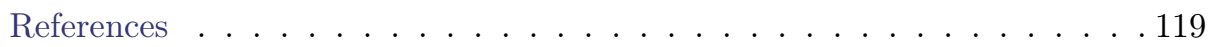

A Supporting Information Hysteresis, loss and nonlinearity in epitaxial $\mathrm{PbZr}_{0.55} \mathrm{Ti}_{0.45} \mathrm{O}_{3}$ films: A polarization rotation model $\mathbf{1 2 3}$

A.1 Rayleigh model and its extensions . . . . . . . . . . . . . . . . . . . . 124

A.2 Strain hysteresis derived from Landau-Devonshire theory . . . . . . . 126

A.3 Amplitude of the harmonics of the polarization hysteresis . . . . . . . 128

A.4 XRD analysis . . . . . . . . . . . . . . . . . . . . . . . . . . . . . . . . . . . . . . .

A.5 Small signal $d_{33}$ measurement . . . . . . . . . . . . . . . . . . . 137

A.6 Rate dependence of fit parameters. . . . . . . . . . . . . . . . . 137

A.7 Strain hysteresis loops at different frequencies . . . . . . . . . . . . . 138

A.8 Polarization hysteresis loops at different frequencies . . . . . . . . . . 138

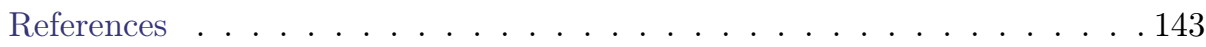

B Supporting Information Hysteresis, loss and nonlinearity in epitaxial $\mathrm{PbZr}_{0.55} \mathrm{Ti}_{0.45} \mathrm{O}_{3}$ films: Polarization rotation under a $\mathrm{DC}$ bias field $\quad 147$

B.1 Amplitude of the polarization harmonics . . . . . . . . . . . . . . . . 147

B.1.1 For $\mathbf{E}_{0}=\mathbf{1 0} \mathbf{k V ~ c m}^{-1}$ AC field amplitude . . . . . . . . . . 148

B.1.2 For $\mathbf{E}_{0}=28 \mathrm{kV} \mathrm{cm}^{-1}$ AC field amplitude . . . . . . . . . 150 
B.2 Area calculation for the polarization hysteresis loops. . . . . . . . . 153

B.3 Strain hysteresis loops at different frequencies . . . . . . . . . . . . . 154

B.4 Polarization hysteresis loops at different frequencies . . . . . . . . . . 156

B.5 Fitting of the polarization loss tangent . . . . . . . . . . . . . 159

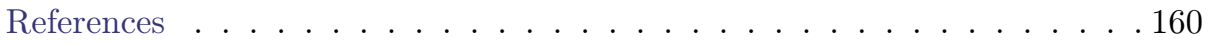

$\begin{array}{ll}\text { Summary } & 163\end{array}$

$\begin{array}{ll}\text { Samenvatting } & 165\end{array}$

$\begin{array}{ll}\text { Acknowledgments } & 167\end{array}$

$\begin{array}{lr}\text { Curriculum Vitae } & 169\end{array}$ 



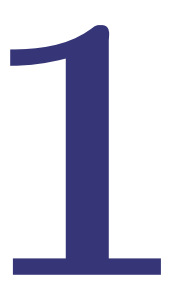

\section{Introduction}


The usage of piezoelectric materials has an impressive tradition. The direct piezoelectric effect, the generation of electrical charge upon externally applied mechanical force, was discovered by Pierre and Jacques Curie in 1880. Reversely, materials showing such behavior also generate a mechanical force upon the application of an electric field, allowing the material to change its shape and dimensions. This effect is called converse piezoelectric effect and was discovered by Gabriel Lippmann in 1881. The first usage of piezoelectrics came during World War 1, when Pierre Langevin used piezoelectrics to construct a transducer to generate sonar waves for the detection of submarines. In the following decades research was done on finding piezoelectric materials with a higher performance to allow the development of new applications. A major step was the discovery of barium titanate and lead zirconate titanite, $\mathrm{PbTi}_{1-x} \mathrm{Zr}_{x} \mathrm{O}_{3}$ (PZT) ceramics in the fifties [1].

Nowadays, piezoelectrics are found in many of our daily used technologies. Examples are piezoelectric fuel injectors, automobile collision sensors, ultrasonic cleaning bathes, piezo igniters, piezoelectric motors in microscopes, actuators for precision alignment, and ultrasounds in medicine. As technology is striving towards realizing ever smaller devices, nowadays a lot of attention is on the usage of piezoelectric thin films in MEMS applications, such as actuators [2, 3], sensors [2, 3], transducers [4], energy harvesting devices [5, 6], adaptive optics [7-9], and inkjet printer nozzles [6].

Given this broad range of applications and the state of their maturity, one could question the need for further scientific investigations. This thesis definitely does confirm the need for further research: understanding, developing and controlling piezoelectric materials is an important field of modern materials research as it enables further development, improved control and down-scaling. In the following a short overview is given on the state-of-the-art knowledge on PZT thin films used for high performance applications and the role of further research of interest.

\subsection{Motivation and outlook}

Among the most challenging piezoelectric applications is the small-scale optics at wavelengths shorter than well-explored visible and ultraviolet. More specifically the soft X-ray and extreme ultraviolet wavelength range, collectively indicated as XUV (few tenths to few tens of nanometers), is nowadays of interest in high resolution imaging, as the resolution of any imaging tool is limited by the wavelength of the light employed for imaging. For resolution in the nanometer range, the XUV range has to be used. Light sources for XUV light can be laser produced plasmas, synchrotron radiation sources or free electron lasers, e.g. PETRA III at DESY. In this wavelength range normal, refracting imaging concepts, like lenses, do not work, due to the very high absorption of almost all materials. Instead, reflective multilayer mirrors (MLM) are used [10]. Wavefront distortions and the optical figure of mirrors are in the range of the wavelength used and their manipulation requires deformation of the MLMs in the range of about half to full value of the used wavelength. As such the required precision is in the sub-nanometer to nanometer-scale. Piezoelec- 
tric thin films can address such a requirement. Other adaptive schemes fulfilling this requirement work with thermo-mechanical actuators or with thermal actuation of a heat absorbing layer under the MLM [11]. However, these schemes suffer from low spatial resolution or low actuation speed, whereas piezoelectric thin films allow high spatial resolution and fast actuation.

In this thesis, a demonstrator of an adaptive type of optic for the XUV wavelengths is presented. In this application, the piezoelectric thin film provides the possibility to adjust the surface figure of the optic, e.g. to compensate for wavefront distortions or deformation of the optical figure of the mirror. These and similar applications show that highly functional properties of the PZT thin films are required. They give direction to the research still needed.

In today's thin film piezo applications, PZT is most widely used because of its superior ferro- and piezo-electric properties. The functional properties of PZT thin films are highly dependent on materials properties such as crystal orientation, stoichiometry, and microstructure. These properties are controlled by deposition process conditions, but also by the selection of substrates or buffer layers. A higher functionality is desirable from the application point of view, as it enables new applications, or higher performance in known applications.

The $\mathrm{PbTi}_{1-x} \mathrm{Zr}_{x} \mathrm{O}_{3}$ film stoichiometry with the most favorable functional properties is found at $x=0.48$, the so-called morphotropic phase boundary composition [12-14]. The morphotropic phase boundary is the transition region between the tetragonal and rhombohedral phase of PZT. The phase of PZT is dependent on the $\mathrm{Zr}$ /Ti ratio. Films grown in (001)-orientation, the out-of-plane crystal orientation, have shown a higher functional response compared to other orientations, both theoretically $[15,16]$ and experimentally $[2,17,18]$. The growth on affordable and industrially usable substrates such as $\mathrm{Si}$, glass or metals require so-called buffer layers to achieve proper crystal orientation control [19-23]. Of particular interest are chemically synthesized, unit-cell-thick, oxide nanosheets, that allow control of the desired crystal orientation on Si and glass substrates. The use of nanosheets does not require very high deposition temperatures, which makes them more compatible with modern CMOS processes [24-27]. The film microstructure affects the ferroelectric and piezoelectric response of the film, due to grain size and domain structure dependence [28-30] and due to the influence of the microstructure on the elastic parameters of the film [31-37].

The influence of film microstructure, stoichiometry and crystal orientation on the functional properties has been studied in detail for various deposition techniques, such as chemical solution deposition (CSD) [29, 38-40], magnetron sputtering [29, 41-44] or pulsed laser deposition (PLD) [35, 36, 45-48]. In this thesis the thin films are deposited by PLD, as PLD allows very precise control of stoichiometry, orientation and hence the film quality $[49,50]$. While the dependence of the deposition conditions of the film's microstructure and functional properties are well studied, the effect of the deposition conditions of the template layer, the layer on 
which the film is grown, has not been addressed so far. An investigation on this effect is of interest as it could allow further improvement and control of the functional film properties due to the use of a new independent deposition parameter in the form of a film growth template. This thesis will show that the effect of the template layer is of major relevance. The deposition conditions of the template layer work as an independent parameter set to control the subsequently deposited film microstructure and thus the ferroelectric properties.

One known challenge for piezoelectric applications is the hysteresis and nonlinearity of the polarization and strain response of the film. The hysteresis and nonlinearity manifest themselves as e.g. positioning inaccuracies in actuators or energy loss in energy harvesting devices [51], as such they are limiting the performance of applications. The description and control of hysteresis and nonlinearity has always been an important research topic. Even though there are control models [52, 53], that allow reduction of hysteresis by using complicated control algorithms, it is of interest to identify the root causes of hysteresis. Is hysteresis related to the film quality, the amount of grain boundaries, or is it related to small defects, like dislocations or oxygen vacancies in the material? Answering this question could allow the control of hysteresis by tuning the material properties.

The viscous interaction of domains was proposed by several studies as the cause of hysteresis and the associated dielectric and piezoelectric losses for small applied fields before polarization switching happens, so-called sub-coercive fields. The models reported so far in literature describe the losses by viscous domain wall (DW) motion, but these models cannot fully explain all observed experimental loss behavior [54-58]. Damjanovic showed that the hysteresis and nonlinearity in PZT ceramics and CSD thin films can be well described by an adaption of the Rayleigh model [59, 60], which is often used to describe the hysteresis in ferromagnets [61]. The Rayleigh model describes the nonlinear field response, in which hysteresis is a consequence of stochastic interaction of DWs with defects. The interaction of the DWs with defects can be described as jumps of domain walls from one energy minimum to another, similar to Barkhausen jumps [62, 63].

As the functional properties of materials with different crystal symmetries and crystal orientations respond distinctly different to the applied electric field [64], one expects that hysteresis and the nonlinearity behave also differently for different crystallinities. However, the widely used Rayleigh model does not take crystal symmetry into account. A model that takes crystal symmetry into account could lead to a new and better understanding of the causes of hysteresis. For such a study epitaxial films are promising model systems, because of their high resemblance to perfect single crystal systems with low levels of defects. To our knowledge there is no hysteresis model that takes the crystal symmetry into account.

This thesis will introduce a new model, the so called Polarization rotation model, to describe hysteresis, loss and nonlinearity in ferroelectric epitaxial thin films. The new model describes loss and hysteresis by two separated physical pro- 
cesses, the rotation of the polarization vector in the unit cell and viscous interactions of the domains. As such hysteresis is not connected to just a stochastic process anymore, but is connected to the unit-cell deformation. The effect of polarization rotation is characteristic for monoclinic crystal symmetries and as such the model relates the description of hysteresis, loss and nonlinearity to the monoclinic crystal symmetry. Another strong point of the new model is that it in principle allows description of hysteresis, loss and nonlinearity for all ferroelectric materials with rhombohedral and monoclinic crystal symmetry, where the polarization is described by polarization rotation. This makes the model applicable to not only PZT thin films but any epitaxial ferroelectric materials with polarization rotation. The model is an important step in identifying the root causes of hysteresis. Future work on this model should be aimed to investigate and identify the viscous interactions in the domains.

Furthermore, this thesis will show that the model can be adapted to also include the application of DC bias fields. Due to the bias field the model can describe hysteresis, loss and nonlinearity for a much larger field range. As such the model can also describe use cases that could be interesting for piezoelectric applications.

The adaptive optic introduced in this thesis can produce sufficient stroke that can be of interest for wavefront correction at synchrotron radiation sources or free electron lasers. Future developments there can be improving the functional properties of the film using the template effect describe in this thesis. This effect can also be of interest in energy storage and energy harvesting applications, that work with perovskite materials that show a similar behavior as the films investigated in this thesis.

The Polarization rotation model provides a new point of view on hysteresis, nonlinearity and loss of monoclinic ferroelectric epitaxial films. It is of interest for the materials science and applications field as epitaxial films get ever increasing attention for applications, but as shown in this thesis they cannot be described by the commonly used Rayleigh model. The polarization rotation model is not limited to monoclinic PZT films, but applicable to all ferroelectric materials with polarization rotation. As such it can also describe for example relaxor ferroelectrics like $\mathrm{Pb}\left(\mathrm{Mg}_{1} / 3 \mathrm{Nb}_{2} / 3\right)_{1-x} \mathrm{Ti}_{x} \mathrm{O}_{3}(\mathrm{PMN}-\mathrm{PT})$. This work can be expanded in the future to study the loss mechanisms in different materials in comparison to PZT. In addition, this work underlines the need for further research to identify the exact nature of the assumed viscous interaction in the domains, by both experimental and theoretical work.

\subsection{Thesis outline}

The thesis is structured as followed:

Chapter 2 gives a short overview of pulsed laser deposition technique and introduces shortly the used characterization techniques such as X-ray diffraction (XRD), atomic force microscopy (AFM) and scanning electron microscopy (SEM). 
For characterizing the ferro- and piezoelectric response of the PZT films, a ferroelectric tester that is combined with a double beam laser interferometer (DBLI) is utilized. This measurement setup is described with a focus on the sub-coercive field measurements of strain and polarization.

Chapter 3 presents a small-scale optics demonstrator for an application for nanoscale piezoelectric surface modulation for adaptive XUV optics. The demonstrator is based on piezoelectric thin films and allows gradually varying surface adjustments. The chapter discusses the needed layers and their usage for such an application. It is shown that with this first, small scale demonstrator the generation of the needed surface deformation of a few nanometer is well possible. The chapter sets the scope of the required precision and film control, and explores the limits of piezo-electrical film control.

Chapter 4 shows the influence of the template layer, namely $\mathrm{LaNiO}_{3}$ (LNO), on the microstructure and the ferroelectric properties of subsequently deposited PZT film. In this work, the deposition conditions of the LNO, namely deposition pressure of $\mathrm{O}_{2}$ and thickness, were varied while keeping the deposition conditions of the PZT constant, in order to isolate the different effects. The films were analyzed in terms of microstructure crystallinity and ferroelectric properties. Increased oxygen pressure and/or thickness of the LNO template leads to an increased roughness of the template and columnar growth of the PZT film. The change from a smooth dense film to a columnar film changes the ferroelectric properties. This change is attributed to the reduced and more even lateral grain size of the columnar PZT films.

Chapter 5 investigates the hysteresis, loss and nonlinearity in epitaxial monoclinic PZT films. It is found that the commonly used Rayleigh model cannot explain the observed behavior. We show that this behavior is appropriately described by a new model, the polarization rotation model, that is based on the rotation of the polarization vector within the unit cell under an applied field, with viscous domain interactions accompanying the unit cell deformation. It is shown that the nonlinear response and the hysteretic loss originate from two separate physical processes.

Chapter 6 presents the investigation of hysteresis, loss and nonlinearity for epitaxial monoclinic PZT films under an applied DC bias field. We show that an adaptation of the polarization rotation model, that takes DC bias fields into account, describes the experimental behavior very well and can explain the reduction of loss and nonlinearity that is observed in comparison to the case of no applied bias. The applied bias field allows the description for AC fields higher than the coercive field. 


\section{References}

[1] B. Jaffe, R. S. Roth, and S. Marzullo, Piezoelectric Properties of Lead Zirconate-Lead Titanate Solid-Solution Ceramics, Journal of Applied Physics 25, 809 (1954).

[2] P. Muralt, Ferroelectric thin films for micro-sensors and actuators: a review, Journal of Micromechanics and Microengineering 10, 136 (2000).

[3] P. Muralt, R. G. Polcawich, and S. Trolier-McKinstry, Piezoelectric Thin Films for Sensors, Actuators, and Energy Harvesting, MRS Bulletin 34, 658 (2009).

[4] B. Belgacem, F. Calame, and P. Muralt, Piezoelectric micromachined ultrasonic transducers with thick PZT sol gel films, Journal of Electroceramics 19, 369 (2007).

[5] W. J. Choi, Y. Jeon, J. H. Jeong, R. Sood, and S. G. Kim, Energy harvesting MEMS device based on thin film piezoelectric cantilevers, Journal of Electroceramics $\mathbf{1 7}, 543$ (2006).

[6] C.-B. Eom and S. Trolier-McKinstry, Thin-film piezoelectric MEMS, MRS Bulletin 37, 1007 (2012).

[7] I. Kanno, T. Kunisawa, and H. Kotera, Piezoelectric Deformable MEMS Mirror for Adaptive Optics Composed of PZT Thin Films, in IEEE/LEOS International Conference on Optical MEMS and Their Applications Conference, 2006. (2006) pp. 156-157.

[8] R. H. Wilke, S. Trolier-McKinstry, P. Reid, and D. Schwartz, PZT piezoelectric films on glass for Gen-X imaging, SPIE Optical Engineering + Applications, Vol. 7803 (SPIE, 2010).

[9] M. Bayraktar, A. Chopra, G. Rijnders, K. Boller, and F. Bijkerk, Wavefront correction in the extreme ultraviolet wavelength range using piezoelectric thin films, Optics Express 22, 30623 (2014).

[10] E. Louis, A. E. Yakshin, T. Tsarfati, and F. Bijkerk, Nanometer interface and materials control for multilayer EUV-optical applications, Progress in Surface Science 86, 255 (2011).

[11] M. Bayraktar, Adaptive multilayer optics for extreme ultraviolet wavelengths, Ph.D. thesis (2015).

[12] R. Guo, L. E. Cross, S. E. Park, B. Noheda, D. E. Cox, and G. Shirane, Origin of the High Piezoelectric Response in $\mathrm{PbZr}_{1-x} \mathrm{Ti}_{x} \mathrm{O}_{3}$, Physical Review Letters 84, 5423 (2000).

[13] M. J. Haun, E. Furman, S. J. Jang, and L. E. Cross, Thermodynamic theory of the lead zirconate-titanate solid solution system, part V: Theoretical calculations, Ferroelectrics 99, 63 (1989). 
[14] B. Jaffe, W. R. Cook, and H. Jaffe, Chapter 7 - Solid Solutions of Pb(Ti, Zr, $\mathrm{Sn}, \mathrm{Hf}) \mathrm{O}_{3}$, in Piezoelectric Ceramics, edited by B. Jaffe, W. R. Cook, and H. Jaffe (Academic Press, 1971) pp. 135-183.

[15] X.-h. Du, U. Belegundu, and K. Uchino, Crystal orientation dependence of piezoelectric properties in lead zirconate titanate: Theoretical expectation for thin films, Japanese Journal of Applied Physics 36, 5580 (1997).

[16] J. Ouyang, S. Y. Yang, L. Chen, R. Ramesh, and A. L. Roytburd, Orientation dependence of the converse piezoelectric constantsfor epitaxial single domain ferroelectric films, Applied Physics Letters 85, 278 (2004).

[17] X.-h. Du, J. Zheng, U. Belegundu, and K. Uchino, Crystal orientation dependence of piezoelectric properties of lead zirconate titanate near the morphotropic phase boundary, Applied Physics Letters 72, 2421 (1998).

[18] C. J. Kim, D. S. Yoon, J. S. Lee, C. G. Choi, W. J. Lee, and K. No, Electrical characteristics of (100), (111), and randomly aligned lead zirconate titanate thin films, Journal of Applied Physics 76, 7478 (1994).

[19] D. K. Fork, D. B. Fenner, G. A. N. Connell, J. M. Phillips, and T. H. Geballe, Epitaxial yttria-stabilized zirconia on hydrogen-terminated Si by pulsed laser deposition, Applied Physics Letters 57, 1137 (1990).

[20] R. A. McKee, F. J. Walker, and M. F. Chisholm, Crystalline Oxides on Silicon: The First Five Monolayers, Physical Review Letters 81, 3014 (1998).

[21] S. J. Wang, C. K. Ong, L. P. You, and S. Y. Xu, Epitaxial growth of yittriastabilized zirconia oxide thin film on natively oxidized silicon wafer without an amorphous layer, Semiconductor Science and Technology 15, 836 (2000).

[22] M. Dekkers, M. D. Nguyen, R. Steenwelle, P. M. t. Riele, D. H. A. Blank, and G. Rijnders, Ferroelectric properties of epitaxial $\mathrm{Pb}(\mathrm{Zr}, \mathrm{Ti}) \mathrm{O}_{3}$ thin films on silicon by control of crystal orientation, Applied Physics Letters 95, 012902 (2009).

[23] D. Diaz-Fernandez, M. Spreitzer, T. Parkelj, J. Kovač, and D. Suvorov, The importance of annealing and stages coverage on the epitaxial growth of complex oxides on silicon by pulsed laser deposition, RSC Advances 7, 24709 (2017).

[24] K. Kikuta, K. Noda, S. Okumura, T. Yamaguchi, and S.-i. Hirano, Orientation control of perovskite thin films on glass substrates by the application of a seed layer prepared from oxide nanosheets, Journal of Sol-Gel Science and Technology 42, 381 (2007).

[25] M. Bayraktar, A. Chopra, F. Bijkerk, and G. Rijnders, Nanosheet controlled epitaxial growth of $\mathrm{PbZr}_{0.52} \mathrm{Ti}_{0.48} \mathrm{O}_{3}$ thin films on glass substrates, Applied Physics Letters 105, 132904 (2014). 
[26] Y. Minemura, Y. Kondoh, H. Funakubo, and H. Uchida, One-Axis-Oriented Crystal Growth of Lead Zirconate Titanate Thin Films on Metal Substrates Using Perovskite-Type Oxide Nanosheet Layer, Key Engineering Materials 582, 15 (2014).

[27] M. D. Nguyen, H. Yuan, E. P. Houwman, M. Dekkers, G. Koster, J. E. ten Elshof, and G. Rijnders, Highly Oriented Growth of Piezoelectric Thin Films on Silicon Using Two-Dimensional Nanosheets as Growth Template Layer, ACS Applied Materials \& Interfaces 8, 31120 (2016).

[28] N. Setter, D. Damjanovic, L. Eng, G. Fox, S. Gevorgian, S. Hong, A. Kingon, H. Kohlstedt, N. Y. Park, G. B. Stephenson, I. Stolitchnov, A. K. Taganstev, D. V. Taylor, T. Yamada, and S. Streiffer, Ferroelectric thin films: Review of materials, properties, and applications, Journal of Applied Physics 100, 051606 (2006).

[29] N. Izyumskaya, Y. I. Alivov, S. J. Cho, H. Morkoç, H. Lee, and Y. S. Kang, Processing, structure, properties, and applications of PZT thin films, Critical Reviews in Solid State and Materials Sciences 32, 111 (2007).

[30] E. P. Houwman, M. D. Nguyen, M. Dekkers, and G. Rijnders, Intrinsic stability of ferroelectric and piezoelectric properties of epitaxial $\mathrm{PbZr}_{0.45} \mathrm{Ti}_{0.55} \mathrm{O}_{3}$ thin films on silicon in relation to grain tilt, Science and Technology of Advanced Materials 14, 045006 (2013).

[31] C. S. Ganpule, A. Stanishevsky, S. Aggarwal, J. Melngailis, E. Williams, R. Ramesh, V. Joshi, and C. P. d. Araujo, Scaling of ferroelectric and piezoelectric properties in $\mathrm{Pt} / \mathrm{SrBi}_{2} \mathrm{Ta}_{2} \mathrm{O}_{9} / \mathrm{Pt}$ thin films, Applied Physics Letters 75, 3874 (1999).

[32] S. Bühlmann, B. Dwir, J. Baborowski, and P. Muralt, Size effect in mesoscopic epitaxial ferroelectric structures: Increase of piezoelectric response with decreasing feature size, Applied Physics Letters 80, 3195 (2002).

[33] J.-H. Li, L. Chen, V. Nagarajan, R. Ramesh, and A. L. Roytburd, Finite element modeling of piezoresponse in nanostructured ferroelectric films, Applied Physics Letters 84, 2626 (2004).

[34] A. Chopra, M. Bayraktar, M. Nijland, J. E. ten Elshof, F. Bijkerk, and G. Rijnders, Tuning of large piezoelectric response in nanosheet-buffered lead zirconate titanate films on glass substrates, Scientific Reports 7, 251 (2017).

[35] M. D. Nguyen, E. P. Houwman, M. Dekkers, and G. Rijnders, Strongly Enhanced Piezoelectric Response in Lead Zirconate Titanate Films with Vertically Aligned Columnar Grains, ACS Applied Materials \& Interfaces 9, 9849 (2017).

[36] M. D. Nguyen, E. P. Houwman, H. Yuan, B. J. Wylie-Van Eerd, M. Dekkers, G. Koster, J. E. Ten Elshof, and G. Rijnders, Controlling Piezoelectric Responses in $\mathrm{Pb}\left(\mathrm{Zr}_{0.52} \mathrm{Ti}_{0.48}\right) \mathrm{O}_{3}$ Films through Deposition Conditions and Nanosheet Buffer Layers on Glass, ACS Applied Materials \& Interfaces 9, 35947 (2017). 
[37] M. D. Nguyen, E. P. Houwman, and G. Rijnders, Large piezoelectric strain with ultra-low strain hysteresis in highly c-axis oriented $\mathrm{Pb}\left(\mathrm{Zr}_{0.52} \mathrm{Ti}_{0.48}\right) \mathrm{O}_{3}$ films with columnar growth on amorphous glass substrates, Scientific Reports 7 (2017), 10.1038/s41598-017-13425-w.

[38] F. Chu, F. Xu, J. Shepard, and S. Trolier-McKinstry, Thickness Dependence of the Electrical Properties of Sol-Gel Derived Lead Zirconate Titanate Thin Films with (111) and (100) Texture, MRS Proceedings 493, 409 (1997).

[39] X. G. Tang, H. L. W. Chan, A. L. Ding, and Q. R. Yin, Processing effects on the microstructure and ferroelectric properties of $\mathrm{Pb}(\mathrm{Zr}, \mathrm{Ti}) \mathrm{O}_{3}$ thin films prepared by sol-gel process, Surface and Coatings Technology 161, 169 (2002).

[40] Z. Chen, C. Yang, S. Wang, and B. Yang, The effects of the PbO content and seeding layers upon the microstructure and orientation of sol-gel derived PZT films, Journal of Materials Science: Materials in Electronics 17, 51 (2006).

[41] I. Kanno, H. Kotera, K. Wasa, T. Matsunaga, T. Kamada, and R. Takayama, Crystallographic characterization of epitaxial $\mathrm{Pb}(\mathrm{Zr}, \mathrm{Ti}) \mathrm{O}_{3}$ films with different $\mathrm{Zr} /$ Ti ratio grown by radio-frequency-magnetron sputtering, Journal of Applied Physics 93, 4091 (2003).

[42] H. Jacobsen, K. Prume, B. Wagner, K. Ortner, and T. Jung, High-rate sputtering of thick PZT thin films for MEMS, Journal of Electroceramics 25, 198 (2010).

[43] O. Nordseth, C. C. You, E. Folven, S. Gariglio, A. Sambri, J.-M. Triscone, J. W. Reiner, C. H. Ahn, T. Tybell, and J. K. Grepstad, Growth and characterization of $(\mathrm{Pb}, \mathrm{La})(\mathrm{Zr}, \mathrm{Ti}) \mathrm{O}_{3}$ thin film epilayers on $\mathrm{SrTiO}_{3}$-buffered Si(001), Thin Solid Films 518, 5471 (2010).

[44] S. Yoshida, H. Hanzawa, K. Wasa, M. Esashi, and S. Tanaka, Highly c-axisoriented monocrystalline $\mathrm{Pb}(\mathrm{Zr}, \mathrm{Ti}) \mathrm{O}_{3}$ thin films on $\mathrm{Si}$ wafer prepared by fast cooling immediately after sputter deposition, IEEE Transactions on Sonics and Ultrasonics 61, 1552 (2014).

[45] J. S. Horwitz, K. S. Grabowski, D. B. Chrisey, and R. E. Leuchtner, In situ deposition of epitaxial $\mathrm{PbZr}_{x} \mathrm{Ti}_{1-x} \mathrm{O}_{3}$ thin films by pulsed laser deposition, Applied Physics Letters 59, 1565 (1991).

[46] M. Tyunina and S. Leppävuori, Effects of laser fluence, size, and shape of the laser focal spot in pulsed laser deposition using a multielemental target, Journal of Applied Physics 87, 8132 (2000).

[47] T. J. Zhu, L. Lu, and M. O. Lai, Pulsed laser deposition of lead-zirconatetitanate thin films and multilayered heterostructures, Applied Physics A 81, 701 (2005).

[48] A. C. Galca, V. Stancu, M. A. Husanu, C. Dragoi, N. G. Gheorghe, L. Trupina, M. Enculescu, and E. Vasile, Substrate-target distance dependence of structural 
and optical properties in case of $\mathrm{Pb}(\mathrm{Zr}, \mathrm{Ti}) \mathrm{O}_{3}$ films obtained by pulsed laser deposition, Applied Surface Science 257, 5938 (2011).

[49] D. H. A. Blank, M. Dekkers, and G. Rijnders, Pulsed laser deposition in twente: from research tool towards industrial deposition, Journal of Physics D: Applied Physics 47, 034006 (2013).

[50] T. Venkatesan, Pulsed laser deposition-invention or discovery? Journal of Physics D: Applied Physics 47, 034001 (2013).

[51] H. Yang, F. Yan, Y. Lin, and T. Wang, Novel Strontium Titanate-Based Lead-Free Ceramics for High-Energy Storage Applications, ACS Sustainable Chemistry \& Engineering 5, 10215 (2017).

[52] L. Liu and Y. Yang, Modeling and Precision Control of Systems with Hysteresis, in Modeling and Precision Control of Systems with Hysteresis, edited by L. Liu and Y. Yang (Butterworth-Heinemann, San Diego, 2016) Book section 2-5, pp. 5-139.

[53] J. Gan and X. Zhang, A review of nonlinear hysteresis modeling and control of piezoelectric actuators, AIP Advances 9, 040702 (2019).

[54] V. Postnikov, V. Pavlov, S. Gridnev, B. Darinskii, and I. Glozman, Internal friction in $\mathrm{Pb}_{0.95} \mathrm{Sr}_{0.05} \mathrm{Zr}_{0.53} \mathrm{Ti}_{0.47} \mathrm{O}_{3}+3 \% \mathrm{PbO}$ ferroelectric ceramic, Bulletin of the Russian Academy of Sciences: Physics 31, 1888 (1967).

[55] V. Postnikov, V. Pavlov, S. Gridnev, and S. Turkov, Interaction between $90^{\circ}$ domain walls and point defects of the crystal lattice in ferroelectric ceramics, Physics of the Solid State 10, 1267 (1968).

[56] B. Laikhtman, Flexural vibrations of domain walls and dielectric dispersion of ferroelectrics, Physics of the Solid State 15, 62 (1973).

[57] J. O. Gentner, P. Gerthsen, N. A. Schmidt, and R. E. Send, Dielectric losses in ferroelectric ceramics produced by domain-wall motion, Journal of Applied Physics 49, 4485 (1978).

[58] G. Arlt and H. Dederichs, Complex elastic, dielectric and piezoelectric constants by domain wall damping in ferroelectric ceramics, Ferroelectrics 29, 47 (1980).

[59] D. Damjanovic and M. Demartin, The Rayleigh law in piezoelectric ceramics, Journal of Physics D: Applied Physics 29, 2057 (1996).

[60] D. Damjanovic, Hysteresis in Piezoelectric and Ferroelectric Materials, in The Science of Hysteresis, edited by G. Bertotti and I. D. Mayergoyz (Academic Press, Oxford, 2006) Book section 4, pp. 337-465.

[61] L. Rayleigh, XXV. Notes on electricity and magnetism.-III. On the behaviour of iron and steel under the operation of feeble magnetic forces, The London, Edinburgh, and Dublin Philosophical Magazine and Journal of Science 23, 225 (1887). 
[62] G. Bertotti, Energetic and thermodynamic aspects of hysteresis, Physical Review Letters 76, 1739 (1996).

[63] G. Bertotti, V. Basso, and G. Durin, Random free energy model for the description of hysteresis, Journal of Applied Physics 79, 5764 (1996).

[64] K. Vergeer, Structure and functional properties of epitaxial $\mathrm{PbZr}_{x} \mathrm{Ti}_{1-x} \mathrm{O}_{3}$ films, Phd thesis (2017). 


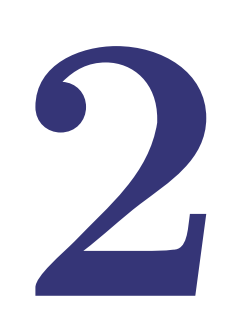

\section{Experimental}




\subsection{Thin film fabrication}

All films discussed in this thesis have been deposited by Pulsed Laser Deposition (PLD) in order to obtain stoichiometric, (001)-oriented crystal films. In PLD, the film is grown by ablation of a target material by short pulses of a focused laser. A plasma plume forms and expands, the ablated material travels from the plume and is thus deposited on the substrate, see Fig. 2.1. In PLD the deposition pressure (pressure of the ambient gas during deposition), substrate temperature and deposition rate is controlled independently, and this allows tuning of the growth conditions to obtain high quality thin films. One particular advantage of PLD is the ability of stoichiometric transfer of material from the target to the film for most of the deposition conditions. [1-3]

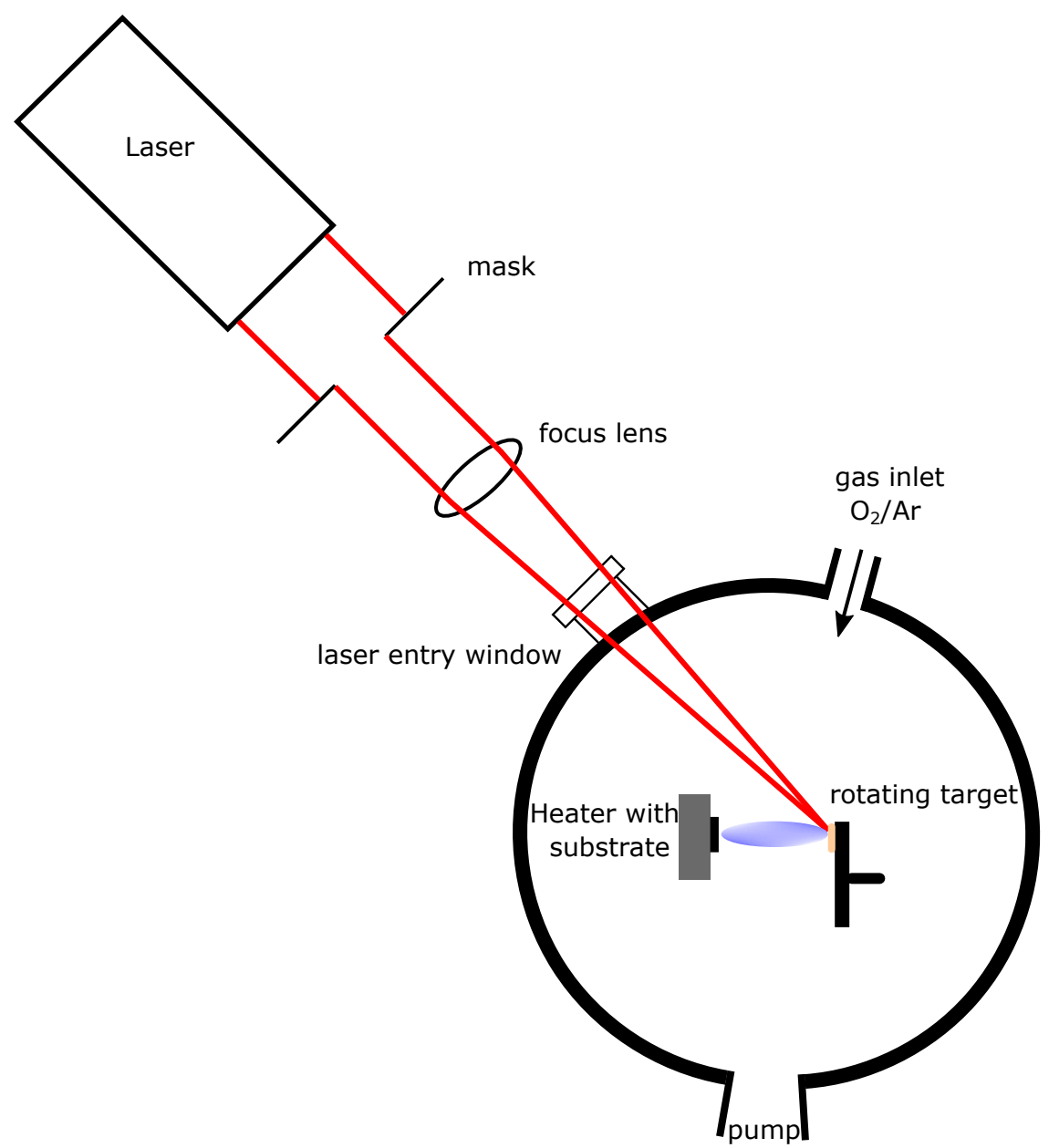

Figure 2.1: Schematic view of a PLD setup 


\subsection{Characterization}

\subsubsection{Crystal structure}

X-ray diffraction (XRD) was used to obtain crystal structure information of the thin films, such as phase and orientation. Each crystalline structure of a certain material has distinct spacing between the crystal planes ( $d$-spacing), that differs with the crystal direction. Comparison of the measured d-spacing with known theoretical $d$-spacings from a powder diffraction database allows determination of the crystal structure and identifying the crystal orientation of the film and substrate. To obtain information on the $d$-spacing of the film and the substrate, a $\omega-2 \theta$ scan, also known as $\theta-2 \theta$ scan, was used, where $2 \theta$ is the angle between the incoming beam and the detector and $\omega$ is the angle between the sample and the incoming beam, see Fig. 2.2. Specifically, in this thesis, $\omega$ is the angle between the selected crystal planes of the substrate, always the (001) plane, and the incoming beam. In the $\omega-2 \theta$ scan the $2 \theta$ angle is changed and $\omega$ is kept equal to half of $2 \theta$. Only when Bragg's condition is achieved for the X-rays reflected from the crystal planes of the film, the signal will be registered in the detector resulting in a diffraction peak. Using Bragg's law the $d$-spacing of the diffraction peak is calculated out of its $2 \theta$ position by:

$$
n \lambda=2 d \sin (\theta)
$$

with $n$ a positive integer corresponding to the diffraction order, $\lambda$ the wavelength of the X-rays, and $d$ the $d$-spacing between the crystal planes. The texture, polycrystalline nature of the film was determined with a $\omega$-scan, often also called rocking curve. In this scan the detector is kept at the $2 \theta$ position of a diffraction peak and $\omega$ is scanned over a selected range.

To obtain also information about the in-plane spacing, reciprocal space maps (RSMs) were used. A RSM is obtained by measuring high resolution $\omega$-scans for a selected range of $2 \theta$. For the XRD measurements in chapter Chapter 4 a Malvern Panalytical X'Pert diffractometer system was used. To measure the crystal properties of the films used in Chapter 5 and Chapter 6 a Bruker D8 Discover diffractometer, equipped with an area detector (EIGER2 R 500K) was used to obtain high resolution RSMs.

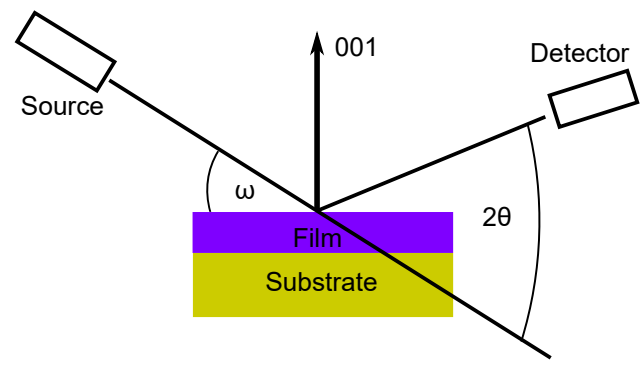

Figure 2.2: Schematic of an $\omega-2 \theta$ XRD measurement 


\subsubsection{Surface and microstructure}

For the characterization of the film microstructure, its thickness, and composition, a scanning electron microscope (SEM), a Zeiss-1550 HRSEM, has been used. In this technique a high-energy electron beam, between a few $\mathrm{keV}$ up to $50 \mathrm{keV}$, of a diameter of $1 \mathrm{~nm}$, is used to scan the surface, or cross section of the sample. The electrons from the beam, called primary electrons, exchange energy inelastically with the valence or conduction band electrons and lattice of the sample. The resulting secondary electrons have energies below $50 \mathrm{eV}$. They originate from within a few $\mathrm{nm}$ of the sample surface and are used to generate an image of the sample surface. For elemental analysis of a sample an extra detector that detects the elastically backscattered electrons was used.

The film surface was characterized by Atomic Force Microscopy (AFM) using a Bruker Icon AFM. The AFM was used in tapping mode in which the AFM tip, with a tip radius of $8 \mathrm{~nm}$, scans the film surface. The tip taps the surface close to the resonance frequency of the cantilever to have as high as possible signal amplitude. Interaction of the tip with the surface change the tip deflection, the resonance frequency and the phase of the tip. These changes are observed in the AFM by using a laser that is positioned on the backside of the cantilever. These measurements give information about local height changes on the measured sample, and were used to characterize the roughness of the films.

\subsubsection{Functional properties}

The piezoelectric and ferroelectric properties of the films, eg polarization and strain were measured with a double beam laser interferometer (aixDBLI), utilizing a ferroelectric tester (aixACCT TF-2000 Analyzer). The combination of the DBLI with a ferroelectric tester allows the measurement of the piezoelectric and ferroelectric properties simultaneously.

\section{$\operatorname{aixDBLI}$}

The DBLI measures the strain of a piezoelectric film by measuring the displacement between two illuminated surfaces of the sample due to an applied field due to the inverse piezoelectric effect. A double beam Mach-Zehnder interferometer is used for that. The advantage over single beam interferometer techniques is that any motion inside the optical path due to bending of the sample is suppressed. Hence, the bending of the sample does not contribute to the measured displacement. This allows the measurement of the pure out-of-plane displacement and as such out-of-plane piezoelectric coefficient. For this the sample needs a reflective top and bottom surface. In this thesis all films of which their functional properties were characterized, were fabricated in a parallel plate configuration with electrodes of $300 \times 300 \mathrm{\mu m}^{2}$ area, with platinum as the top layer. All samples measured with the DBLI have been deposited on double side polished substrates that reflect the laser also from the sample bottom. In the interferometer set-up one beam (measurement beam) is reflected from the top and bottom of the sample while the path length of the other beam (reference beam) is kept constant. For a change of the sample thickness, eg 
a displacement of the sample due to an applied electric field, the path length of the measurement beam is changed thus changing the intensity of the center of the interference pattern.

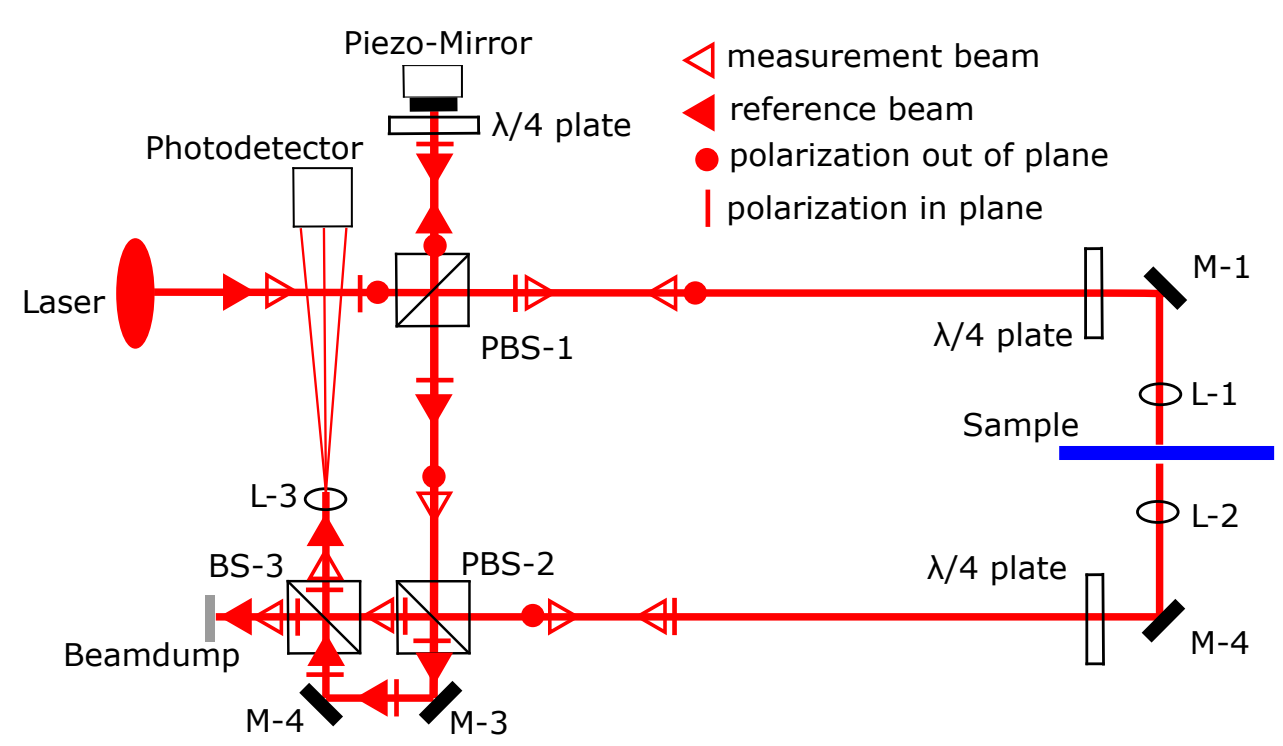

Figure 2.3: Schematic beam path of the DBLI

Fig. 2.3 shows the optical beam path of the DBLI. The laser beam generated by a He-Ne laser, enters the polarizing beam splitter (PBS-1), the in plane polarized part of the beam is transmitted and is used as the measurement beam whereas the out of plane polarized part of the beam is reflected by $90^{\circ}$ and is used as the reference beam. The measurement beam follows the top measurement arm, passes a $\lambda / 4$ plate and is reflected by the mirror M-1 and focused by lens L- 1 on the top of the sample, where it gets reflected and travels back to the beam splitter PBS-1. As it passes the $\lambda / 4$ plate twice, the polarization of the returning measurement beam is rotated by $90^{\circ}$, resulting in a downwards reflection at the beam splitter PBS-1 and another $90^{\circ}$ reflection at beam splitter PBS-2. It then travels along the bottom arm, passing another $\lambda / 4$ plate before being reflected and focused on the sample backside by mirror M-2 and lens L-2. After being reflected by the sample, it travels back passing the $\lambda / 4$ plate for the second time, rotating the beams polarization, such that the beam is transmitted in the beam splitter PBS-2. Reaching the beam splitter BS3, half of the beam is transmitted to a beam dump and the other half is reflected upwards towards the photodetector. The reference beam is reflected upwards in beam splitter PBS-1, passes the $\lambda / 4$ plate and is reflected by the piezomirror. The piezo-mirror can be shifted along the beam path, to change the length of the reference beam. The position of the piezo-mirror is calibrated such that the reference beam accounts for the thickness of the sample, controlling the operating point of the interferometer. On the way back to the beam splitter PBS-1 it passes the $\lambda / 4$ plate for the second time, rotating the polarization of the reference beam. 
The beam is then transmitted by the beam splitters PBS-1 and PBS-2. Afterwards it is reflected by the mirrors M-3 and M-4, before it passes the beam splitter BS-3. Here half of the beam is reflected to the beamdump. The other half is transmitted. As the reference beam has the same polarization state as the measurement beam at the beam splitter BS-3, the two beams interfere with each other. Lens L-3 enlarges the center of the interference pattern on the photodiode where the intensity of the interference patterns is measured.

The displacement of the film $\Delta d$ is then calculated out of the intensity of the interference patter by:

$$
\Delta d=\frac{\Delta I \lambda}{2 \pi\left(I_{\max }-I_{\min }\right)}
$$

with $\Delta I$ the change of the intensity of the interference pattern, $\lambda$ the wavelength of the laser, and $I_{\max }$ and $I_{\min }$ the maximum and minimum of the intensity of the interference pattern in the working range obtained by the system calibration before each measurement. [4]

\section{Ferroelectric tester}

Characterization of the ferroelectric response of a material is done via the measurement of the polarization response as the special characteristic of ferroelectric materials are a spontaneous polarization, a non-zero polarization at zero applied electric field, that is reversible by application of an external electric field. A direct measurement of the polarization of a material is not possible. Instead Sawyer and Tower[5] used a linear reference capacitor to observe the polarization of the material by using the fact that both the capacitor and the material have an identical amount of charge displaced due to the applied voltage, see Fig. 2.4 a). The charge is directly proportional to the voltage that is build up over the capacitor, so by plotting the voltage measured over the capacitor versus the voltage applied over the ferroelectric material, they could observe the polarization hysteresis indirectly.

Nowadays the polarization is measured with the so-called virtual ground method[6], shown in Fig. 2.4 b). The virtual ground method still uses the principle of charge conservation, but measures the current that is extracted from the voltage drop over a changeable feedback resistor over an inverting operational amplifier. This method has the advantage that the full excitation voltage drops over the ferroelectric and more important the parasitic cable capacitances are electrically ineffective. This is of importance for the measurement of small ferroelectric capacitors, especially for the measurement of thin film ferroelectrics as in this thesis. The ferroelectric analyzer used in this thesis, utilizes the virtual ground method.

The polarization $P$ is calculated using the measured current by keeping in mind that polarization is the material related part of the electrical displacement field $D$. The electrical displacement field also includes the vacuum contribution $D_{0}$. It is equivalent to the charge $Q$ per area $A$ such that:

$$
D=D_{0}+P=\epsilon_{0} E+P=\frac{Q}{A}=\frac{\int I(t) d t}{A}
$$


The polarization is calculated by subtracting the vacuum contribution $D_{0}$ from the integrated current density. However, for the used electric fields for the measurement of thin films, which are in the range of $200 \mathrm{kV} \mathrm{cm}^{-1}, D_{0}$ is very small and the relative difference between $D$ and $P$ is less then $1 \%$ such that $P \approx D$.

a)

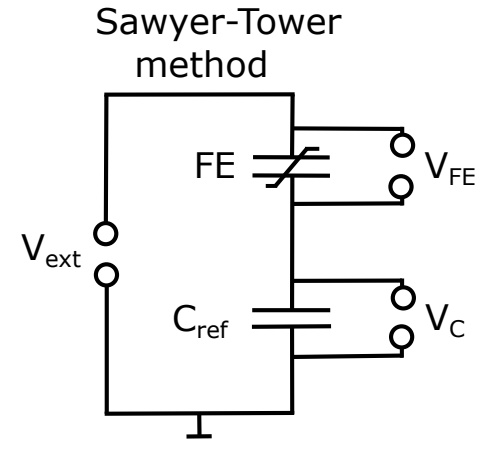

b)

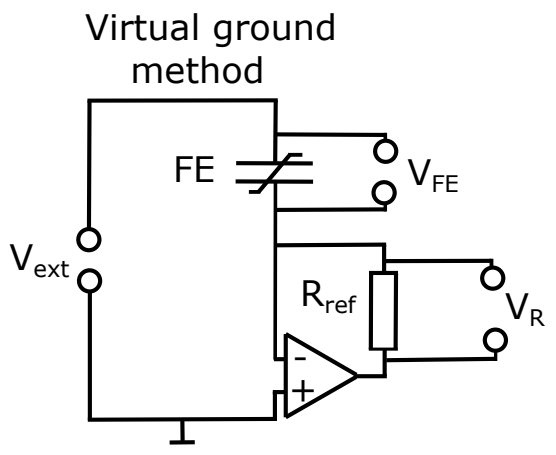

Figure 2.4: Common setups for ferroelectric hysteresis measurements: a) Sawyer-Tower and b) virtual ground method. With $V_{\text {ext }}$ the external excitation voltage, FE the ferroelectric sample, $V_{F E}$ the voltage over the ferroelectric and $V_{C} / V_{R}$ the voltage measured over the reference capacitor/resistor $\left(C_{\text {ref }} / R_{\text {ref }}\right)$.

Fig. 2.5 shows a typical measured ferroelectric hysteresis loop. Typically, the applied field is of a triangular-shape, see Fig. 2.5 a), with a field amplitude of $200 \mathrm{kV} \mathrm{cm}^{-1}$, which is high enough to observe polarization switching in practice. To ensure knowledge of the polarization direction of the sample before the triangular measurement pulse a so-called pre-pol pulse, an electric pulse with known direction and the amplitude of the measurement pulse, is applied. Typical is a negative pre-pol pulse, so that the sample starts with a negative polarization state.

The resulting current measurement, known as an I-E loop, for the applied triangular field is shown in Fig. 2.5 b). The increasing positive electric field results in a constant positive dielectric current with a strong but localized current peak at a certain field value, the switching peak. For the decreasing positive field one measures a constant negative current. For the negative applied field one observes the same behavior with opposing signs for the measured current, resulting in a negative current with localized negative peak for increasing negative field and a constant positive current for decreasing negative field. The strong and localized switching peaks are the result of the polarization switching. The narrower the peak, the higher the quality of the sample, for a perfect ferroelectric sample the switching peak is a Dirac Peak. The field value where the switching peak has its maximum is called coercive field $\left(E_{c}\right)$. This field value is the applied field needed to switch the polarization. The field range below the coercive field is known as sub-coercive field.

The value of the constant current is proportional to the leakage current of the sample. For all the measured samples in this thesis, the leakage current was orders of magnitude smaller than the measured switching current (as observed here), showing the good quality of the sample. 
Fig. 2.5 c) shows a typical polarization hysteresis, P-E loop. The P-E loop is of square shape, showing the good quality of the sample. At zero field the calculated polarization using Eq. 2.3 is negative, due to the negative pre-pol pulse, this polarization is called remnant polarization $P_{r}$. It is the amount of polarization that the sample has in its poled state. Increasing the field leads to switching of the polarization. The switching happens at the coercive field $E_{c}$, which is positioned where the polarization crosses the x-axis. As the switching happens around a very small field around $E_{c}$, the flanks of the P-E loop are very steep. An ideal P-E loop would have infinitely steep flanks and be of perfect square shape. More information on the relation of the shape of the P-E and I-E loop to the sample quality and properties can be found in literature. [7]

a)
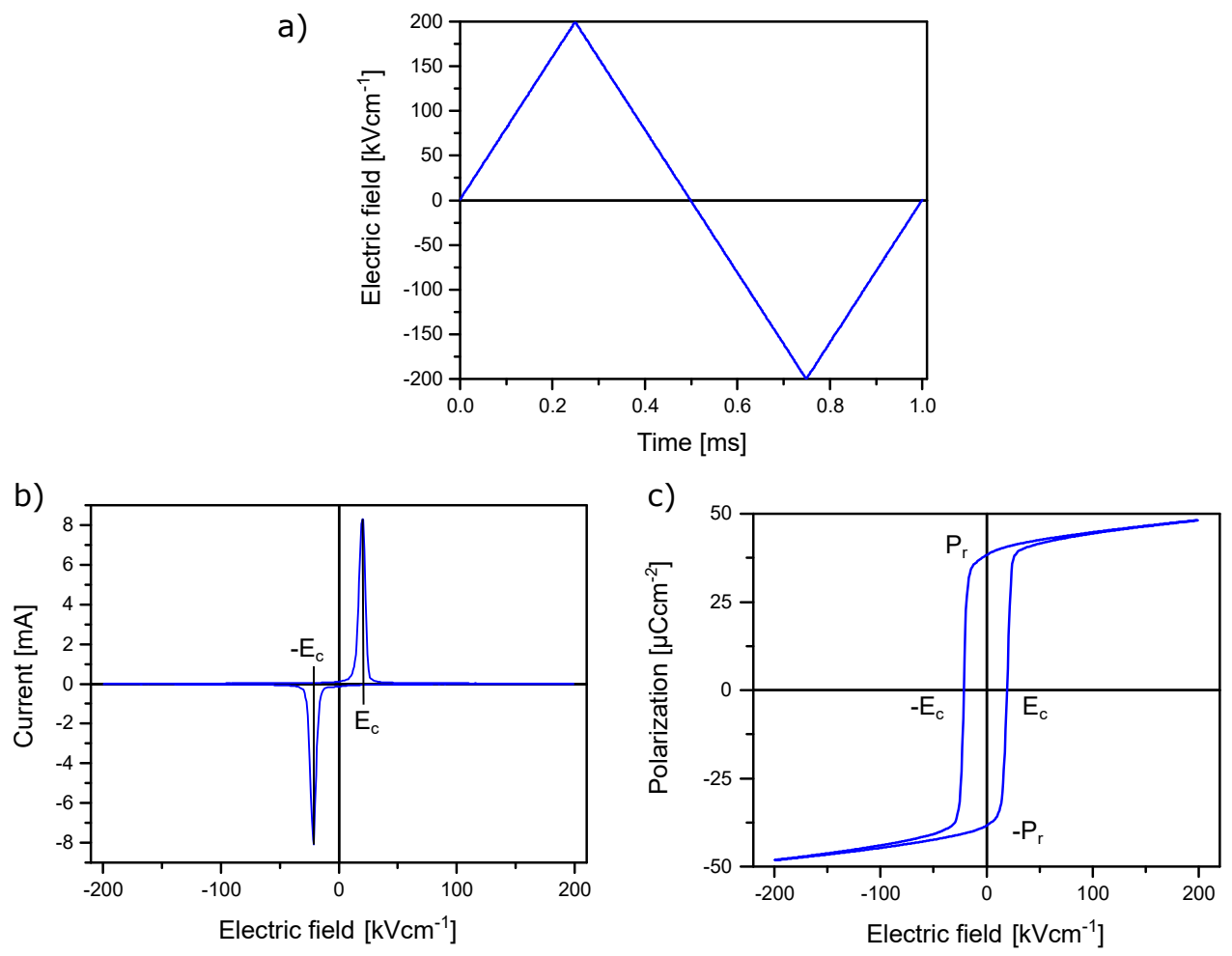

Figure 2.5: Ferroelectric hysteresis measurements: a) applied field versus time, b) measured current versus applied field (I-E loop) and c) calculated polarization versus applied field (P-E loop).

\section{Sub-coercive field hysteresis measurements}

The investigation of the hysteresis, loss and nonlinearity for epitaxial films in the sub-coercive field range described in Chapter 5 and Chapter 6 couldn't be performed with the standard measurements of the ferroelectric tester and the DBLI. The investigation required the electric field to be of sinusoidal form and with the 
ability to change field amplitude and frequency. The applied fields for the investigation were small, below $10 \mathrm{kV} \mathrm{cm}^{-1}$, such that the resulting changes of strain (around $10^{-4}$ ) and polarization (below $10 \mu \mathrm{C} \mathrm{cm}^{-2}$ ) that have been measured were very small. To increase the measurement precision the measurement data needed to be averaged. However the standard measurements of the aixACCT DBLI and ferroelectric tester, only allow averaging when a pre-pol pulse is used before the measurement. The amplitude of the pre-pol pulse of the aixACCT system cannot be changed and is automatically fixed to the amplitude of the field. In case of the sub-coercive field measurements this leads to different starting conditions of the samples due to the pre-pol pulse for each measurement having a different amplitude. In addition, the standard measurement for aixACCT systems only gives access to the averaged measured data and not to the raw data of the single measurements, which is of interest to have an estimate of the measurement precision.

To accommodate all of these requirements, the strain and polarization hysteresis loops for the sub-coercive field were measured by applying a so-called manual waveform. A manual waveform is generated by the user using the manual wavefront generator of the software of the ferroelectric tester. For the measurements all wavefronts consisted of 20 sine waves after each other with a set frequency and amplitude, to have a sufficient amount of datapoints to average. Each sine wave consisted of 128 measurement points.

Fig. 2.6 a) shows the applied electric field $E$ of such a manual waveform for a frequency and amplitude of the sine of $2 \mathrm{kHz}$ and $8 \mathrm{kV} \mathrm{cm}^{-1}$. To ensure that the applied field consist of only a sine with the selected measurement frequency, the measured data was investigated with Fourier transformation.

A Fourier transformation decomposes a signal measured in time into its constituent frequencies, transforming it from its representation in time domain into its representation in frequency domain. The Fourier transform of a function $a(a=$ $A \sin (2 \pi f t))$ is given by $\hat{a}$ as:

$$
\hat{a}(f)=\int_{-\infty}^{\infty} a(t) e^{-2 \pi i t f} d t,
$$

and the signal can be transformed back from the frequency into the time domain by the inverse Fourier transform:

$$
a(t)=\int_{-\infty}^{\infty} \hat{a}(f) e^{2 \pi i t f} d f .
$$

For repeating signals such as sinusoidal waves the observation of harmonics of the applied signal is a known phenomenon. Harmonics of a wave signal are defined as a wave with a frequency that is a positive integer multiple of the frequency of the original wave. The frequency of the original wave is called fundamental harmonic. Fig. 2.6 b) shows the Fourier spectrum of the applied field, using the Fast Fourier Transform (FFT) algorithm of Matlab, based on Eq. 2.4 on the measured field in time domain. The Fourier spectrum shows that the applied field only consist a high intensity signal peak at the fundamental harmonic at $2 \mathrm{kHz}$ plus low intensity 
noise at all other frequencies. No higher order harmonics are observed, such that any observed higher order signal for the measured strain or polarization does not originate from the applied field. The input signal-to-noise ratio (SNR) is about $10^{3}$.
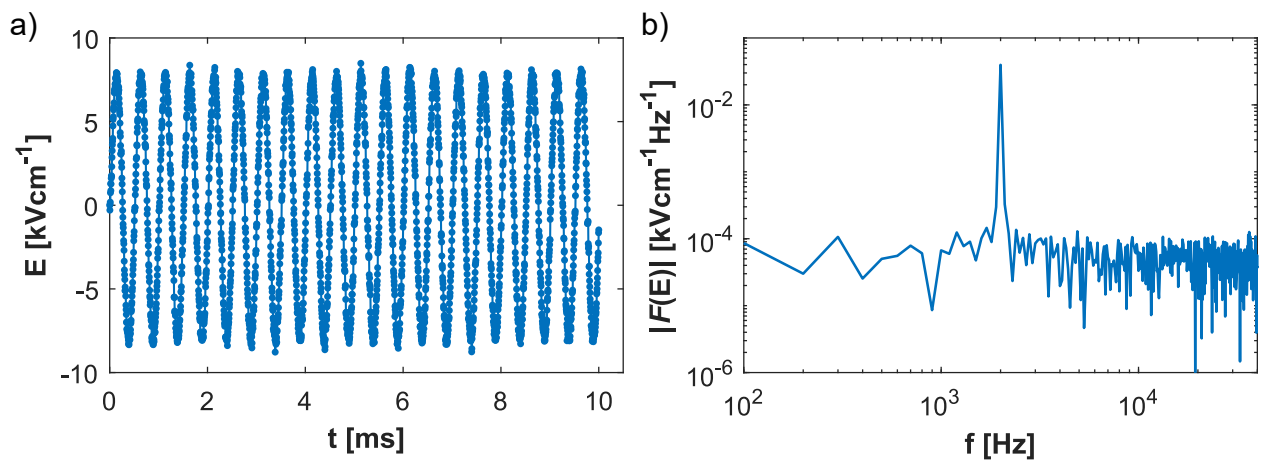

Figure 2.6: Applied field $E$ in a) time domain and b) in frequency domain.

Data analysis for sub-coercive field hystereis measurements

Fig. 2.7 shows in the left and right columns subfigures of the measured strain $\mathrm{S}$ and measured polarization for the applied field of $8 \mathrm{kV} \mathrm{cm}^{-1}$ and $2 \mathrm{kHz}$, generated by the manual waveform shown in Fig. 2.6. The first row of the subfigures shows the measured signal in time domain, the second row shows the measured signal plotted with respect to the electric field, the most common representation of the measured strain and polarization, and the last row shows the measured signal in frequency domain. The raw strain data in Fig. 2.7 a) is noisy and subject to drift in the time domain, for clarity only every second measurement point is plotted. As a result of this noise and drift in time domain, the plot of the raw strain with respect to field Fig. $2.7 \mathrm{c}$ ) is very noisy and no hysteretic loop with a clear opening can be observed. In the frequency domain, Fig. $2.7 \mathrm{e}$ ), the raw strain only shows the fundamental frequency and no higher harmonics can be identified apart from random noise peaks in the white noise. These random noise peaks originate from numerical artifact of the FFT algorithm, as they have a very small peak width. Below the fundamental frequency there is frequency dependent noise, which is explained by the drift in time domain.

The raw strain measurement data is too noisy to be used for further analysis without further data treatment. As the frequency spectrum only shows a strong signal at the fundamental frequency, a bandpass filter is applied around the fundamental frequency to filter the data. The width of the bandpass filter is selected such, that only the peak passes the filter and no frequency components that are not part of the natural broadening of the fundamental harmonic, see the orange line in Figure Fig. 2.7e). All frequency components outside of the orange line are set to zero and thus eliminated. The data is then transformed back to the time domain using the Inverse Fast Fourier Transform (IFFT) algorithm of Matlab based on Eq. 2.5, shown in Fig. 2.7 a) in orange. The such filtered data in time domain 
shows a sinusoidal shape with constant amplitude and no drift. The filtered strain plotted with respect to field shows an open ellipsoidal hysteresis loop, see Fig. $2.7 \mathrm{c}$ ) orange dots (for visibility only every 4th data point is plotted). The filtered data is then averaged over the 20 measured cycles to increase the SNR.

The raw polarization data in time domain is shown in Fig. $2.7 \mathrm{~b}$ ). The measured polarization has a constant amplitude, but its level drifts in time. This drift is also visible in Fig. $2.7 \mathrm{~d}$ ), where the polarization is plotted with respect to the electric field. The hysteresis loop is drifting on the y-axis from one measurement cycle to another. In the frequency spectrum, Fig. $2.7 \mathrm{f}$ ), not only the fundamental harmonic is visible, but also higher harmonics up to the 5 th order are visible, that are superimposed on a decreasing frequency dependent noise baseline. As for the strain, before a further analysis of the data is possible, the polarization data needs to be filtered. For the polarization all the data except for the harmonical peaks are filtered. The orange line in Fig. $2.7 \mathrm{f}$ ) shows which data is allowed to pass the filter, all other frequencies are filtered out, eliminating the noise contributions. In this way the effect of the frequency dependent noise baseline is minimized, as the observed harmonics have a higher amplitude compared to the noise baseline. The used bandpass filter is a summation of bandpass filter around each of the harmonics form $1^{\text {st }}$ to $6^{\text {th }}$ order. The maximum order of the harmonical peaks that are allowed to pass the filter is selected due to the experimental data and the predictions of theory from Chapter 5. The resulting filtered data in the time domain and plotted with respect to the field are shown in Fig. 2.7 b) and d) in orange. In time domain the polarization is not subjected to drift of the level anymore. This is also visible in the plot with respect to field Fig. $2.7 \mathrm{~d}$ ), as all filtered data points of the 20 measurement cycles are clustered at their respective fields. To increase the SNR the filtered data is averaged over the 20 measurement cycles. 


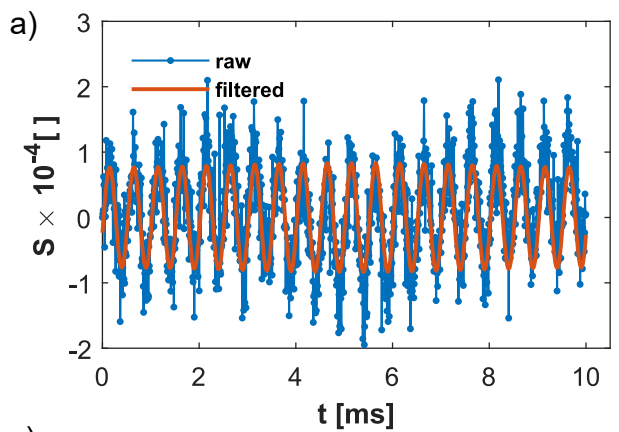

c)
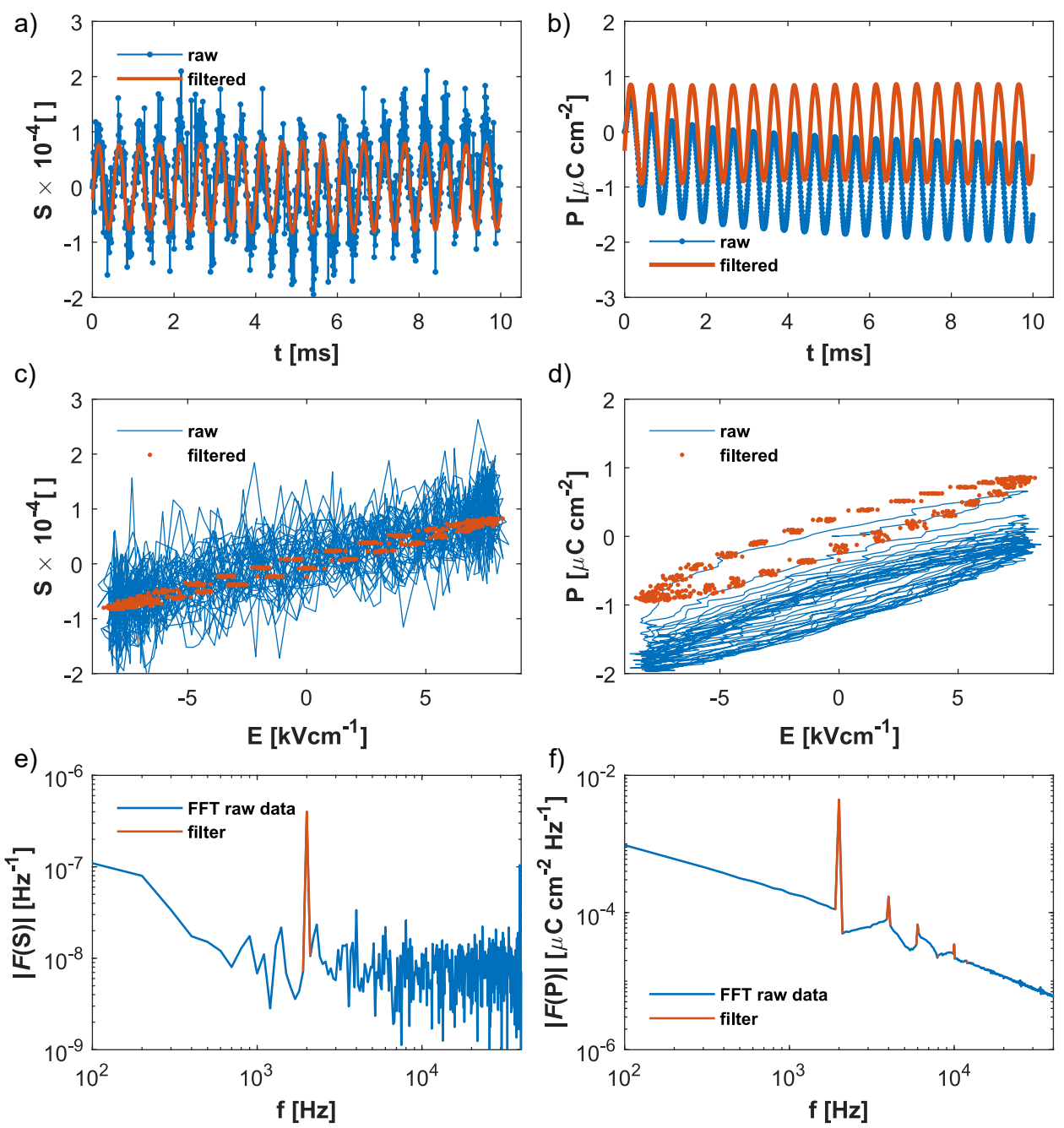

Figure 2.7: a, b) Measured strain and polarization in time domain and c, d) plotted with respect to the applied field, with blue lines representing the raw data and orange lines/dots the filtered data, e, f) representation in frequency domain for an applied field of $8 \mathrm{kV} \mathrm{cm}^{-1}$ and a frequency of $2 \mathrm{kHz}$.

\section{References}

[1] R. Eason, Pulsed Laser Deposition of Complex Materials: Progress Toward Applications, Pulsed Laser Deposition of Thin Films (Wiley, 2006).

[2] T. Venkatesan, Pulsed laser deposition-invention or discovery? Journal of Physics D: Applied Physics 47, 034001 (2013).

[3] D. H. A. Blank, M. Dekkers, and G. Rijnders, Pulsed laser deposition in Twente: 
from research tool towards industrial deposition, Journal of Physics D: Applied Physics 47, 034006 (2013).

[4] aixAACT, aixDBLI Manual, .

[5] C. B. Sawyer and C. H. Tower, Rochelle Salt as a Dielectric, Physical Review 35, 269 (1930).

[6] S. Tiedke and T. Schmitz, Electrical Characterization of Nanoscale Ferroelectric Structures, in Nanoscale Characterisation of Ferroelectric Materials: Scanning Probe Microscopy Approach, edited by M. Alexe and A. Gruverman (Springer Berlin Heidelberg, Berlin, Heidelberg, 2004) pp. 87-114.

[7] T. Schenk, E. Yurchuk, S. Mueller, U. Schroeder, S. Starschich, U. Böttger, and T. Mikolajick, About the deformation of ferroelectric hystereses, Applied Physics Reviews 1 (2014), 10.1063/1.4902396. 



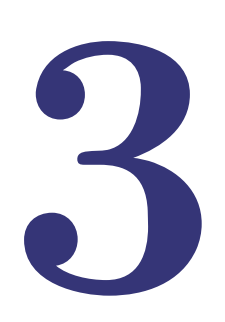

\section{Nanoscale piezoelectric surface modulation for adaptive XUV and SXR optics}

Extreme ultraviolet and soft X-ray wavelengths have ever-increasing applications in photolithography, imaging, and spectroscopy. Adaptive schemes for wavefront correction at such a short wavelength range have recently gained much attention. In this letter, we report the first demonstration of a functional actuator based on piezoelectric thin films. We introduce a new approach that allows producing a gradually varying surface deformation. White light interferometery is used to show the level of control in generating arbitrary surface profiles at the nanoscale. 


\subsection{Introduction}

Extreme ultraviolet (XUV) and soft X-ray (SXR) wavelengths are used for a wide range of applications in microscopy [1], spectroscopy [2], space research [3], and photolithography [4]. For this very short wavelengths range (1 to $40 \mathrm{~nm}$ [5]) reflective multilayer mirrors (MLM) are key optical elements [6, 7]. An MLM consists of a periodic layer structure, which enables high reflectance by constructive interference of the reflection from different interfaces based on the Bragg law. For instance, the reflectance values are in the range of $70 \%$ for the best MLMs in the $13.5 \mathrm{~nm}$ wavelength range [6]. The remaining radiation is inevitably absorbed in the MLM resulting in heat load and consequently leading to surface temperature gradients and non-uniform surface displacements. These non-uniform and time dependent surface displacements can cause wavefront aberrations on the reflected light, limiting the imaging resolution. Therefore, adaptive MLMs are needed to compensate the aberrations in order to reach the theoretical diffraction limited resolution in the optical systems.

There are two types of adaptive MLM schemes reported in the literature. The first type has the actuator at the back side of the substrate. Examples for this actuator type are thermo-mechanical actuators glued to the rear of the substrate [8], and piezoelectric film coated on the back side of thin substrates [9]. In these approaches the entire substrate deforms. This imposes a limit on the thickness of the substrate and the spatial resolution of the surface deformation. In some applications like photolithography, thick substrates are needed in order to provide a high mechanical stability. Hence, Saathof et al. [10] proposed adaptive MLMs based on thermal actuation of a heat absorbing layer coated under the MLM using a spatially extended heat source. However, this scheme and other thermal actuation methods [11] offer low actuation speed.

An approach based on piezoelectric films on the mirror side provides fast and direct actuation of the mirror surface. It also allows surface deformation with very high spatial resolution, and utilizing thick substrates is viable in this approach. The very short wavelength of operation necessitates high degree of control in the adaptive MLMs, which can be achieved by piezoelectric actuators. We have previously introduced an adaptive MLM based on piezoelectric films [12]. In that work, we demonstrated growth of piezoelectric films with sufficiently high piezoelectric coefficient and electrical breakdown strength, enabling their usage in the adaptive MLMs. Yet, the resulted surface deformation was step-like and discrete. Such steplike surface figure requires large number of electrodes to correct for aberrations that are typically smoothly varying, and it limits the wavefront correction quality.

In this letter, we present the first demonstration of a piezoelectric based functional actuator intended for wavefront correction at XUV and SXR wavelengths. First, we show how the actuator is capable of producing gradually varying surface deformation evidenced by the measurements with white light interferometry (WLI). Then, we demonstrate the steering of arbitrary surface profiles, and we discuss the 


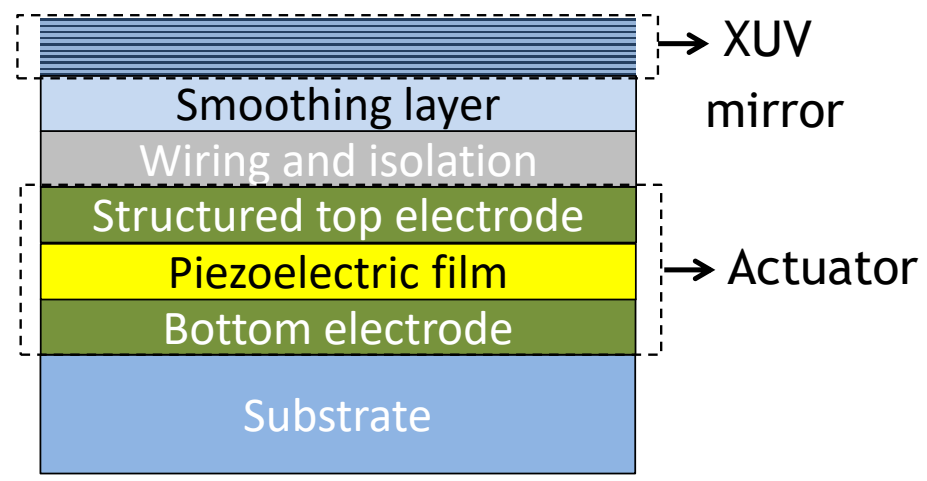

Figure 3.1: Schematic cross-section of an adaptive multilayer mirror based on a piezoelectric actuator on the mirror side; the layers and their functions are explained in the text.

future potential of the piezoelectric based adaptive MLMs.

\subsection{Concept}

The adaptive MLM is consisted of several films deposited on a substrate, and the basic layer structure is shown in Fig. 3.1. Two main components of the adaptive MLM are the piezoelectric actuator for surface deformation, and the MLM for high reflection at XUV and SXR wavelength. The piezoelectric film is sandwiched between two electrodes. The top electrode is structured into a number of pixels to allow local control of the surface displacement. Additional layers of wiring and isolation are needed to individually power each pixel. The deposition of the MLM requires a sub-nanometer smooth surface. This requirement can be addressed by deposition and polishing of a layer called "smoothing" layer prior to the MLM deposition.

By structuring the top electrode into discrete pixels as shown in Fig. 3.2(a), the applied voltage and consequently the surface displacement can be controlled independently for each electrode/pixel. This is described as the "structured top electrode" in Fig. 3.1. The discrete structuring results in abrupt changes of the applied voltage (and displacement) as shown in Fig. 3.2(c). The dashed line represents the applied voltage to the middle pixel in Fig. 3.2(a). As a result, the displacement is zero over the surface, except for that pixel.

Now, we explain how a gradually varying displacement can be produced by introducing a resistive layer between the pixels. We call this layer "mediation layer" and show it on the PZT film with green color in Fig. 3.2(b). When a voltage $(V)$ is applied to the middle pixel and the neighbouring pixels are grounded, a current passes through the mediation layer, and the voltage on the points between the middle and the neighboring pixels varies continuously. Consequently, the corresponding 


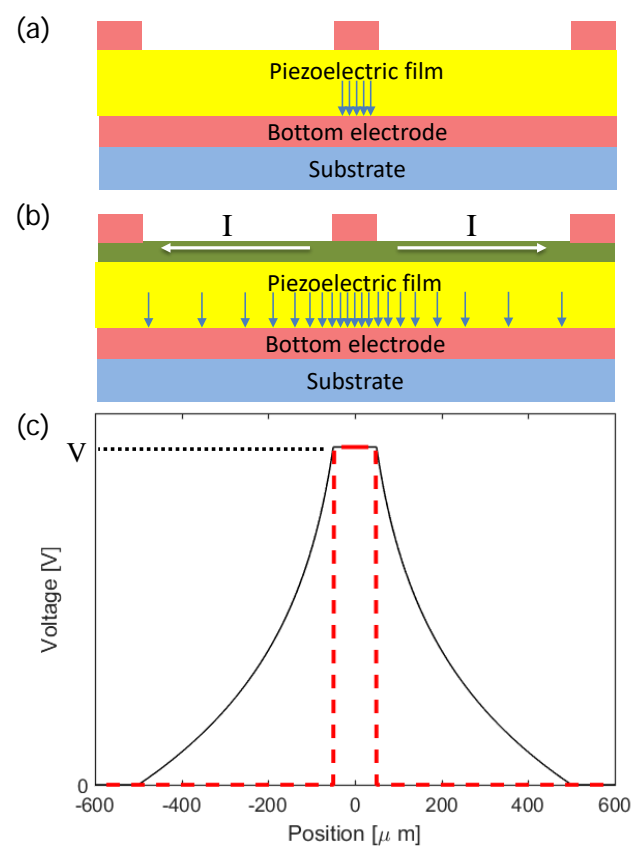

Figure 3.2: (a) Schematic drawing of a piezoelectric actuator with continuous bottom electrode and discrete top electrodes/pixels (in red); for simplicity, only three pixels are shown. The density of arrows represent the strength of the electric field when voltage $V$ is applied to the pixel in the center. (b) Schematic drawing of a piezoelectric actuator with a resistive "mediation layer" deposited on the PZT film (in green). When voltage $V$ is applied to the middle pixel, current $I$ flows through the mediation layer, causing a gradually changing electric field strength in the piezoelectric film. (c) Schematic representation of the voltage distribution in (a) and (b) shown by the dashed and solid lines, respectively.

electric field strength in the piezoelectric film and the resulting displacement varies gradually. The voltage distribution between the pixels can be calculated as follows. In a realistic scenario, each pixel is surrounded by six pixels in a hexagonal grid as shown in Fig. 3.3(b). We approximate the surrounding pixels as a continuous ring around the middle pixel. In this concentric geometry, where the middle pixel is at voltage $V$, and the surrounding pixels are at $0 \mathrm{~V}$, the voltage from the middle pixel to the outer ring decreases logarithmically as shown with the solid line in Fig. 3.2(c). The amount of vertical displacement at each point of the mediation layer depends on the local voltage and the piezoelectric coefficient in the direction of the applied electric field, called $d_{33}$, which is a function of the applied field.

A desired surface profile can be generated by correctly choosing the shape and the distance of the pixels. Note that the displacement does not depend on the mediation layer sheet resistance assuming a uniform sheet resistance, and no leakage current via the mediation layer and the piezoelectric layer underneath. However, the mediation layer sheet resistance needs to be optimized to the specific application requirements. In this work, we only qualitatively discuss the effect of a mediation 
layer. Too low sheet resistance can result in a high current within the mediation layer, and therefore, it can cause high parasitic heat loss. On the other hand, too high sheet resistance can result in a low response time of the device. With the resistor-capacitor $(\mathrm{RC})$ circuits in the piezoelectric actuator design, the time constant $\tau=\mathrm{RC}$ is higher for high mediation layer sheet resistance.

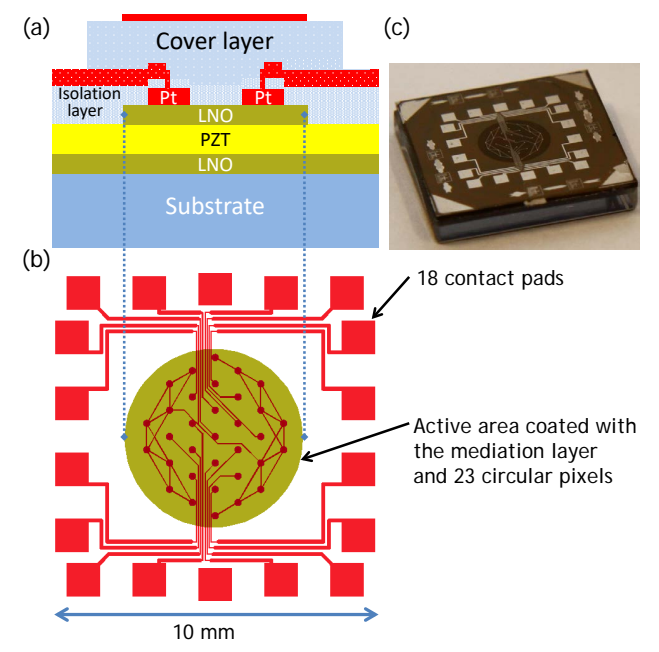

Figure 3.3: (a) Schematic layer structure, (b) top-view, and (c) photo of the piezoelectric actuator. The cover layer and the top Pt film are only depicted in the layer structure.

\subsection{Experimental details}

To demonstrate the capability and surface displacement of the piezoelectric actuator for XUV and SXR wavefront correction, we fabricated devices with the layer structure presented in Fig. 3.3(a). The electrodes $\left(\mathrm{LaNiO}_{3}, \mathrm{LNO}\right)$ and the piezoelectric layer $\left(\mathrm{PbZr}_{x} \mathrm{Ti}_{1-x} \mathrm{O}_{3}, \mathrm{PZT}\right)$ were grown by pulsed laser deposition (PLD) using a KrF excimer laser (Lambda Physik, $248 \mathrm{~nm}, 20 \mathrm{~ns}$ ) on platinized Si substrate $\left(\mathrm{Pt} / \mathrm{Ti} / \mathrm{SiO}_{2} / \mathrm{Si}\right)$. A $4 \mu \mathrm{m} \mathrm{PZT}$ film is used for this study. The growth of the LNO top electrode was optimized in order to reach the sheet resistance values of $500-2500 \Omega / \square$ needed for the mediation layer in this work. The resistivity of the LNO film was tuned by varying the growth parameters such as the background oxygen pressure and the growth temperature [13]. Hence, the top LNO layer is functioning both as the top electrode and as the mediation layer. Then, twentythree equidistant $\mathrm{Pt}$ pixels were deposited on the mediation layer by DC magnetron sputtering, and structured using photolithography and lift-off technique. The pixels have a diameter of $80 \mu \mathrm{m}$ and patterned in a hexagonal grid with a $800 \mu \mathrm{m}$ distance between pixels.

Next, We patterned the mediation layer to a region with a diameter of $5.4 \mathrm{~mm}$ at the center of the sample as shown in Fig. 3.3(b). In order to deposit the wires, 
first, a $600 \mathrm{~nm} \mathrm{SiO}_{2}$ isolation layer was deposited over the entire area of the sample by Plasma Enhanced Chemical Vapor Deposition (PECVD). The top of the pixels were etched, followed by the deposition of the wires and the contact pads based on the schematic layout shown in Fig. 3.3(b). The photo in Fig. 3.3(c) shows the device after the deposition of wires and contact pads. Note that the peripheral pixels are interconnected to provide the same boundary condition for the 15 inner pixels.

We measured the surface profiles by WLI. To prepare the samples for the measurements, an isolation layer called "cover layer" was deposited on the wires. Then, a thin Pt film was deposited on the cover layer to improve the signal to noise ratio and the optical contrast. Note that the the cover layer is not a smoothing layer as depicted in Fig. 3.1, but it is only needed for the WLI measurement. The contact pads were then connected to a multichannel power supply, i.e. the power supply had independent output channels to each of the pixels. Two contact pads that are not wired to the top pixels were connected to the bottom electrode. The WLI image measured at zero volts is subtracted from the WLI image produced when the desired power is applied to the pixels.

\subsection{Results and discussions}

First, we examine the case where all the pixels except one are at $0 \mathrm{~V}$. Fig. 3.4(a) shows the surface profile around the pixel that is powered with values in the range $0 \mathrm{~V}$ to $30 \mathrm{~V}$. In the center of the pixel where Pt is directly deposited on LNO, the applied voltage is constant over the area of the pixel; thus, the pixel displacement is nearly constant on the pixel at a value of $7 \mathrm{~nm}$ for $30 \mathrm{~V}$. In the surrounding of the pixel, we observe a gradually decreasing displacement that eventually becomes zero at the next nearest pixels with zero potential. This behavior is expected because of the mediation layer effect. The same displacement of $7 \mathrm{~nm}$ was also measured for a different sample without the thick isolation and wiring overlays. Therefore, we can expect no drop in the displacement for an adaptive mirror device containing the MLM overlay.

Fig. 3.4(b) shows the voltage dependency of the average displacement of the top of the powered pixel. Note the small non-linearity in the displacement, which could be due to electrostriction and contribution of non- $180^{\circ}$ domains as explained in[14]. A linear fit to the data results in a $d_{33}$ of $219 \mathrm{pm} \mathrm{V}^{-1}$ for the sample, which is sufficiently high for this work. However, it has been demonstrated that the $d_{33}$ can be enhanced significantly by using nanosheet buffer layer instead of platinized Si substrate $[15,16]$. It is also known that by controlling the void fraction in the piezoelectric film the effective $d_{33}$ can be enhanced significantly[17].

Next, we examine the generation of different surface profiles by applying various voltages to different pixels. Fig. 3.5(a-d) shows four different surface profiles as examples of what is possible with the piezoelectric actuator. The color scale represents values from $-2 \mathrm{~nm}$ to $6 \mathrm{~nm}$. In all the surface profiles, surface displacement is constrained in the mediation layer region with a diameter of $5.4 \mathrm{~mm}$ as expected. 

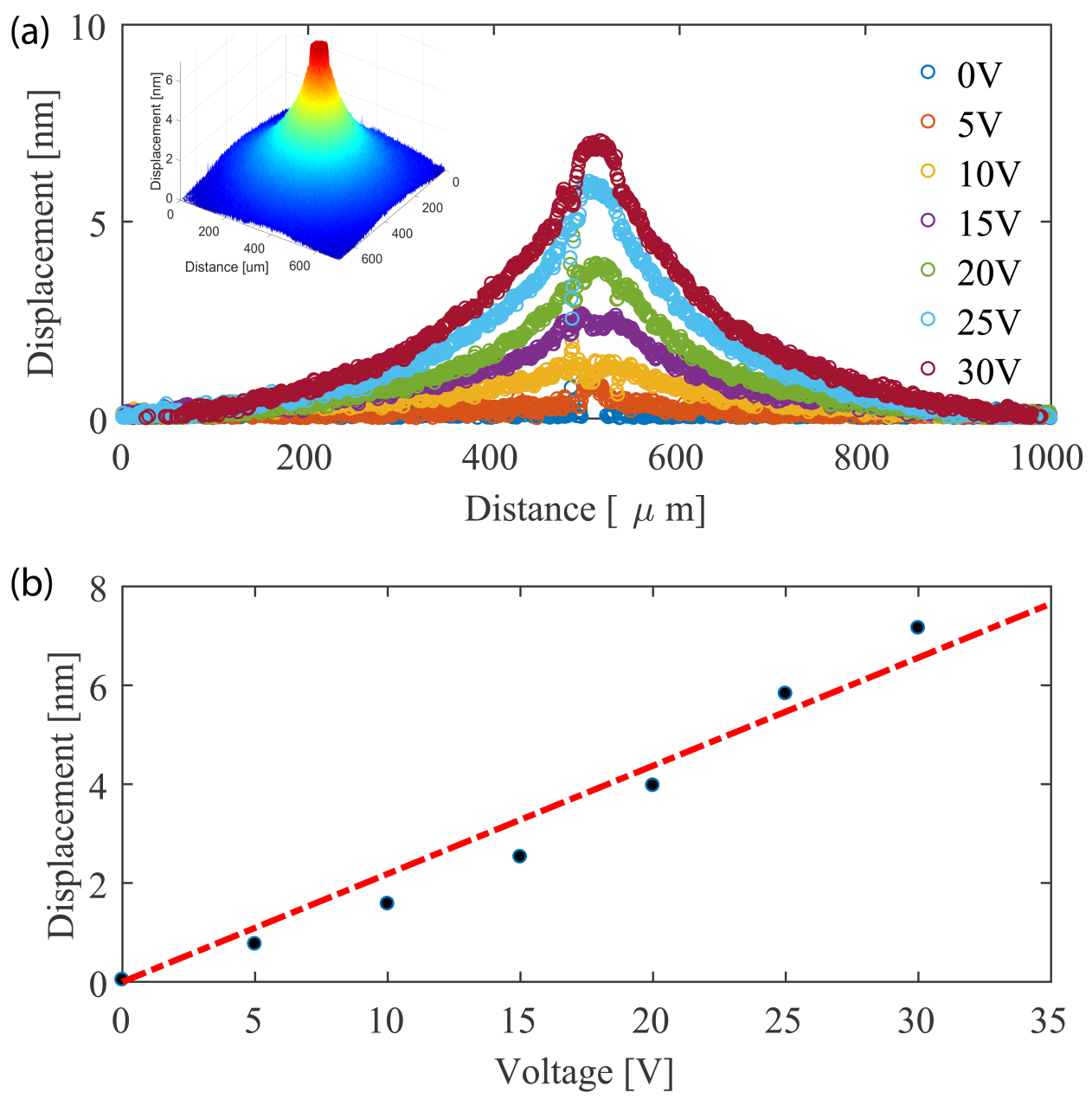

Figure 3.4: (a) Line profiles of a single pixel powered at different voltages showing that the displacement increases with increasing voltage on the pixel as well as the area around it. The inset shows the three dimensional surface profile of a pixel at $30 \mathrm{~V}$ (b) Voltage dependent displacement and a linear fit to the data.

Outside the active region there is no pixel or mediation layer, and therefore the surface displacement could not be varied and controlled. The four corners of the profiles were outside the optical field of WLI and could not be measured. Note that the imprint of the wires is visible in all of the profiles. The wire imprint is not a real surface displacement but a measurement artifact for two reasons: first, the imprint was observed even in the case where all pixels were powered at $0 \mathrm{~V}$ (not shown). Second, the isolation layer was sufficiently isolating the wires from the mediation layer, and the wires were not electrically connected to the mediation layer; only the pixels were powered. The wire imprint was measured as a result of a combination 

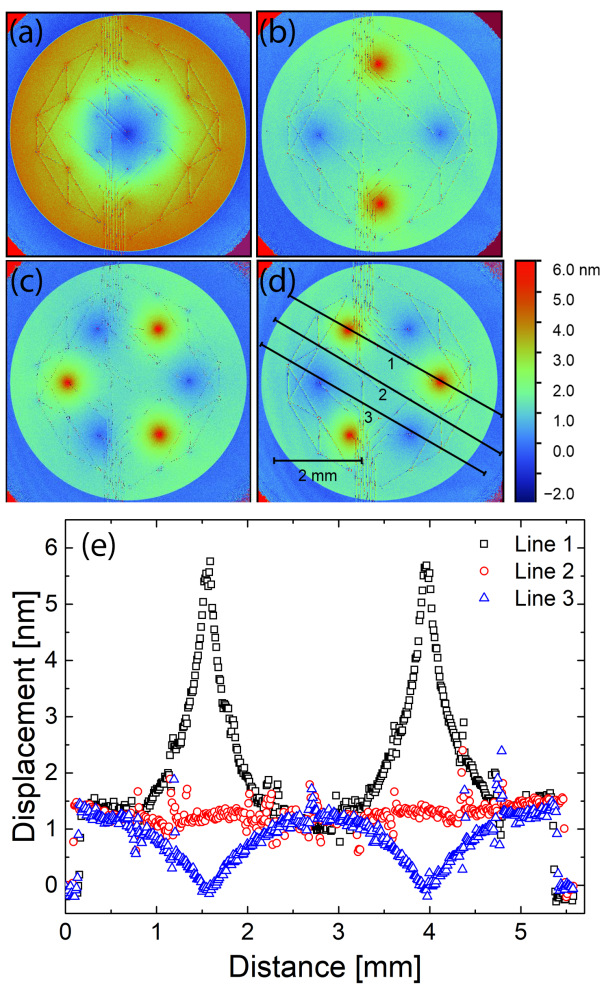

Figure 3.5: (a-d) Generation of surface profiles performed by varying the applied voltages on the pixels: Four surface profiles demonstrating the capability of steering surface profiles. The color scale represents displacement values from $-2 \mathrm{~nm}$ to $6 \mathrm{~nm}$. (e) Three line profiles for the lines shown in (d).

of non-uniform thickness caused by the wire thickness, and an unavoidable sample drift between the main and the baseline measurements. Also note that the noise in Fig. 3.4(a) was due to the same measurement artifact.

In Fig. 3.5(a) the voltages were increased radially from $0 \mathrm{~V}$ to $21 \mathrm{~V}$ with intermediate values of $10 \mathrm{~V}$ and $17 \mathrm{~V}$. As can be seen the result is a radially changing displacement. Another pattern is shown in Fig. 3.5(b). In this surface profile, all pixels except four were powered at $10 \mathrm{~V}$ : the two pixels that are still in blue were at $0 \mathrm{~V}$, and the two red pixels were at $25 \mathrm{~V}$. Fig. 3.5(c) and Fig. 3.5(d) were made opposite to each other. In these two profiles, three pixels were powered at $25 \mathrm{~V}$, three pixels were at $0 \mathrm{~V}$, and the rest of the pixels were powered at $10 \mathrm{~V}$.

Further details of the surface profile can be seen from the three line scans shown in Fig. 3.5(e) that were made from the surface profile in Fig. 3.5(d). Line 1 passes through the two pixels that were powered at $25 \mathrm{~V}$. These two pixels have a maximum displacement of nearly $6 \mathrm{~nm}$. In between these two pixels, the displacement reaches 
a lower value of nearly $1.3 \mathrm{~nm}$. This amount is slightly lower than the expected displacement for $10 \mathrm{~V}(1.7-1.9 \mathrm{~nm})$ just because there is another pixel at $0 \mathrm{~V}$ in its vicinity. The second line profile passes through the middle of the sample where the effective applied voltage is nearly the same across the line. Thus, the displacement in the second line profile is nearly constant along the entire diameter. Finally, the third line profile passes through two pixels that are at $0 \mathrm{~V}$, resulting in no displacement on the two pixels. Again, between these two pixels, the displacement nearly reaches the value for $10 \mathrm{~V}$.

\subsection{Summary}

In summary, we have fabricated for the first time a piezoelectric based functional actuator with gradual surface modulation intended for wavefront correction at XUV and SXR wavelengths. The surface manipulation was performed by independently controlling the voltage of a pixels array. We demonstrated the generation of gradually varying surface profiles by including a resistive mediation layer on the piezoelectric film and in-between the pixels. The mediation layer was capable of producing a continuously varying voltage profile between the pixels. We demonstrated nm-scale displacement suitable for nm-scale corrections of optics for XUV and SXR wavelengths. Nevertheless, the method can potentially provide a high level of actuation up to a few tens of nanometer suitable for the vacuum ultraviolet.

\section{Acknowledgments}

This work is part of the research programme "Smart Multilayer Interactive Optics for Lithography at Extreme UV wavelengths (SMILE)", with contract number 10448 and financial support by the Dutch Research Council (NWO) and Carl Zeiss SMT. The authors acknowledge the support of the Industrial Focus Group XUV Optics, carried out at the MESA + Institute for Nanotechnology at the University of Twente and further support by the Province of Overijssel, ASML, and Malvern Panalytical. We would like to thank Dr. Minh D. Nguyen for the technical support in developing the process flow, and Dr.ir. B. Schurink for PECVD of $\mathrm{SiO}_{2}$. 


\section{References}

[1] W. Chao, B. D. Harteneck, J. A. Liddle, E. H. Anderson, and D. T. Attwood, Soft X-ray microscopy at a spatial resolution better than $15 \mathrm{~nm}$, Nature 435, 1210 (2005).

[2] J. Nordgren and J. Guo, Instrumentation for soft X-ray emission spectroscopy, Journal of Electron Spectroscopy and Related Phenomena 110-111, 1 (2000).

[3] F. G. Eparvier, P. C. Chamberlin, T. N. Woods, and E. M. B. Thiemann, The Solar Extreme Ultraviolet Monitor for MAVEN, Space Science Reviews 195, 293 (2015).

[4] V. Bakshi, EUV lithography, second edition ed. (SPIE Press, Bellingham, Washington USA, 2018).

[5] D. Attwood, Soft X-rays and Extreme Ultraviolet Radiation (Cambridge University Press, Cambridge, 1999).

[6] E. Louis, A. E. Yakshin, T. Tsarfati, and F. Bijkerk, Nanometer interface and materials control for multilayer EUV-optical applications, Progress in Surface Science 86, 255 (2011).

[7] Q. Huang, V. Medvedev, R. van de Kruijs, A. Yakshin, E. Louis, and F. Bijkerk, Spectral tailoring of nanoscale EUV and soft x-ray multilayer optics, Applied Physics Reviews 4, 011104 (2017).

[8] S. K. Ravensbergen, P. Rosielle, and M. Steinbuch, Deformable mirrors with thermo-mechanical actuators for extreme ultraviolet lithography: Design, realization and validation, Precision Engineering 37, 353 (2013).

[9] J. Walker, T. Liu, M. Tendulkar, D. N. Burrows, C. T. DeRoo, R. Allured, E. N. Hertz, V. Cotroneo, P. B. Reid, E. D. Schwartz, T. N. Jackson, and S. TrolierMcKinstry, Design and fabrication of prototype piezoelectric adjustable X-ray mirrors, Optics express 26, 27757 (2018).

[10] R. Saathof, G. J. M. Schutten, J. W. Spronck, and R. H. Munnig Schmidt, Actuation profiles to form Zernike shapes with a thermal active mirror, Optics Letters 40, 205 (2015).

[11] F. Yang, M. Li, L. Gao, W. Sheng, P. Liu, and X. Zhang, Laser-heating-based active optics for synchrotron radiation applications, Optics Letters 41, 2815 (2016).

[12] M. Bayraktar, A. Chopra, G. Rijnders, K. Boller, and F. Bijkerk, Wavefront correction in the extreme ultraviolet wavelength range using piezoelectric thin films, Optics express 22, 30623 (2014).

[13] J. Jiang, S.-G. Hur, and S.-G. Yoon, Epitaxial PMN-PT Thin Films Grown on $\mathrm{LaNiO}_{3} / \mathrm{CeO}_{2} / \mathrm{YSZ}$ Buffered Si(001) Substrates by Pulsed Laser Deposition, Journal of The Electrochemical Society 158, G83 (2011). 
[14] N. Bassiri-Gharb, S. Trolier-McKinstry, and D. Damjanovic, Strain-modulated piezoelectric and electrostrictive nonlinearity in ferroelectric thin films without active ferroelastic domain walls, Journal of Applied Physics 110, 124104 (2011).

[15] A. Chopra, M. Bayraktar, F. Bijkerk, and G. Rijnders, Controlled growth of $\mathrm{PbZr}_{0.52} \mathrm{Ti}_{0.48} \mathrm{O}_{3}$ using nanosheet coated Si (001), Thin Solid Films 589, 13 (2015).

[16] M. D. Nguyen, E. P. Houwman, M. Dekkers, and G. Rijnders, Strongly Enhanced Piezoelectric Response in Lead Zirconate Titanate Films with Vertically Aligned Columnar Grains, ACS applied materials \& interfaces 9, 9849 (2017).

[17] A. Chopra, M. Bayraktar, M. Nijland, J. E. ten Elshof, F. Bijkerk, and G. Rijnders, Tuning of large piezoelectric response in nanosheet-buffered lead zirconate titanate films on glass substrates, Scientific reports 7, 251 (2017). 



\section{4}

\section{Influence of template layer on structure and ferroelectric properties of $\mathrm{PbZr}_{0.52} \mathrm{Ti}_{0.48} \mathrm{O}_{3}$ films}

The effect of the deposition conditions of the template $\mathrm{LaNiO}_{3}$ layer on the microstructure and ferroelectric properties of the subsequently deposited thin film of $\mathrm{PbZr}_{0.52} \mathrm{Ti}_{0.48} \mathrm{O}_{3}(\mathrm{PZT})$ is investigated. The explored deposition conditions were the deposition pressure of $\mathrm{O}_{2}(0.1$ to $0.8 \mathrm{mbar})$ and thickness (100 and $200 \mathrm{~nm}$ ) of the template layer that is deposited on $\mathrm{Ca}_{2} \mathrm{Ni}_{3} \mathrm{O}_{10}$ nanosheet-covered Si substrates. Both increasing the deposition pressure and thickness of the template layer changes the growth of PZT from a smooth dense film into a rough columnar film. We explain the change in PZT microstructure by an increase in the roughness of the template layer to accommodate strain relaxation. The ferroelectric properties of the columnar films are changed compared to the smooth dense films. We conclude that this results from the growth of PZT in more evenly distributed and smaller lateral grains in the columnar case as compared to the dense, smooth film case. The ability to tune the ferroelectrics properties with the microstructure is primarily relevant for ferroelectric applications such as actuators and systems for energy harvesting and storage. 


\subsection{Introduction}

Ferroelectric thin films are of high interest for applications such as in ferroelectric memories, or in microelectromechanical systems as sensors and actuators. [1-8] Especially $\mathrm{PbTi}_{1-x} \mathrm{Zr}_{x} \mathrm{O}_{3}$ (PZT) is favored due to its high ferroelectric and piezoelectric properties. These functional properties of PZT thin films are highly dependent on several material properties, such as stoichiometry, crystal orientation and microstructure. The most favorable properties of PZT are found in the $x=0.48$ morphotropic phase boundary (MPB) composition [9-12]. It has been shown theoretically [13, 14] and experimentally [15-17] that the PZT grown in (001)-orientation, the out-of-plane direction, exhibits higher functional response compared to other orientations, hence the PZT films have been initially grown on lattice-matched $\mathrm{SrTiO}_{3}$ substrates to promote such oriented growth [18]. To grow (001)-oriented PZT on affordable substrates such as Si, glass or metals, several buffer layers have been explored [19-23]. In particular, it has been shown that chemically-synthesized unit-cell-thick oxide nanosheets can be used for achieving the desired out-of-plane crystal orientation on silicon and glass substrates [24-29]. Given the right stoichiometry and orientation, ferroelectric response is further affected by the microstructure of the film, that is affected by the grain size and domain structure [3, 30, 31]. The film microstructure also influences the application relevant piezoelectric properties by influencing the elastic parameters of the film [32-38]. Therefore, it is important to explore the methods that allow controlling film microstructure and consequent influences on the film's functional properties.

The influence of the microstructure on the functional properties of the deposited films has been studied using various deposition techniques. Investigations in chemical solution deposited PZT films show that dense films can be produced by process optimization [39] and multiple step deposition [40]. In magnetron sputtered PZT films, dense films have been reported on matching template layers [41-44] and a columnar microstructure has been reported for fast-cooled low temperature depositions [45]. Pulsed laser deposition (PLD) is a proven technique for high-crystalquality growth with an important additional parameter, i.e. the laser repetition frequency. It has been shown that low laser repetition frequency helps in producing dense films and increasing the frequency can convert the film microstructure from dense to columnar $[36,38]$. To the best of our knowledge, the change of microstructure and ferroelectric properties was so far only explored with regards to the deposition conditions of the PZT, even though it is well-known that the template layer has an influence on the microstructure, and thus also on the ferroelectric properties of the subsequent layers.

From the application point of view, it is known that the film microstructure influences the ferroelectric and piezoelectric properties. In energy storage devices for example, a low remnant and high maximum polarization in combination with minimal hysteresis is desired to increase the amount of recoverable energy [46]. As these properties are among other things dependent on the film microstructure, investigating the influence of the template layer on the ferroelectric film in terms 
of microstructure and ferroelectric properties is of interest. The influence of the template layer is also of interest for energy harvester devices, as the piezoelectric properties of the film are influenced by the microstructure. Any improvement of the piezoelectric properties can directly lead to an improvement of the figures of merit of the energy harvesting device [47, 48]. Furthermore, the ability to control the mode of operation between bending type ( $d_{31}$ mode) and piston type ( $d_{33}$ mode) by tuning the microstructure, is attractive for modern microelectromechanical actuators $[4,17,49]$.

Here, we investigate the effect of the deposition conditions of the template layer, $\mathrm{LaNiO}_{3}$ (LNO), on the microstructure and ferroelectric properties of the subsequently deposited PZT layer. The explored deposition condition were the gas pressure and the thickness of the template layer while keeping the subsequent PZT deposition conditions, including the laser repetition frequency, constant for all samples.

\subsection{Experimental details}

\subsubsection{Film deposition and fabrication}

All samples were deposited by PLD with a KrF excimer Laser (Lambda Physik, wavelength $248 \mathrm{~nm}$, pulse duration $20 \mathrm{~ns}$ ) on Si substrates that are pre-coated with $\mathrm{Ca}_{2} \mathrm{Nb}_{3} \mathrm{O}_{10}(\mathrm{CNO})$ nanosheets using Langmuir-Blodget deposition, to achieve (001)orientation of the samples [24, 28, 50]. All depositions were performed in a chamber with a base pressure below $2.5 \times 10^{-5}$ mbar. During the deposition, the substrate temperature was kept constant at $600^{\circ} \mathrm{C}$. For all samples the LNO was ablated from a stoichiometric target (Kurt J. Lesker) with a laser spot size of $3 \mathrm{~mm}^{2}$, a fluence of $2.25 \mathrm{~J} \mathrm{~cm}^{-2}$, a repetition frequency of $4 \mathrm{~Hz}$, and a substrate to target distance of $60 \mathrm{~mm}$. Four sets of samples were fabricated by varying the $\mathrm{O}_{2}$ pressure (set-A), introducing a buffer gas (set-B), increasing the thickness of the LNO in the LNO/PZT heterostructure (set-C), and for studying the LNO template layer in detail sole LNO layers (set-D), as summarized in Tab. 4.1. The starting $\mathrm{O}_{2}$ pressure during the LNO deposition was taken from literature [38] and the maximum pressure of 0.8 mbar was the limit of the experimental setup. The starting thickness of LNO layers was selected around $100 \mathrm{~nm}$, a common thickness for oxide electrodes for thick PZT films [31]. The PZT was deposited subsequent to LNO deposition without breaking the vacuum. The PZT layer was deposited from a MPB $\mathrm{PbZr}_{0.52} \mathrm{Ti}_{0.48} \mathrm{O}_{3}$ ceramic target (self-made) at a pressure of $0.1 \mathrm{mbar}$, laser spot size of $3 \mathrm{~mm}^{2}$, fluence of $2.5 \mathrm{~J} \mathrm{~cm}^{-2}$, repetition frequency of $50 \mathrm{~Hz}$, and a substrate to target distance of $55 \mathrm{~mm}$ as in literature. [38] After the deposition the samples were cooled down to room temperature at a cooling rate of $8^{\circ} \mathrm{C} \mathrm{min}^{-1}$ in $1 \mathrm{mbar}_{2}$ pressure. In the set-D samples, only the lowest and highest range of the parameters were investigated. For ferroelectric measurements of the LNO/PZT heterostructures (sets-A, B and C) a $100 \mathrm{~nm}$ thick platinum (Pt) layer was deposited on top of the PZT layer by sputtering. The deposited Pt layer was patterned and structured into $200 \times 200 \mathrm{\mu m}^{2}$ top electrodes using a standard photolithography lift-off process. 
The bottom electrode was contacted by use of silver glue at the side of the sample.

\subsubsection{Analysis and characterization}

Crystallographic properties of sets-A, B and C were analyzed using $\theta-2 \theta$ scans with an X-ray diffractometer (XRD) system (PANalytical X'Pert). The film surface was investigated by means of atomic force microscopy (AFM, Bruker Dimension ICON) and the root-mean-squared (RMS) surface roughness was calculated. The RMS roughness values for sets- $\mathrm{A}, \mathrm{B}$ and $\mathrm{C}$ correspond to the average value taken from five $1 \times 1 \mathrm{\mu m}^{2}$ boxes on the smooth nanosheet-coated areas. The AFM images of set-D were covering only nanosheet-coated $1.5 \times 1.5 \mathrm{\mu m}^{2}$ areas and the RMS values were calculated over the complete area. The cross-sectional microstructure and film thicknesses were investigated by high-resolution scanning electron microscopy (HRSEM, Zeiss Merlin 1550). The polarization hysteresis measurements were performed with a ferroelectric analyzer (AixACCt TF-3000) using a triangular AC-electric field of $\pm 200 \mathrm{kV} \mathrm{cm}^{-1}$ amplitude with a frequency of $1 \mathrm{kHz}$. The wake-up behavior, which will be explained in the ferroelectric properties section, was measured using a rectangular AC-field of $\pm 200 \mathrm{kV} \mathrm{cm}^{-1}$ amplitude with a frequency of $10 \mathrm{~Hz}$ for cycling the samples $10^{4}$ times.

\subsection{Results and discussion}

\subsubsection{Microstructure of PZT and LNO}

The first parameter that was investigated with AFM and SEM analysis is the oxygen pressure (set-A) during the LNO deposition. Fig. $4.1 \mathrm{a}-\mathrm{d}$ ) show the surface topography (top row AFM) and microstructure change in the cross-section of the PZT (bottom row SEM) while increasing the $\mathrm{O}_{2}$ pressure from 0.1 mbar to 0.8 mbar. The AFM images show that relatively smooth, wide areas are visible at low $\mathrm{O}_{2}$ pressures and the roughness in these wide areas increase with increasing $\mathrm{O}_{2}$ pressure. Boundaries of more than $200 \mathrm{~nm}$ height in between the wide areas correspond to the gaps in between the individual nanosheet flakes where the PZT exhibits a (110)-oriented growth with higher growth rate than (001)-orientation [28]. In the following, we only compare the changes that occur on top of the CNO nanosheetcoated areas covering over $95 \%$ of the sample, neglecting the gap areas. The RMS roughness in the CNO nanosheet-coated areas increases 3 times $(7.2 \mathrm{~nm}$ to $23.4 \mathrm{~nm})$ with increasing $\mathrm{O}_{2}$ pressure, see Tab. 4.1. The cross-sectional SEMs show that with increasing oxygen pressure, the PZT starts to grow more columnar and the columnar microstructure becomes visible earlier in the growth direction of the PZT film. For low $\mathrm{O}_{2}$ pressure, the columns are only present at the top $50 \mathrm{~nm}$ to $100 \mathrm{~nm}$ of the PZT film whereas for the highest $\mathrm{O}_{2}$ pressure the PZT film grows in columns after half of the thickness and the top $500 \mathrm{~nm}$ is columnar. Overall, the trend towards columnar microstructure with increasing $\mathrm{O}_{2}$ pressures is evident. On the other hand, this parameter scan does not give any idea if the total pressure, or the $\mathrm{O}_{2}$ partial pressure (p.p.) is playing the dominant role in the microstructure change. For clarification, in set-B the total pressure is kept at 0.8 mbar, same as the highest oxygen pressure in set-A, while the oxygen and argon partial pressures are 


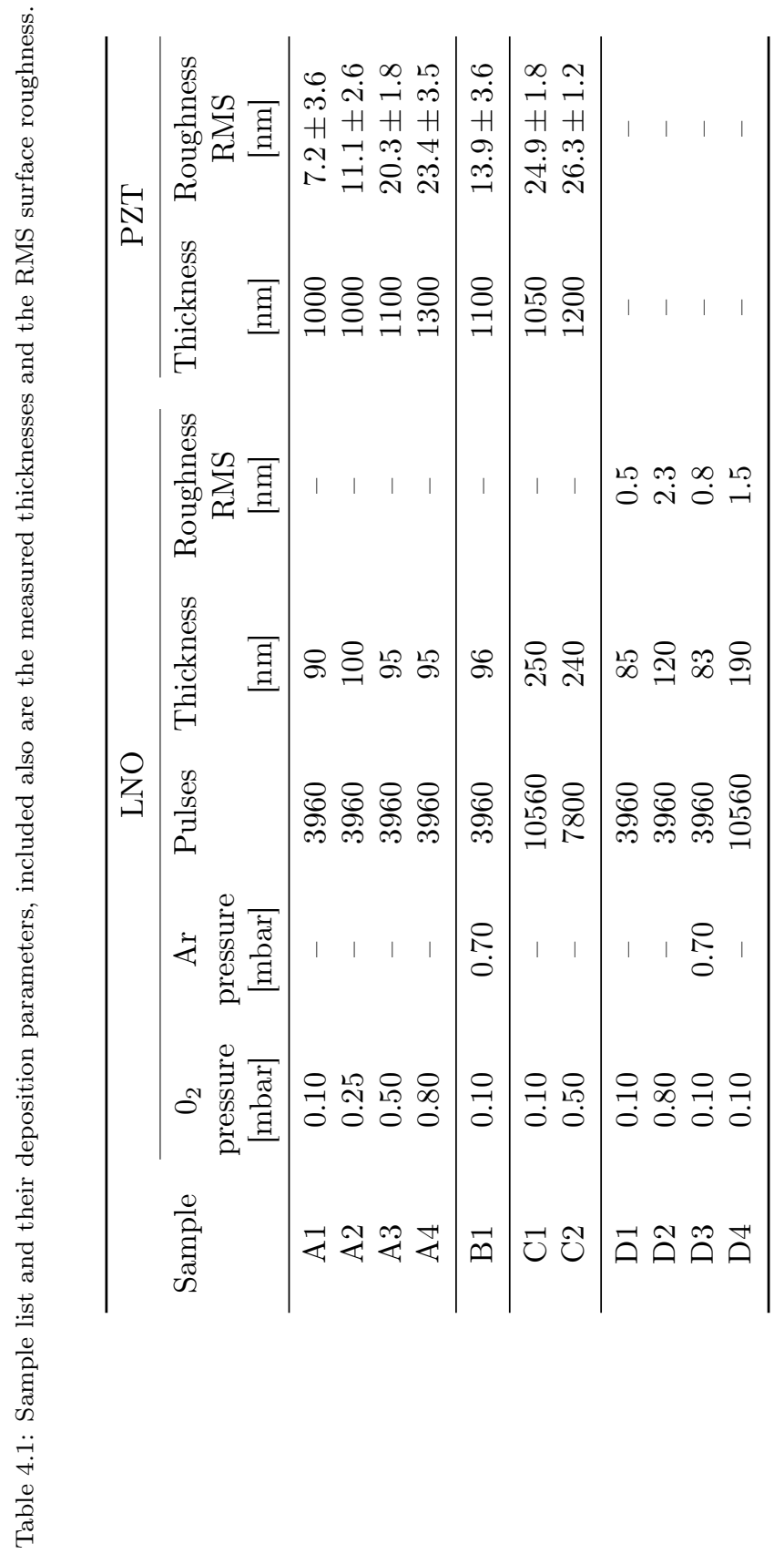


set to 0.1 and 0.7 mbar, respectively. This set allows to distinguish the influences of the pressure of the $\mathrm{O}_{2}$, which can chemically interact with the deposited film, and the inert Ar. The results in Fig. 4.1 e) show that the sample surface is by a factor of 2 less rough than for sample A4 deposited at 0.8 mbar $\mathrm{O}_{2}$ pressure, see Tab. 4.1. The cross-sectional SEM also shows that the bulk of the PZT film is dense, only the top part shows a few columns similar to the low $\mathrm{O}_{2}$ pressure depositions.

The next parameter that was investigated is the thickness of the LNO layer. The LNO layer thickness was increased by 2.5 times in set-C for 0.1 and 0.5 mbar $\mathrm{O}_{2}$ pressures. To be compared are the samples A1 to C1 (Fig. 4.1 a) to Fig. $4.1 \mathrm{f}$ )), and $\mathrm{A} 3$ to $\mathrm{C} 2$ (Fig. $4.1 \mathrm{c}$ ) to Fig. $4.1 \mathrm{~g}$ )). At low 0.1 mbar $\mathrm{O}_{2}$ pressure, increasing the thickness results in 3 times rougher PZT film surface: the RMS roughness increases from $7.2 \mathrm{~nm}$ to $24.9 \mathrm{~nm}$. This increase of roughness is due to the change of PZT microstructure from a completely dense, smooth film to a film that grows in separated columns at the top third part of its total thickness. The comparison of the effect of thickness at an $\mathrm{O}_{2}$ pressure of 0.5 mbar, shows the same trend but with less drastic change. The RMS roughness increases from $20.3 \mathrm{~nm}$ to $26.3 \mathrm{~nm}$ only. This can be expected if one considers that the PZT surface roughness should reach a saturation when the top of the film grows in fully separated columns, and all the columns have the same diameter and tip shape. The last observation from Fig. 4.1 and Tab. 4.1 is the thickness difference between the deposited PZT films, even though their deposition conditions are the same. The more columnar the film, the higher is the thickness. This can be explained considering the stacking of material in the PZT films. The amount of deposited material is the same but due to the voids between the columns, more material is preferentially stacked in the growth direction on top of the columns.

Up to here we have analyzed the PZT layer to identify the effect of deposition conditions of the template layer. To better investigate the template layer itself, we deposited and analyzed the template layer in set-D without adding the PZT layer. To minimize the number of needed samples, only the two extremes of the $\mathrm{O}_{2}$ pressure (0.1 and 0.8 mbar) (D1-D2), the deposition at 0.7 mbar p.p. Ar plus 0.1 mbar p.p. $\mathrm{O}_{2}$ pressure (D3), and the deposition at 0.1 mbar $\mathrm{O}_{2}$ pressure with doubled LNO thickness (D4) were investigated. Low $\mathrm{O}_{2}$ pressure deposition, sample D1, shown in Fig. $4.2 \mathrm{a}$ ) has a very smooth LNO surface with the lowest RMS roughness, $0.5 \mathrm{~nm}$. The area scanned with AFM in this set is much smaller than the set-A-B-C samples, only spanning a single nanosheet flake, hence gaps regions are not visible. The SEM cross-section shows that the LNO grows mostly dense with round grains visible only at the surface. The sample grown at $0.8 \mathrm{mbar} \mathrm{O}_{2}$ pressure (D2) has an increased surface RMS roughness of $2.3 \mathrm{~nm}$. The SEM cross-section, Fig. $4.2 \mathrm{~b}$ ), shows that the LNO grows less dense and constituted by columnar LNO grains. The sample grown with 0.1 mbar $\mathrm{O}_{2}$ p.p. and 0.8 mbar total pressure (D3, shown in Fig. $4.2 \mathrm{~d}$ ) has a surface roughness of $0.8 \mathrm{~nm}$, slightly increased in comparison to the surface roughness of 0.1 mbar sample, D1 in Fig. $4.2 \mathrm{a}$ ). These two samples show no clear difference in microstructure as seen in the SEM pictures. Increasing the LNO thickness to $190 \mathrm{~nm}$ at $0.1 \mathrm{mbar}_{2} \mathrm{O}_{2}$ pressure (D4), increases the surface roughness 

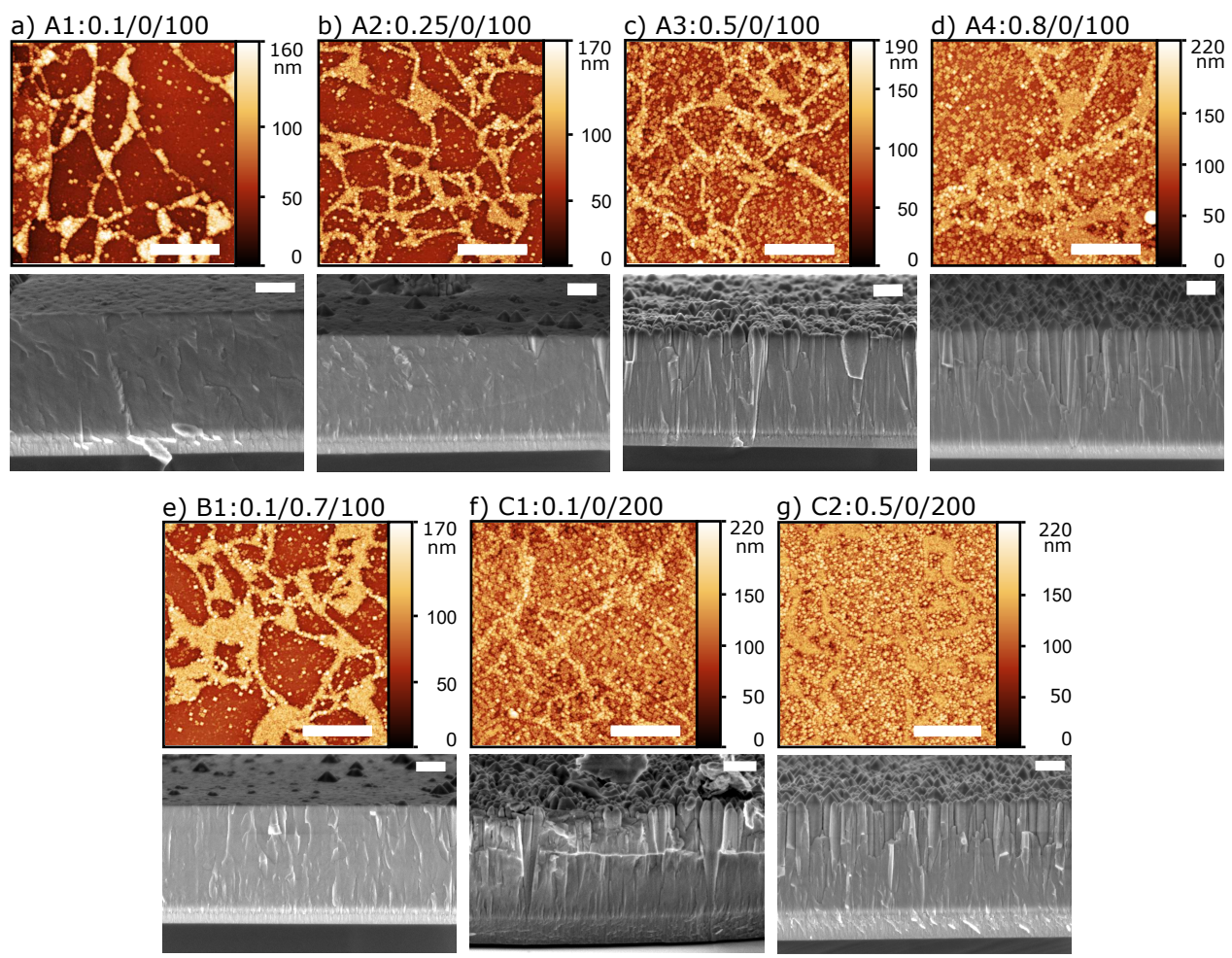

Figure 4.1: AFM surface scans and SEM cross-sections for LNO/PZT samples. The deposition conditions are given at the top of each subfigure with the following coding. Sample ID: $\mathrm{O}_{2}$ p.p./Ar p.p./LNO thickness [nm]. The scale bar in the AFM represents $3 \mu \mathrm{m}\left(10 \times 10 \mathrm{~mm}^{2}\right.$ area $)$ and in the SEM corresponds to $300 \mathrm{~nm}$.

by a factor 3, compare Fig. 4.2 a) and Fig. 4.2 d). The cross-sectional SEMs show that the grain size of the LNO is increased for the thicker LNO.

We explain the increasing trend in the columnar PZT microstructure in Fig. 4.1 by the increase in roughness of the template LNO layer due to either an increase in the deposition pressure or in the thickness of the LNO layer. This roughness increase leads to a reduced diffusion distance of the deposited PZT and results into a columnar growth. According to the literature, the diffusion time for the PZT is low at high deposition frequency of $50 \mathrm{~Hz}$ and hence columnar growth is promoted [28]. However, in contrast to literature [28], we obtained non-columnar, dense and smooth films as well at literature's deposition conditions including the $50 \mathrm{~Hz}$ deposition frequency of PZT. Our results show that in addition to the high laser repetition frequency, the roughness of the template also plays a crucial role. In the literature, there is also disagreement between experimental findings [28] and theoretical predictions [51] regarding the influence of the deposition frequency in PLD. Experiments show very clearly that the PZT grows more columnar at higher repetition frequencies [28]. The theoretical study, which assumes independence of the growth mode from the deposited material, predicts that the material should 


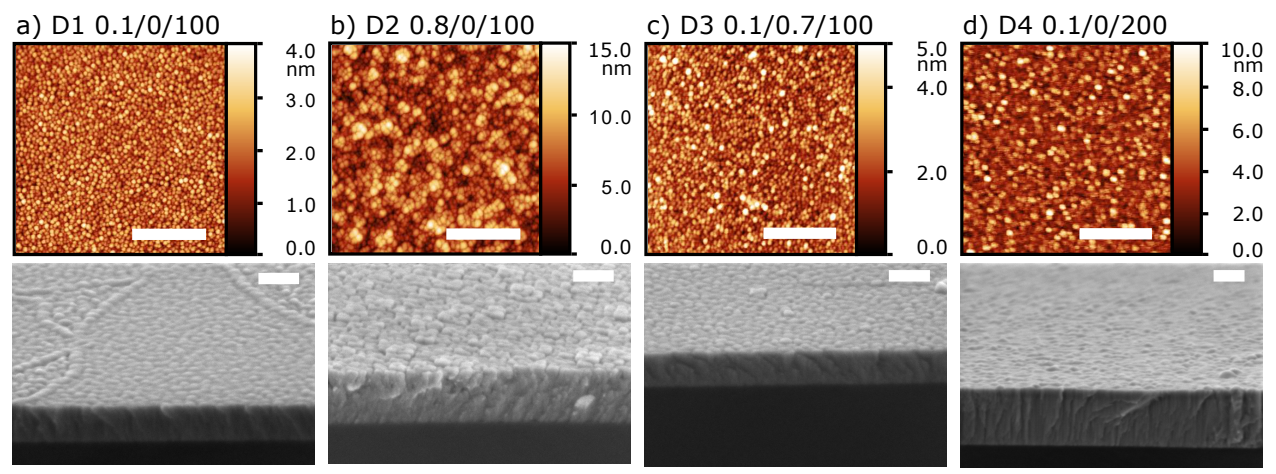

Figure 4.2: AFM surface scans and SEM cross-sections for LNO samples. The deposition conditions are given at the top of each subfigure with the coding Sample ID: $\mathrm{O}_{2}$ p.p./Ar p.p./LNO thickness [nm]. The scale bar in the SEM corresponds to $100 \mathrm{~nm}$ and in the AFM to $500 \mathrm{~nm}$ $\left(1.5 \times 1.5 \mathrm{\mu m}^{2}\right.$ area $)$.

grow in a smooth film at high deposition frequencies due to reduced size of the nucleating islands and enhanced diffusion of adatoms from the top of the growing islands to the substrate [51]. Our observation of a dense film at the high deposition frequency can help to solve the disagreement of these findings. If the growth template is smooth, the grown film is smooth as theoretically predicted [51], whereas in the case of a rough template, the diffusion of adatoms does not take place and the material starts to grow in separated columns, especially at higher frequencies.

Another finding based on set-B was that not the total pressure, but the oxygen partial pressure is the deciding factor in determining the roughness and microstructure of the LNO layer. The microstructure of $\mathrm{B} 1$, with the $0.1 \mathrm{mbar}_{2} \mathrm{O}_{2}$ p.p. and 0.8 mbar total pressure, is more comparable to sample grown at 0.25 mbar $\mathrm{O}_{2}$ pressure, but not to the sample grown at $0.8 \mathrm{mbar} \mathrm{O}_{2}$ pressure. This observation points out that the dominant parameter is not the kinetic energy of the ablated material but the interaction of the ablated species with the reactive $\mathrm{O}_{2}$ gas. For higher oxygen pressure, the ablated material seems to interact more with the oxygen and form clusters of multiple unit-cells in the plasma plume [52]. If these clusters are deposited on the substrate, we do not have the unit-cell by unit-cell build up known from epitaxial films, but a cluster-by-cluster growth. These clusters have a lower mobility and form rough islands as seeds for further columnar growth.

\subsubsection{Crystal structure}

The out-of-plane crystal orientation of the PZT samples, sets-A-B-C, were characterized by XRD $\theta-2 \theta$ scans as shown in Fig. 4.3 a). Both PZT and LNO grows mainly (001)-oriented with small (110) and (111) peaks. According to literature, the (110) and (111) peaks can be attributed to the growth in the gap regions between the nanosheets, directly on the Si substrate [37, 38, 53]. The Pt (111) peak in sample $\mathrm{C} 1$ is due to measurement of the sample only after the top Pt electrode structuring. The changes in the deposition conditions of the LNO template do not 
seem to visibly influence the crystallinity of the atop grown PZT. The LNO (002) peaks show some changes with the deposition conditions, more clearly visible in Fig. $4.3 \mathrm{~b}$ ). One observes that the LNO (002) peak is showing a broad, double peak structure and is shifted to higher angles for increasing oxygen pressure and higher LNO film thickness. The $2 \theta$ angles of the individual peaks in this double peak structure are around the in-plane lattice parameters of CNO $\left(47.088^{\circ}\right)[24,25]$ and the bulk pseudo-cubic lattice parameter of LNO $\left(47.348^{\circ}\right)[35,54]$. Describing our LNO film with a pseudo-cubic lattice of equal in-plane and out-of-plane lattice parameters, these double peaks point to two crystal structures with two different inand out-of-plane lattice parameters. It is conceivable that the bottom part of the LNO grows epitaxially strained to the CNO nanosheet, resulting in the first XRD peak matching the CNO lattice parameter at lower $2 \theta$ angle. The top part of the LNO however grows relaxed to the bulk lattice parameter of LNO, resulting in the second observed peak at higher $2 \theta$ angle. Therefore, with increasing $\mathrm{O}_{2}$ pressure and thickness the LNO grows less strained to the CNO nanosheet and relaxes to the bulk LNO lattice parameters.

The observed relaxation with increasing film thickness was reported for an LNO layer that was sputtered directly on Si substrate and attributed to the relaxation of the thermal strain [54]. In another report about the LNO films deposited on $\mathrm{LaAlO}_{3}$ substrates, decreasing $\mathrm{O}_{2}$ pressure produces less strained films due to incorporation of the oxygen vacancies [55]. However, both of these reports do not present a double peak structure for the LNO, but only a shift of the LNO peak. To explain the observed double peak structures, the relaxation of thermal strain is not sufficient, as the thermal strain would be same for the whole LNO film and would not lead to a splitting of the peak. We attribute our observations rather to epitaxial strain relaxation. For low $\mathrm{O}_{2}$ pressure and a thickness of about $100 \mathrm{~nm}$ the LNO grows epitaxially strained to the $\mathrm{CNO}$ nanosheet, and further increase in $\mathrm{O}_{2}$ pressure or thickness leads to roughening of the film as observed in sample set D to release the strain.

To quantify the strained and relaxed fractions of the LNO films, we fitted the (002) peaks of all samples in the $46^{\circ}$ to $48.5^{\circ}$ range with two Voigt functions after subtracting a linear background. The fitting for sample A4 is shown in Fig. 4.3 c) as an example. Tab. 4.2 gives the central positions, widths (FWHM), areas and area ratios of the fitted peaks. For all the samples, the positions of the first and second peaks are matching the expected $47.088^{\circ}$ and $47.348^{\circ}$, respectively, when the $0.15^{\circ}$ systematic error bars are taken into account. The large systematic error-bars in the peak positions are due to difficulty in the alignment. The samples were aligned with respect to the $\mathrm{Si}(004)$ peak. However, the LNO/PZT grows on the nanosheets that are not coupled to the crystal orientation of Si, leading to peak shifts of $\pm 0.15^{\circ}$. In addition to this, the samples with low $\mathrm{O}_{2}$ pressure $(\mathrm{A} 1, \mathrm{~A} 2, \mathrm{~B} 1)$ show larger peak shifts from the bulk values. We attribute these shifts to numerical inaccuracy of the fits due to very small size of the second peaks. 

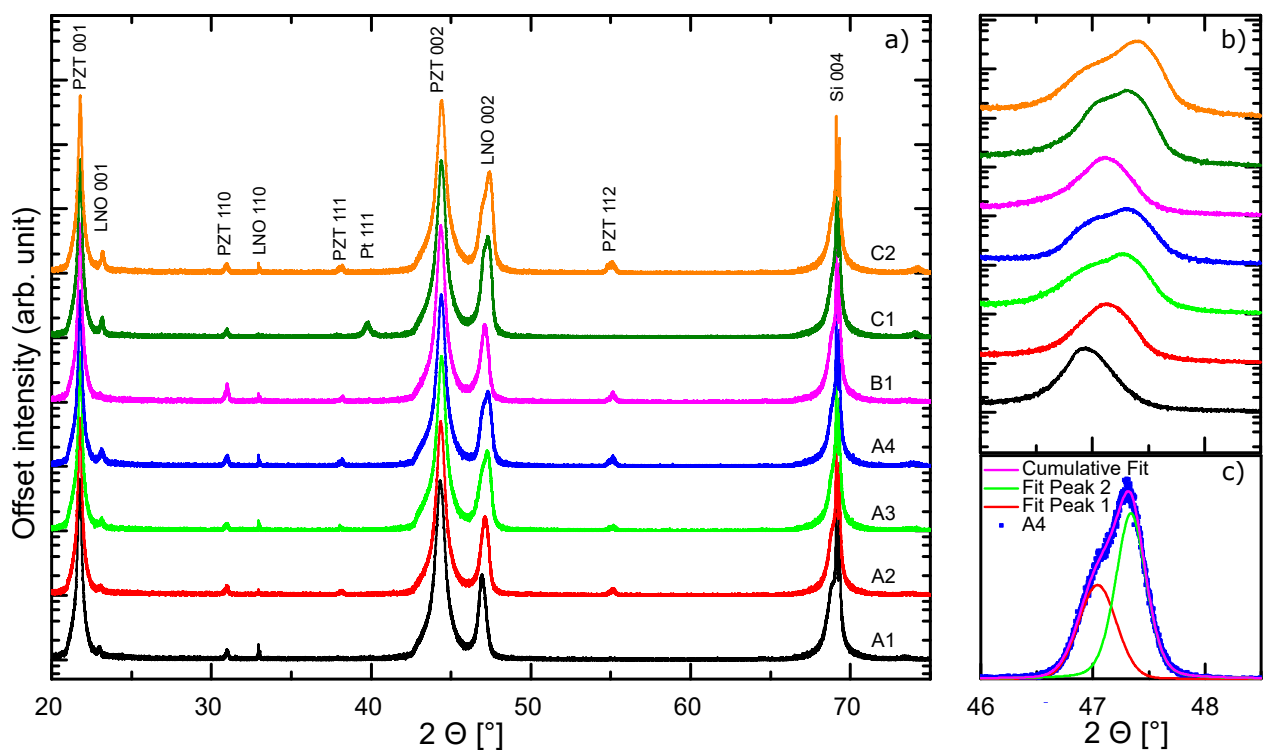

Figure 4.3: a) XRD survey spectra of the LNO/PZT samples. b) Detailed view of the LNO (002) peaks. c) Fitting of LNO (002) peaks of sample A4 with two Voigt functions.

Table 4.2: Peak position, width (FWHM), area and area ratio of the fitted Voigt functions for all $\mathrm{LNO} / \mathrm{PZT}$ samples.

\begin{tabular}{|c|c|c|c|c|c|c|c|}
\hline \multirow[b]{2}{*}{ Sample } & \multicolumn{3}{|c|}{ Peak 1} & \multicolumn{3}{|c|}{ Peak 2} & \multirow[b]{2}{*}{$\frac{\text { Area }(\text { Peak } 2)}{\text { Area }(\text { Peak } 1)}$} \\
\hline & $\begin{array}{c}\text { Position } \\
{\left[{ }^{\circ}\right]} \\
\end{array}$ & $\begin{array}{c}\text { FWHM } \\
{\left[{ }^{\circ}\right]} \\
\end{array}$ & Area & $\begin{array}{c}\text { Position } \\
{\left[^{\circ}\right]} \\
\end{array}$ & $\begin{array}{c}\text { FWHM } \\
{\left[{ }^{\circ}\right]}\end{array}$ & Area & \\
\hline $\mathrm{A} 1$ & 46.95 & 0.31 & 750 & 47.14 & 0 & 0 & $0.0 \pm 0$ \\
\hline $\mathrm{A} 2$ & 47.06 & 0.31 & 468 & 47.22 & 0.25 & 184 & $0.39 \pm 0.03$ \\
\hline A3 & 47.06 & 0.26 & 457 & 47.31 & 0.30 & 371 & $0.81 \pm 0.21$ \\
\hline A4 & 47.04 & 0.39 & 270 & 47.34 & 0.23 & 426 & $1.6 \pm 0.15$ \\
\hline B1 & 47.05 & 0.29 & 469 & 47.20 & 0.23 & 157 & $0.33 \pm 0.02$ \\
\hline $\mathrm{C} 1$ & 47.05 & 0.33 & 699 & 47.32 & 0.30 & 1008 & $1.4 \pm 0.27$ \\
\hline $\mathrm{C} 2$ & 47.00 & 0.36 & 459 & 47.38 & 0.33 & 1238 & $2.7 \pm 0.20$ \\
\hline
\end{tabular}

\subsubsection{Discussion on growth mode}

It is clear from the SEM pictures in Fig. 4.1 and Fig. 4.2, and the extracted roughness values in Tab. 4.1) that roughness of the PZT layer is a good indicator of the level of columnarity of the film. Besides roughness of the PZT, it is instructive to search for quantified indicators in the LNO template layer to better understand and connect the growth mechanism to the template layer. Considering that the epitaxial strain is relaxed by increasing the roughness, we hypothesize that the area ratio of the relaxed to strained part of the LNO layer, Area(Peak2)/Area(Peak1), 
can be such an indicator. It is possible then to check the correlation of the PZT surface roughness and the LNO peak-area ratio as plotted in Fig. 4.4. One observes that there is a high correlation between the peak-area ratio and the PZT surface roughness. For small peak ratios, the RMS roughness is small, showing that the PZT growth is dense. All samples with a peak-area ratio above 0.8 shows high RMS roughness above $20 \mathrm{~nm}$, where the PZT grows columnar verified by the SEM images.

We propose that for the growth of the samples with a peak-area ratio below 0.8, the PZT molecules arriving from the target prefer to assemble epitaxially on the LNO via Frank-van der Merwe growth, a layer-by-layer growth [56]. For this growth mode, the specific surface free energy of the LNO-vacuum interface has to be larger than that of LNO-PZT and PZT-vacuum interfaces. For the samples with a peak-area ratio above 0.8 , the growth of PZT is at the beginning a layer by layer growth. However, at a critical thickness, due to the increased roughness originating from the LNO template, the formation of coherent 3D islands is preferred. The 3D island growth significantly reduces the surface energy due to elastic relaxation of the strain field in islands via Volmer-Weber growth [57]. The combination of starting layer by layer growth with the formation of 3D islands after a certain thickness is referred as Stranski-Krastanov growth model [58]. Further arriving PZT molecules preferentially grow on the $3 \mathrm{D}$ islands due to energy reduction, leading to the well oriented columnar growth at the top of the film that we observe. The resulting roughness is much higher than layer-by-layer growth and also reaches a saturation. The saturation in roughness can be understood considering that the PZT surface roughness will not increase further if the top of the film grows completely columnar, and all the columns have the same diameter and tip shape. The level of saturation occurs above $25 \mathrm{~nm}$ for the investigated PZT films, but it may vary for different materials.

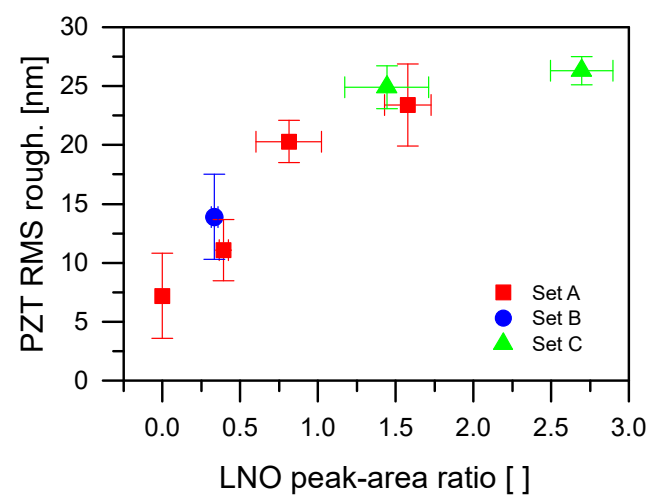

Figure 4.4: RMS roughness of the PZT against the peak-area ratio of the LNO for all LNO/PZT samples. 


\subsubsection{Ferroelectric properties}

In the following the influence of the deposition conditions of the LNO template layer on the ferroelectric properties of the PZT film is investigated. Literature shows that the electrical properties of LNO films in the range of our deposition pressures and thicknesses do not change significantly $[54,55]$. Therefore, the observed changes in the ferroelectric properties can be attributed to either the electrode-PZT interfaces, or the PZT layer itself. We observed changing ferroelectric properties, especially remnant polarization $P_{r}$, with the number of measurement cycles as shown in Fig. 4.5 a). The coercive field $E_{c}$ shows no clear trend, therefore not plotted. Before comparing samples, first we explain the evolution of the remnant polarization. In all samples the remnant polarization $P_{r}$ increases first, reaches a maximum between $10^{2}$ and $10^{3}$ cycles and then decreases. Only sample C1 shows a different behavior with sharp decrease after 158 cycles due to electrical breakdown of the sample. The sample breakdown is due to the longer storage of the sample in air (3 months) compared to the other samples that were measured shortly after the fabrication. It is known that air humidity causes degradation of the Pt top electrodes on PZT films, leading to an earlier electrical breakdown [59]. Nevertheless, up to the breakdown the sample behavior is similar to samples A3 and A4 as exactly expected from Fig. 4.4.

To investigate the changes of the ferroelectric properties with cycling in more detail, the sample that shows the most apparent evolution, sample A1, is used as an example. The P-E and I-E loops are shown in Fig. 4.5 b) and Fig. 4.5 c) for three different cycling states. The initial loop before cycling is applied, the loop at the highest remnant polarization which occurs after $1.58 \times 10^{2}$ cycles, and the loop after the maximum number of $10^{4}$ cycles. The P-E loops show square like behavior indicating a good ferroelectric quality. We observe that all loops are slightly slanted and asymmetric around the horizontal axis. The slantiness of the loop decreases with increasing the number of cycles from 1 to $1.58 \times 10^{2}$, and thus the remnant polarization $P_{r}$ is increasing and reaching a maximum at $1.58 \times 10^{2}$ cycles. Afterwards, $P_{r}$ decreases continuously with increasing number of cycles. The asymmetry of the P-E loop is attributed to the difference in the work function of the electrodes that are from two different materials [23, 60], namely LNO and Pt. The changes due to cycling are even more clearly visible in the I-E loops. With increasing number of cycles, the broad initial peak slims down on both sides and a sharp peak develops. After $1.58 \times 10^{2}$ cycles the peak value starts to decrease but the shape is mostly preserved with a slight slimming. The negative switching peaks show a similar behavior with increasing number of cycles.

The increase of $P_{r}$ with cycling is known as wake-up effect and reported for some PZT films [61], and more commonly for $\mathrm{HfO}_{2}$ based ferroelectrics [62-66]. The wake-up for PZT is mostly observed for sol-gel films and attributed to pinning of domains in the films, which leads to internal bias fields and double peaks in the I-E loops [63]. With increased cycling of electric field the domains that are pinned due to defects like point defects, oxygen and/or lead vacancies or grain boundaries, 
reorient themselves $[63,67,68]$. The thus formed new domain structure is more stable and has a higher remnant polarization. The absence of two clearly visible switching peaks in our films, can be explained by the higher film quality due to PLD growth compared to sol-gel. Due to the better film quality we have less defects and thus the domains are only slightly pinned and do not form the clear double peak structure. Comparing the amount of wake-up between our samples, the differences can be correlated with the microstructure. For the columnar PZT samples, the grain size is smaller, and the domains are less clamped, as a result easier to switch and reach a stable domain configuration. The decrease of $P_{r}$ with further increasing number of cycles, i.e. fatigue, is well known for samples with Pt electrodes [69]. It is explained mainly by two models. The first model explains the polarization decrease with the migration and accumulation of oxygen vacancies at the film-electrode interface, causing domain pinning and charge screening. [70-75]. The second model considers the interfacial layer injecting electrons to the ferroelectric layer, leading to decomposition of the ferroelectric layer. [76-79] Existence of such an interfacial layer in the PLD PZT films has been recently proven using TEM measurements [80].

a)
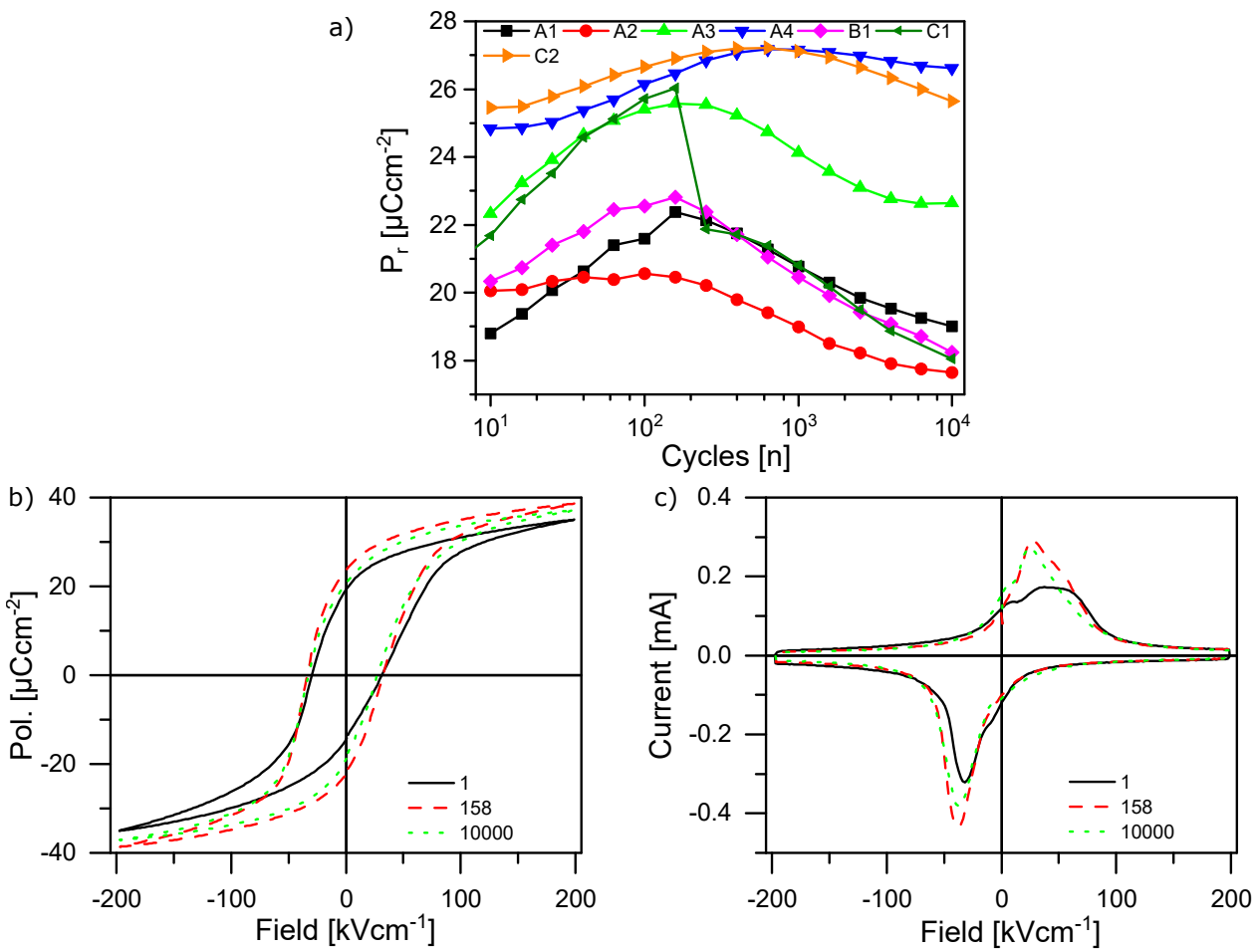

Figure 4.5: a) Effect of electric-field cycling on the remnant polarization Pr. for all samples with LNO/PZT. b) The P-E loop and c) the I-E loop for sample A1 with increasing number of electrical field cycling.

To make a fair comparison of the influence of the template layer to the ferroelec- 
tric properties, we compare the P-E and I-E loops of all samples in the woken-up state, where the $P_{r}$ is maximum in Fig. 4.6. As sample $\mathrm{C} 1$ broke before reaching the maximum it is excluded in Fig. 4.6. In the P-E loops the differences in the $P_{r}$ and maximum polarization $P_{\max }$ are visible, but changes in the coercive fields are less visible. For the samples with an RMS roughness above $20 \mathrm{~nm}$ and LNO peak-area ratio above 0.8 (A3, A4, C2), the slantiness of the P-E loops decreases, which results in an increase of $P_{r}$ and $P_{\max }$. The $P_{\max }$ measured for these samples are similar to the values reported on PZT films of the same thickness deposited on $100 \mathrm{~nm}$ thick $\mathrm{SrRuO}_{3}$ electrodes on $\mathrm{Si} / \mathrm{CNO}$ substrates [28] and PZT films of $750 \mathrm{~nm}$ thickness grown on $200 \mathrm{~nm}$ thick LNO on glass/CNO substrates [25], but smaller than values reported for films epitaxially grown on STO [28]. The samples with an RMS roughness below $20 \mathrm{~nm}$ and LNO peak-area ratio below 0.8 (A1, A2, B1) have a lower $P_{\max }$ and $P_{r}$ that are comparable with the structures deposited on $\mathrm{Si} / \mathrm{Pt} / \mathrm{CNO}$ substrates [28]. The samples with high roughness $(>20 \mathrm{~nm})$ have on average 1.2 times higher remnant polarization than the samples with low roughness, underlining the influence of the microstructure on the ferroelectric properties. In the I-E loops, the positive switching peaks are broader than the negative switching peaks for all samples due to the difference of the top and bottom electrode materials. Literature attributes wider switching peaks to larger variations in the grain sizes [81]. This literature finding suggests that the grain size variation is largest for the densest PZT films. Our films are consistent with this literature suggestion, considering that the grains in our dense PZT films can laterally grow as large as the few-micron-sized CNO nanosheets with a wide size distribution [53]. On the other hand, for the columnar films the column diameters are similar (between $100 \mathrm{~nm}$ to $150 \mathrm{~nm}$ ) and about a factor 10 smaller than a micrometer. The grain size distribution is not dependent on the size of the nanosheet anymore. Correspondingly, these samples show sharper switching peaks.

Within this study, we observe a clear correlation between film microstructure and remnant polarization $P_{r}$. The maximum polarization however does not clearly corollate with the film microstructure, as its value is not increasing continuously with increasing roughness. Thus, for energy storage devices, it is important to take the effect of the microstructure on the ferroelectric properties into account, when aiming to improve the energy storage capacities. The increase of the ferroelectric properties with increased roughness can be a clear indication of an increase in the piezoelectric properties through electrostriction [82]. The longitudinal piezoelectric coefficient $d_{33}$ is proportional to $P^{2}$, as such $d_{33}$ is expected to be higher for the rougher films with columnar microstructure. The ability to control the film microstructure using the template layer, from a dense smooth PZT film to a rough columnar PZT film, is interesting for actuators, as this allows control of the preferred actuation mode between $d_{31}$ mode with dense films or $d_{33}$ mode with columnar films. 

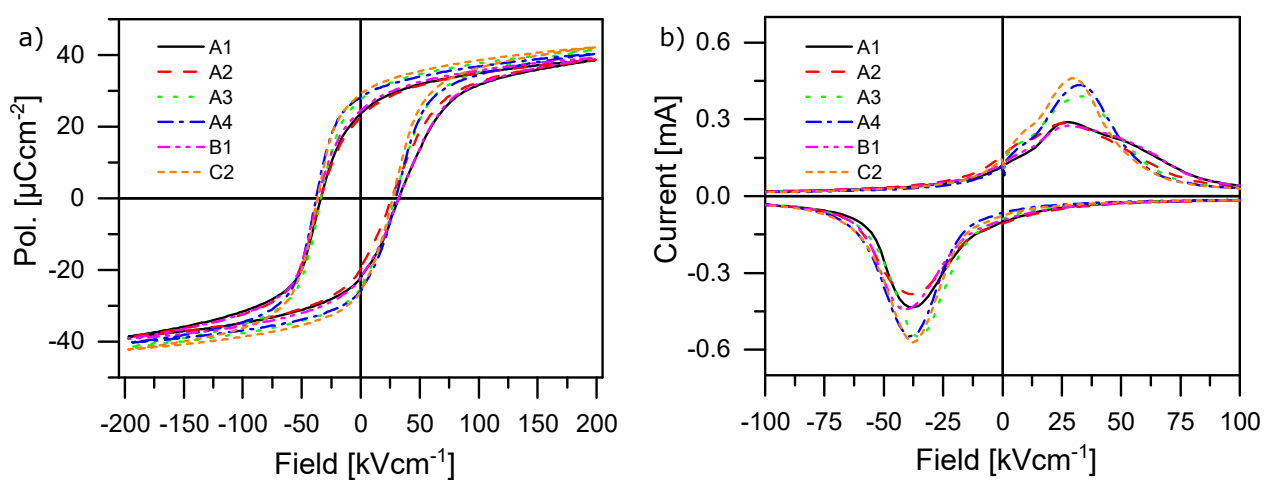

Figure 4.6: a) P-E and b) I-E loops at woken-up state where maximum $P_{r}$ is observed for the sample sets A, B and C. For better visibility only the data between $\pm 100 \mathrm{kV} \mathrm{cm}^{-1}$ is shown for the I-E loops. The difference between the samples outside this range was negligibly small.

\subsection{Conclusion}

We demonstrated the influence of the deposition pressure and the thickness of the LNO template layer on the microstructure and the ferroelectric properties of the subsequently grown PZT film. A higher oxygen pressure and a higher thickness of the LNO template was found to lead to a higher roughness and columnar growth of the PZT film. We attribute the change of the growth from 2D layer-by-layer growth to 3D island growth, to the increased roughness of the LNO layer in order to relax the epitaxial strain. The ferroelectric properties of the PZT films are changed for the films with columnar microstructure. This increase is explained by the reduced lateral grain size of the columnar PZT films. The average lateral grain size is much smaller and determined by the column width in columnar films, in contrast to the grain sizes that can reach the lateral size of a micrometer-sized nanosheet in dense films. The columnar microstructure leads to a smaller and more even grain size distribution and thus to changed ferroelectric properties.

\section{Acknowledgments}

This work is part of the research programme "Smart Multilayer Interactive Optics for Lithography at Extreme UV wavelengths (SMILE)", with contract number 10448 and it is financially supported by the Dutch Research Council (Nederlandse Organisatie voor Wetenschappelijk Onderzoek, NWO) and Carl Zeiss SMT. The authors thank the Inorganic Materials Science (IMS) Group of the University of Twente for supplying the self-made PZT target and especially Dr. Huiyu Yuan of the IMS group for the nanosheet deposition and Mr. Mark Smithers for performing the HRSEM experiment. 


\section{References}

[1] V. Nagarajan, A. Stanishevsky, L. Chen, T. Zhao, B.-T. Liu, J. Melngailis, A. L. Roytburd, R. Ramesh, J. Finder, Z. Yu, R. Droopad, and K. Eisenbeiser, Realizing intrinsic piezoresponse in epitaxial submicron lead zirconate titanate capacitors on Si, Applied Physics Letters 81, 4215 (2002).

[2] S. Trolier-McKinstry and P. Muralt, Thin Film Piezoelectrics for MEMS, Journal of Electroceramics 12, 7 (2004).

[3] N. Setter, D. Damjanovic, L. Eng, G. Fox, S. Gevorgian, S. Hong, A. Kingon, H. Kohlstedt, N. Y. Park, G. B. Stephenson, I. Stolitchnov, A. K. Tagantsev, D. V. Taylor, T. Yamada, and S. Streiffer, Ferroelectric thin films: Review of materials, properties, and applications, Journal of Applied Physics 100, 051606 (2006).

[4] C.-B. Eom and S. Trolier-McKinstry, Thin-film piezoelectric MEMS, MRS Bulletin 37, 1007 (2012).

[5] G. L. Smith, J. S. Pulskamp, L. M. Sanchez, D. M. Potrepka, R. M. Proie, T. G. Ivanov, R. Q. Rudy, W. D. Nothwang, S. S. Bedair, C. D. Meyer, and R. G. Polcawich, PZT-Based Piezoelectric MEMS Technology, Journal of the American Ceramic Society 95, 1777 (2012).

[6] M. Bayraktar, W. A. Wessels, C. J. Lee, F. A. van Goor, G. Koster, G. Rijnders, and F. Bijkerk, Active multilayer mirrors for reflectance tuning at extreme ultraviolet (EUV) wavelengths, Journal of Physics D: Applied Physics 45, 494001 (2012).

[7] M. Bayraktar, A. Chopra, G. Rijnders, K. Boller, and F. Bijkerk, Wavefront correction in the extreme ultraviolet wavelength range using piezoelectric thin films, Optics Express 22, 30623 (2014).

[8] M. Nematollahi, P. Lucke, M. Bayraktar, A. Yakshin, G. Rijnders, and F. Bijkerk, Nanoscale piezoelectric surface modulation for adaptive extreme ultraviolet and soft $x$-ray optics, Optics Letters 44, 5104 (2019).

[9] B. Jaffe, W. R. Cook, and H. Jaffe, Chapter 7 - Solid Solutions of Pb(Ti, Zr, $\mathrm{Sn}$, Hf) $\mathrm{O}_{3}$, in Piezoelectric Ceramics, edited by B. Jaffe, W. R. Cook, and H. Jaffe (Academic Press, 1971) pp. 135-183.

[10] M. J. Haun, E. Furman, S. J. Jang, and L. E. Cross, Thermodynamic theory of the lead zirconate-titanate solid solution system, part V: Theoretical calculations, Ferroelectrics 99, 63 (1989).

[11] R. Guo, L. E. Cross, S. E. Park, B. Noheda, D. E. Cox, and G. Shirane, Origin of the High Piezoelectric Response in $\mathrm{PbZr}_{1-x} \mathrm{Ti}_{x} \mathrm{O}_{3}$, Physical Review Letters 84, 5423 (2000).

[12] D. Damjanovic, A morphotropic phase boundary system based on polarization rotation and polarization extension, Applied Physics Letters 97, 062906 (2010). 
[13] X.-h. Du, U. Belegundu, and K. Uchino, Crystal Orientation Dependence of Piezoelectric Properties in Lead Zirconate Titanate: Theoretical Expectation for Thin Films, Japanese Journal of Applied Physics 36, 5580 (1997).

[14] J. Ouyang, S. Y. Yang, L. Chen, R. Ramesh, and A. L. Roytburd, Orientation dependence of the converse piezoelectric constantsfor epitaxial single domain ferroelectric films, Applied Physics Letters 85, 278 (2004).

[15] C. J. Kim, D. S. Yoon, J. S. Lee, C. G. Choi, W. J. Lee, and K. No, Electrical characteristics of (100), (111), and randomly aligned lead zirconate titanate thin films, Journal of Applied Physics 76, 7478 (1994).

[16] X.-h. Du, J. Zheng, U. Belegundu, and K. Uchino, Crystal orientation dependence of piezoelectric properties of lead zirconate titanate near the morphotropic phase boundary, Applied Physics Letters 72, 2421 (1998).

[17] P. Muralt, Ferroelectric thin films for micro-sensors and actuators: a review, Journal of Micromechanics and Microengineering 10, 136 (2000).

[18] D. Bao, R. Scholz, M. Alexe, and D. Hesse, Growth, microstructure, and ferroelectric properties of $\mathrm{Pb}\left(\mathrm{Zr}_{0.4} \mathrm{Ti}_{0.6}\right) \mathrm{O}_{3} / \mathrm{PbZrO}_{3}$ superlattices prepared on $\mathrm{SrTiO}_{3}$ (100) substrates by pulsed laser deposition, Journal of Applied Physics 101, 054118 (2007).

[19] D. K. Fork, D. B. Fenner, G. A. N. Connell, J. M. Phillips, and T. H. Geballe, Epitaxial yttria-stabilized zirconia on hydrogen-terminated Si by pulsed laser deposition, Applied Physics Letters 57, 1137 (1990).

[20] A. Kumar, M. R. Alam, A. Mangiaracina, and M. Shamsuzzoha, Synthesis of the PZT films deposited on Pt-coated (100) Si substrates for nonvolatile memory applications, Journal of Electronic Materials 26, 1331 (1997).

[21] S. J. Wang, C. K. Ong, L. P. You, and S. Y. Xu, Epitaxial growth of yittriastabilized zirconia oxide thin film on natively oxidized silicon wafer without an amorphous layer, Semiconductor Science and Technology 15, 836 (2000).

[22] M. Dekkers, M. D. Nguyen, R. Steenwelle, P. M. t. Riele, D. H. A. Blank, and G. Rijnders, Ferroelectric properties of epitaxial $\mathrm{Pb}(\mathrm{Zr}, \mathrm{Ti}) \mathrm{O}_{3}$ thin films on silicon by control of crystal orientation, Applied Physics Letters 95, 012902 (2009).

[23] S. H. Baek, J. Park, D. M. Kim, V. A. Aksyuk, R. R. Das, S. D. Bu, D. A. Felker, J. Lettieri, V. Vaithyanathan, S. S. N. Bharadwaja, N. Bassiri-Gharb, Y. B. Chen, H. P. Sun, C. M. Folkman, H. W. Jang, D. J. Kreft, S. K. Streiffer, R. Ramesh, X. Q. Pan, S. Trolier-McKinstry, D. G. Schlom, M. S. Rzchowski, R. H. Blick, and C. B. Eom, Giant Piezoelectricity on Si for Hyperactive $M E M S$, Science 334, 958 (2011).

[24] K. Kikuta, K. Noda, S. Okumura, T. Yamaguchi, and S.-i. Hirano, Orientation control of perovskite thin films on glass substrates by the application of 
a seed layer prepared from oxide nanosheets, Journal of Sol-Gel Science and Technology 42, 381 (2007).

[25] M. Bayraktar, A. Chopra, F. Bijkerk, and G. Rijnders, Nanosheet controlled epitaxial growth of $\mathrm{PbZr}_{0.52} \mathrm{Ti}_{0.48} \mathrm{O}_{3}$ thin films on glass substrates, Applied Physics Letters 105, 132904 (2014).

[26] Y. Minemura, Y. Kondoh, H. Funakubo, and H. Uchida, One-Axis-Oriented Crystal Growth of Lead Zirconate Titanate Thin Films on Metal Substrates Using Perovskite-Type Oxide Nanosheet Layer, Key Engineering Materials 582, 15 (2014).

[27] A. Chopra, M. Bayraktar, F. Bijkerk, and G. Rijnders, Controlled growth of PbZr0.52Ti0.4803 using nanosheet coated Si (001), Thin Solid Films 589, 13 (2015).

[28] M. D. Nguyen, H. Yuan, E. P. Houwman, M. Dekkers, G. Koster, J. E. ten Elshof, and G. Rijnders, Highly Oriented Growth of Piezoelectric Thin Films on Silicon Using Two-Dimensional Nanosheets as Growth Template Layer, ACS Applied Materials \& Interfaces 8, 31120 (2016).

[29] H. Uchida, D. Ichinose, T. Shiraishi, H. Shima, T. Kiguchi, A. Akama, K. Nishida, T. J. Konno, and H. Funakubo, Polarization switching behavior of one-axis-oriented lead zirconate titanate films fabricated on metal oxide nanosheet layer, Japanese Journal of Applied Physics 56, 10PF10 (2017).

[30] N. Izyumskaya, Y. I. Alivov, S. J. Cho, H. Morkoç, H. Lee, and Y. S. Kang, Processing, structure, properties, and applications of PZT thin films, Critical Reviews in Solid State and Materials Sciences 32, 111 (2007).

[31] E. P. Houwman, M. D. Nguyen, M. Dekkers, and G. Rijnders, Intrinsic stability of ferroelectric and piezoelectric properties of epitaxial $\mathrm{PbZr}_{0.45} \mathrm{Ti}_{0.55} \mathrm{O}_{3}$ thin films on silicon in relation to grain tilt, Science and Technology of Advanced Materials 14, 045006 (2013).

[32] C. S. Ganpule, A. Stanishevsky, S. Aggarwal, J. Melngailis, E. Williams, R. Ramesh, V. Joshi, and C. P. d. Araujo, Scaling of ferroelectric and piezoelectric properties in $\mathrm{Pt} / \mathrm{SrBi}_{2} \mathrm{Ta}_{2} \mathrm{O}_{9} / \mathrm{Pt}$ thin films, Applied Physics Letters 75, 3874 (1999).

[33] S. Bühlmann, B. Dwir, J. Baborowski, and P. Muralt, Size effect in mesoscopic epitaxial ferroelectric structures: Increase of piezoelectric response with decreasing feature size, Applied Physics Letters 80, 3195 (2002).

[34] J.-H. Li, L. Chen, V. Nagarajan, R. Ramesh, and A. L. Roytburd, Finite element modeling of piezoresponse in nanostructured ferroelectric films, Applied Physics Letters 84, 2626 (2004).

[35] A. Chopra, M. Bayraktar, M. Nijland, J. E. ten Elshof, F. Bijkerk, and G. Rijnders, Tuning of large piezoelectric response in nanosheet-buffered lead zirconate titanate films on glass substrates, Scientific Reports 7, 251 (2017). 
[36] M. D. Nguyen, E. P. Houwman, M. Dekkers, and G. Rijnders, Strongly Enhanced Piezoelectric Response in Lead Zirconate Titanate Films with Vertically Aligned Columnar Grains, ACS Applied Materials \& Interfaces 9, 9849 (2017).

[37] M. D. Nguyen, E. P. Houwman, and G. Rijnders, Large piezoelectric strain with ultra-low strain hysteresis in highly c-axis oriented $\mathrm{Pb}\left(Z_{0.52} \mathrm{Ti}_{0.48}\right) \mathrm{O}_{3}$ films with columnar growth on amorphous glass substrates, Scientific Reports 7 (2017), 10.1038/s41598-017-13425-w.

[38] M. D. Nguyen, E. P. Houwman, H. Yuan, B. J. Wylie-Van Eerd, M. Dekkers, G. Koster, J. E. Ten Elshof, and G. Rijnders, Controlling Piezoelectric Responses in $\mathrm{Pb}\left(\mathrm{Zr}_{0.52} \mathrm{Ti}_{0.48}\right) \mathrm{O}_{3}$ Films through Deposition Conditions and Nanosheet Buffer Layers on Glass, ACS Applied Materials \& Interfaces 9, 35947 (2017).

[39] X. G. Tang, H. L. W. Chan, A. L. Ding, and Q. R. Yin, Processing effects on the microstructure and ferroelectric properties of $\mathrm{Pb}(\mathrm{Zr}, \mathrm{Ti}) \mathrm{O}_{3}$ thin films prepared by sol-gel process, Surface and Coatings Technology 161, 169 (2002).

[40] F. Chu, F. Xu, J. Shepard, and S. Trolier-McKinstry, Thickness Dependence of the Electrical Properties of Sol-Gel Derived Lead Zirconate Titanate Thin Films with (111) and (100) Texture, MRS Proceedings 493, 409 (1997).

[41] R. Bouregba, G. Poullain, B. Vilquin, and H. Murray, Epitaxial PZT thin films on TiOx covered Pt/MgO substrate by RF magnetron sputtering, Ferroelectrics 256, 47 (2001).

[42] I. Kanno, H. Kotera, K. Wasa, T. Matsunaga, T. Kamada, and R. Takayama, Crystallographic characterization of epitaxial $\mathrm{Pb}(\mathrm{Zr}, \mathrm{Ti}) \mathrm{O}_{3}$ films with different $\mathrm{Zr} /$ Ti ratio grown by radio-frequency-magnetron sputtering, Journal of Applied Physics 93, 4091 (2003).

[43] H. Jacobsen, K. Prume, B. Wagner, K. Ortner, and T. Jung, High-rate sputtering of thick PZT thin films for MEMS, Journal of Electroceramics 25, 198 (2010).

[44] O. Nordseth, C. C. You, E. Folven, S. Gariglio, A. Sambri, J.-M. Triscone, J. W. Reiner, C. H. Ahn, T. Tybell, and J. K. Grepstad, Growth and characterization of $(\mathrm{Pb}, \mathrm{La})(\mathrm{Zr}, \mathrm{Ti}) \mathrm{O}_{3}$ thin film epilayers on $\mathrm{SrTiO}_{3}$-buffered $\mathrm{Si}(001)$, Thin Solid Films 518, 5471 (2010).

[45] S. Yoshida, H. Hanzawa, K. Wasa, M. Esashi, and S. Tanaka, Highly c-axisoriented monocrystalline $\mathrm{Pb}(\mathrm{Zr}, \mathrm{Ti}) \mathrm{O}_{3}$ thin films on si wafer prepared by fast cooling immediately after sputter deposition, IEEE Transactions on Sonics and Ultrasonics 61, 1552 (2014).

[46] H. Palneedi, M. Peddigari, G.-T. Hwang, D.-Y. Jeong, and J. Ryu, HighPerformance Dielectric Ceramic Films for Energy Storage Capacitors: Progress and Outlook, Advanced Functional Materials 28, 1803665 (2018). 
[47] H. Liu, J. Zhong, C. Lee, S.-W. Lee, and L. Lin, A comprehensive review on piezoelectric energy harvesting technology: Materials, mechanisms, and applications, Applied Physics Reviews 5, 041306 (2018).

[48] M. R. Sarker, S. Julai, M. F. M. Sabri, S. M. Said, M. M. Islam, and M. Tahir, Review of piezoelectric energy harvesting system and application of optimization techniques to enhance the performance of the harvesting system, Sensors and Actuators A: Physical 300, 111634 (2019).

[49] P. Muralt, R. G. Polcawich, and S. Trolier-McKinstry, Piezoelectric Thin Films for Sensors, Actuators, and Energy Harvesting, MRS Bulletin 34, 658 (2009).

[50] H. Yuan, R. Lubbers, R. Besselink, M. Nijland, and J. E. ten Elshof, Improved Langmuir-Blodgett Titanate Films via in Situ Exfoliation Study and Optimization of Deposition Parameters, ACS Applied Materials \& Interfaces 6, 8567 (2014).

[51] L. Guan, D. Zhang, X. Li, and Z. Li, Role of pulse repetition rate in film growth of pulsed laser deposition, Nuclear Instruments \& Methods in Physics Research, Section B: Beam Interactions with Materials and Atoms 266, 57 (2008).

[52] Y.-Z. Chen, T.-H. Liu, C.-Y. Chen, C.-H. Liu, S.-Y. Chen, W.-W. Wu, Z. L. Wang, J.-H. He, Y.-H. Chu, and Y.-L. Chueh, Taper $\mathrm{PbZr}_{0.2} \mathrm{Ti}_{0.8} \mathrm{O}_{3}$ Nanowire Arrays: From Controlled Growth by Pulsed Laser Deposition to Piezopotential Measurements, ACS Nano 6, 2826 (2012).

[53] H. Yuan, M. Nguyen, T. Hammer, G. Koster, G. Rijnders, and J. E. ten Elshof, Synthesis of $\mathrm{KCa}_{2} \mathrm{Nb}_{3} \mathrm{O}_{10}$ Crystals with Varying Grain Sizes and Their Nanosheet Monolayer Films As Seed Layers for PiezoMEMS Applications, ACS Applied Materials \& Interfaces 7, 27473 (2015).

[54] L. Qiao and X. F. Bi, Strain state, microstructure and electrical transport properties of $\mathrm{LaNiO}_{3}$ films grown on Si substrates, Journal of Physics D: Applied Physics 41, 195407 (2008).

[55] H. Wei, M. Jenderka, M. Grundmann, and M. Lorenz, LaNiO 3 films with tunable out-of-plane lattice parameter and their strain-related electrical properties, Physica Status Solidi A: Applied Research 212, 1925 (2015).

[56] G. Springholz, N. Frank, and G. Bauer, The origin of surface roughening in lattice-mismatched Frank van der Merwe type heteroepitaxy, Thin Solid Films 267, 15 (1995).

[57] M. Volmer and A. Weber, Keimbildung in übersättigten Gebilden, Zeitschrift für Physikalische Chemie 119, 277 (1926).

[58] I. Stranski and L. Krastanov, Theory of orientation separation of ionic crystals, Sitzungsberichte Akademische Wissenschaften Wien 146, 797 (1938). 
[59] J. Wang, C. Salm, E. Houwman, M. Nguyen, and J. Schmitz, Humidity and polarity influence on MIM PZT capacitor degradation and breakdown, in Proceedings of the 2016 IEEE International Integrated Reliability Workshop, IIRW 2016 (2016) pp. 65-68.

[60] J. Lee, C. H. Choi, B. H. Park, T. W. Noh, and J. K. Lee, Built-in voltages and asymmetric polarization switching in $\mathrm{Pb}\left(\mathrm{Zr}\right.$, Ti) $\mathrm{O}_{3}$ thin film capacitors, Applied Physics Letters 72, 3380 (1998).

[61] F. C. Kartawidjaja, C. H. Sim, and J. Wang, Ferroelectric and dielectric behavior of heterolayered PZT thin films, Journal of Applied Physics 102 (2007), $10.1063 / 1.2822472$.

[62] T. Schenk, M. Hoffmann, J. Ocker, M. Pešic̀, T. Mikolajick, and U. Schroeder, Complex Internal Bias Fields in Ferroelectric Hafnium Oxide, ACS Applied Materials \& Interfaces 7, 20224 (2015).

[63] F. P. G. Fengler, M. Pešic̀, S. Starschich, T. Schneller, C. Künneth, U. Böttger, H. Mulaosmanovic, T. Schenk, M. H. Park, R. Nigon, P. Muralt, T. Mikolajick, and U. Schroeder, Domain Pinning: Comparison of Hafnia and PZT Based Ferroelectrics, Advanced Electronic Materials 3, 1600505 (2017).

[64] H. J. Kim, M. H. Park, Y. J. Kim, Y. H. Lee, T. Moon, K. D. Kim, S. D. Hyun, and C. S. Hwang, A study on the wake-up effect of ferroelectric $\mathrm{Hf}_{0.5} \mathrm{Zr}_{0.5} \mathrm{O}_{2}$ films by pulse-switching measurement, Nanoscale 8, 1383 (2016).

[65] L. Zhang, J. Chen, J. Zhang, H. Wang, L. Xu, and X. Xing, Domainreorientation-induced polarization wake-up of $\mathrm{PbTiO}_{3}$ based ferroelectric thin films, Ceramics International 42, 19212 (2016).

[66] D. Zhou, J. Xu, Q. Li, Y. Guan, F. Cao, X. Dong, J. Müller, T. Schenk, and U. Schroeder, Wake-up effects in Si-doped hafnium oxide ferroelectric thin films, Applied Physics Letters 103, 192904 (2013).

[67] F. P. G. Fengler, M. Pešic̀, S. Starschich, T. Schneller, U. Böttger, T. Schenk, M. H. Park, T. Mikolajick, and U. Schroeder, Comparison of hafnia and PZT based ferroelectrics for future non-volatile FRAM applications, in 2016 46th European Solid-State Device Research Conference (ESSDERC), pp. 369-372.

[68] M. Kohli, P. Muralt, and N. Setter, Removal of $90^{\circ}$ domain pinning in (100)$\mathrm{Pb}\left(Z_{0.15} \mathrm{Ti}_{0.85}\right) \mathrm{O}_{3}$ thin films by pulsed operation, Applied Physics Letters 72, 3217 (1998).

[69] A. K. Tagantsev, I. Stolichnov, E. L. Colla, and N. Setter, Polarization fatigue in ferroelectric films: Basic experimental findings, phenomenological scenarios, and microscopic features, Journal of Applied Physics 90, 1387 (2001).

[70] V. C. Lo, Modeling the role of oxygen vacancy on ferroelectric properties in thin films, Journal of Applied Physics 92, 6778 (2002). 
[71] H. Z. Jin and J. Zhu, Size effect and fatigue mechanism in ferroelectric thin films, Journal of Applied Physics 92, 4594 (2002).

[72] S. M. Yang, T. H. Kim, J.-G. Yoon, and T. W. Noh, Nanoscale Observation of Time-Dependent Domain Wall Pinning as the Origin of Polarization Fatigue, Advanced Functional Materials 22, 2310 (2012).

[73] J. F. Scott and M. Dawber, Oxygen-vacancy ordering as a fatigue mechanism in perovskite ferroelectrics, Applied Physics Letters 76, 3801 (2000).

[74] M. Dawber and J. F. Scott, A model for fatigue in ferroelectric perovskite thin films, Applied Physics Letters 76, 1060 (2000).

[75] A. Chopra, M. Bayraktar, M. Nijland, J. E. t. Elshof, F. Bijkerk, and G. Rijnders, Polarization recovery in lead zirconate titanate thin films deposited on nanosheets-buffered Si (001), AIP Advances 6, 125209 (2016).

[76] X. J. Lou, M. Zhang, S. A. T. Redfern, and J. F. Scott, Fatigue as a local phase decomposition: A switching-induced charge-injection model, Physical Review B: Condensed Matter and Materials Physics 75, 224104 (2007).

[77] M. Wu, W. Li, J. Li, S. Wang, Y. Li, B. Peng, H. Huang, and X. Lou, Fatigue mechanism verified using photovoltaic properties of $\mathrm{Pb}\left(\mathrm{Zr}_{0.52} \mathrm{Ti}_{0.48}\right) \mathrm{O}_{3}$ thin films, Applied Physics Letters 110, 133903 (2017).

[78] X. J. Lou, Why do antiferroelectrics show higher fatigue resistance than ferroelectrics under bipolar electrical cycling? Applied Physics Letters 94, 072901 (2009).

[79] X. J. Lou, M. Zhang, S. A. T. Redfern, and J. F. Scott, Local Phase Decomposition as a Cause of Polarization Fatigue in Ferroelectric Thin Films, Physical Review Letters 97, 177601 (2006).

[80] M. T. Do, N. Gauquelin, M. D. Nguyen, J. Wang, J. Verbeeck, F. Blom, G. Koster, E. P. Houwman, and G. Rijnders, Interfacial dielectric layer as an origin of polarization fatigue in ferroelectric capacitors, Scientific Reports 10, 7310 (2020).

[81] T. Schenk, E. Yurchuk, S. Mueller, U. Schroeder, S. Starschich, U. Böttger, and T. Mikolajick, About the deformation of ferroelectric hystereses, Applied Physics Reviews 1 (2014), 10.1063/1.4902396.

[82] F. Li, L. Jin, Z. Xu, and S. Zhang, Electrostrictive effect in ferroelectrics: An alternative approach to improve piezoelectricity, Applied Physics Reviews 1, 011103 (2014). 


\section{5}

\section{Hysteresis, loss and nonlinearity in epitaxial $\mathrm{PbZr}_{0.55} \mathrm{Ti}_{0.45} \mathrm{O}_{3}$ films: $\mathrm{A}$ polarization rotation model}

The phenomena of hysteresis, ferroelectric loss and nonlinearity have been investigated for the strain and polarization of a monoclinic, epitaxial $\mathrm{Pb}(\mathrm{Zr}, \mathrm{Ti}) \mathrm{O}_{3}$ film over the $70 \mathrm{~Hz}$ to $5 \mathrm{kHz}$ frequency range at sub-coercive excitation fields and zero electrical bias. For the strain, a linear hysteretic behavior was found, whereas the polarization shows a strongly nonlinear hysteretic behavior. In contrast to polycrystalline structures (for instance in ceramics or chemical solution deposited thin films), the commonly referred Rayleigh model cannot explain the observed behavior. We present a new model, based on the rotation of the polarization vector within the monoclinic unit cell under an applied electric field, with the viscous interaction of domains accompanying the unit cell deformation. The model explains the amplitude and frequency scaling of the strain, polarization and loss tangent as well as the observed higher harmonics of polarization in the measured epitaxial $\mathrm{Pb}(\mathrm{Zr}, \mathrm{Ti}) \mathrm{O}_{3}$ films. We conclude that the nonlinear response and the hysteretic loss originate from two separate physical processes. The nonlinear response is attributed to the nonlinear angular rotation of the polarization vector, whereas the hysteresis and ferroelectric loss are due to a viscous interaction of domains while the polarization vector is rotating. 


\subsection{Introduction}

Nowadays piezoelectric ceramics and thin films are widely applied in sensors, actuators, ferroelectric memories and energy harvesters [1-8]. Especially $\mathrm{PbZr}_{x} \mathrm{Ti}_{1-x} \mathrm{O}_{3}$ (PZT) is commonly used because of its remarkable piezoelectric properties both in ceramics and thin films [9-11]. One of the challenges in the application of piezoelectric materials is the hysteresis that manifests itself, for example, as positioning inaccuracy of actuators and energy loss in energy harvesting applications [12]. Therefore, an accurate description and identification of the origin of hysteresis and energy loss in piezoelectric materials has always been an important topic.

Already early on, the interaction of domains was identified as a possible source of sub-coercive field hysteresis and was associated with dielectric and piezoelectric losses. The observed dielectric losses have been described by viscous motion of the domain walls (DWs) and in this way Arlt and Dederichs related the hysteretic piezoelectric response to the vibration of $90^{\circ} \mathrm{DWs}$ under an externally applied oscillating field [13-17]. However, with this model it is not possible to explain the observed field amplitude dependence of the dielectric/piezoelectric loss. Damjanovic described the field amplitude dependent dielectric/piezoelectric loss behavior in PZT ceramics with an adaptation of the Rayleigh model that has often been used for the description of hysteresis in ferromagnets and later also in ferroelectrics [18-21]. Microscopically the nonlinear field response, of which hysteresis is considered to be a consequence, has been related to the stochastic interaction of DWs with defects, causing so-called Barkhausen jumps of the DWs from a local energy minimum to the next [22, 23]. This model allows describing the effect of the amplitude and frequency of the applied electric field, to our best knowledge, only for polycrystalline ceramics and chemical solution deposited (CSD) thin films [24-27]. However we note that the Rayleigh model is based on a stochastic effect that does not take into account the crystal symmetry of the material. This works well for polycrystalline material, but one expects hysteresis and especially the nonlinearity to behave differently for different crystallinities, as the functional properties of materials with different crystal symmetries and crystal orientations, respond differently to the applied electric field [28]. Epitaxial thin films are therefore excellent model systems to investigate the relation between crystal symmetry and hysteresis as they resemble the behavior of perfect single crystal systems with low levels of defects. In addition, such investigation may render practical benefits since many future high-performance applications are expected to be based on epitaxial films because of their better performance. To our knowledge there are no studies available on the frequency and amplitude dependency of hysteresis, loss and nonlinearity in epitaxial PZT thin films.

We investigated the hysteresis, loss and nonlinearity of poled, epitaxial, (001)oriented, monoclinic PZT films at zero bias field for excitation electric field amplitudes up to half of the coercive field and in the frequency range of $70 \mathrm{~Hz}$ to $5 \mathrm{kHz}$. To explain the observed strain, polarization and dielectric/piezoelectric loss behavior, we propose a new model by considering the oscillatory rotation of the 
polarization vector in the monoclinic unit cell in response to an oscillating applied external electric field.

\subsection{Model development using experimental constraints}

Generally, hysteresis in ferro- and piezo-electric materials has been modelled with the Rayleigh model, which originates from the description of hysteresis in magnetic systems [18-21]. The Rayleigh model can be shown to be a limiting case of the Preisach model, which provides a statistical description of the transitions between local energy minima in an energy landscape under influence of an oscillatory field [29-31]. In the case of ferroelectrics, one assumes that DWs are locally trapped by defects and jump to new defect sites under the influence of the applied field, which is causing the hysteresis. In the Supporting Information Section A.1 we summarize the Rayleigh model and several extensions of it, that have been suggested over time. The main features of these models are:

a) Nonlinear response and hysteresis are described by the same hysteresis parameter (generally denoted by $\alpha$ ), therefore the magnitude of these phenomena are coupled [21, 24, 25, 32-38].

b) A Fourier decomposition of the hysteresis response only contains odd harmonics [21, 24, 25, 32-38]. In one extension of the model, including $180^{\circ} \mathrm{DW}$ motion, an additional second harmonic arises, but no even order higher harmonics appear [3941].

c) The hysteresis loop is lenticularly shaped, which most clearly shows up in sharp endpoints of the loop. In some models a viscous loss term is added to the polarization response, reflecting viscous motion of the domain walls under influence of the applied field. This viscous term introduces rounding of the hysteresis loop that becomes elliptically shaped. The viscous loss term may in some cases be the only reason for hysteresis [30, 32].

d) In the pure Rayleigh model the hysteresis loss does not contain an explicit frequency dependence, unless the dielectric or piezoelectric coefficient and/or the hysteresis parameters are implicitly frequency dependent $[26,26]$. In the models containing a viscous loss term, the loss scales linearly with frequency, apart from further implicit frequency dependencies [30, 32].

e) In the Rayleigh model the hysteresis loop is traversed counterclockwise (CCW), as one would expect for a lossy hysteresis process.

For the discussion here it is important to note that hysteresis models, as presented so far in literature, do not contain any relation with the crystal structure and crystal orientation of the materials to which the models are applied. These models may fairly well describe the hysteretic behavior of polycrystalline samples and probably also of many CSD derived thin films, however one would expect to see crystal symmetry dependence of the ferroelectric response in single crystals and epitaxial thin films.

The hysteresis measurements on our monoclinic, epitaxial PZT film show many deviations from the predictions of the Rayleigh model and its derivatives. Notably, 
we see only the fundamental harmonic in the strain response, whereas all even and odd harmonics up to the $5^{\text {th }}$ order appear in the polarization response. Furthermore, the hysteresis loops have an elliptical shape and the loss tangent is found to scale linearly with frequency. For these reasons we conclude that none of the abovementioned models can describe our measurement data and we propose a new model, based on the crystal symmetry, that describes the main observations.

\subsubsection{Polarization rotation model}

Here we describe a new model that can explain the observed hysteresis and nonlinearity in terms of the unit cell strain and polarization response induced by the applied electric field in (001)-oriented ferroelectric films with a monoclinic crystal symmetry. This also makes the model applicable to films with rhombohedral symmetry, that under the influence of an applied field change its symmetry to monoclinic as is observed for PZT and other materials. [42-45] The property we use here is that the (110)-plane is the symmetry plane in which the polarization vector rotates, i.e. that this plane contains the longest body diagonal of the unit cell such as in monoclinic unit cells. [46] Fig. 5.1 a) shows one of the eight possible configurations of such a unit cell, as one would expect to be present in approximately equal fractions in an as-grown epitaxial thin film, organized in polarization domains, separated by domain walls. The eight configurations correspond to unit cells in pseudocubic notation with the polarization vector in one of the eight possible $<111>$-like directions. For fully relaxed rhombohedral unit cells these would be the $<111>$ body diagonal directions. PZT films with top and bottom electrodes in the parallel plate configuration are considered to be poled in the downward direction (i.e. from top to bottom electrode) so that only the four configurations with the same downward oriented out-of-plane polarization component remain, which are organized in polarization domains separated by DWs. In this configuration, the polarization measurable across the film is,

$$
P=P_{S} \cos (\theta)
$$

where $P_{S}$ is the spontaneous polarization and $\theta$ is the polarization angle between the [001]-axis and the polarization vector. For the PZT films that will be explored here, the reference spontaneous polarization value in bulk rhombohedral PZT(55/45) composition is $P_{S}=50 \mathrm{\mu C} \mathrm{cm}^{-2}$. [47] For a fully relaxed rhombohedral unit cell with the polarization vector in the body diagonal, $\theta$ equals $\theta_{0}=54.7^{\circ}$ and hence $P$ equals $P_{r}=29 \mu \mathrm{C} \mathrm{cm}^{-2}$, the remanent polarization of the film [47]. In a film under compressive in-plane stress the polarization vector will rotate towards the out-ofplane direction so that the polarization angle of the film at zero field, $\theta_{0, f}$ decreases and the remanent polarization $P_{r, f}$ will increase. The stress leads to a deformation of the unit cell and breaking of the 3-fold symmetry and the film changes it crystal symmetry from rhombohedral to monoclinic. [45] Under an applied electric field in the [001]-direction, the polarization vector rotates following the electric field. For downward oriented fields (i.e. positive voltage on the top electrode with respect to a grounded bottom electrode) the polarization vector will rotate towards the out-of-plane [001]-axis, decreasing $\theta$, increasing $P$ and stretching the unit cell in 
[001]-direction by the piezoelectric effect. It is important to note that here, we only consider the oscillating electric field amplitudes below the coercive field value and assume that no polarization switching occurs. Additionally, we consider that the polarization extension is negligible in the considered sub-coercive electric field range ${ }^{1}$.
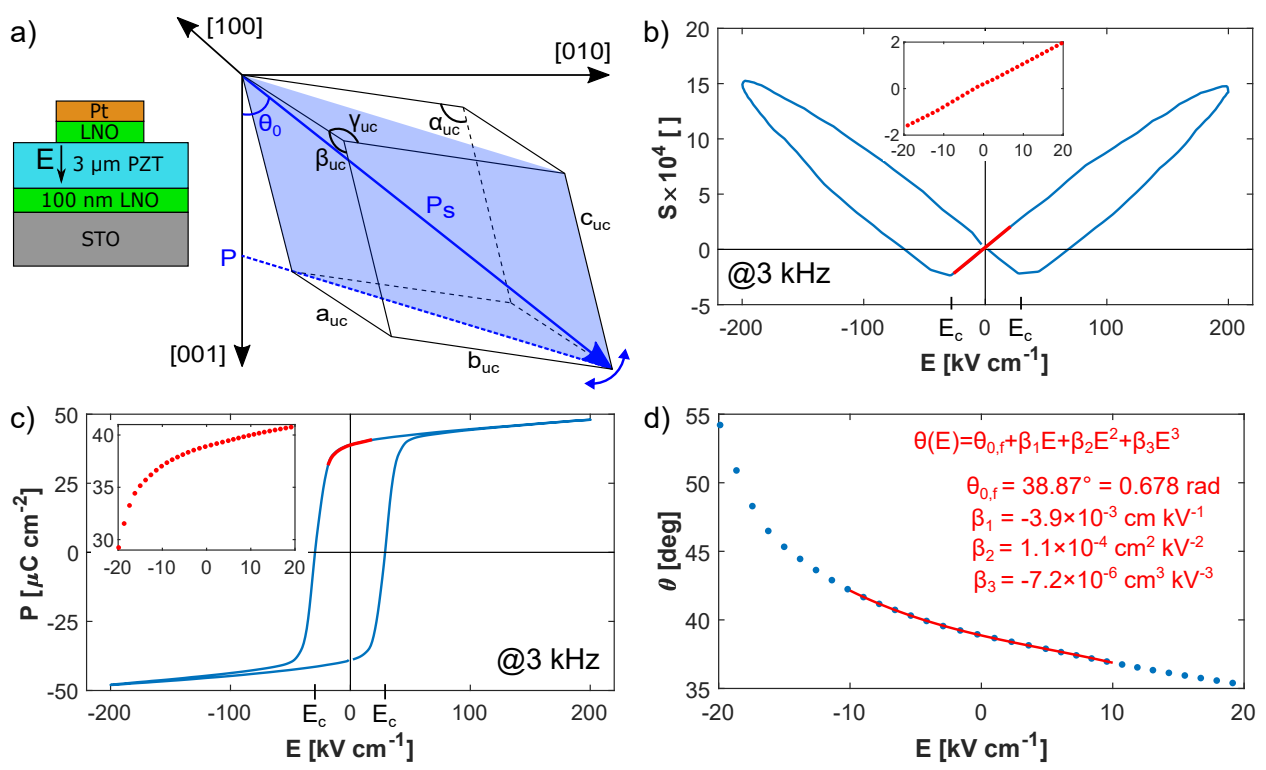

Figure 5.1: a) Schematic sample structure. Unstrained rhombohedral or monoclinic PZT unit cell with out-of-plane polarization component $P . P_{s}$ is the spontaneous polarization vector length and $\theta_{0}$ is the angle between the [001]-axis and the polarization vector. b) S-E and c) P-E loops for a large electric field amplitude, $E_{\max }=200 \mathrm{kV} \mathrm{cm}^{-1} \ll E_{c}$. The insets show the magnified view of the -20 to $20 \mathrm{kV} \mathrm{cm}^{-1}$ sub-coercive field range of the falling branches. d) Field dependence of the polarization angle $\theta$, calculated from the inset in c). The red line gives the fit of a third order polynomial over the -10 to $10 \mathrm{kV} \mathrm{cm}^{-1}$ field range as explained in the text. All the measurements are at $3 \mathrm{kHz}$.

\section{Strain response}

A typical strain-electric field (S-E) loop measured with a large electric field amplitude, far exceeding the coercive field, i.e. the global S-E loop, is shown in Fig. 5.1 b). The measured strain is in good approximation linear $\left(R^{2}=0.9987\right)$ with the applied field in the sub-coercive field range, as shown in the inset. In the first part of constructing a strain hysteresis model, we therefore take the strain to be linearly dependent and in-phase with the applied electric field $S_{\mathrm{ip}}(t)=d_{33, f} E(t)$ in the sub-coercive field range that is explored in the hysteresis experiments. As we work with films, the piezoelectric properties of the films are reduced compared to bulk

\footnotetext{
${ }^{1}$ In ref. [48, 49] it was shown, by Landau-Devonshire modelling of a clamped rhombohedral film on STO with PZT 60/40 composition, that the contribution to polarization change due to polarization rotation is a factor 3.2 larger than for polarization extension.
} 
properties due to clamping by the substrate. Here $d_{33, f}$ is the effective piezoelectric coefficient in the out-of-plane direction of the clamped film. In the Supporting Information Section A.2 we show that this linear dependence can be derived in good approximation from the Landau-Devonshire description of a single domain rhombohedral clamped film, at least for positive fields. In the Landau-Devonshire description the change of symmetry from rhombohedral to monoclinic due to the applied field is taken into account. [50] An expression for $d_{33, f}$ for a clamped film in terms of elastic compliances and electro-strictive parameters, determined from the Landau-Devonshire model, is also given. The elliptic strain hysteresis loops observed in our experiments suggest that the piezoelectric loss is dominated by a viscous loss mechanism, for example due to domain wall motion as commonly is assumed for piezoelectric materials [13, 15, 17]. In the second part of the strain model, a viscous and out-of-phase term that is proportional to the time derivative of the in-phase part can be written as $S_{\mathrm{oop}}(t)=\gamma_{S} \dot{S_{\mathrm{ip}}}(t)$ in which $\gamma_{S}$ is the viscosity coefficient. Total strain can be written as a summation of the in-phase and out-of-phase strain parts as $S(t)=S_{\mathrm{ip}}(t)+S_{\mathrm{oop}}(t)$. Hence, one arrives at a rather simple expression for the total strain response of the considered film to a small amplitude sinusoidal field, $E(t)=E_{0} \sin (\omega t)$, from which the hysteresis properties can be determined

$$
S(t)=d_{33, f} E(t)+\gamma_{S} d_{33, f} \dot{E}(t)=d_{33, f} E_{0} \sin (\omega t)+\gamma_{d} \omega E_{0} \cos (\omega t) .
$$

Here $\gamma_{d}$ is the multiplication of the viscosity coefficient $\gamma_{S}$ with the piezoelectric coefficient $d_{33, f}$. Because viscous processes cause a time lag of the unit cell expansion in response to increasing fields, one expects that $\gamma<0$. Note that Eq. 5.2 only describes the explicit excitation field amplitude and frequency dependence, but frequency dependent viscous interactions may lead to additional implicit frequency dependences of the parameters $d_{33, f}$ and $\gamma_{d}$. The nature of these viscous interactions is poorly understood and described in literature. We do not include such interactions in our model, but extract these dependencies from frequency dependent measurements. Eq. 5.2 predicts a hysteretic strain that is linearly dependent on field amplitude. In the simplest case, i.e. when $d_{33, f}$ and $\gamma_{d}$ are independent of frequency, the hysteresis is linearly dependent on the frequency and amplitude. The maximum loop opening, $\Delta S_{\text {open }}=|S(\omega t=\pi)-S(\omega t=0)|=\left|2 \gamma_{d} \omega E_{0}\right|$ scales linearly with $E_{0}$ and is, if $\gamma_{d}$ is not a function of frequency, also linearly with frequency. The strain loss, or the loss tangent, is obtained as the ratio of the viscous to non-viscous components of the strain:

$$
\tan \delta_{S}=\left|\gamma_{d} \omega / d_{33, f}\right|=\left|\gamma_{S} \omega\right|
$$

Interestingly, if $d_{33, f}$ and $\gamma_{d}$ are independent of amplitude and frequency, the loss tangent is independent of the amplitude and scales linearly with the frequency, which will be verified in the results section. In comparison the Rayleigh model predicts a dependency of the loss tangent on the amplitude of the electric field, see Supporting Information Eq. A.8, but on the other hand does not have an explicit frequency dependence. 


\section{Polarization response}

We assume that the polarization change is only due to polarization rotation and the effect of polarization vector extension is neglected $[48,49]$. For a positive field in the [001]-direction the polarization vector rotates towards the [001]-axis and for negative electric field towards the (001)-plane. The change of the polarization angle $\theta$ that is in-phase with the driving field can be described by $\theta_{\text {ip }}(E)=\theta_{0, f}+\Delta \theta(E)$, with $\theta_{0, f}$ the angle for a clamped film at zero field and $\Delta \theta(E)$ the small change of the angle with applied field. A typical global polarization-electric field (P-E) loop is shown in Fig. 5.1 c). The inset displays the part of the global loop in the field range -20 to $20 \mathrm{kV} \mathrm{cm}^{-1}$. Here we only consider the decreasing field branch, as the film is poled downward. During small amplitude field cycling in the -10 to $10 \mathrm{kV} \mathrm{cm}^{-1}$ range, i.e. well below the coercive-field so that no polarization switching occurs, the polarization tracks this branch back and forth.

Fig. $5.1 \mathrm{~d})$ gives the angle $\theta(E)$ calculated from the global P-E loop in Fig. $5.1 \mathrm{c}$ ) using Eq. 5.1. Fitting in the -10 to $10 \mathrm{kV} \mathrm{cm}^{-1}$ field range with a third order polynomial for $\Delta \theta(E)=\beta_{1} E+\beta_{2} E^{2}+\beta_{3} E^{3}$ in $\theta_{i p}(E)$ gives a very accurate description of the data $\left(R^{2}=0.9999\right)$. We note that a third order polynomial is the lowest order polynomial that can accurately describe the asymmetry of the measured $\theta(E)$ data with respect to the y-axis. In the following we will see that the nonlinear $\Delta \theta(E)$ relation accounts for the nonlinear field amplitude dependence of the polarization hysteresis loops. In addition to this field dependence we assume a linear viscous interaction (again because the hysteresis loops have elliptic shapes for all frequencies and amplitudes) to be present in the polarization rotation model. Further on it is shown that this term will give rise to the hysteresis. Thus, we arrive at

$$
\begin{aligned}
\theta(E) & =\theta_{0, f}+\Delta \theta(E)+\gamma_{P} \Delta \dot{\theta}(E) \\
& =\theta_{0, f}+\beta_{1} E+\beta_{2} E^{2}+\beta_{3} E^{3}+\gamma_{P} \dot{E}\left(\beta_{1}+2 \beta_{2} E+3 \beta_{3} E^{2}\right) .
\end{aligned}
$$

The magnitude, sign and required number of the $\beta$-coefficients are dependent on the strain state and consequently the substrate, the deposition temperature, the composition of the film and the field range over which the angle fitting is performed $^{2}$. As the angle should decrease with increasing field, we expect a priori that the non-viscous parameters $\beta_{1}, \beta_{3}<0$, and the viscous parameter $\gamma_{P}<0$ because the response should lag behind the electric field, similar as for the strain response. The second order term $\beta_{2}$ gives rise to a possible asymmetry in the field sensitivity of $\Delta \theta(E)$. Since the magnitude of the viscous part is expected to be much smaller compared to non-viscous part, the angle-field dependence in Fig. $5.1 \mathrm{~d}$ ) has been fitted with Eq. 5.4 excluding the viscous terms. The fit values are given in Fig. $5.1 \mathrm{~d}$ ). It is seen that the expectations regarding the signs of the model parameters match the signs of the fitted values. In addition, the fit shows that $\beta_{2}>0$ and that

\footnotetext{
${ }^{2}$ In principle $\theta(E)$ can be calculated from a Landau-Devonshire description for a clamped film. In ref. $[48,49]$ this was done from a numerical evaluation of a Landau-Devonshire-model for $E=0$ and $200 \mathrm{kVcm}^{-1}$. However, since it is difficult to take into account the deformation of the unit cells in a polydomain film, we will here use the above phenomenological description and use the experimental fit parameters to describe $\theta(E)$.
} 
$\left|\beta_{1}\right|>\left|\beta_{2}\right|>\left|\beta_{3}\right|$. The fitted $\theta_{0, f}$ value indicates that the zero field polarization vector is rotated by $15.9^{\circ}$ from the rhombohedral body diagonal towards the [001]axis. X-ray diffraction analysis of the clamped PZT film, presented in the results section, shows that there is in-plane compressive strain of the unit cell, causing an out-of-plane tetragonal deformation of the cubic parent unit cell, leading to the observed rotation.

The dependence of the polarization to the angular change $\theta(E)$ can be rewritten as:

$$
\begin{aligned}
P(\theta(E)) & =P_{S} \cos \left(\theta_{0, f}+\Delta \theta(E)+\gamma_{P} \Delta \dot{\theta}(E)\right) \\
& =P_{r, f}\left[\cos \left(\Delta \theta(E)+\gamma_{P} \Delta \dot{\theta}(E)\right)-\tan \left(\theta_{0, f}\right) \sin \left(\Delta \theta(E)+\gamma_{P} \Delta \dot{\theta}(E)\right)\right]
\end{aligned}
$$

where we have defined the remanent polarization of the clamped film as $P_{r, f}=P_{S} \cos \left(\theta_{0, f}\right)$. As can be seen from Fig. $\left.5.1 \mathrm{~d}\right) \Delta \theta(E)$ is of the order of a few degrees only, therefore we can use lowest order Taylor expansions of the sin and cos terms, resulting in a nonlinear, but analytically tractable polynomial expression for the time-dependent polarization response:

$$
\begin{aligned}
P(t)=P_{r, f}\left[c_{0}\right. & +c_{1, s} E_{0} \sin (\omega t)+c_{1, c} E_{0} \cos (\omega t) \\
& +c_{2, s} E_{0}^{2} \sin (2 \omega t)+c_{2, c} E_{0}^{2} \cos (2 \omega t) \\
& +c_{3, s} E_{0}^{3} \sin (3 \omega t)+c_{3, c} E_{0}^{3} \cos (3 \omega t) \\
& +c_{4, s} E_{0}^{4} \sin (4 \omega t)+c_{4, c} E_{0}^{4} \cos (4 \omega t) \\
& +c_{5, s} E_{0}^{5} \sin (5 \omega t)+c_{5, c} E_{0}^{5} \cos (5 \omega t) \\
& \left.+c_{6, s} E_{0}^{6} \sin (6 \omega t)+c_{6, c} E_{0}^{6} \cos (6 \omega t)\right]
\end{aligned}
$$

The $c$-coefficients are functions of the $\beta$-coefficients and $E_{0}$ which are given in the Supporting Information Section A.3. Note that all coefficients are even functions of $E_{0}$. In addition, we give the values of the $c$-coefficients in the same section for $E_{0}=10 \mathrm{kV} \mathrm{cm}^{-1}$ and $f=3 \mathrm{kHz}$, and show that the amplitude of the harmonics rapidly decreases with increasing harmonic number.

The Fourier expansion shows that from the assumed $\theta(E)$ dependence one expects to measure in principle all even and odd order harmonics up to the $6^{\text {th }}$ harmonic, with hysteresis being present in all harmonics. Higher order polynomial fitting and/or expansion gives rise to higher order hysteresis and even higher order harmonics, although with rapidly decreasing amplitudes.

The first and higher order hysteresis loops are centered around the point $P_{\text {center }}=P_{r, f} c_{0}\left(E_{0} \omega\right) \approx P_{r, f}$. Within this model the amplitude of the $n^{t h}$ harmonic is given by

$$
\left|P_{n}\right|=P_{r, f}\left(E_{0}^{n} \sqrt{c_{n, s}^{2}+c_{n, c}^{2}}\right) .
$$


The amplitude of the hysteresis loop is dominated by the amplitude of the first harmonic. The average slope of the loop is the effective dielectric constant (or the Rayleigh parameter $m\left(E_{0}\right)$ of Eq. A.2 Supporting Information, given in the polarization rotation model by $\varepsilon_{\text {eff }} \approx P_{r, f} c_{1, s}$. We will see that experimentally the harmonics are not sharp delta functions in the frequency domain as assumed in the model but show considerable frequency spreading. Consequently, the energy contained in the different harmonics, which is proportional to $P_{n}^{2}$, is redistributed and the measured harmonic amplitudes $P_{n}$ at the harmonic frequencies $\omega_{n}=n \omega$ differ from the calculated values described by Eq. A.19 and Eq. A.20 Supporting Information. However, the type of dependence on field amplitude and frequency is expected to be conserved.

As the magnitude of the harmonics is the easiest parameter that can be extracted from the measurement, we give the expected scaling of $\left|P_{n}\right|$ with the amplitude of the electric field, following Eq. 5.7:

$$
\begin{aligned}
& \left|P_{1}\right|=P_{r, f}\left(E_{0} \sqrt{c_{1, s}^{2}+c_{1, c}^{2}}\right) \approx P_{r, f}\left(k_{1,1} E_{0}+k_{1,3} E_{0}^{3}\right) \\
& \left|P_{2}\right|=P_{r, f}\left(E_{0}^{2} \sqrt{c_{2, s}^{2}+c_{2, c}^{2}}\right) \approx P_{r, f}\left(k_{2,2} E_{0}^{2}+k_{2,4} E_{0}^{4}\right) \\
& \left|P_{3}\right|=P_{r, f}\left(E_{0}^{3} \sqrt{c_{3, s}^{2}+c_{3, c}^{2}}\right) \approx P_{r, f}\left(k_{3,3} E_{0}^{3}+k_{3,5} E_{0}^{5}\right) \\
& \left|P_{4}\right|=P_{r, f}\left(E_{0}^{4} \sqrt{c_{4, s}^{2}+c_{4, c}^{2}}\right) \approx P_{r, f}\left(k_{4,4} E_{0}^{4}+k_{4,6} E_{0}^{6}\right) \\
& \left|P_{5}\right|=P_{r, f}\left(E_{0}^{5} \sqrt{c_{5, s}^{2}+c_{5, c}^{2}}\right) \approx P_{r, f}\left(k_{5,5} E_{0}^{5}\right) \\
& \left|P_{6}\right|=P_{r, f}\left(E_{0}^{6} \sqrt{c_{6, s}^{2}+c_{6, c}^{2}}\right) \approx P_{r, f}\left(k_{6,6} E_{0}^{6}\right) .
\end{aligned}
$$

The $k_{n, m}$ coefficients are functions of the $\beta$-coefficients and $\omega$, where $n$ is the order of the harmonic and $m$ the power of the field amplitude. The exact expressions for the $k$-coefficients are given in the Supporting Information Section A.3, however these are upper limits of the harmonic amplitude, since much energy is leaked away lowering the measured polarization amplitudes and causing the frequency spreading of the harmonics. Eq. 5.8 predicts that the amplitude of the harmonics scale with an even or odd power polynomial of the field amplitude.

The loss tangent for the polarization can be written straightforwardly as the ratio of viscous to non-viscous part of the fundamental harmonic when the polarization signal is approximated by the fundamental harmonic as:

$$
P \approx P_{r, f}\left[c_{1, s} E_{0} \sin (\omega t)+c_{1, c} E_{0} \cos (\omega t)\right]
$$

The polarization loss tangent is then: 


$$
\tan \delta_{P} \approx\left|\frac{c_{1, c}}{c_{1, s}}\right| \approx\left|\frac{-\beta_{1} \gamma_{P} \omega\left[\left(\frac{3 \beta_{3} \tan \left(\theta_{0, f}\right)}{4 \beta_{1}}+\frac{3 \beta_{2}}{4}\right) E_{0}^{2}+\tan \left(\theta_{0, f}\right)\right]}{-\beta_{1}\left[\left(\frac{3 \beta_{2}}{4}+\frac{3 \beta_{3} \tan \left(\theta_{0, f}\right)}{4 \beta_{1}}\right) E_{0}^{2}+\tan \left(\theta_{0, f}\right)\right]}\right|=\left|\gamma_{P} \omega\right|
$$

This is the same expression as for the loss in the strain hysteresis. The loss tangent should be amplitude independent and should be a linear function of the frequency. This observation will become important in the discussion of the measured amplitude dependence of the polarization loss tangent in the results section.

\subsection{Experimental details}

\section{Film deposition}

The PZT film near the MPB with monoclinic crystal symmetry was grown by pulsed laser deposition (PLD) with a KrF excimer laser source (Lambda Physic, $248 \mathrm{~nm}$ wavelength). The target and film composition were determined by X-ray fluorescence (XRF) and Rutherford Back Scattering (RBS) to be $\mathrm{PbZr}_{0.55} \mathrm{Ti}_{0.45} \mathrm{O}_{3}$ (Kurt J. Lesker), hence in the rhombohedral range of the PZT phase diagram. The PZT film was sandwiched between two $100 \mathrm{~nm}$ thick $\mathrm{LaNiO}_{3}$ (LNO) electrodes, also deposited by PLD, on a $\mathrm{SrTiO}_{3}$ (STO) substrate. The PZT thickness was about $3 \mu \mathrm{m}$, calculated from the deposition rate and deposition time. The LNO electrodes were deposited with a laser repetition rate of $4 \mathrm{~Hz}$, energy density of $2.25 \mathrm{~J} \mathrm{~cm}^{-2}$, laser spot size of $3 \mathrm{~mm}^{2}$, target-to-substrate distance of $60 \mathrm{~mm}$, oxygen pressure of 0.1 mbar $\mathrm{O}_{2}$ and a substrate temperature of $600^{\circ} \mathrm{C}$. The PZT film was deposited with a laser repetition rate of $10 \mathrm{~Hz}$, energy density of $2 \mathrm{~J} \mathrm{~cm}^{-2}$, laser spot size of $3 \mathrm{~mm}^{2}$, target-to-substrate distance of $55 \mathrm{~mm}, 0.1 \mathrm{mbar} \mathrm{O}_{2}$ and at $600{ }^{\circ} \mathrm{C}$. The layers were deposited successively without breaking the vacuum. After deposition, the film was cooled down to room temperature in 1 bar $\mathrm{O}_{2}$ atmosphere at a cooling rate of $8^{\circ} \mathrm{C} \mathrm{min}^{-1}$. For ferro/piezoelectric measurements a $100 \mathrm{~nm}$ thick platinum (Pt) layer was deposited on top by sputtering, from which $300 \times 300 \mathrm{\mu m}^{2}$ top electrodes of the capacitors were patterned with a standard photolithography process and structured by argon ion beam etching of the top Pt and LNO layers. The bottom electrode is contacted by use of silver glue at the side of the sample.

\section{Analysis and Characterization}

Crystallographic properties of the PZT film were analyzed by a X-ray diffractometer (Bruker D8 Discover) equipped with a high brilliance microfocus $\mathrm{Cu}$ rotating anode generator, Montel optics, a Ge (220) two-bounce monochromator, a double pinhole beam collimator with a diameter of $200 \mu \mathrm{m}$, and an area detector (EIGER2 $\mathrm{R} 500 \mathrm{~K})$. The asymmetric reciprocal space maps were reconstructed from sets of high-resolution rocking curves. All XRD measurements shown in this work were performed on a single, unpoled capacitor. 
The piezoelectric and ferroelectric properties were measured with a double beam laser interferometer (aixDBLI), to eliminate the influence of substrate bending, utilizing a ferroelectric tester (aixACCT TF-2000 Analyzer) to measure the ferroelectric properties. The large field polarization and strain (global P-E and S-E, respectively) loop measurements were performed using a sinusoidal AC-electric field with an amplitude of $200 \mathrm{kV} \mathrm{cm}^{-1}$ at $3 \mathrm{kHz}$. The coercive field of the film was around $24 \mathrm{kV} \mathrm{cm}^{-1}$. The small signal $d_{33, f, s s}$ measurements were measured using a lock-in technique with a DC-driving field in the range of $200 \mathrm{kV} \mathrm{cm}^{-1}$ and an AC peak-topeak amplitude of $1.6 \mathrm{kV} \mathrm{cm}^{-1}$ at a frequency of $3 \mathrm{kHz}$.

The sub-coercive field hysteresis behavior was measured using 20 cycles of sinusoidal signal with peak-to-peak amplitude ranging from $2.5 \mathrm{kV} \mathrm{cm}^{-1}$ to $10 \mathrm{kV} \mathrm{cm}^{-1}$, i.e. up to about half of the coercive field. The measurement frequency was varied from $70 \mathrm{~Hz}$ to $5 \mathrm{kHz}$. Before each sub-coercive field measurement the film was poled with a DC field of $200 \mathrm{kV} \mathrm{cm}^{-1}$, to realize comparable starting conditions. The sub-coercive field polarization and strain responses were measured simultaneously, at room temperature and with zero bias fields.

\subsection{Results and discussion}

The polarization rotation model considers a monoclinic unit cell and predicts the observed scaling of strain and polarization with driving field amplitude and frequency. First, we present and discuss the measurements on the crystal structure, showing that the investigated film is composed of compressively strained, monoclinic, polydomain PZT. Secondly, the data treatment procedure, which is required to obtain strain and polarization information from generally noisy and drifting raw measurement data, is presented. The film used in the experiments was a $3 \mu \mathrm{m}$ thick PZT film, with 55/45 Zr/Ti ratio, sandwiched between LNO electrodes. All layers were grown subsequently by pulsed laser deposition on a (001)-oriented STO substrate without breaking the vacuum. The strain and polarization were measured with a double beam laser interferometer (aixDBLI) combined with the aixACCT TF-2000 Analyzer. See the Experimental section for more information about the experimental procedures. Finally, we present the analysis of the treated strain and polarization hysteresis measurement data in terms of the polarization rotation model.

\subsubsection{Crystal structure}

X-ray diffraction (XRD) analysis was performed on the LNO/PZT/LNO heterostructure as explained in the Supporting Information Section A.4. The data could be best fitted with a pseudo-rhombohedral unit cell elongated in the (001)-direction (from crystallographic point of view monoclinic), with a four-fold in-plane symmetry, i.e. the unit cells are rotated by integer multiples of $90^{\circ}$ with respect to each other. The PZT in-plane unit-cell parameters are $a_{u c}=b_{u c}=4.081 \AA$, and the out-of-plane lattice parameter is $c_{u c}=4.097 \AA$. Here the subscript $u c$ is used with unit-cell parameters $a, b$ and $c$ to distinguish them from other parameters with the same 
symbols. The angle between the in-plane lattice vectors is obtained as $\gamma_{u c} \approx 89.79^{\circ}$, while the angles between in-plane and out-of-plane are $\alpha_{u c}=\beta_{u c} \approx 89.74^{\circ}$. The c-axis of all unit cells is perpendicular to the substrate, hence the (001)-planes are tilted by $0.26^{\circ}$. The unit cell volume is $V_{u c} \approx a_{u c}^{2} c_{u c}=68.27 \AA^{3}$ and the pseudocubic lattice parameter is given by $a_{p c}=V_{p c}^{1 / 3}=4.087 \AA$. Because of the monoclinic lattice symmetry, the polarization vector is expected to rotate in the (110)-plane of each unit cell [51]. The structure appears to have been pushed to the monoclinic side, which might be due to a high density of point defects, indicated by the slightly higher unit cell volume, and the clamping to the substrate, introducing strain. The small tetragonality ratio $c_{u c} / a_{u c}=1.004$ is a consequence of the in-plane compressive strain, due to the difference in thermal expansion of the film and substrate, and is estimated as $\varepsilon_{\mathrm{ip}}=\left(a_{u c}-a_{p c}\right) / a_{p c}=-0.13 \%$ [52]. For films thicker than a few hundred $\mathrm{nm}$, the substrate-induced strain is mainly due to the difference in thermal expansion coefficients between film and substrate, since at deposition temperature the bulk of the film can be assumed to be fully relaxed from epitaxial strain due to the incorporation of (dislocation) defects. The room temperature strain, caused by the difference in thermal expansion coefficients $\left(4.6 \mathrm{ppm} \mathrm{K}^{-1}\right.$ for the PZT 55/45 film, which is obtained by interpolation of the data for PZT 60/40 and PZT 50/50 films, and $11.7 \mathrm{ppm} \mathrm{K}^{-1}$ for the STO substrate) when cooling down from deposition to room temperature and corrected for the volume increase due to the paraelectricto-ferroelectric phase transition, is calculated as $S_{m}^{0} \approx-0.003[48,49]$. This explains the compressive strain in the film deduced from the XRD measurements.

\subsubsection{Measured strain and polarization response}

Fig. 5.2 shows in the left and right column subfigures the raw data in time and frequency domains, of respectively the applied electric field $E=E_{0} \sin (\omega t)$, the measured strain $S$ and the measured polarization $P$ response, for a measurement frequency of $f=\omega / 2 \pi=3 \mathrm{kHz}$ and electric field amplitude of $E_{0}=10 \mathrm{kV} \mathrm{cm}^{-1}$. Fig. $5.2 \mathrm{a}$ ) shows 20 periods of the applied signal ${ }^{3}$. The Fourier spectrum of the applied signal, Fig. $5.2 \mathrm{~b}$ ), shows that the applied signal only consists of the $3 \mathrm{kHz}$ fundamental harmonic and has no higher order frequency components. This check ensures that any observed higher harmonic signal does not originate from the driving signal. The input signal-to-noise ratio (SNR) visible in Fig. $5.2 \mathrm{~b}$ ) is about $10^{3}$.

The raw strain data in Fig. 5.2c) is noisy and subject to drift in the time domain. In the frequency domain, Fig. $5.2 \mathrm{~d}$ ), the fundamental frequency of $3 \mathrm{kHz}$ is clearly observed and no higher harmonics can be identified apart from some random noise peaks, which we attribute to numerical artefacts in the Fast Fourier Transform algorithm, since they have a very small peak width of only one frequency step. Below $2 \mathrm{kHz}$ there is frequency dependent noise, which explains the drift in the time domain. The measured polarization signal, Fig. 5.2 e), has a constant am-

\footnotetext{
${ }^{3}$ The applied signal does not always start at 0 for $t=0$ as assumed in the model. To compare the results with the model, we fit the applied signal with a phase shift sine wave for each measurement to extract the initial phase shift $\phi$, that is in the range $\pm 3^{\circ}$ for the used amplitudes and frequencies. This additional step is needed to exclude any non-material induced phase change.
} 
plitude of $1.35 \mathrm{\mu C} \mathrm{cm}^{-2}$, but also drifts in time. Note that the amplitude of the polarization signal is small compared to the value of the remanent polarization, $P_{r}=38.6 \mu \mathrm{C} \mathrm{cm}^{-2}$, as we only measure the change of the polarization. In the frequency domain, Fig. 5.2f), one observes next to the fundamental frequency clearly higher harmonics, up to $5^{\text {th }}$ order, superimposed on a decreasing frequency dependent noise baseline. The baseline was fitted with a linear function in the log-log plot shown in Fig. 5.2f), using the power-law dependence $P(f)=a f^{b}$ with $a$ and $b$ being some fit parameters. For the complete data set, i.e. for all amplitudes and frequencies, the exponent was obtained as $b=0.92 \pm 0.03$, demonstrating the $1 / f$ character of the noise in the polarization measurements. The $1 / f$-noise baseline extracted from the Fourier spectrum of the polarization signal, has been overlaid to the strain data in Fig. $5.2 \mathrm{~d}$ ), showing a good match with the frequency dependent baseline in the strain. This observation suggests that the observed $1 / f$-noise in both measurements has the same origin. The $1 / f$ dependence probably can tell something about the interaction mechanism of polarization and strain, but this is beyond the scope of this paper. The observed white noise in the strain at higher frequencies is not observed in the polarization and is thought to be linked to higher noise level in the displacement measurement method.

\subsubsection{Strain data}

The drift, i.e. low frequency noise in the strain data hinders an accurate fit of the model to the raw measurement data since the model predicts a purely sinusoidal strain signal. Therefore, the strain signal is filtered in the frequency domain by applying a bandpass filter around the fundamental harmonic. The filtered data is transformed back into the time domain and then averaged over the 20 measured cycles to increase the SNR. The averaged strain signal is plotted with markers with respect to time in Fig. $5.3 \mathrm{a}$ ) and electric field in Fig. $5.3 \mathrm{~b}$ ). The number of datapoints in one cycle was 128 but only every fifth datapoint is shown for clarity. The standard deviation of the averaged data was too small to be shown in the figure, as the associated error bars would be smaller than the datapoint markers. The hysteresis loops in Fig. 5.3 b) are elliptical, in contrast to the lenticular loops, arising from the Rayleigh theory. The filtered and averaged strain data at $3 \mathrm{kHz}$ in the time domain has been fitted with Eq. 5.2 using the inverse of the standard deviations as the weighting factors and shown by the continuous lines in Fig. 5.3 a) and Fig. $5.3 \mathrm{~b}$ ). The data and fits for all measured frequencies for 3 selected amplitudes are shown in Supporting Information Section A.7 . The model fits the data points perfectly and all loops are traversed counterclockwise, indicating that $\gamma_{d}$ is negative, as is expected.

The extracted model parameters $d_{33, f}$ and $\gamma_{d}$ are plotted versus $E_{0}$ in Fig. 5.4 a) and Fig. $5.4 \mathrm{~b}$ ). The statistical uncertainty of individual model parameters is smaller than the datapoint markers and are therefore not shown. Both model parameters appear to fluctuate around an average value, independent of the field amplitude. To verify this observation, the average value and standard deviation $\sigma$ (half of the red confidence intervals of the model parameters shown in Fig. 5.4 a) and Fig. 5.4 b) 

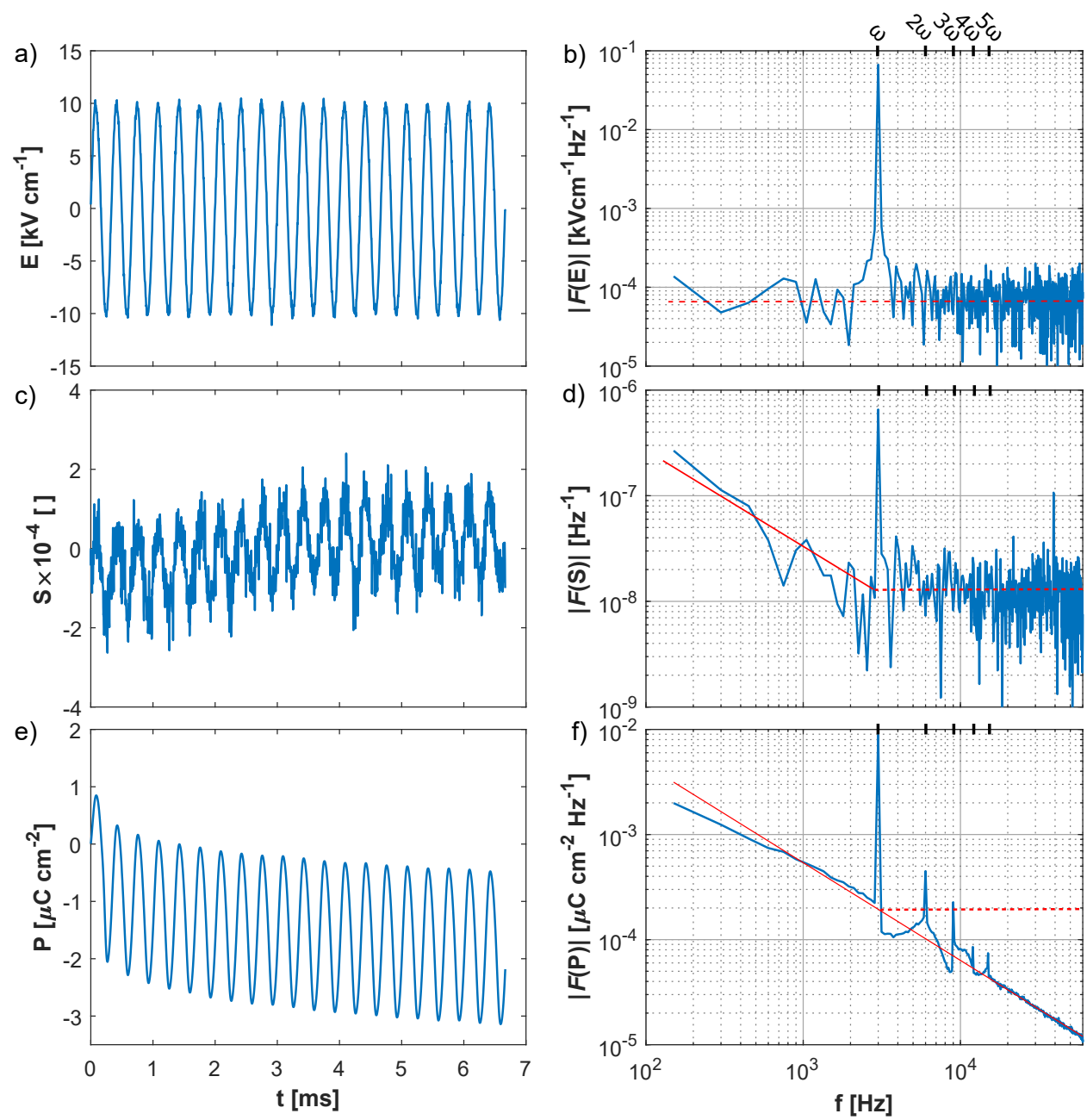

Figure 5.2: a, b) Applied field $E$, c, d) measured strain $S$ and e, f) polarization $P$ for an amplitude of $10 \mathrm{kV} \mathrm{cm}^{-1}$ and a frequency of $3 \mathrm{kHz}$ in time (left) and frequency domains (right). Solid red line is the fitted $1 / f$ noise and dashed red line is the noise baseline.

are propagated to the strain, resulting in a spread of $6 \%$. This spread is below the $10 \%$ measurement-to-measurement accuracy of our strain measurements. This supports the conjecture that our model parameters are amplitude independent within the measurement accuracy of the used DBLI system. The average values of the fit parameters over the measured field range are $d_{33, f}(3 \mathrm{kHz})=96 \mathrm{pm} \mathrm{V}^{-1}$ and $\gamma_{d}(3 \mathrm{kHz})=-9 \times 10^{-11} \mathrm{~cm} \mathrm{kV}^{-1} \mathrm{~Hz}^{-1} \mathrm{rad}^{-1}$. An independent measurement of the small signal piezoelectric constant at the same frequency measures $d_{33, f, s s}(3 \mathrm{kHz})=93 \mathrm{pm} \mathrm{V}^{-1}$ as shown in Fig. A.5 Supporting Information, is in good agreement with the value extracted from the hysteresis measurements. Both values are in agreement with $d_{33, f}$ values of PZT films with similar stoichiometry, 

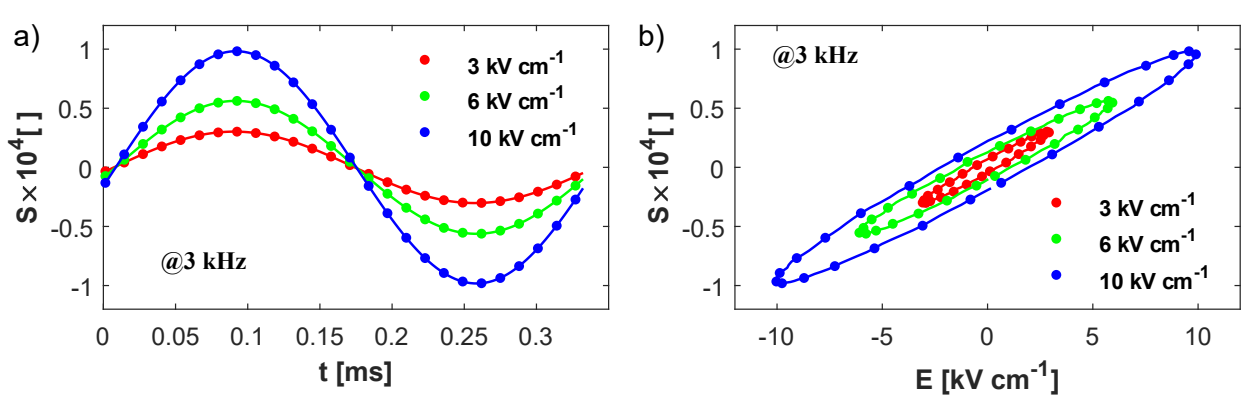

Figure 5.3: a) Filtered and averaged strain signal plotted with respect to time, and b) electric field, for three different field amplitudes at $3 \mathrm{kHz}$. Datapoints show the measurements and the lines are obtained from fitting with Eq. 5.2.

reported in literature [28, 53]. In Fig. 5.4c) the loss tangent values for different excitation amplitudes, calculated from the hysteresis loop area, are shown with markers. In the same figure, the line denotes the average loss tangent calculated from Eq. 5.3 using average values of $d_{33, f}(3 \mathrm{kHz})$ and $\gamma_{d}(3 \mathrm{kHz})$. The confidence interval is obtained from the spread of values in these parameters. The loss tangent calculated from the area falls well within the confidence interval predicted by the model. Within measurement accuracy we can conclude that the strain loss tangent is independent of the amplitude, as was predicted by the model if $d_{33, f}$ and $\gamma_{d}$ are independent of the excitation amplitude.

Fig. $5.5 \mathrm{a}$ ) and Fig. $5.5 \mathrm{~b}$ ) show the average values of $d_{33, f}$ and $\gamma_{d}$ over the measured field range for different measurement frequencies and the error bars denote the standard deviation. Within the measurement range, the $d_{33, f}(f)$ data is practically frequency independent, so the weighted average and the corresponding confidence interval can be calculated. The field and frequency averaged value is $d_{33, f, a v}=98 \mathrm{pm} \mathrm{V}^{-1}$ that is denoted by the red line in Fig. $5.5 \mathrm{a}$ ). $\gamma_{d}$ in Fig. $5.5 \mathrm{~b}$ ) does not show a frequency dependence if one neglects the low frequency data. The latter have large error bars originating from the dominant $1 / f$-noise at these frequencies.

The weighted average of all data points is $\gamma_{d, a v}=-10 \times 10^{-11} \mathrm{~cm} \mathrm{kV}^{-1} \mathrm{~Hz}^{-1} \mathrm{rad}^{-1}$. This value is very close to the high frequency data therefore we consider $\gamma_{d}$ to be frequency independent over the whole measured frequency range. As both extracted parameters are amplitude and frequency independent, the viscosity constant $\gamma_{S}$ is given by $\gamma_{s, a v} \approx-10 \times 10^{-6} \mathrm{~Hz}^{-1} \mathrm{rad}^{-1}$. Shown in Fig. $5.5 \mathrm{c}$ ) is the loss tangent as function of frequency calculated from the area of the hysteresis loops using Eq. A.7 Supporting Information. The error bars again denote the standard deviation in the loop area for the measurements at different frequencies. The red line is calculated from Eq. 5.3 with the average values $d_{33, f, a v}$ and $\gamma_{d, a v}$ and the error corridor is the corresponding $\pm 2 \sigma$ confidence interval, due to error propagation of the confidence intervals of Fig. $5.5 \mathrm{a}$ ) and Fig. $5.5 \mathrm{~b}$ ). The good linear scaling with frequency shows that the loss tangent is well described by Eq. 5.3, supporting the validity of the viscous polarization rotation model. Further it indicates again that the param- 

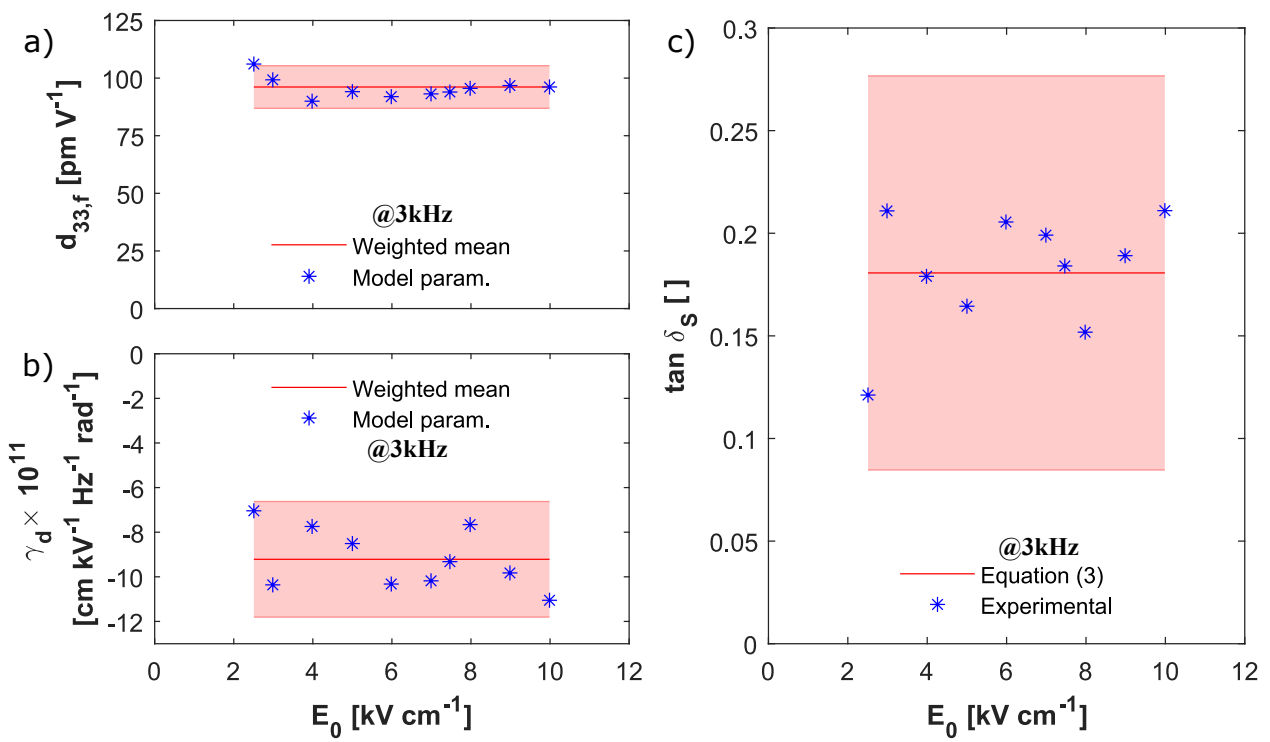

Figure 5.4: Field amplitude dependence of the extracted model parameters a) $d_{33, f}$, and b) $\gamma_{d}$. The red line denotes the weighted average of the model parameters, the red error corridor indicates the $\pm 2 \sigma$ confidence intervals. c) $\tan \delta_{S}$ calculated from the area of the S-E hysteresis loops. The red line and confidence interval are calculated with model Eq. 5.3 using data from a) and b).

eters $d_{33, f}, \gamma_{d}$ and resulting $\gamma_{S}$ are independent of field amplitude and frequency. The Rayleigh model with viscous loss (see Supporting Information Section A.1) can explain the observed frequency dependency, but would predict a strong amplitude dependency of the loss tangent as in Eq. A.9 Supporting Information, which is absent in our measurement data.

To the best of our knowledge, in literature there is no experimental data for films of similar composition and the same amplitude and frequency, that would allow a direct comparison of the loss tangent with our results. However, the results of Robert et al. allow a qualitative comparison [54]. They found a viscous loss tangent contribution of $0.044 \pm 0.004$, using the viscous Rayleigh model for the direct piezoelectric effect in a PZT ceramic (with composition near the morphotropic phase boundary) measured at $35 \mathrm{~Hz}$. This value is comparable in magnitude to our value at $70 \mathrm{~Hz}$ of $0.020 \pm 0.013$. However, Robert et al. found this viscous term in addition to a dominant amplitude dependent Rayleigh term that is increasing up to 0.2 for the largest field amplitude. In our case such an amplitude dependent Rayleigh loss mechanism is not found. This difference is of interest since it indicates that the differences in loss behavior between our film and the ceramics can be attributed to differences in crystal quality. Especially the number of defects in our epitaxial films is expected to be considerably smaller than in polycrystalline ceramics. Furthermore, the character of defects changes from dominantly lattice defects and vacancies for epitaxial films to grain boundaries in polycrystalline materials. This change in the character and the number of defects is expected to change the 

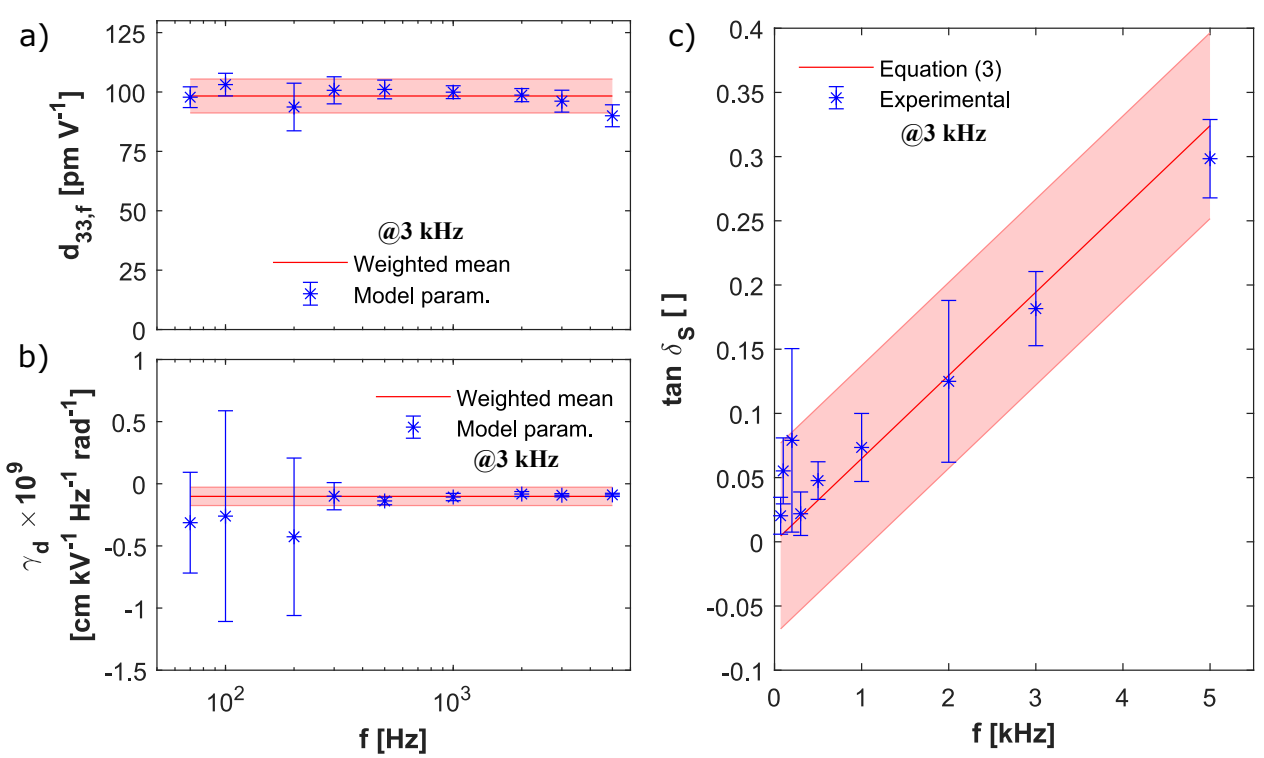

Figure 5.5: a) Averaged longitudinal piezoelectric coefficient $d_{33, f}$ and b) the viscosity parameter $\gamma_{d}$ for all field amplitudes $E_{0}$. c) Loss tangent calculated from the area of the hysteresis loops. The red line is calculated with the field and frequency averaged values of $d_{33, f, a v}$ and $\gamma_{S, a v}$, using Eq. 5.3. The error bars indicate the $\pm \sigma$ standard deviation of the data in all figures.

amplitude dependence of the loss. In polycrystalline materials the loss is dominated by the amplitude dependent part stemming from Barkhausen jumps of the pinned domain walls in addition to the amplitude independent viscous part observed in both systems [54].

\subsubsection{Polarization data}

To extract the amplitude of the harmonics out of the polarization data, the peak value of the corresponding peak in the Fourier spectrum is taken. In Fig. 5.6 the thus determined amplitudes $\left|P_{i}\right|$ of the $1^{\text {st }}$ to $5^{\text {th }}$ harmonic are shown as function of the field amplitude. The vertical scale for the $2^{\text {nd }}$ to $5^{\text {th }}$ harmonic is magnified for clarity. The solid lines correspond to the fits using the predicted dependencies on $E_{0}$ for the various harmonics according to Eq. 5.8. The model is in very good agreement with the field dependence of the experimental data of the lower harmonics. The poorer fits for the $4^{t h}$ and $5^{t h}$ harmonic at low amplitudes are due to the low SNR for these harmonics. For Rayleigh behavior, the first harmonic is expected to scale as $P_{1} \propto a_{1} E_{0}+a_{2} E_{0}^{2}$ (with $a_{1}$ and $a_{2}$ some field independent constants), whereas in the polarization rotation model the dependence is of third order, $P_{1} \approx P_{r, f}\left(k_{1,1} E_{0}+k_{1,3} E_{0}^{3}\right)$ [37]. Further, the Rayleigh model predicts that no even harmonics are present, which are clearly observed here. It predicts also that all other uneven harmonics scale with $E_{0}^{2}$, in contrast to $k_{n, n} E_{0}^{n}+k_{n, n+2} E_{0}^{n+2}$-scaling seen here. Although the first harmonic data might be fitted fairly well with the Rayleigh model, it is clear that the Rayleigh model completely fails to describe the 
field scaling of all other observed harmonics and the presence of the even harmonics.

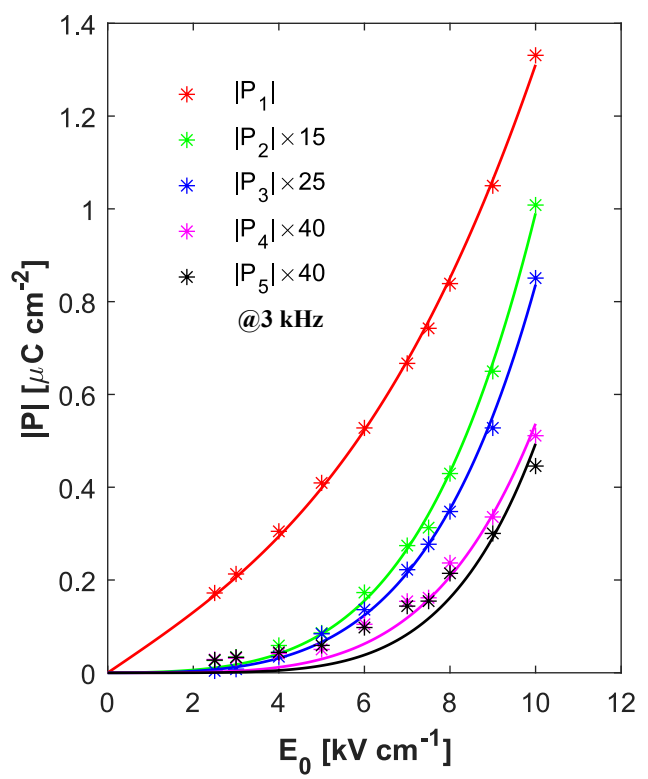

Figure 5.6: Scaling of the amplitude of the polarization harmonics with respect to the field amplitude $E_{0}$ at an excitation frequency of $3 \mathrm{kHz}$. The scaling of the $2^{\text {nd }}$ to $5^{\text {th }}$ harmonic is magnified for clarity. The data points are denoted by the stars and the lines are fits with Eq. 5.8).

As discussed, within the polarization rotation model the loss behavior can be approximated by the fundamental harmonic only. Fig. $5.7 \mathrm{a}$ ) and Fig. $5.7 \mathrm{~b}$ ) show the polarization hysteresis at selected amplitudes for $3 \mathrm{kHz}$. The data for all measured frequencies are shown in Supporting Information Section A.8. The data in these figures was obtained by bandpass filtering at all harmonic frequencies and then averaging over the measured 20 cycles. As for the strain the standard deviation calculated from the averaging is smaller than the marker size. Note that all higher harmonics are included in these loops. The experimental loops were fitted with the fundamental harmonic only, using Eq. 5.9 and taking into account the inherent phase shift present in the applied signal ${ }^{4}$. The good fits in time and field domain respectively show that, although the higher harmonics can be clearly observed in the frequency domain, their contributions are not visible in time or field domain except at the peaks of the $10 \mathrm{kV} \mathrm{cm}^{-1}$ amplitude measurement.

The loss tangent is proportional to the area of the P-E loop and inversely proportional to the polarization value at maximum field, therefore it can be concluded that the loss tangent calculated from the fundamental harmonic can accurately de-

\footnotetext{
${ }^{4}$ The applied signal does not always start at 0 for $t=0$ as assumed in the model. To compare the results with the model, we fit the applied signal with a phase shift sine wave for each measurement to extract the initial phase shift $\phi$, that is in the range $\pm 3^{\circ}$ for the used amplitudes and frequencies. This additional step is needed to exclude any non-material induced phase change.
} 

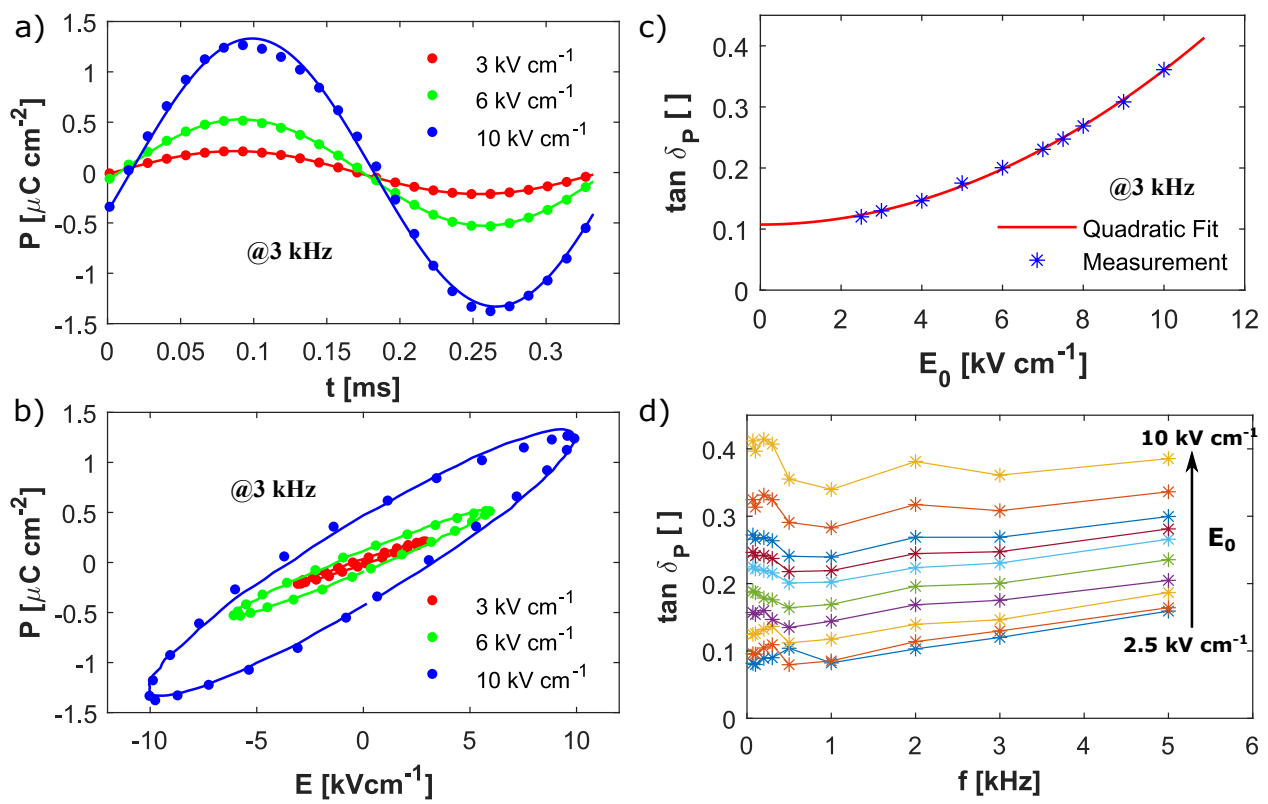

Figure 5.7: a) Filtered and averaged polarization signal plotted with respect to time, and b) electric field, for 3 different field amplitudes at $3 \mathrm{kHz}$. The markers represent the measured data, which includes all higher harmonics, and the solid lines are obtained by fitting with Eq. 5.9. c) Loss tangent, calculated using Eq. 5.10, is shown with markers. The red line denotes a fit with quadratic amplitude scaling as explained in detail in the main text. d) Frequency dependence of the loss tangent for all used amplitudes.

scribe the field scaling of the loss. Fig. $5.7 \mathrm{c}$ ) shows the amplitude dependence of the thus obtained loss tangent. The field dependence can be best fitted with a quadratic function with a constant offset of the form, $\tan \delta_{P}\left(E_{0}, \omega\right)=\tan \delta_{P}(0, \omega)\left(1+c E_{0}^{2}\right)$ with positive $c$, as shown by the red curve. The polarization rotation model in Eq. 5.10 predicts no explicit field dependence for the loss tangent. The field dependence can only be implicit to the viscosity parameter with the same quadratic form $\gamma_{P}\left(E_{0}, \omega\right)=\gamma_{P}(0, \omega)\left(1+c E_{0}^{2}\right)$. From the loss tangent curve in Fig. $\left.5.7 \mathrm{c}\right)$ at frequency $\omega_{0}=2 \pi \times 3 \mathrm{kHz}$ one finds the model parameters, loss tangent at zero field as $\tan \delta_{P}\left(0, \omega_{0}\right)=0.11, c=0.024 \mathrm{kV}^{-2} \mathrm{~cm}^{2}$ and thus

$\gamma_{P}\left(0, \omega_{0}\right)=-5.84 \times 10^{-6} \mathrm{~Hz}^{-1} \mathrm{rad}^{-1}$. Interestingly $\gamma_{P}\left(0, \omega_{0}\right)$ is nearly equal in value to $\gamma_{S}\left(0, \omega_{0}\right)$, which is in-line with the expectation of Landau-Devonshire model that $\gamma_{P}$ and $\gamma_{S}$ should be identical. However, in contrast to $\gamma_{S}$ being field independent, $\gamma_{P}$ shows a strong quadratic field dependence. We attribute the linearity of $\gamma_{S}$ to the 4 orders of magnitude smaller relative change of strain compared to polarization.

The frequency dependencies of the loss tangent for all used field amplitudes are shown in Fig. $5.7 \mathrm{~d}$ ). A linear increase is observed for frequencies above $500 \mathrm{~Hz}$, which is typical for a viscous loss process and is as described by the proposed polarization rotation model. The deviation for lower frequencies may be explained 
by the fact that for these frequencies the coercive field is lower, so that the field is swept up to $3 / 4 E_{c}$ or even higher as can be seen in Tab. A.2 Supporting Information. The nonlinearity is then larger, which changes the values of the $\beta$-parameters and thus of the loss. The observed amplitude and frequency dependency of the loss tangent for the polarization deviates clearly from the predicted dependencies of the Rayleigh model, as it is also the case for the strain.

\subsection{Conclusions}

In summary, we observe experimentally a hysteretic behavior with linear dependence of the strain on the field for the strain and a nonlinear hysteretic polarization behavior for an epitaxial PZT film with monoclinic symmetry. The nonlinearity is characterized by the presence of all even and odd order harmonics observable up to $5^{\text {th }}$ order in the polarization measurements. These findings are explained by a model that relates the rotation of the polarization vector in the (110)-plane of a pseudo cubic unit cell in response to an applied electric field, as is expected for the used PZT film with monoclinic symmetry. The polarization rotation is accompanied by viscous interactions for the strain and polarization response of the film. The strain behavior is described by a linear field dependent stretching of the unit-cell due to the rotation of the polarization vector. This strain model describes in detail the field amplitude and frequency dependence of the measured strain and loss tangent. The polarization is described by the rotation of the polarization vector, which has a nonlinear dependence on the field, and a viscous interaction. The model predicts all of the observed even and odd harmonics and their amplitude scaling. The polarization rotation model takes into account the crystal symmetry of the unit cell, as it is based on the rotation of the polarization vector which is characteristic for monoclinic crystal symmetry. Hence it is expected to be applicable to other material systems in which polarization rotation is present, such as in monoclinic or rhombohedral crystal symmetries. This expectation can be true for the rhombohedral crystal symmetry only if the unit cell transforms into monoclinic symmetry under an externally applied field.

\section{Acknowledgements}

This work is part of the research programme "Smart Multilayer Interactive Optics for Lithography at Extreme UV wavelengths (SMILE)", with contract number 10448 and financial support by the Dutch Research Council (NWO) and Carl Zeiss SMT. The authors acknowledge the support of the Industrial Focus Group XUV Optics and Inorganic Material Science Group, carried out at the MESA + Institute for Nanotechnology at the University of Twente and further support by the Province of Overijssel, ASML, and Malvern Panalytical. The RBS measurements were performed by Dr. Max Doebeli of the Ion Beam Physics group of the ETH Zurich, Switzerland. 


\section{References}

[1] R. Waser, Ceramic Materials for Electronics; Processing, Properties, and Applications, in Advanced Materials, Vol. 4 (Wiley, New York 1991, 1992) Book section 311, pp. 311-311, 2nd ed.

[2] N. Setter, D. Damjanovic, L. Eng, G. Fox, S. Gevorgian, S. Hong, A. Kingon, H. Kohlstedt, N. Y. Park, G. B. Stephenson, I. Stolitchnov, A. K. Taganstev, D. V. Taylor, T. Yamada, and S. Streiffer, Ferroelectric thin films: Review of materials, properties, and applications, Journal of Applied Physics 100, 051606 (2006).

[3] P. Muralt, Recent Progress in Materials Issues for Piezoelectric MEMS, Journal of the American Ceramic Society 91, 1385 (2008).

[4] P. Muralt, R. G. Polcawich, and S. Trolier-McKinstry, Piezoelectric Thin Films for Sensors, Actuators, and Energy Harvesting, MRS Bulletin 34, 658 (2009).

[5] C.-B. Eom and S. Trolier-McKinstry, Thin-film piezoelectric MEMS, MRS Bulletin 37, 1007 (2012).

[6] M. Bayraktar, A. Chopra, G. Rijnders, K. Boller, and F. Bijkerk, Wavefront correction in the extreme ultraviolet wavelength range using piezoelectric thin films, Optics Express 22, 30623 (2014).

[7] M. Nematollahi, P. Lucke, M. Bayraktar, A. Yakshin, G. Rijnders, and F. Bijkerk, Nanoscale piezoelectric surface modulation for adaptive extreme ultraviolet and soft x-ray optics, Optics Letters 44, 5104 (2019).

[8] M. Bayraktar, W. A. Wessels, C. J. Lee, F. A. van Goor, G. Koster, G. Rijnders, and F. Bijkerk, Active multilayer mirrors for reflectance tuning at extreme ultraviolet (EUV) wavelengths, Journal of Physics D: Applied Physics 45, 494001 (2012).

[9] B. Jaffe, W. R. Cook, and H. Jaffe, Chapter 7 - Solid Solutions of Pb(Ti, Zr, $\mathrm{Sn}, \mathrm{Hf}) \mathrm{O}_{3}$, in Piezoelectric Ceramics, edited by B. Jaffe, W. R. Cook, and H. Jaffe (Academic Press, 1971) pp. 135-183.

[10] P. Muralt, Ferroelectric thin films for micro-sensors and actuators: a review, Journal of Micromechanics and Microengineering 10, 136 (2000).

[11] S. Trolier-McKinstry and P. Muralt, Thin Film Piezoelectrics for MEMS, Journal of Electroceramics 12, 7 (2004).

[12] H. Yang, F. Yan, Y. Lin, and T. Wang, Novel Strontium Titanate-Based Lead-Free Ceramics for High-Energy Storage Applications, ACS Sustainable Chemistry \& Engineering 5, 10215 (2017).

[13] J. O. Gentner, P. Gerthsen, N. A. Schmidt, and R. E. Send, Dielectric losses in ferroelectric ceramics produced by domain-wall motion, Journal of Applied Physics 49, 4485 (1978). 
[14] B. Laikhtman, Flexural vibrations of domain walls and dielectric dispersion of ferroelectrics, Physics of the Solid State 15, 62 (1973).

[15] V. Postnikov, V. Pavlov, S. Gridnev, and S. Turkov, Interaction between $90^{\circ}$ domain walls and point defects of the crystal lattice in ferroelectric ceramics, Physics of the Solid State 10, 1267 (1968).

[16] V. Postnikov, V. Pavlov, S. Gridnev, B. Darinskii, and I. Glozman, Internal friction in $\mathrm{Pb}_{0.95} \mathrm{Sr}_{0.05}\left(\mathrm{Zr}_{0.53} \mathrm{Ti}_{0.47}\right) \mathrm{O}_{3}+3 \% \mathrm{PbO}$ ferroelectric ceramic, Bulletin of the Russian Academy of Sciences: Physics 31, 1888 (1967).

[17] G. Arlt and H. Dederichs, Complex elastic, dielectric and piezoelectric constants by domain wall damping in ferroelectric ceramics, Ferroelectrics 29, 47 (1980).

[18] L. Rayleigh, XXV. Notes on electricity and magnetism.-III. On the behaviour of iron and steel under the operation of feeble magnetic forces, The London, Edinburgh, and Dublin Philosophical Magazine and Journal of Science 23, 225 (1887).

[19] L. Néel, Théorie des lois d'aimantation de Lord Rayleigh, Cahiers de physique 12, 1 (1942).

[20] L. Néel, Théorie des lois d'aimantation de Lord Rayleigh, 2, Cahiers de physique 13, 18 (1943).

[21] D. Damjanovic and M. Demartin, The Rayleigh law in piezoelectric ceramics, Journal of Physics D: Applied Physics 29, 2057 (1996).

[22] G. Bertotti, V. Basso, and G. Durin, Random free energy model for the description of hysteresis, Journal of Applied Physics 79, 5764 (1996).

[23] G. Bertotti, Energetic and thermodynamic aspects of hysteresis, Physical Review Letters 76, 1739 (1996).

[24] D. A. Hall, Review Nonlinearity in piezoelectric ceramics, Journal of Materials Science 36, 4575 (2001).

[25] D. V. Taylor and D. Damjanovic, Evidence of domain wall contribution to the dielectric permittivity in PZT thin films at sub-switching fields, Journal of Applied Physics 82, 1973 (1997).

[26] D. Damjanovic, Logarithmic frequency dependence of the piezoelectric effect due to pinning of ferroelectric-ferroelastic domain walls, Physical Review B: Condensed Matter and Materials Physics 55, R649 (1997).

[27] D. Damjanovic, S. S. N. Bharadwaja, and N. Setter, Toward a unified description of nonlinearity and frequency dispersion of piezoelectric and dielectric responses in $\mathrm{Pb}(\mathrm{Zr}, \mathrm{Ti}) \mathrm{O}_{3}$, Materials Science \& Engineering, B: Advanced Functional Solid-State Materials 120, 170 (2005).

[28] K. Vergeer, Structure and functional properties of epitaxial $\mathrm{PbZr}_{x} \mathrm{Ti}_{1-x} \mathrm{O}_{3}$ films, Phd thesis (2017). 
[29] F. Preisach, Über die magnetische Nachwirkung die magnetische Nachwirkung, Zeitschrift für Physik 94, 277 (1935).

[30] G. Robert, D. Damjanovic, and N. Setter, Preisach distribution function approach to piezoelectric nonlinearity and hysteresis, Journal of Applied physics 90, 2459 (2001).

[31] G. Robert, D. Damjanovic, N. Setter, and A. Turik, Preisach modeling of piezoelectric nonlinearity in ferroelectric ceramics, Journal of Applied Physics 89, 5067 (2001).

[32] D. Damjanovic, Hysteresis in piezoelectric and ferroelectric materials, in The Science of Hysteresis, edited by G. Bertotti and I. D. Mayergoyz (Academic Press, Oxford, 2006) Book section 4, pp. 337-465.

[33] D. V. Taylor and D. Damjanovic, Piezoelectric properties of rhombohedral $\mathrm{Pb}(\mathrm{Zr}, \mathrm{Ti}) \mathrm{O}_{3}$ thin films with (100), (111), and "random" crystallographic orientation, Applied Physics Letters 76, 1615 (2000).

[34] A. Pramanick, D. Damjanovic, J. C. Nino, and J. L. Jones, Subcoercive Cyclic Electrical Loading of Lead Zirconate Titanate Ceramics I: Nonlinearities and Losses in the Converse Piezoelectric Effect, Journal of the American Ceramic Society 92, 2291 (2009).

[35] D. V. Taylor, D. Damjanovic, and N. Setter, Nonlinear contributions to dielectric and piezoelectric properties in lead zirconate titanate thin films, Ferroelectrics 224, 299 (1999).

[36] D. V. Taylor, D. Damjanovic, E. Colla, and N. Setter, Fatigue and nonlinear dielectric response in sol-gel derived lead zirconate titanate thin films, Ferroelectrics 225, 91 (1999).

[37] D. Damjanovic and D. V. Taylor, Contributions to the nonlinear dielectric and piezoelectric response of ferroelectric thin films and ceramics, Ferroelectrics 221, 137 (1999).

[38] N. Bassiri-Gharb, I. Fujii, E. Hong, S. Trolier-Mckinstry, D. V. Taylor, and D. Damjanovic, Domain wall contributions to the properties of piezoelectric thin films, Journal of Electroceramics 19, 47 (2007).

[39] N. Bassiri-Gharb, S. Trolier-McKinstry, and D. Damjanovic, Strain-modulated piezoelectric and electrostrictive nonlinearity in ferroelectric thin films without active ferroelastic domain walls, Journal of Applied Physics 110, 124104 (2011).

[40] N. Bassiri-Gharb, S. Trolier-McKinstry, and D. Damjanovic, Piezoelectric nonlinearity in ferroelectric thin films, Journal of Applied Physics 100, 044107 (2006). 
[41] S. Trolier-McKinstry, N. Bassiri Gharb, and D. Damjanovic, Piezoelectric nonlinearity due to motion of $180^{\circ}$ domain walls in ferroelectric materials at subcoercive fields: A dynamic poling model, Applied Physics Letters 88, 202901 (2006).

[42] B. Noheda, D. E. Cox, G. Shirane, S.-E. Park, L. E. Cross, and Z. Zhong, Polarization Rotation via a Monoclinic Phase in the Piezoelectric 92 \% $\mathrm{PbZ} n_{1 / 3}$ $\mathrm{Nb}_{2 / 3} \mathrm{O}_{3}-8 \% \mathrm{PbTi}_{3}$, Physical Review Letter 86, 3891 (2001).

[43] B. Noheda, Z. Zhong, D. E. Cox, G. Shirane, S.-E. Park, and P. Rehrig, Electric-field-induced phase transitions in rhombohedral $\mathrm{Pb}\left(Z_{n_{1 / 3}} \quad \mathrm{Nb} b_{2 / 3}\right)_{1-x}$ $\mathrm{Ti}_{x} \mathrm{O}_{3}$, Physical Review B 65, 224101 (2002).

[44] Y. Uesu, M. Matsuda, Y. Yamada, K. Fujishiro, D. Cox, B. Noheda, and G. Shirane, Symmetry of High-Piezoelectric Pb-Based Complex Perovskites at the Morphotropic Phase Boundary: I. Neutron Diffraction Study on $\mathrm{Pb}\left(\mathrm{Zn}_{1} / 3 \mathrm{Nb}_{2} / 3\right) \mathrm{O}_{3}-9 \% \mathrm{PbTiO}_{3}$, Journal of the Physical Society of Japan 71, 960 (2002), https://doi.org/10.1143/JPSJ.71.960 .

[45] B. Noheda and D. E. Cox, Bridging phases at the morphotropic boundaries of lead oxide solid solutions, Phase Transitions 79, 5 (2006), https://doi.org/10.1080/01411590500467262 .

[46] B. Noheda, D. E. Cox, G. Shirane, J. A. Gonzalo, L. E. Cross, and S.-E. Park, A monoclinic ferroelectric phase in the $\mathrm{Pb}\left(\mathrm{Zr}_{1-x} \mathrm{Ti} \mathrm{i}_{x}\right) \mathrm{O}_{3}$ solid solution, Applied Physics Letters 74, 2059 (1999), https://doi.org/10.1063/1.123756 .

[47] M. J. Haun, E. Furman, S. J. Jang, and L. E. Cross, Thermodynamic theory of the lead zirconate-titanate solid solution system, part V: Theoretical calculations, Ferroelectrics 99, 63 (1989).

[48] E. P. Houwman, K. Vergeer, G. Koster, and G. Rijnders, Functional Properties of Polydomain Ferroelectric Oxide Thin Films, in Correlated Functional Oxides (Springer, 2017) Book section Chapter 2, pp. 29-53.

[49] E. Houwman, K. Vergeer, G. Koster, and G. Rijnders, Modelling functional properties of ferroelectric oxide thin films with a three-domain structure, arXiv preprint arXiv:1901.10883 (2019).

[50] N. A. Pertsev, V. G. Kukhar, H. Kohlstedt, and R. Waser, Phase diagrams and physical properties of single domain epitaxial $\mathrm{PbZr}_{1-x} \mathrm{Ti}_{x} \mathrm{O}_{3}$ thin films, Physical Review B 67, 054107 (2003).

[51] B. Noheda, D. E. Cox, G. Shirane, R. Guo, B. Jones, and L. E. Cross, Stability of the monoclinic phase in the ferroelectric perovskite $\mathrm{PbZr}_{1-x} \mathrm{Ti}_{x} \mathrm{O}_{3}$, Physical Review B: Condensed Matter and Materials Physics 63, 014103 (2000).

[52] M. D. Nguyen, M. Dekkers, E. Houwman, R. Steenwelle, X. Wan, A. Roelofs, T. Schmitz-Kempen, and G. Rijnders, Misfit strain dependence of ferroelectric and piezoelectric properties of clamped (001) epitaxial $\mathrm{Pb}\left(\mathrm{Zr}_{0.52}, \mathrm{Ti}_{0.48}\right) \mathrm{O}_{3}$ thin films, Applied Physics Letters 99, 252904 (2011). 
[53] R. J. A. Steenwelle, Strain and composition effects in epitaxial PZT thin films, Phd thesis (2012).

[54] G. Robert, D. Damjanovic, and N. Setter, Piezoelectric hysteresis analysis and loss separation, Journal of Applied Physics 90, 4668 (2001). 



\section{6}

\section{Hysteresis, loss and nonlinearity in epitaxial $\mathrm{PbZr}_{0.55} \mathrm{Ti}_{0.45} \mathrm{O}_{3}$ films: Polarization rotation under a DC bias field}

The hysteresis, loss and nonlinearity of the strain and polarization response of an epitaxial $\mathrm{PbZr}_{0.55} \mathrm{Ti}_{0.45} \mathrm{O}_{3}$ film were experimentally investigated for non-switching $A C$ excitation fields at a DC bias field of $20 \mathrm{kV} \mathrm{cm}^{-1}$. The measured strain is hysteretic and linear with excitation amplitude, whereas the polarization is hysteretic and highly nonlinear over the investigated $70 \mathrm{~Hz}$ to $5 \mathrm{kHz}$ frequency range. Furthermore, compared to the case with zero bias, the effective piezoelectric coefficient that is extracted from the strain response is almost not changed for the investigated field range. In contrast, the loss tangent and nonlinearity of the polarization response are strongly reduced. The observations can not be explained by the commonly used Rayleigh model and its extensions, but are very well explained by the recently proposed polarization rotation model, by addition of a non-zero bias field term to the model. This model describes the film properties as the result of the nonlinear rotation of the polarization vector within the unit-cell in response to the applied field, which is accompanied with viscous interaction of the domains. These results demonstrate that the polarization rotation model can describe the film response in a broad range of excitation frequencies and amplitudes, that far exceed the applicability range of the Rayleigh model. 


\subsection{Introduction}

Hysteresis is a common challenge in the use of piezoelectric ceramics and thin films in applications such as sensors, actuators and energy harvesters. [1-8] Hysteresis can cause energy loss in energy harvesting devices or positioning inaccuracy in actuators. [9] Several studies have identified the viscous interaction of ferroelectric domains as the source of hysteresis and the associated dielectric/ferroelectric loss for sub-coercive field excitations. [10-14] However, this approach fails to explain the observed field amplitude dependence of the dielectric and piezoelectric loss tangents. For $\mathrm{PbZr}_{x} \mathrm{Ti}_{1-x} \mathrm{O}_{3}(\mathrm{PZT})$ ceramics and polycrystalline or chemical solution deposited (CSD) films, the amplitude and frequency dependent hysteresis behaviour can be described by adapting the Rayleigh model that has been used for the description of hysteresis in ferromagnets, by considering the Barkhausen jumps. [15-18] In ferroelectrics the Rayleigh model considers the stochastic interaction of domain walls (DWs) with defects as jumps of the DWs from one energetic minimum to another analogous to Barkhausen jumps. [19, 20] Because of its stochastic description, the Rayleigh model can not take into account the crystal symmetry of the material, that is expected to strongly influence the hysteresis, loss and nonlinearity since the functional properties of the material are dependent on the crystal symmetry and thus on the growth orientation of an epitaxial film.[21] The Rayleigh model assigns a single parameter to describe the combined effect of nonlinearity and loss as the source of hysteresis. Even though the nonlinear response of some ceramics and CSD films can be described by the odd harmonics predicted by this model, the even harmonics, that are also observed in some experiments, can not be explained. [22, 23]

In an earlier publication we have shown that for epitaxial $\mathrm{PbZr}_{0.55} \mathrm{Ti}_{0.45} \mathrm{O}_{3}$ films with a monoclinic crystal symmetry, the hysteresis, loss and nonlinearity of functional properties can very well be described by a model that considers the rotation of the polarization vector within the unit cell, and that is accompanied by a viscous interaction of the domains. [24] Moreover, with this so-called polarization rotation model, it is possible to differentiate between the nonlinearity of the response, that is caused by the nonlinear response of the angle of the polarization vector to the applied field, and the loss due to viscous interaction of the domains. In addition, the polarization rotation model predicts all experimentally observed even and odd harmonics.

As applications usually also require a non-zero DC bias, it is of importance to be able to describe the hysteresis and loss of the piezoelectric materials under this condition. Some papers using the Rayleigh model, also report on the case of non-zero field bias. In these reports, the applicability range of the Rayleigh model increases and the hysteresis decreases with increasing bias. [25-28] However, the Rayleigh model does not allow to make quantitative predictions on the change of the Rayleigh parameters with applied bias. Here we experimentally show that for a monoclinic, epitaxial $\mathrm{PbZr}_{0.55} \mathrm{Ti}_{0.45} \mathrm{O}_{3}$ film, an extension of the polarization rotation model can quantitatvely describe the hysteresis, loss and nonlinearity in the strain and polarization also for non-zero bias fields. 


\subsection{Polarization rotation model with applied bias}

The polarization rotation model considers the rotation of the polarization vector in the (110)-plane in a (001)-oriented monoclinic thin film under the influence of an electric field excitation.[24] The polarization, measured across the film in a parallel plate capacitor configuration, is then given by

$$
P=P_{S} \cos (\theta)
$$

with $P_{S}$ the length of the spontaneous polarization vector and $\theta$ the angle between the [001]-axis and the polarization vector. Using a grounded bottom electrode, a positive voltage on the top electrode corresponds to a downward-oriented electric field so that in a poled film the polarization angle is measured with respect to a top-to-bottom oriented film normal axis. In a fully relaxed unit cell at zero field, the polarization vector is in the body diagonal and $\theta$ equals $\theta_{0}=54.7^{\circ}$. Our film is under compressive, in-plane stress, resulting in a rotation of the polarization vector (in zero field) towards the out-of plane direction, and thus in a lower polarization angle that is defined as $\theta_{0, f}$. [24] (The subscript $f$ indicates that the sample is a clamped thin film.) The associated polarization value is the remanent polarization $P_{r}=P_{S} \cos \left(\theta_{0, f}\right)$ of the film. The application of a (positive) bias field in the [001]-direction leads to a further rotation of the angle $\theta$ towards the [001]-axis and is denoted by $\theta_{\mathrm{DC}, f}$. The polarization is then $P_{\mathrm{DC}}=P_{S} \cos \left(\theta_{\mathrm{DC}, f}\right)$. Under an additionally applied AC field in the [001]-direction, the angle of the polarization vector oscillates around $\theta_{\mathrm{DC}, f}$, causing the oscillation of the polarization value around $P_{\mathrm{DC}}$, and consequently stretching and contraction of the unit-cell, and thus an oscillating strain in the [001]-direction by the piezoelectric effect.

In the following, we first present the strain and polarization loops of the film in response to large electric field amplitudes far exceeding the coercive field. From these measurements the strain, polarization and polarization angle are described as a function of applied field in the non-switching range of the loop, i.e. in the field range commonly used in hysteresis measurements. From these descriptions, the nonlinearity and loss tangent of the strain and polarization during hysteresis measurements are predicted based on an extension of the polarization rotation model by taking into account a non-zero bias field. Here we only consider polarization rotation and no polarization switching. Therefore the magnitude of the AC and DC fields in the experiments are selected accordingly. Furthermore we assume that for the used magnitudes of the AC and DC fields the extension of the polarization vector is negligible. [24, 29, 30]

\subsubsection{Strain response}

Typical large signal strain-electric field (S-E) and polarization-electric field (P-E) loops are shown in Fig. $6.1 \mathrm{a}$ ) and b). For the hysteresis measurements we consider 

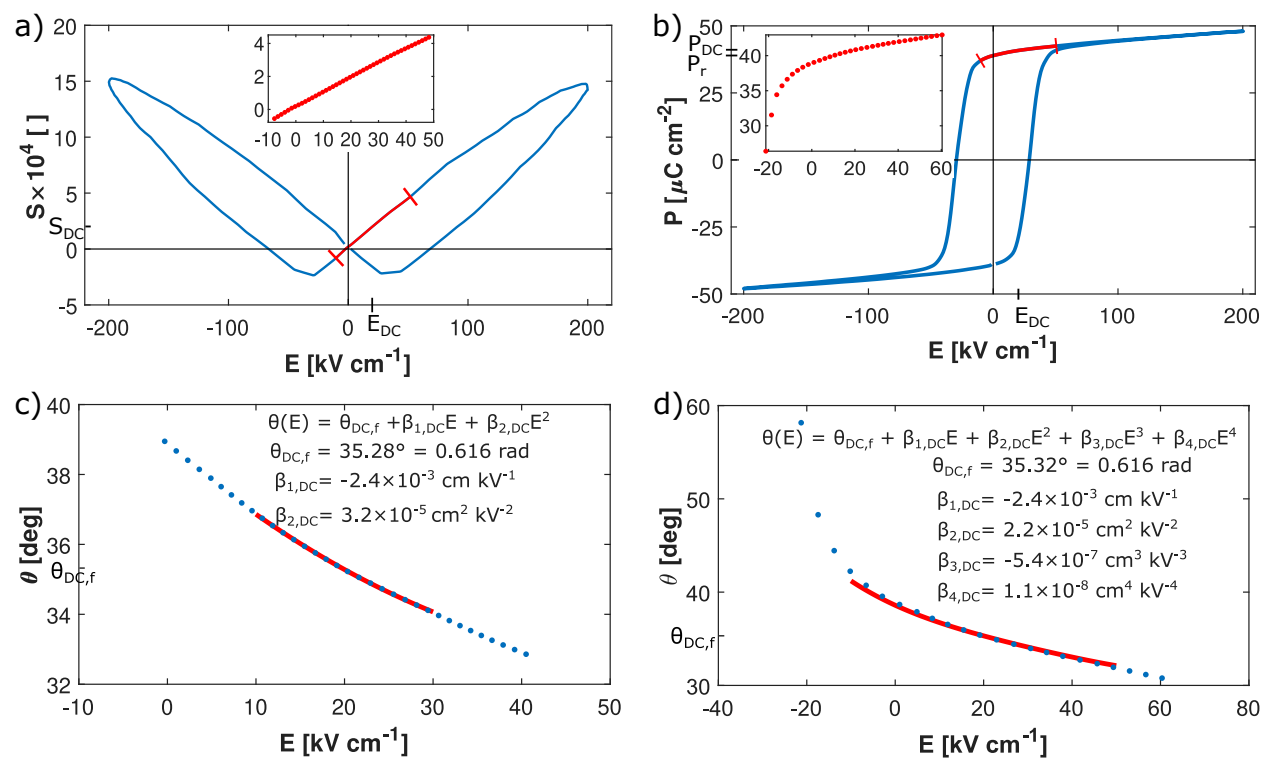

Figure 6.1: Global a) S-E and b) P-E loops. The red lines show the maximum trajectory of strain and polarization corresponding to $\mathrm{AC}$ cycling around the $20 \mathrm{kV} \mathrm{cm}^{-1} \mathrm{DC}$ bias. The insets show the magnified view of these trajectories. Field dependence of the polarization angle $\theta$ calculated from the inset in b) for AC field amplitudes of c) $10 \mathrm{kV} \mathrm{cm}^{-1}$ and d) $28 \mathrm{kV} \mathrm{cm}^{-1}$. The red lines give the fit of the datapoints with c) a second order polynomial in the 10 to $30 \mathrm{kV} \mathrm{cm}^{-1}$ field range and d) a fourth order polynomial in the -8 to $48 \mathrm{kV} \mathrm{cm}^{-1}$ field range. All measurements are at $3 \mathrm{kHz}$.

a downward polarized sample driven by an AC field excitation on top of a zero or positive field bias, so that the polarization (strain) oscillates along the falling branch of the large signal S-E (P-E) loop. A positive DC bias of $20 \mathrm{kV} \mathrm{cm}^{-1}$ during the hysteresis measurements causes the central point of the AC field to shift along the field x-axis. The strain and polarization values shift accordingly on the global S-E and P-E loops as shown with red lines in Fig. 6.1 a) and b). The measured strain around the bias field is in good approximation $\left(R^{2}=0.9994\right)$ linear with the applied field, as shown in the inset. We can therefore describe the DC strain by the linear relation $S_{\mathrm{DC}}=d_{33, f} E_{\mathrm{DC}}$, where $d_{33, f}$ and $E_{\mathrm{DC}}$ are respectively the effective piezoelectric coefficient in the out-of-plane direction of the clamped thin film and the applied DC bias field. One can then also describe the AC strain response by a linear relation that is in-phase $(i p)$ with the applied AC field $E(t)$, thus $S_{\mathrm{ip}}(t)=d_{33, f} E(t)$ for oscillating fields that do not extend beyond the subcoercive field, similar as was done for the zero bias case. The validity of the linear dependence can in good approximation be derived from the Landau-Devonshire model. [24] The observation of elliptic strain hysteresis loops in our experiments suggests that the piezoelectric loss is dominated by a viscous loss mechanism. [24] The viscous loss can be introduced as an out-of-phase (oop) component in the strain response which is proportional to the time derivative of the in-phase response as $S_{\text {oop }}(t)=\gamma_{S} \dot{S_{\text {ip }}}(t)$, where $\gamma_{S}$ is a viscosity coefficient. The total strain is then the 
sum of $S_{\mathrm{DC}}, S_{\mathrm{ip}}$ and $S_{\mathrm{oop}}$

$$
S(E)=d_{33, f} E_{\mathrm{DC}}+d_{33, f} E+\gamma_{S} d_{33, f} \dot{E}
$$

One arrives at a simple expression for the strain response to a small amplitude, sinusoidal field, $E(t)=E_{0} \sin (\omega t)$, similar to that for the zero bias case, apart from the $S_{\mathrm{DC}}$ offset.

$$
S(t)=d_{33, f} E_{\mathrm{DC}}+d_{33, f} E_{0} \sin (\omega t)+\gamma_{d} \omega E_{0} \cos (\omega t)
$$

Here we defined $\gamma_{d}=\gamma_{S} d_{33, f}$. It is noted that in the following experiments use is made of a double beam laser interferometer (DBLI), that allows only the determination of strain changes, hence the value of $S_{\mathrm{DC}}$ can not be determined. This however does not change the validity of the model, as the DC component of the strain only works as an offset and does not influence the scaling of loss or hysteresis. As a consequence of the assumed viscous nature of the loss process the unit cell deformation is lagging in time in response to varying fields, thus one expects that $\gamma_{d}<0$. We do not exclude the presence of any implicit frequency dependencies of the parameters $d_{33, f}$ and $\gamma_{d}$, which could stem from frequency dependent viscous interactions. However the nature of such interactions is poorly understood and described in literature. Instead we will therefore extract the frequency dependence of these parameters from the hysteresis measurements at different frequencies.

The strain loss, or loss tangent, is simply given by the ratio of the viscous to non-viscous components of the strain:

$$
\tan \delta_{S}=\left|\gamma_{d} \omega / d_{33, f}\right|=\left|\gamma_{S} \omega\right|
$$

\subsubsection{Polarization response}

A typical global P-E loop is shown in Fig. 6.1 b). For the hysteresis measurements we consider only the decreasing field branch, because the film is poled downwards before each measurement. The application of a positive DC field bias of $20 \mathrm{kV} \mathrm{cm}^{-1}$ leads to a shift of the center point of the $\mathrm{AC}$ polarization response to the value $P=P_{\mathrm{DC}}$. The hysteresis measurements have been performed with two AC field amplitudes $\left(10 \mathrm{kV} \mathrm{cm}^{-1}\right.$ and $\left.28 \mathrm{kV} \mathrm{cm}^{-1}\right)$. The lower amplitude is the same as was used for previous experiments without DC bias in Lucke et.al. [24]. The large amplitude was chosen because it is the largest amplitude that can be used without observable effects of polarization switching on the hysteresis. This amplitude results in an oscillation of the electric field from -8 to $48 \mathrm{kV} \mathrm{cm}^{-1}$ that enables to explore the validity of the polarization rotation model in a field range that is far exceeding the Rayleigh range. The red line in Fig. $6.1 \mathrm{~b}$ ) shows the maximum range of field and polarization change for the second $\mathrm{AC}$ amplitude.

The change of the polarization angle $\theta$ around $\theta_{\mathrm{DC}, f}$, that is in-phase with the driving field is described by $\theta_{\mathrm{ip}}=\Delta \theta(E(t))$. The out-of-phase part is described as $\theta_{\text {oop }}=\gamma_{P} \Delta \dot{\theta}$, assuming a linear viscous interaction with viscousity coefficient $\gamma_{P}$ 
to be present. The change of the polarization angle is then given by the summation of the three contributions.

$$
\theta(E)=\theta_{\mathrm{DC}, f}+\Delta \theta(E)+\gamma_{P} \Delta \dot{\theta}(E)
$$

The change of the polarization angle that is in-phase with the applied field can be described with a polynomial that has the general form:

$$
\Delta \theta(E)=\beta_{1, \mathrm{DC}} E+\beta_{2, \mathrm{DC}} E^{2}+\beta_{3, \mathrm{DC}} E^{3} \ldots=\sum_{i=1}^{p} \beta_{i, \mathrm{DC}} E^{i}
$$

where $p$ is the order of the polynomial. The value of $p$ in the first place depends on the $\mathrm{AC}$ amplitude $E_{0}$, but also on the strain state of the film, consequently on the substrate, the deposition temperature and the film composition, since these change the curvature of the P-E loop. Because the polarization angle decreases with increasing field, the odd $\beta$ coefficients should be negative. The even $\beta$ coefficients give rise to a possible asymmetry in the field sensitivity of $\Delta \theta(E)$ around $\theta_{\mathrm{DC}, f}$. Furthermore, the viscosity parameter $\gamma_{P}$ is expected to be negative as the out-ofphase response should lag behind the electric field.

Fig. $6.1 \mathrm{c})$ and $\mathrm{d}$ ) give the angle $\theta(E)$ that is calculated from the global P-E loop using Eq. 6.1. For both AC amplitudes the curves are fitted with a polynomial of order two $\left(R^{2}=0.9999\right)$ and four $\left(R^{2}=0.9997\right)$, respectively. These are the lowest order polynomials that can accurately describe the nonlinearity of the measured $\theta(E)$. The fit values of the polynomial coefficients, that will be used further on in this study, are given in Fig. 6.1c) and d). It is seen that the expectations with respect to the signs of the coefficients match with the signs of the fitted values. Further, the increase in the AC field amplitude just leads to an increase of the order of the polynomial $p$, because the covered part of the $\theta(E)$-curve becomes increasingly curved, but the $\beta_{i}$ parameters remain of the same order of magnitude for different AC amplitudes.

A comparison of the fitted polynomials for $\mathrm{AC}$ amplitude of $10 \mathrm{kV} \mathrm{cm}^{-1}$ with and without DC bias, shows that the order of the polynomial decreases from 4 to 2 for the non-zero bias case. [24] Moreover, with zero bias the values of the $\beta$ coefficients $\left(\beta_{1}=-3.9 \times 10^{-3} \mathrm{~cm} \mathrm{kV}^{-1}\right.$ and $\beta_{2}=1.1 \times 10^{-4} \mathrm{~cm} \mathrm{kV}^{-2}$ [24]) are significantly larger than the corresponding $\beta_{i, \mathrm{DC}}$-coefficients in Figure $6.1 \mathrm{c}$ ), because of the increased average slope and asymmetry of the P-E loop for zero bias

Inserting Eq. 6.5 and Eq. 6.6 into Eq. 6.1 and using some trigonometric identities, the dependence of the polarization on the angular change $\theta(E)$ with applied DC bias can be written as:

$$
\begin{aligned}
P(\theta(E)) & =P_{S} \cos \left(\theta_{\mathrm{DC}, f}+\Delta \theta+\gamma_{P} \Delta \dot{\theta}\right) \\
& =P_{\mathrm{DC}}\left[\cos \left(\Delta \theta+\gamma_{P} \Delta \dot{\theta}\right)-\tan \left(\theta_{\mathrm{DC}, f}\right) \sin \left(\Delta \theta+\gamma_{P} \Delta \dot{\theta}\right)\right]
\end{aligned}
$$


Here $P_{\mathrm{DC}}=P_{S} \cos \left(\theta_{\mathrm{DC}, f}\right)$. From Fig. $\left.6.1 \mathrm{c}\right)$ and d) it can be seen that $\Delta \theta(E)$ is of the order of a few degrees allowing the use of lowest order Taylor expansions of the sin and cos functions. This approximation results in a nonlinear, analytically tractable, polynomial expression for the time-dependent polarization response, which can be written concisely as a sum of sine and cosine functions, that depends on the order $p$ of the $\Delta \theta(E)$ polynomial:

$$
P(t)=P_{\mathrm{DC}}\left[c_{0}+\sum_{n=1}^{2 p} c_{n, s} E_{0}^{n} \sin (n \omega t)+c_{n, c} E_{0}^{n} \cos (n \omega t)\right]
$$

The coefficients of the harmonics $c_{0}, c_{n, s}$ and $c_{n, c}$ are functions of the polynomial parameters $\beta_{i, \mathrm{DC}}$, viscosity coefficient $\gamma_{P}$, the AC field frequency $\omega$ and the $\mathrm{AC}$ field amplitude $E_{0}$. In the Supporting Information B.1 explicit forms of these functions are given. Note that all coefficients are even functions of $E_{0}$ and that the amplitude of the harmonics rapidly decreases with increasing harmonic number $n$. The $\sin (n \omega t)$ terms give rise to peaks in the frequency domain at all frequencies $n \omega$, with $n=1$ being the fundamental harmonic and $n>1$ being the corresponding higher harmonics. Thus the model predicts that in the polarization signal all harmonics are present up to fourth and eight order for $E_{0}=10 \mathrm{kV} \mathrm{cm}^{-1}$ and $28 \mathrm{kV} \mathrm{cm}^{-1}$, respectively. Furthermore, hysteresis is expected to be present in all harmonics, since for every harmonic there is a non-zero cosine term in the expansion.

It is found from calculation using the determined coefficients that $c_{0} \approx 1$ for all used amplitudes and frequencies. Consequently the first and higher order hysteresis loops are all centered around the same bias point $P_{\mathrm{DC}}$. The amplitude of the $n^{\text {th }}$ polarization harmonic in the case of applied bias can be calculated by:

$$
\left|P_{n, \mathrm{DC}}\left(E_{0}\right)\right|=P_{\mathrm{DC}}\left(E_{0}^{n} \sqrt{c_{n, s}^{2}+c_{n, c}^{2}}\right)
$$

One can now predict the scaling of $\left|P_{n, \mathrm{DC}}\left(E_{0}\right)\right|$ with the AC field amplitude from the $E_{0}$ dependence of the $c$-coefficients. For $E_{0} \leq 10 \mathrm{kV} \mathrm{cm}^{-1}$ the harmonic amplitudes are predicted to scale as:

$$
\begin{aligned}
& \left|P_{1, \mathrm{DC}}\left(E_{0}\right)\right|=P_{\mathrm{DC}}\left(k_{1,1} E_{0}+k_{1,3} E_{0}^{3}\right) \\
& \left|P_{2, \mathrm{DC}}\left(E_{0}\right)\right|=P_{\mathrm{DC}}\left(k_{2,2} E_{0}^{2}+k_{2,4} E_{0}^{4}\right) \\
& \left|P_{3, \mathrm{DC}}\left(E_{0}\right)\right|=P_{\mathrm{DC}}\left(k_{3,3} E_{0}^{3}\right) \\
& \left|P_{4, \mathrm{DC}}\left(E_{0}\right)\right|=P_{\mathrm{DC}}\left(k_{4,4} E_{0}^{4}\right)
\end{aligned}
$$

The $k_{n, m}$ coefficients are new functions of the $\beta$-coefficients and $\omega$, where $n$ is again the order of the harmonic and $m$ is the power of the corresponding field amplitude term. The predicted scaling of the polarization amplitude for $E_{0} \leq 10 \mathrm{kV} \mathrm{cm}^{-1}$ with $E_{\mathrm{DC}}=20 \mathrm{kV} \mathrm{cm}^{-1}$ bias is less nonlinear than for the same AC amplitude with zero bias, as the model predicts additional $5^{\text {th }}$ and $6^{\text {th }}$ order harmonics for the latter 
case and only up to $4^{\text {th }}$ order for the bias case.

For $E_{0} \leq 28 \mathrm{kV} \mathrm{cm}^{-1}$ the harmonic amplitudes are predicted to scale as:

$$
\begin{aligned}
\left|P_{1, \mathrm{DC}}\left(E_{0}\right)\right| & =P_{\mathrm{DC}}\left(k_{1,1} E_{0}+k_{1,3} E_{0}^{3}+k_{1,5} E_{0}^{5}\right) \\
\left|P_{2, D C}\left(E_{0}\right)\right| & =P_{D C}\left(k_{2,2} E_{0}^{2}+k_{2,4} E_{0}^{4}+k_{2,6} E_{0}^{6}\right) \\
\left|P_{3, D C}\left(E_{0}\right)\right| & =P_{D C}\left(k_{3,3} E_{0}^{3}+\left(k_{3,5} E_{0}^{5}\right)\right. \\
\left|P_{4, D C}\left(E_{0}\right)\right| & =P_{D C}\left(k_{4,4} E_{0}^{4}+k_{4,6} E_{0}^{6}\right) \\
\left|P_{5, D C}\left(E_{0}\right)\right| & =P_{D C}\left(k_{5,5} E_{0}^{5}\right) \\
\left|P_{6, D C}\left(E_{0}\right)\right| & =P_{D C}\left(k_{6,6} E_{0}^{6}\right) \\
\left|P_{7, D C}\left(E_{0}\right)\right| & =P_{D C}\left(k_{7,7} E_{0}^{7}\right) \\
\left|P_{8, D C}\left(E_{0}\right)\right| & =P_{D C}\left(k_{8,8} E_{0}^{8}\right)
\end{aligned}
$$

There is a clear increasing nonlinear dependence of the polarization response on $E_{0}$ because of the presence of higher order polynomial terms and an increase of the number of harmonics. Overall, the amplitude of the harmonics scale either with even or odd powers of the field amplitude, depending on the parity of the order of the harmonic. This relation was also found for the zero bias case. [24]

The energy loss per polarization hysteresis cycle is proportional to the area enclosed by the hysteresis loop in the field domain, $\int_{\text {cycle }} P(E) d E$. It appears that only the fundamental harmonic has a non-zero area as the areas of all higher harmonics are described by integrals of the product of an even and an odd function and is as such zero. (See Supporting Information B.2 for further details). The polarization loss tangent $\tan \delta_{P}$, can therefore be calculated simply as the ratio of the coefficients of the viscous and non-viscous part of the fundamental harmonic:

$$
\tan \delta_{P}=\left|\frac{c_{1, c}}{c_{1, s}}\right| \approx\left|\gamma_{P} \omega\right| .
$$

It is easy to show that the last approximation step holds by substituting the numerical values in the expressions of $c_{1, c}$ and $c_{1, s}$. This is the same expression as for the strain loss tangent and for the zero bias case. [24]

\subsection{Experimental details}

The film used in the experiment was a $3 \mu \mathrm{m}$ thick monoclinic $\mathrm{PbZr}_{0.55} \mathrm{Ti}_{0.45} \mathrm{O}_{3}$ film, sandwiched between $100 \mathrm{~nm}$ thick $\mathrm{LaNiO}_{3}$ electrodes on a (001)-oriented single terminated $\mathrm{SrTiO}_{3}$ substrate. For details on the film growth and crystallographic characterisation see Lucke et. al. [24] The strain and polarization were measured with a double beam laser interferometer (aixDBLI) combined with the aixACCT TF-2000 Analyzer. For the hysteresis measurements an oscillatory field with variable frequency in the range $f=70 \mathrm{~Hz}$ to $5 \mathrm{kHz}$ and amplitude $E_{0}$ in the range 2.5 to $28 \mathrm{kV} \mathrm{cm}^{-1}$ was used on top of a DC bias field of $20 \mathrm{kV} \mathrm{cm}^{-1}$. This bias field was chosen to be equal to the coercive field at $1 \mathrm{kHz}$ measurement frequency. In 
the hysteresis experiments a maximum AC field amplitude of $28 \mathrm{kV} \mathrm{cm}^{-1}$ was chosen, so that even for the largest AC field amplitude due to the bias field one stays away safely from the coercive field at $-20 \mathrm{kV} \mathrm{cm}^{-1}$ on the decreasing branch of the polarization loop.

\subsection{Results and discussion}

Figure 6.2 shows the Fourier Transform of the measured strain and polarization signal in response to a sinusoidal driving field at $3 \mathrm{kHz}$. The left and right columns show the signals for $E_{0}=10 \mathrm{kV} \mathrm{cm}^{-1}$ and $28 \mathrm{kV} \mathrm{cm}^{-1}$ respectively, for a bias field of $E_{D C}=20 \mathrm{kV} \mathrm{cm}^{-1}$. The raw strain data in Fig. $6.2 \mathrm{a}$ ) and b) show only a single peak at the fundamental frequency of $3 \mathrm{kHz}$. No higher harmonics can be identified. (Some high amplitude noise peaks appear at higher frequencies, but we attribute these to numerical artifacts of the Fast Fourier Transform algorithm, because of their very narrow peak width.) Below $2 \mathrm{kHz}$ one can clearly observe a frequency dependent noise that is inversely proportional to the frequency. The polarization signal, Fig. 6.2 c) and d), shows several higher harmonics superimposed on the frequency dependent background noise. For small and large $E_{0}$ respectively all harmonics up to $3^{r d}$ and $9^{t h}$ order are observed in agreement with the predictions of the the polarization rotation model in Eq. 6.10 and Eq. 6.11. Not observing the predicted $4^{\text {th }}$ order harmonic for the low $E_{0}$ case can be explained by the rapidly declining amplitude of the harmonics, such that the $4^{\text {th }}$ harmonic is hidden in the noise, see Supporting Information B.1.1, Tab. B.1 and Fig. B.1. The observation of a $9^{t h}$ order harmonic for the high $E_{0}$ case when the model predicts only harmonics up to $8^{t h}$ order is related to the choice of the lowest order polynomial, a higher order polynomial would lead to higher orders, but would also make the equations too complicated to follow. The observed frequency dependent noise was fitted with a power law dependence $P_{\text {noise }}(f)=a f^{b}$ in the polarization data where the frequency dependent noise is more apparent. The exponent was obtained as $b=-0.89 \pm$ 0.03 by taking into account all the polarization measurements, indicating the $1 / f$ character of the noise. The obtained $1 / f$ baseline is also overlaid to the strain signal below the fundamental frequency. The $1 / f$ baseline closely matches the observed frequency dependent baseline for both the polarization and the strain in Fig. 6.2.

\subsubsection{Strain hysteresis measurements}

The low frequency noise in the strain data hinders an accurate fit of the model to the raw measurement data. For this reason the strain signal was band-pass filtered around the fundamental harmonic and then averaged over the measured 20 cycles. [24] In Fig. 6.3 the strain signals (markers) and the fits using Eq. 6.3 (lines) are shown for four amplitudes. For clarity only every fifth data point is shown. The standard deviation of the filtered and averaged data points with respect to the fit is smaller than the size of the markers. The strain hysteresis loops in Fig. 6.3 b) are elliptical in contrast to the lenticular shapes expected from the Rayleigh model. The data and fits for the other frequencies are shown in Supporting Information B.3. For all amplitudes and frequencies, the linear, viscous strain model accurately 

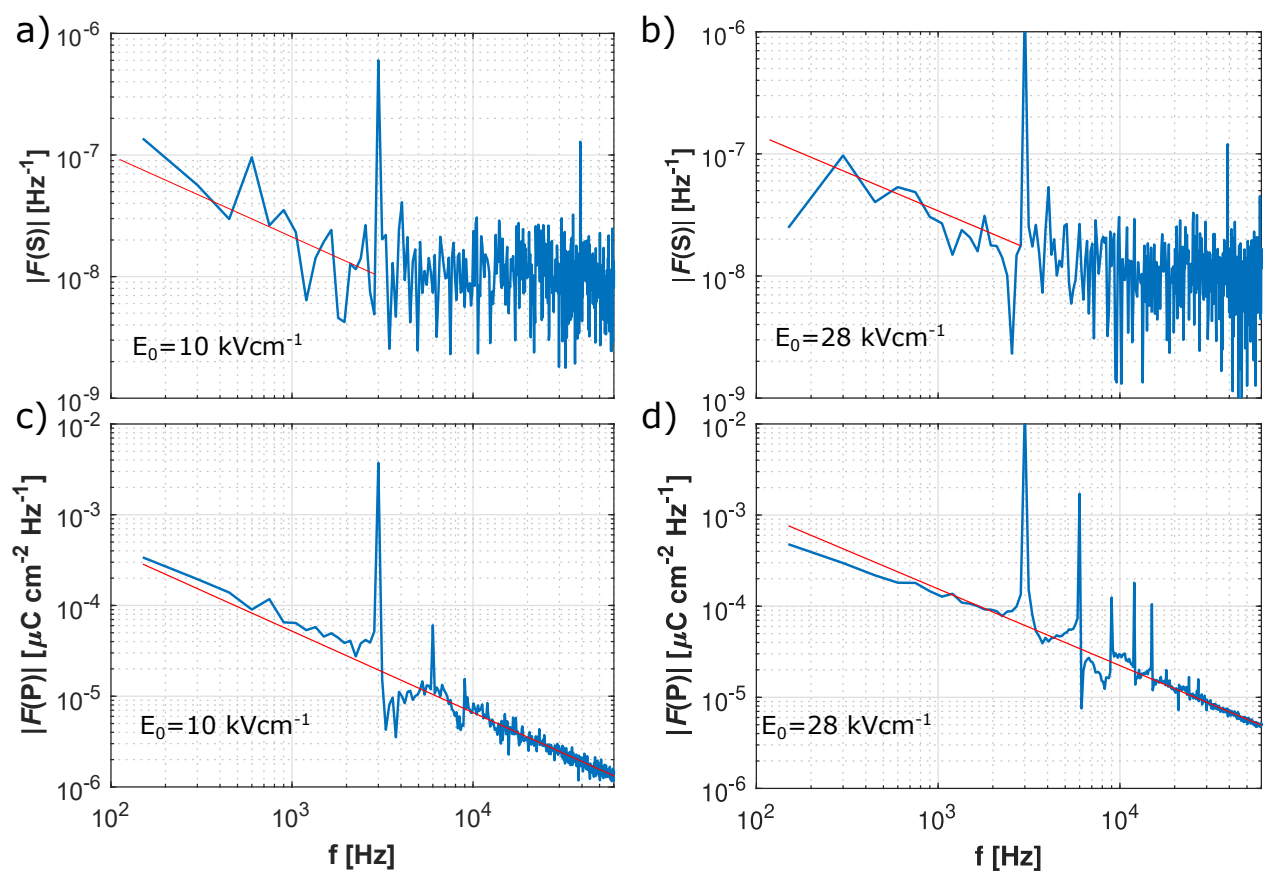

Figure 6.2: Fourier transform of $a, b)$ the measured strain and $c, d)$ the polarization signal for a sinusoidal signal of 20 cycles at $3 \mathrm{kHz}$. Respectively, left and right columns show the signal for AC field amplitudes of $10 \mathrm{kV} \mathrm{cm}^{-1}$ and $28 \mathrm{kV} \mathrm{cm}^{-1}$. The red line indicates the fitted $1 / f$ noise baseline.

fits the data.
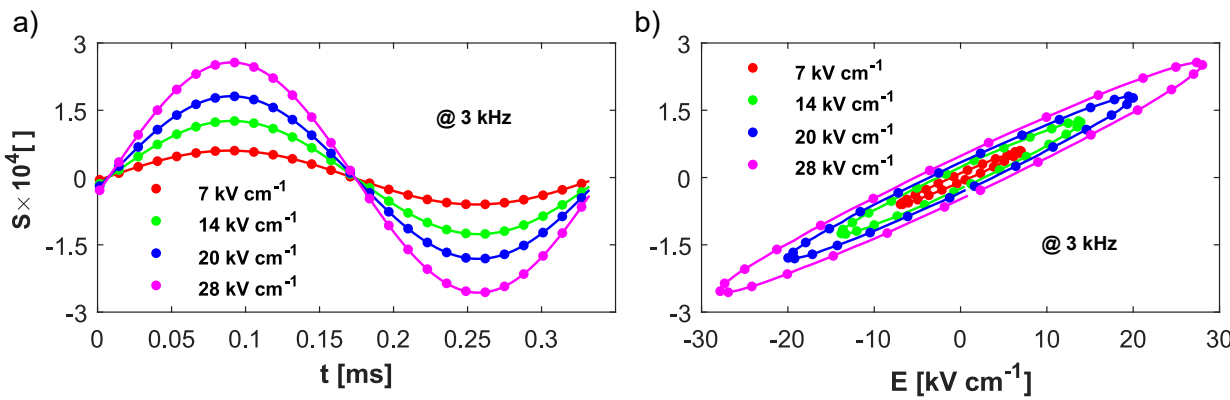

Figure 6.3: Filtered and averaged strain signal as function of a) time and b) applied cyclic field, $E(t)$. Datapoints show the measurement and the lines are obtained from fitting with Eq. 6.3.

The extracted model parameters $d_{33, f}\left(E_{0}, f\right)$ and $\gamma_{d}\left(E_{0}, f\right)$ at $3 \mathrm{kHz}$ frequency are plotted as a function of the field amplitude $E_{0}$ in Fig. $6.4 \mathrm{a}$ ) and b). The statistical uncertainty of the fitted parameters is smaller than the size of the markers. One observes no obvious field dependence of $d_{33, f}$ and $\gamma_{d}$. Both model parameters fluc- 
tuate around an average value, i.e. the average of the model parameters at different $E_{0}$, that can be represented by $\left\langle d_{33, f}\right\rangle_{E_{0}}(3 \mathrm{kHz})$ and $\left\langle\gamma_{d}\right\rangle_{E_{0}}(3 \mathrm{kHz})$, and indicated by the solid lines in the figure. The shaded areas indicate the $\pm 2 \sigma$ confidence intervals of the averages. The average values are $\left\langle d_{33, f}\right\rangle_{E_{0}}(3 \mathrm{kHz})=88 \mathrm{pm} \mathrm{V}^{-1}$ and $\left\langle\gamma_{d}\right\rangle_{E_{0}}(3 \mathrm{kHz})=-9 \times 10^{-11} \mathrm{~cm} \mathrm{kV}^{-1} \mathrm{~Hz}^{-1} \mathrm{rad}^{-1}$.

Fig. 6.4c) shows the dependence of the loss tangent on the excitation field amplitude. The datapoints are calculated from the area of the hysteresis loop measured for that $E_{0}$ value. The lines correspond to the loss tangent calculated using Eq. 6.4 with the field averaged values $\left\langle d_{33, f}\right\rangle_{E_{0}}(3 \mathrm{kHz})$ and $\left\langle\gamma_{d}\right\rangle_{E_{0}}(3 \mathrm{kHz})$. The confidence intervals (shaded areas) are calculated using the error propagation of the confidence interval of these parameters. The measured loss tangent data shows much less spread than the $\pm 2 \sigma$ confidence interval, except for those at the lowest $E_{0}$ values. We conclude that within the measurement accuracy the loss tangent is independent of the excitation amplitude, as is expected on the basis of the model if $d_{33, f}$ and $\gamma_{d}$ are independent of the field amplitude. Experimentally we also find that $d_{33, f}$, $\gamma_{d}$ and $\tan \delta_{S}$, within the investigated amplitude range, are independent of the AC field amplitude. This also implies that the global S-E loop in the -8 to $48 \mathrm{kV} \mathrm{cm}^{-1}$ range is linear, as was already observed in Fig. 6.1 a). The data for the zero bias case is plotted in blue in Fig. 6.4 for comparison with the $20 \mathrm{kV} \mathrm{cm}^{-1}$ bias case. We observe that the application of a bias field has no significant effect on the values of the strain parameters. It is seen that the average $\left\langle d_{33, f}\right\rangle_{E_{0}}(3 \mathrm{kHz})$ decreases from $96 \mathrm{pm} \mathrm{V}^{-1}$ for the zero bias case to $88 \mathrm{pm} \mathrm{V}^{-1}$ for the bias case, which means that the average slope in the range -10 to $10 \mathrm{kV} \mathrm{cm}^{-1}$ is slightly larger than in the range -8 to $48 \mathrm{kV} \mathrm{cm}^{-1}$. We do not attribute any significance to the small difference in average $\gamma_{d}$ and $\tan \delta_{S}$ values since the differences are within the confidence intervals.

In Fig. 6.5 a) and b) the averaged values $\left\langle d_{33, f}\right\rangle_{E_{0}}$ and $\left\langle\gamma_{d}\right\rangle_{E_{0}}$ are shown as a function of the excitation frequency. The error bars denote the standard deviation of these parameters over the investigated field range for that frequency. Orange and blue colors are used for the bias and zero bias case, respectively. It is seen that $\left\langle d_{33, f}\right\rangle_{E_{0}}$ and $\left\langle\gamma_{d}\right\rangle_{E_{0}}$ are also independent of frequency. The field and frequency averaged values, $\left\langle d_{33, f}\right\rangle_{E_{0}, \omega}$ and $\left\langle\gamma_{d}\right\rangle_{E_{0}, \omega}$, and the corresponding confidence intervals are denoted by the horizontal lines and the shaded areas, respectively. The field and frequency averaged value of the piezoelectric coefficient is $\left\langle d_{33, f}\right\rangle_{E_{0}, \omega}=90 \mathrm{pm} \mathrm{V}^{-1}$. For $\left\langle\gamma_{d}\right\rangle_{E_{0}}$ the low frequency data show larger error bars, which is attributed to the large $1 / f$-noise at these frequencies. The field and frequency averaged value is $\left\langle\gamma_{d}\right\rangle_{E_{0}, \omega}=-10 \times 10^{-11} \mathrm{~cm} \mathrm{kV}^{-1} \mathrm{~Hz}^{-1} \mathrm{rad}^{-1}$, so that the viscosity coefficient for strain is obtained as $\left\langle\gamma_{S}\right\rangle_{E_{0}, \omega}=-1.1 \times 10^{-5} \mathrm{~Hz}^{-1} \mathrm{rad}^{-1}$.

In Fig. $6.5 \mathrm{c}$ ) the field averaged loss tangent values are shown as a function of the frequency. Again the loss tangent is calculated from the area of the hysteresis loops and the error bars denote the standard deviation of the data for different $E_{0}$-values. The lines are calculated with Eq. 6.4 using the field and frequency averaged values $\left\langle d_{33, f}\right\rangle_{E_{0}, \omega}$ and $\left\langle\gamma_{d}\right\rangle_{E_{0}, \omega}$. The $\pm 2 \sigma$ confidence intervals are calculated from the error propagation of the $\pm 2 \sigma$ confidence intervals in Fig. $6.5 \mathrm{a}$ ) and b). It is observed 

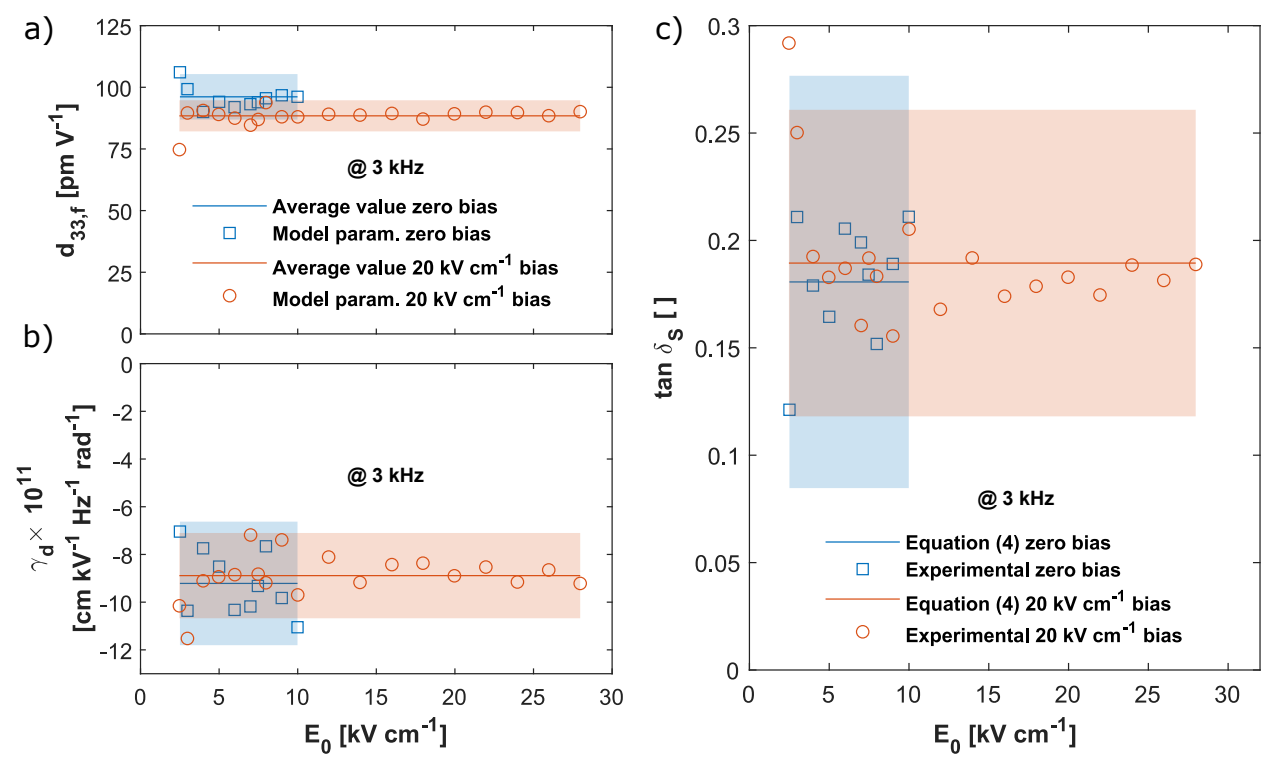

Figure 6.4: Field amplitude dependence of the parameters a) $d_{33, f}$ and b) $\gamma_{d}$ for zero bias (blue) and $20 \mathrm{kV} \mathrm{cm}^{-1}$ bias (orange). The lines denote the average value $\left\langle d_{33, f}\right\rangle_{E_{0}}$ and $\left\langle\gamma_{d}\right\rangle_{E_{0}}$ and the shaded areas indicate the $\pm 2 \sigma$ confidence intervals. c) $\tan \delta_{S}$ calculated from the area of the hysteresis loops. The lines and confidence intervals are calculated with Eq. 6.4 using the data in a) and b).

that the experimental loss tangent scales linearly with frequency, as expected from Eq. 6.4, which supports the assumption of a viscous loss mechanism for the strain.

Our results clearly show that the model parameters $d_{33, f}, \gamma_{d}$ and thus $\gamma_{S}$ are independent of the excitation field amplitude and frequency. We observe furthermore, that the application of a bias field does not lead to a significant change of the values of these parameters and the loss tangent. As already remarked previously, the Rayleigh model cannot explain the observed frequency and amplitude dependencies of the loss tangent, while these follow naturally from our model, assuming a frequency and amplitude independent viscosity constant $\gamma_{S}$. 

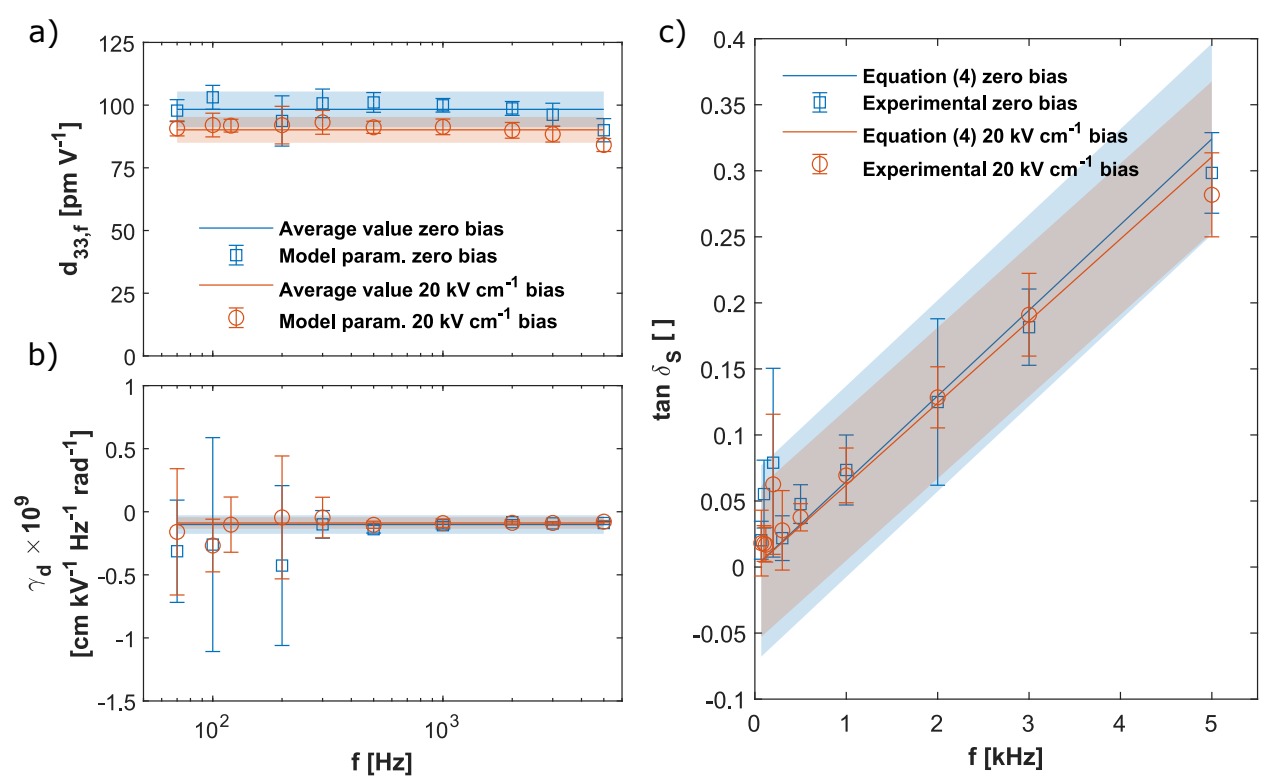

Figure 6.5: a) Average piezoelectric coefficient $\left\langle d_{33, f}\right\rangle_{E_{0}}$ and b) viscosity parameter $\left\langle\gamma_{d}\right\rangle_{E_{0}}$ for zero bias (blue) and $20 \mathrm{kV} \mathrm{cm}^{-1}$ bias (orange). The error bars denote the standard deviation of the field averaged values. The lines and shaded areas indicate the averages $\left(\left\langle d_{33, f}\right\rangle_{E_{0}, \omega}\right.$ and $\left.\left\langle\gamma_{d},\right\rangle_{E_{0}, \omega}\right)$ and their $\pm 2 \sigma$ confidence intervals, respectively. c) Loss tangent data points are calculated from the area of the hysteresis loops. The lines and shaded confidence intervals are calculated from the average values in a) and b) using Eq. 6.4.

\subsubsection{Polarization hysteresis measurements}

The amplitudes of the first and higher harmonics (up to $5^{\text {th }}$ harmonic) of the polarization signal are plotted as function of the field amplitude $E_{0}$ in Fig. 6.6. For harmonics with $n>5$ the amplitude is below the $1 / f$-noise baseline, except for the largest value of $E_{0}$. The lines are fits for the zero bias (blue) and $20 \mathrm{kV} \mathrm{cm}^{-1}$ bias case (orange), using Eq. 6.11. The only constraint imposed on the fits is that the $k_{n, m}$ coefficients are positive as is expected from the model. Clearly the fitted polynomials describe accurately the experimentally observed field dependencies, demonstrating the applicability of the model. Fig. 6.6 shows that the $P_{n}$ for all harmonics at the zero bias case increases much rapidly with increasing field amplitude compared to the non-zero bias case.

It is noted that it is not possible to describe the amplitudes of the harmonics in terms of the determined $\beta$-coefficients because the harmonic peaks in the measured Fourier spectra show considerable frequency spreading, implying significant energy transfer from the harmonic frequency to the neighbouring frequencies. This mechanism is not taken into account in the model and causes a discrepancy between the theoretical and experimental values of the $c_{n, s / c}$ coefficients and thus of the $k_{n, m}$ coefficients. Nevertheless, as was already concluded for the case of zero bias the Rayleigh model cannot explain the presence of all even and odd harmonics of the polarization hysteresis. Furthermore the third and higher order odd harmonics are 
clearly not scaling with $E_{0}^{2}$ as predicted by the Rayleigh model, but with higher order $E_{0}^{m}$ dependencies.
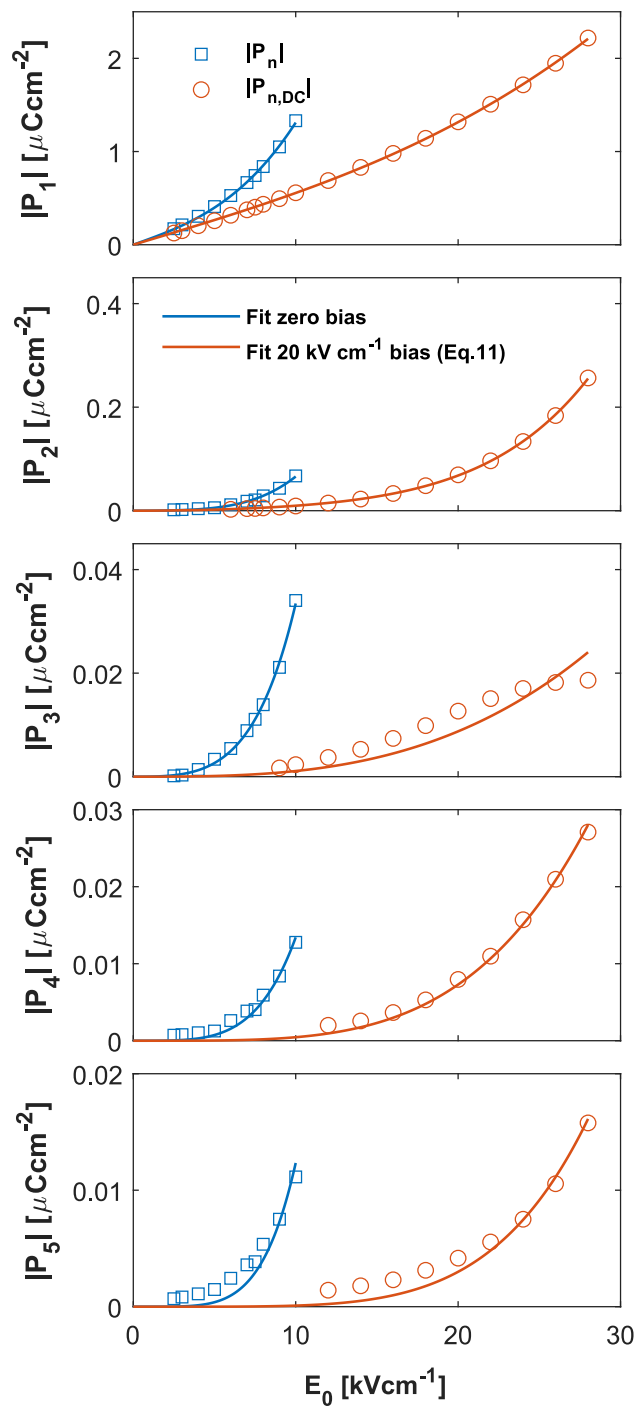

Figure 6.6: Scaling of the amplitude of the polarization harmonics with the field amplitude $E_{0}$. The data points are obtained from Fourier spectra of the polarization signal, such as shown in Figure 6.2 and the lines are fits to Eq. 6.11. The orange and blue colors represent the bias and zero bias case, respectively.

The loss behaviour, as described by the polarization rotation model, is determined by the fundamental harmonic only, Eq. 6.12. In good approximation the polarization response can be described by the fundamental harmonic only, because the $c_{n, s / c}$ coefficients for the higher harmonics $(n>1)$ rapidly decrease in value as 
compared to the fundamental harmonic $(n=1)$, thus

$$
P \approx P_{D C}\left[c_{1, s} E_{0} \sin (\omega t)+c_{1, c} E_{0} \cos (\omega t)\right] .
$$

One can therefore fit the measured hysteresis loops with the fundamental harmonic only, to extract simply the values for $c_{1, s}$ and $c_{1, c}$ from which $\tan \delta_{P}$ can be calculated straightforwardly. The polarization hysteresis loops are obtained by high-pass filtering the signal and then averaging over the 20 measured cycles. The filtered data and the fits for a frequency of $3 \mathrm{kHz}$ are shown in $6.7 \mathrm{a}$ ) and b) and for other frequencies in Supporting Information B.4. The good fits of the polarization against time or field demonstrate that the contributions from the higher harmonics are not observable except for the high applied field values, thus that Eq. 6.13 gives an accurate approximation of the polarization hysteresis signal. Only for $E_{0} \geq 20 \mathrm{kV} \mathrm{cm}^{-1}$ one observes deviations from the fit with the fundamental harmonic. In these cases the magnitude of the second harmonic in the Fourier spectrum becomes comparable to that of the fundamental frequency.

a)

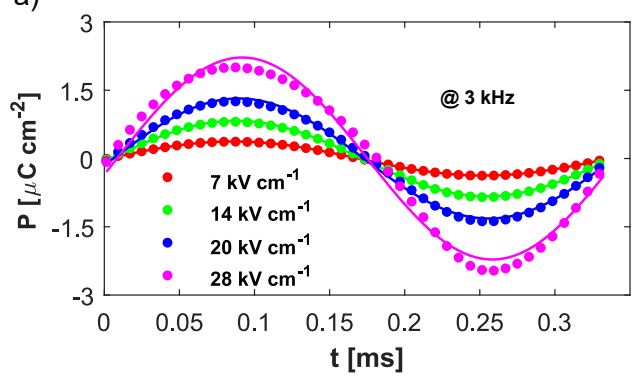

b)

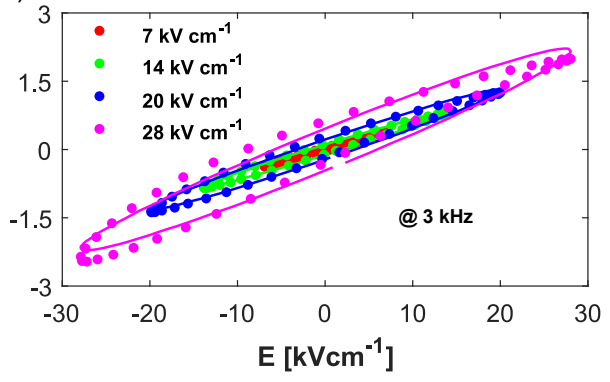

Figure 6.7: Filtered and averaged polarization hysteresis signal as function of a) time and b) applied AC electric field. The markers represent the measured data, which include all higher harmonics, and the solid lines are obtained by fitting to Eq. 6.13.

The polarization loss tangent, calculated with Eq. 6.12, is shown in Fig. 6.8 a) and $\mathrm{b}$ ) as a function of field amplitude and frequency, respectively. The loss tangent increases strongly with the increasing field amplitude. The polarization rotation model does not explicitly predict the observed field dependence but only a frequency dependence $\left|\omega \gamma_{P}\right|$ for a constant $\gamma_{P}$. There is a very weak explicit field dependence through the non-approximated version of the ratio $\left|c_{1, c} / c_{1, s}\right|$ which in fact would produce a opposite trend, i.e. a slight decrease of the loss tangent with increasing $E_{0}$. We neglect here this weak field dependence of the viscous loss term. Formally one can absorb the field dependence of the loss tangent in a field dependence of the viscosity parameter as in Lucke et.al. [24] However, it is not possible to include the non-zero offset in the frequency plots in this way. Moreover, since we did not find a field dependence for the strain loss tangent, we think that the field dependence is not part of the viscous loss, but can be attributed to an additional loss mechanism, which is only present in the polarization hysteresis and described by $\tan \delta_{E}=c E_{0}^{2}$. Although a linear description would fit equally well 
the bias case, we assume that the loss mechanism is the same as for the zero bias case, where a quadratic fit was required, therefore we use the same functional dependence on the field. [24] Comparing the loss tangent for the bias and zero bias cases in Fig. $6.8 \mathrm{a}$ ) shows that the loss tangent is drastically reduced when a bias field is applied. For example at $E_{0}=10 \mathrm{kV} \mathrm{cm}^{-1}$ the loss tangent in the case of bias is reduced by about a factor 3, which we attribute to the reduction of the prefactor $c$ of the field dependent term, which is thus a function of the bias point, $c\left(E_{D C}\right)$.

The frequency dependence of the loss tangent at different field amplitudes is shown in Fig. 6.8 b). The loss tangent increases linearly with frequency for frequencies above about $500 \mathrm{~Hz}$, while below this frequency the loss tangent increases slightly with decreasing frequency, suggesting a loss mechanism that may be related to $1 / f$-noise. We will not consider the latter any further here. The linear dependence above $500 \mathrm{~Hz}$ is typical for a viscous loss process and is predicted by the polarization rotation model in Eq. 6.12. Above we already showed that the amplitude dependence leads to a loss tangent contribution, $\tan \delta_{E}=c E_{0}^{2}$, while we also need to add a constant loss tangent, $\tan \delta_{0}$, to describe the offset of the viscous loss. For frequencies above about $500 \mathrm{~Hz}$ the total polarization loss tangent can now approximately be described with:

$$
\tan \delta_{P}\left(E_{0}, \omega\right) \approx\left|\gamma_{P} \omega\right|+c E_{0}^{2}+\tan \delta_{0}
$$

To extract the model parameters of Eq. 6.14, we have fitted the frequency dependence from $500 \mathrm{~Hz}$ to $5 \mathrm{kHz}$ with a linear function, $\tan \delta_{P}\left(E_{0}, \omega\right)=\tan \delta_{0}^{*}\left(E_{0}\right)+$ $\left|\gamma_{P} \omega\right|$, where $\tan \delta_{0}^{*}$ is a free fitting parameter depending on the field amplitude. A single $\gamma_{P}$ value is obtained from simultaneous fitting of all curves by least-square fitting. This procedure is allowed since the viscous contribution is assumed to be independent of $E_{0}$. The resulting linear fits are shown in Fig. $6.8 \mathrm{~b}$ ) by the lines and well describe the measured data. To extract $\tan \delta_{0}$ and $c$ the resulting $\tan \delta_{0}^{*}\left(E_{0}\right)$ values are fitted with $\tan \delta_{0}^{*}\left(E_{0}\right)=c E_{0}^{2}+\tan \delta_{0}$ as shown in Supporting Information B.5. For a bias of $20 \mathrm{kV} \mathrm{cm}{ }^{-1}$ the fit parameters are $\gamma_{P}=-3.27 \times 10^{-6} \operatorname{rad}^{-1} \mathrm{~Hz}^{-1}$, $c=1.46 \times 10^{-4} \mathrm{kV}^{-2} \mathrm{~cm}^{2}$ and $\tan \delta_{0}=4.34 \times 10^{-2}$. The same fitting procedure is repeated for the zero bias case, resulting in the fit parameters

$\gamma_{P}=-2.26 \times 10^{-6} \mathrm{rad}^{-1} \mathrm{~Hz}^{-1}, c=2.59 \times 10^{-3} \mathrm{kV}^{-2} \mathrm{~cm}^{2}$ and $\tan \delta_{0}=6.53 \times 10^{-2}$. The fits are shown in Supporting Information B.5. We observe that the application of a bias slightly changes $\gamma_{P}$, and $\tan \delta_{0}$, whereas the $c$-parameter is a factor 18 larger for the zero bias case. We attribute these differences to the much stronger curvature of the polarization loop at zero bias, and therefore stronger dependence of the polarization angle on the AC-field amplitude. Using these fit parameters and Eq. 6.14 the lines in Fig. 6.8 a) are generated and we observe that our model describes the measured loss tangent very well for the bias and zero bias case. These results strongly suggest that there are multiple loss mechanisms at play simultaneously in the polarization hysteresis: a viscous loss arising from the proposed polarization rotation model that accounts for the strong frequency dependence, a yet unexplained field dependent loss and an unexplained constant loss contribution. We note that the different loss mechanisms all appear to give rise to an elliptically shaped hysteresis loop, thus that they can be described by a relation like Eq. 6.13, 
i.e. with a $90^{\circ}$ out-of- phase response term and not by a Rayleigh-like description, that would give rise to more lenticular-shaped hysteresis loops.
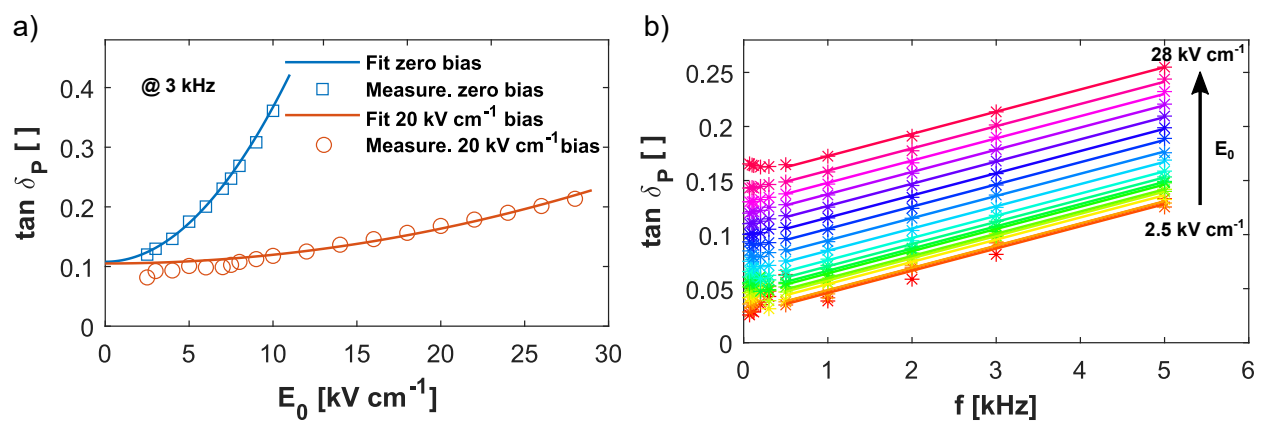

Figure 6.8: The polarization loss tangent as a function of a) field amplitude at $3 \mathrm{kHz}$ and b) frequency for varying field amplitudes.

Above we have applied the polarization rotation model, introduced in Lucke et. al., to describe the strain and polarization hysteresis in the same sample as discussed there, for the case of non-zero bias, by adding a DC-bias field term. [24] Furthermore we have used the model to a much wider applied field range, i.e. in the range from $-8 \mathrm{kV} \mathrm{cm}^{-1}$ to $48 \mathrm{kV} \mathrm{cm}^{-1}$. It is found that, although the strain response is hysteretic, the amplitude of the strain response has a linear relation with the applied field and no higher strain harmonics are observed. The strain response can therefore be described by a linear response model including a viscous loss proportional to the velocity of the strain change, $\Delta \dot{S}$. Moreover it is found that the parameters appearing in the hysteresis model, i.e. the effective piezoelectric coefficient $d_{33, f}$ and the strain loss parameter $\gamma_{S}$ do not depend on excitation frequency and amplitude in the investigated range. Consequently the loss tangent of the strain hysteresis process is simply given by the product of a constant $\gamma_{S}$ and the angular frequency, $\tan \delta_{S}=\left|\omega \gamma_{S}\right|$. Contrary to the strain the response of the polarization angle $\theta$ to the applied field is highly nonlinear. This causes many higher order harmonics in the polarization hysteresis signal. The model describes the polarization hysteresis with a viscous loss proportional to the velocity by which the polarization angle rotates $\Delta \dot{\theta}$, with a viscosity coefficient $\gamma_{P}$. However, experimentally an additional term quadratic in the field amplitude, but independent of frequency, and a constant loss term are found, which can not be explained by the polarization rotation model in its present form. We speculate that the amplitude dependent loss term is related to the oscillation of the amount of bound charges in charged domain walls due to the angular oscillation of the polarization vectors in the domains at both sides of the domain wall. This would show up in the dielectric properties of the film and thus in the polarization hysteresis, but not in the mechanical, hence strain properties. This would explain why for the strain loss tangent no field amplitude dependence is observed. For the constant loss term $\tan \delta_{0}$ we speculate that it could be a material dependent term, that describes the energy needed to initialize the polarization rotation. As such the decrease of this value for the bias case is reasonable, as the 
bias field has already moved the polarization vector out of its equilibrium position and further movement is easier. As the measurement noise for the strain response is much higher then for the polarization response, we are not sensible to the existence of such a term in the strain case. It is finally remarked that it is the extensive data set resulting from our study into the frequency and field amplitude dependence of the loss tangent that allows us to distinguish possibly different loss mechanisms in the same film. It also shows that several loss mechanisms with similar strength can be involved simultaneously. These results may be useful for further analysis with existing or new models for polarization loss.

\subsection{Conclusions}

In summary we observe hysteretic strain behaviour for an applied AC field superimposed on a DC bias field with a linear AC field amplitude response of the strain and a nonlinear, hysteretic polarization response in an epitaxial $\mathrm{PbZr}_{0.55} \mathrm{Ti}_{0.45} \mathrm{O}_{3}$ film with a monoclinic symmetry. The strain and polarization amplitude responses are well explained by an extension of the polarization rotation model, by taking into account the effect of a DC bias field on the rotation of the polarization vector. The polarization rotation model with applied bias, can predict the reduction of the nonlinearity of the polarization amplitude response for the case of non-zero field bias as compared to the zero bias case. The strain loss tangent is fully accounted for by the viscous term in the strain model. Of interest is the observed, additional field dependent term in the polarization loss tangent, which is attributed to a lossy charge motion mechanism in the ferroelectric film.

\section{Acknowledgments}

This work is part of the research programme "Smart Multilayer Interactive Optics for Lithography at Extreme UV wavelengths (SMILE)" with contract number 10448, and is financially supported by the Nederlandse Organisatie voor Wetenschappelijk Onderzoek (NWO) and Carl Zeiss SMT. This research has been carried out in the Industrial Focus Group XUV Optics and the Inorganic Material Science Group at the MESA + Institute for Nanotechnology of the University of Twente. The Industrial Focus Group XUV Optics receives further support from the Province of Overijssel, ASML and Malvern Panalytical. 


\section{References}

[1] R. Waser, Ceramic Materials for Electronics; Processing, Properties, and Applications, in Advanced Materials, Vol. 4 (Wiley, New York 1991, 1992) Book section 311, pp. 311-311, 2nd ed.

[2] N. Setter, D. Damjanovic, L. Eng, G. Fox, S. Gevorgian, S. Hong, A. Kingon, H. Kohlstedt, N. Y. Park, G. B. Stephenson, I. Stolitchnov, A. K. Taganstev, D. V. Taylor, T. Yamada, and S. Streiffer, Ferroelectric thin films: Review of materials, properties, and applications, Journal of Applied Physics 100, 051606 (2006).

[3] P. Muralt, Recent Progress in Materials Issues for Piezoelectric MEMS, Journal of the American Ceramic Society 91, 1385 (2008).

[4] P. Muralt, R. G. Polcawich, and S. Trolier-McKinstry, Piezoelectric Thin Films for Sensors, Actuators, and Energy Harvesting, MRS Bulletin 34, 658 (2009).

[5] C.-B. Eom and S. Trolier-McKinstry, Thin-film piezoelectric MEMS, MRS Bulletin 37, 1007 (2012).

[6] M. Bayraktar, W. A. Wessels, C. J. Lee, F. A. van Goor, G. Koster, G. Rijnders, and F. Bijkerk, Active multilayer mirrors for reflectance tuning at extreme ultraviolet (EUV) wavelengths, Journal of Physics D: Applied Physics 45, 494001 (2012).

[7] M. Bayraktar, A. Chopra, G. Rijnders, K. Boller, and F. Bijkerk, Wavefront correction in the extreme ultraviolet wavelength range using piezoelectric thin films, Optics Express 22, 30623 (2014).

[8] M. Nematollahi, P. Lucke, M. Bayraktar, A. Yakshin, G. Rijnders, and F. Bijkerk, Nanoscale piezoelectric surface modulation for adaptive extreme ultraviolet and soft x-ray optics, Optics Letters 44, 5104 (2019).

[9] H. Yang, F. Yan, Y. Lin, and T. Wang, Novel Strontium Titanate-Based Lead-Free Ceramics for High-Energy Storage Applications, ACS Sustainable Chemistry \& Engineering 5, 10215 (2017).

[10] J. O. Gentner, P. Gerthsen, N. A. Schmidt, and R. E. Send, Dielectric losses in ferroelectric ceramics produced by domain-wall motion, Journal of Applied Physics 49, 4485 (1978).

[11] B. Laikhtman, Flexural vibrations of domain walls and dielectric dispersion of ferroelectrics, Soviet Physics Solid State 15, 62 (1973).

[12] V. Postnikov, V. Pavlov, S. Gridnev, and S. Turkov, Interaction between 90 domain walls and point defects of the crystal lattice in ferroelectric ceramics, Physics of the Solid State 10, 1267 (1968). 
[13] V. Postnikov, V. Pavlov, S. Gridnev, B. Darinskii, and I. Glozman, Internal friction in $\mathrm{Pb}_{0.95} \mathrm{Sr}_{0.05} \mathrm{Zr}_{0.53} \mathrm{Ti}_{0.47} \mathrm{O}_{3}+3 \% \mathrm{PbO}$ ferroelectric ceramic, Bulletin of the Russian Academy of Sciences: Physics 31, 1888 (1967).

[14] G. Arlt and H. Dederichs, Complex elastic, dielectric and piezoelectric constants by domain wall damping in ferroelectric ceramics, Ferroelectrics 29, 47 (1980).

[15] D. Damjanovic and M. Demartin, The Rayleigh law in piezoelectric ceramics, Journal of Physics D: Applied Physics 29, 2057 (1996).

[16] L. Rayleigh, XXV. Notes on electricity and magnetism.-III. On the behaviour of iron and steel under the operation of feeble magnetic forces, The London, Edinburgh, and Dublin Philosophical Magazine and Journal of Science 23, 225 (1887).

[17] L. Néel, Théories des lois d'aimantation de Lord Rayleigh, Cahiers de physique 12, 1 (1942).

[18] L. Néel, Théorie des lois d'aimantation de Lord Rayleigh, 2, Cahiers de physique 13, 18 (1943).

[19] G. Bertotti, Energetic and Thermodynamic Aspects of Hysteresis, Phys. Rev. Lett. 76, 1739 (1996).

[20] G. Bertotti, V. Basso, and G. Durin, Random free energy model for the description of hysteresis, Journal of Applied Physics 79, 5764 (1996).

[21] K. Vergeer, Structure and functional properties of epitaxial $\mathrm{PbZr}_{x} \mathrm{Ti}_{1-x} \mathrm{O}_{3}$ films, Thesis, Universiteit Twente (2017).

[22] S. Trolier-McKinstry, N. Bassiri Gharb, and D. Damjanovic, Piezoelectric nonlinearity due to motion of $180^{\circ}$ domain walls in ferroelectric materials at subcoercive fields: A dynamic poling model, Applied Physics Letters 88, 202901 (2006).

[23] R. E. Eitel, T. R. Shrout, and C. A. Randall, Nonlinear contributions to the dielectric permittivity and converse piezoelectric coefficient in piezoelectric ceramics, Journal of Applied Physics 99, 124110 (2006).

[24] P. Lucke, M. Bayraktar, Y. A. Birkhölzer, M. Nematollahi, A. Yakshin, G. Rijnders, F. Bijkerk, and E. P. Houwman, Hysteresis, Loss and Nonlinearity in Epitaxial $\mathrm{PbZr}_{0.55} \mathrm{Ti}_{0.45} \mathrm{O}_{3}$ Films: A Polarization Rotation Model, Advanced Functional Materials, 2005397 (2020).

[25] N. Bassiri-Gharb, I. Fujii, E. Hong, S. Trolier-Mckinstry, D. V. Taylor, and D. Damjanovic, Domain wall contributions to the properties of piezoelectric thin films, Journal of Electroceramics 19, 47 (2007).

[26] D. Damjanovic and M. Demartin, Contribution of the irreversible displacement of domain walls to the piezoelectric effect in barium titanate and lead zirconate titanate ceramics, Journal of Physics: Condensed Matter 9, 4943 (1997). 
[27] D. V. Taylor, D. Damjanovic, E. Colla, and N. Setter, Fatigue and nonlinear dielectric response in sol-gel derived lead zirconate titanate thin films, Ferroelectrics 225, 91 (1999).

[28] B. Negulescu, C. J. M. Daumont, J. Sakai, A. Ruyter, M. Bavencoffe, N. Alyabyeva, and J. Wolfman, Nonlinear piezoelectric properties of epitaxial $\mathrm{BaTiO}_{3}$ thin film, Ferroelectrics 514, 9 (2017).

[29] E. P. Houwman, K. Vergeer, G. Koster, and G. Rijnders, Functional Properties of Polydomain Ferroelectric Oxide Thin Films, in Correlated Functional Oxides (Springer, 2017) Book section Chapter 2, pp. 29-53.

[30] E. Houwman, K. Vergeer, G. Koster, and G. Rijnders, Modelling functional properties of ferroelectric oxide thin films with a three-domain structure, arXiv preprint arXiv:1901.10883 (2019). 



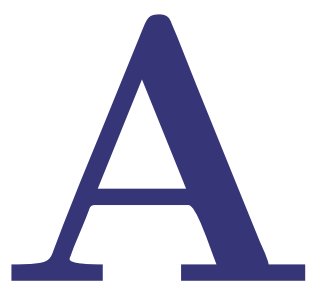

Supporting Information Hysteresis, loss and nonlinearity in epitaxial $\mathrm{PbZr}_{0.55} \mathrm{Ti}_{0.45} \mathrm{O}_{3}$ films: $\mathrm{A}$ polarization rotation model 


\section{A.1 Rayleigh model and its extensions}

In its basic form the Rayleigh model relates the material response $R$, i.e. for a ferroelectric material the piezoelectric strain $S$ or the dielectric polarization $P$, to the driving oscillating electric or stress field $F$ as:

$$
R(F)=\left(m_{\text {init }}+\alpha_{m} F_{0}\right) F \pm \frac{\alpha_{m}}{2}\left(F_{0}^{2}-F^{2}\right) .
$$

The driving signal $F$ can be an electric field or stress modulation and is usually taken as a sinusoidal field with amplitude $F_{0}$ with angular frequency $\omega=2 \pi f$, $F(t)=F_{0} \sin (\omega t)$. Here $m_{\text {init }}$ describes the field independent part of the piezoelectric coefficient $d$ or dielectric permittivity $\varepsilon$ due to intrinsic lattice response and the reversible DW contribution. $\alpha_{m}$ is the Rayleigh coefficient that describes the hysteresis, but also causes a nonlinear field response. The sign of the hysteretic term represents respectively the ascending $(-)$ and descending $(+)$ sections of the field cycle. The material parameter $m\left(F_{0}\right)$, corresponds to the observed dielectric permittivity $\varepsilon$ or piezoelectric coefficient $d$ :

$$
m\left(F_{0}\right)=m_{\text {init }}+\alpha_{m} F_{0}
$$

The Rayleigh model gives a phenomenological description of the hysteresis and nonlinearity of the strain and polarization as a function of the amplitude of an applied sinusoidal electric field. [1-4]

A characteristic for Rayleigh behavior is the lentil shape of the $S$ - $E$ and $P-E$ hysteresis loops, especially the sharp tips of the loop as shown in Fig. A.1a) for a hypothetical Rayleigh type polarization hysteresis loop. The (average) slope, indicated by the dotted line, is described by the first term in Eq. A.1 and represents the non-hysteretic linear part of the polarization, whereas the hysteresis, due to the non-linear response is reflected in the opening of the loop at zero field, described by the second term of Eq. A.1. The maximum opening is $\alpha_{m} F_{0}^{2}$ at zero field $F(t)=0$. Another characteristic of the Rayleigh model is that the Fourier expansion of Eq. A.1 only gives rise to odd order harmonics. This model has been used to describe the observed hysteresis for among others tetragonal, rhombohedral and morphotropic phase boundary (MPB) PZT ceramics and for early tetragonal CSD thin films. [1-10]

Robert et al. introduced viscous losses in the Rayleigh model to describe the polarization in response to stress in addition to Rayleigh-like losses. [1, 11]

$$
R(F)=\left[\left(m_{\text {init }}+\Delta m\right)+\alpha_{m} F_{0}\right] F \pm \frac{\alpha_{m}}{2}\left(F_{0}^{2}-F^{2}\right)+\gamma_{m} \Delta m \dot{F}
$$

Here $\gamma_{m}$ is the piezoelectric (or dielectric) viscosity coefficient, $\Delta m$ the piezoelectric (dielectric) amplitude of the viscous interaction and $\dot{F}$ the time derivative 
of the driving force. Note that the viscous term represents linear response with a lagging phase angle. As a result of the viscous loss term, the shape of the P-E loop changes from lentil shaped to a more elliptical shape: the tips of the loop become more rounded (see Fig. A.1b)). The higher the viscous losses the more elliptical the loop. However, still only odd order harmonics arise in the Fourier expansion of the response.

a)

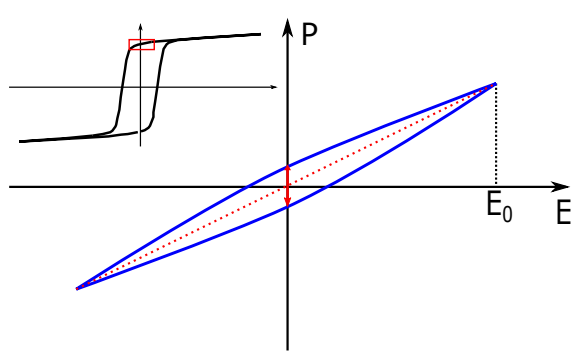

b)

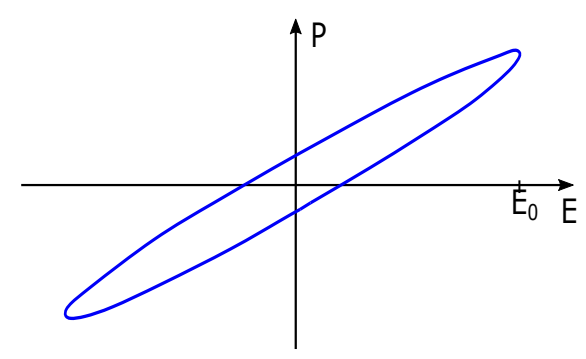

Figure A.1: a) Shape of the P-E hysteresis loop below the coercive field according to the Rayleigh model. The dotted red line shows the slope of the polarization. The small insets shows the global P-E loop for $\left|E_{\max }\right| \gg\left|E_{c}\right|$, in which the red box denotes the position of the sub-coercive field hysteresis loop with amplitude $E_{0}<\left|E_{c}\right|$. b) gives the shape of the sub-coercive field hysteresis $\mathrm{P}-\mathrm{E}$ loop taking into account a viscous loss mechanism.

Bassari Gharb et al. proposed a further variation of the Rayleigh model for the explanation of the observed strong second harmonic in the strain hysteresis in tetragonal, piezoelectric thin-films. [12-14] They added the possibility of $180^{\circ}$ domain wall motion (i.e. polarization reversal). The second harmonic (in addition to the usual odd harmonics) then arises from an AC-field and a field amplitude dependent reversible poling/depoling contribution to the piezoelectric coefficient. The strain electric field dependence is described by:

$$
S=\left(d_{0}\left[1+\left(\beta+\beta^{\prime} E_{0}\right) \sin (\omega t)\right]+\alpha_{d} E_{0}\right) E \pm \frac{\alpha_{d}}{2}\left(E_{0}^{2}-E^{2}\right)
$$

with $\beta$ and $\beta^{\prime}$ as scaling factors for the poling/depoling effect. Note that the polarization hysteresis is still given by the usual Rayleigh relation. We note that one would expect that a polarization reversal mechanism also shows up in the polarization hysteresis description and one would expect within this model therefore a second harmonic in the response, which however was not observed experimentally by the authors.

The energy loss (per unit volume) per hysteresis cycle is equal to the area of the hysteresis loop, which in the Rayleigh model of Eq. A.1 is given by: [1]

$$
A_{R}=\int_{\text {cycle }} R(E) d E=\frac{4 \alpha_{m} E_{0}^{3}}{3}
$$


Adding a viscous term to the Rayleigh model (Eq. A.3) gives an additional linear viscous response $R_{\mathrm{visc}}=R_{0} \sin (\omega t-\delta)$ on the driving field $E=E_{0} \sin (\omega t)$ and the associated additional loop area is

$$
A_{\mathrm{visc}}=\int_{\text {cycle }} R_{\mathrm{visc}}(E) d E=\pi R_{0} E_{0} \sin (\delta)=-\pi \gamma_{m} \Delta m \omega E_{0}^{2} .
$$

The loss tangent is in general defined as the tangent of the angle between the fundamental harmonic of the signal $R$ and the driving field $E$ and can thus analogous to the above relation be written as

$$
\tan \delta \approx \delta \approx \sin (\delta)=\frac{A_{\mathrm{loss}}}{\pi R_{0} E_{0}}
$$

where $A_{\text {loss }}$ is the area of the hysteresis loop. Applying this formalism to the Rayleigh model of Eq. A.1, one finds:

$$
\tan \delta_{R}=\frac{A_{R}}{\pi R_{0} E_{0}}=\frac{4 \alpha_{m} E_{0}}{3 \pi\left(m_{\mathrm{init}}+\alpha_{m} E_{0}\right)} .
$$

The additional loss angle due to viscous interaction in the Rayleigh model is

$$
\delta_{\mathrm{visc}} \approx \tan \delta_{\mathrm{visc}}=-\frac{\gamma_{m} \Delta m \omega}{\left(m_{\mathrm{init}}+\Delta m+\alpha_{m} E_{0}\right)}
$$

\section{A.2 Strain hysteresis derived from Landau-Devonshire theory}

From the Landau-Devonshire (LD) description of a monodomain, ferroelectric film clamped to a substrate one obtains the following expression for the strain in the zdirection $S_{3}$ (index 1, 2, 3 correspond to in-plane x- and y-direction and out-of-plane z-direction respectively). [15]

$$
S_{3}=a+b\left(P_{1}^{2}+P_{2}^{2}\right)+c P_{3}^{2}
$$

Here $a=\frac{2 s_{12}}{s_{11}+s_{12}} S_{m}^{0}, b=Q_{12}-\frac{2 s_{12}}{s_{11}+s_{12}}\left(Q_{11}+Q_{12}\right), c=Q_{11}-\frac{2 s_{12}}{s_{11}+s_{12}} Q_{12}$, with $s_{i j}$ the elastic coefficients, $Q_{i j}$ the electrostrictive coupling coefficients, $S_{m}^{0}$ is the substrate-induced strain and $P_{i}$ are the polarization components. For thick films the substrate-induced strain is mainly due to the difference in thermal expansion coefficients between film and substrate, since at deposition temperature the bulk of the film can be assumed to be fully relaxed from epitaxial strain due to the incorporation of (dislocation) defects. [16, 17]

For the description of a monoclinic film also 8th order terms are needed to describe the monoclinic symmetry without applied field. [18] However, as the coefficients of these terms are very small, the energy of these terms should be negligible small 
compared to the energy of the terms coming from the strain.

In a rhombohedral symmetry that under influence of strain trsforms into monoclinic symmetry and monoclinic unit cell symmetries, the polarization rotates in the (110)-plane and one can write $P_{3}=P_{0} \cos (\theta)$ and $P_{1}=P_{2}=P_{0} \sin (\theta) / \sqrt{2}$, with $\theta$ the angle between the polarization vector with length $P_{0}$ and the z-axis. With this one can rewrite the strain as

$$
\begin{aligned}
S_{3} & =\left(a+b P_{0}^{2}\right)+P_{0}^{2}(c-b) \cos ^{2}(\theta) \\
& =\left[a+\frac{P_{0}^{2}}{2}(c+b)\right]+\frac{P_{0}^{2}}{2}(c-b) \cos (2 \theta)=A+B \cos (2 \theta) .
\end{aligned}
$$

Here we have defined $A=a+\frac{P_{0}^{2}}{2}(c+b)$ and $B=\frac{P_{0}^{2}}{2}(c-b)$. We assume that under an applied field in the 3 -direction the polarization extension (lengthening of $P_{0}$ vector) is small and that the change in the measurable polarization in the 3 direction is predominantly due to polarization rotation ${ }^{1}$.

In the main manuscript it was stated that the field dependence of the rotation angle can be parameterized as

$$
\theta(E)=\theta_{0}+\Delta \theta(E)+\gamma_{P} \Delta \dot{\theta}(E) \approx \theta_{0}+\beta_{1} E+\gamma_{P} \beta_{1} \dot{E}+\beta_{2} E^{2}+\beta_{3} E^{3}
$$

Here we neglected higher order terms involving $\dot{E}$.

Expanding $\cos (2 \theta)=\cos \left(2 \theta_{0}\right) \cos \left(2\left(\Delta \theta+\gamma_{P} \Delta \dot{\theta}\right)\right)-\sin \left(2 \theta_{0}\right) \sin \left(2\left(\Delta \theta+\gamma_{P} \Delta \dot{\theta}\right)\right) \approx$ $\cos \left(2 \theta_{0}\right)-2\left(\Delta \theta+\gamma_{P} \Delta \theta\right) \sin \left(2 \theta_{0}\right)$, and substituting $\Delta \theta(E), \Delta \dot{\theta}(E)$ one finds for $S_{3}$ up to second order in $E$ and first order in $\dot{E}$ :

$$
S_{3} \approx A+B \cos \left(2 \theta_{0}\right)-2 B \sin \left(2 \theta_{0}\right)\left[\beta_{1} E+\gamma_{P} \beta_{1} \dot{E}+\beta_{2} E^{2}+\beta_{3} E^{3}\right] .
$$

Thus, the measured strain change is,

$$
\begin{aligned}
\Delta S_{3}(E) & =S_{3}(E)-S_{3,0}=-2 B \sin \left(2 \theta_{0}\right)\left[\beta_{1} E+\gamma_{P} \beta_{1} \dot{E}+\beta_{2} E^{2}+\beta_{3} E^{3}\right] \\
S_{3,0} & =A+B \cos \left(2 \theta_{0}\right)
\end{aligned}
$$

Eq. A.15 relates the zero-field angle $\theta_{0}$ to the zero-field strain. For small amplitude oscillatory fields, i.e $\left|\beta_{2} E_{0}+\beta_{3} E_{0}^{2}\right| /\left|\beta_{1}\right| \ll 1$, which is true for $E_{0}<$ $10 \mathrm{kV} \mathrm{cm}^{-1}$ within $10 \%$ accuracy, one finds a linear response.

$$
\Delta S_{3}(t) \approx\left[2 B \sin \left(2 \theta_{0}\right) E_{0}\right]\left[-\beta_{1} \sin (\omega t)-\omega \gamma_{P} \beta_{1} \cos (\omega t)\right]
$$

\footnotetext{
${ }^{1}$ In ref. [16, 17] it was shown, by Landau-Devonshire modelling of a clamped rhombohedral film on STO with PZT 60/40 composition, that the contribution to polarization change due to polarization rotation is a factor 3.2 larger than for polarization extension.
} 
Comparing this expression with Eq. 5.2 in the main manuscript we can identify the hysteresis parameters with the expressions obtained from LD-modelling as:

$$
\begin{aligned}
d_{33, f}(L D) & =-2 B \sin \left(2 \theta_{0}\right) \beta_{1} \\
\gamma_{S}(L D) & =\gamma_{P}
\end{aligned}
$$

Thus although in the main manuscript we assumed different loss parameters for strain and polarization loss, here it is shown that they are the same. With hindsight this might have been expected because of the linear coupling between polarization and strain in the linearized model of piezoelectrics, $S=d_{33, f} E$, where $d_{33, f}=2 Q_{\text {eff }} \varepsilon_{0} \varepsilon_{\text {eff }} P$. Using (interpolated) literature values for $\operatorname{PZT}(55 / 45)$, $Q_{11}=0.0846 \mathrm{~m}^{4} \mathrm{C}^{-2}, Q_{12}=-0.0365 \mathrm{~m}^{4} \mathrm{C}^{-2}, s_{11}=9.65 \times 10^{-12} \mathrm{~m}^{2} \mathrm{~N}^{-1}$, $s_{12}=-3.30 \times 10^{-12} \mathrm{~m}^{2} \mathrm{~N}^{-1}, P_{0}=0.5 \mathrm{C} \mathrm{m}^{-2}$ one finds $d_{33, f}(L D)=312 \mathrm{pm} \mathrm{V}^{-1}$. [15, 19] This is of the same order of magnitude as the experimentally determined values $d_{33, f}(\exp )=96 \mathrm{pm} \mathrm{V}^{-1}$. The difference is attributed to the fact that the above derivation is based on a LD-model for a single domain, clamped, single crystal epitaxial film, whereas in the experiment we are dealing with a polydomain, polyepitaxial, clamped film, in which the strain response is changed by the grain boundaries and domain walls. We consider the numerical correspondence between experimental and calculated hysteresis parameters to be satisfactory and conclude that the LDdescription of the strain allows for a connection between the strain and polarization response.

\section{A.3 Amplitude of the harmonics of the polarization hysteresis}

In the main manuscript we have shown that the time-dependent polarization response is given by Eq. 5.7. The time independent bias term of Eq. 5.7 is:

$$
\begin{aligned}
c_{0}= & -\left(\frac{9 \beta_{3}^{2} \gamma_{P}^{2} \omega^{2}}{32}+\frac{5 \beta_{3}^{2}}{32}\right) E_{0}^{6}-\left(\frac{\beta_{2}^{2} \gamma_{P}^{2} \omega^{2}}{4}+\frac{3 \beta_{2}^{2}}{16}+\frac{3 \beta_{1} \beta_{3} \gamma_{P}^{2} \omega^{2}}{8}+\frac{3 \beta_{1} \beta_{3}}{8}\right) E_{0}^{4} \\
& -\left(\frac{\beta_{1}^{2} \gamma_{P}^{2} \omega^{2}}{4}+\frac{\beta_{1}^{2}}{4}+\frac{\beta_{2} \tan \left(\theta_{0, f}\right)}{2}\right) E_{0}^{2}+1 \approx 1
\end{aligned}
$$

The last approximation holds within less than $10^{-3}$ for all experimental field amplitudes and frequencies using the values for the fit parameters given in Fig. 5.1d) and the value for $\gamma_{P}$ extracted at the end of Section 5.4.4 of the main manuscript. The coefficients of the time dependent harmonics are:

$$
\begin{aligned}
c_{1, s}= & -\left(\frac{3 \beta_{2} \beta_{3} \gamma_{P}^{2} \omega^{2}}{4}+\frac{5 \beta_{2} \beta_{3}}{8}\right) E_{0}^{4}-\left(\frac{\beta_{1} \beta_{2} \gamma_{P}^{2} \omega^{2}}{2}+\frac{3 \beta_{1} \beta_{2}}{4}+\frac{3 \beta_{3} \tan \left(\theta_{0, f}\right)}{4}\right) E_{0}^{2} \\
& -\beta_{1} \tan \left(\theta_{0, f}\right) \\
\approx & -\beta_{1}\left[\left(\frac{3 \beta_{2}}{4}+\frac{3 \beta_{3} \tan \left(\theta_{0, f}\right)}{4 \beta_{1}}\right) E_{0}^{2}+\tan \left(\theta_{0, f}\right)\right]
\end{aligned}
$$




$$
\begin{aligned}
& c_{1, c}=-\frac{5 \beta_{2} \beta_{3} \gamma_{P} \omega}{8} E_{0}^{4}-\left(\frac{\beta_{3} \gamma_{P} \omega \tan \left(\theta_{0, f}\right)}{4}+\frac{3 \beta_{1} \beta_{2} \gamma_{P} \omega}{4}\right) E_{0}^{2}-\beta_{1} \gamma_{P} \omega \tan \left(\theta_{0, f}\right) \\
& \approx-\beta_{1} \gamma_{P} \omega\left[\left(\frac{3 \beta_{3} \tan \left(\theta_{0, f}\right)}{4 \beta_{1}}+\frac{3 \beta_{2}}{4}\right) E_{0}^{2}+\tan \left(\theta_{0, f}\right)\right] \\
& c_{2, s}=-\frac{15 \beta_{3}^{2} \gamma_{P} \omega}{32} E_{0}^{4}-\left(\frac{\beta_{2}^{2} \gamma_{P} \omega}{2}+\beta_{1} \beta_{3} \gamma_{P} \omega\right) E_{0}^{2}-\left(\frac{\beta_{1}^{2} \gamma_{P} \omega}{2}+\beta_{2} \gamma_{P} \omega \tan \left(\theta_{0}\right)\right) \\
& \approx-\gamma_{P} \omega\left[\left(\frac{\beta_{2}^{2}}{2}+\beta_{1} \beta_{3}\right) E_{0}^{2}+\left(\frac{\beta_{1}^{2}}{2}+\beta_{2} \tan \left(\theta_{0}\right)\right)\right] \\
& c_{2, c}=\left(\frac{9 \beta_{3}^{2} \gamma_{P}^{2} \omega^{2}}{64}+\frac{15 \beta_{3}^{2}}{64}\right) E_{0}^{4}+\left(\frac{\beta_{2}^{2}}{4}+\frac{\beta_{1} \beta_{3}}{2}\right) E_{0}^{2}+\left(-\frac{\beta_{1}^{2} \gamma_{P}^{2} \omega^{2}}{4}+\frac{\beta_{1}^{2}}{4}+\frac{\beta_{2} \tan \left(\theta_{0}\right)}{2}\right) \\
& \approx\left(\frac{\beta_{2}^{2}}{4}+\frac{\beta_{1} \beta_{3}}{2}\right) E_{0}^{2}+\left(-\frac{\beta_{1}^{2} \gamma_{P}^{2} \omega^{2}}{4}+\frac{\beta_{1}^{2}}{4}+\frac{\beta_{2} \tan \left(\theta_{0}\right)}{2}\right) \\
& c_{3, s}=\left(\frac{5 \beta_{2} \beta_{3}}{16}-\frac{3 \beta_{2} \beta_{3} \gamma_{P}^{2} \omega^{2}}{8}\right) E_{0}^{2}+\left(-\frac{\beta_{1} \beta_{2} \gamma_{P}^{2} \omega^{2}}{2}+\frac{\beta_{1} \beta_{2}}{4}+\frac{\beta_{3} \tan \left(\theta_{0}\right)}{4}\right) \\
& c_{3, c}=\frac{15 \beta_{2} \beta_{3} \gamma_{P} \omega}{16} E_{0}^{2}+\left(\frac{3 \beta_{3} \gamma_{P} \omega \tan \left(\theta_{0}\right)}{4}+\frac{3 \beta_{1} \beta_{2} \gamma_{P} \omega}{4}\right) \\
& c_{4, s}=\frac{3 \beta_{3}^{2} \gamma_{P} \omega}{8} E_{0}^{2}+\left(\frac{\beta_{2}^{2} \gamma_{P} \omega}{4}+\frac{\beta_{1} \beta_{3} \gamma_{P} \omega}{2}\right) \\
& c_{4, c}=\left(\frac{9 \beta_{3}^{2} \gamma_{P}^{2} \omega^{2}}{32}-\frac{3 \beta_{3}^{2}}{32}\right) E_{0}^{2}+\left(\frac{\beta_{2}^{2} \gamma_{P}^{2} \omega^{2}}{4}-\frac{\beta_{2}^{2}}{16}+\frac{3 \beta_{1} \beta_{3} \gamma_{P}^{2} \omega^{2}}{8}-\frac{\beta_{1} \beta_{3}}{8}\right) \\
& c_{5, s}=\left(\frac{3 \beta_{2} \beta_{3} \gamma_{P}^{2} \omega^{2}}{8}-\frac{\beta_{2} \beta_{3}}{16}\right) \\
& c_{5, c}=-\frac{5 \beta_{2} \beta_{3} \gamma_{P} \omega}{16} \\
& c_{6, s}=-\frac{3 \beta_{3}^{2} \gamma_{P} \omega}{32} \\
& c_{6, c}=\left(\frac{\beta_{3}^{2}}{64}-\frac{9 \beta_{3}^{2} \gamma_{P}^{2} \omega^{2}}{64}\right)
\end{aligned}
$$


Table A.1: The amplitudes of different harmonics.

\begin{tabular}{cccccc}
\hline $\begin{array}{c}\text { Harmonic } \\
\boldsymbol{n}\end{array}$ & $\mathbf{c}_{\mathbf{n}, \mathbf{s}}$ & $\mathbf{c}_{\mathbf{n}, \mathbf{c}}$ & Unit & Character & $\mathbf{P}_{\mathbf{n}} / \mathbf{P}_{\mathbf{r}, \mathbf{f}}$ \\
\hline 0 & 1 & 1 & & & \\
1 & $3.6 \times 10^{-3}$ & $-1.3 \times 10^{-3}$ & $\left(\mathrm{kV} \mathrm{cm}^{-1}\right)^{-1}$ & Offset & 1 \\
2 & $3.7 \times 10^{-5}$ & $4.9 \times 10^{-5}$ & $\left(\mathrm{kV} \mathrm{cm}^{-1}\right)^{-2}$ & $\mathrm{CW}$ & $3.9 \times 10^{-2}$ \\
3 & $-1.6 \times 10^{-6}$ & $1.8 \times 10^{-6}$ & $\left(\mathrm{kV} \mathrm{cm}^{-1}\right)^{-3}$ & $\mathrm{CCW}$ & $2.3 \times 10^{-3}$ \\
4 & $-7.0 \times 10^{-9}$ & $-2.7 \times 10^{-9}$ & $\left(\mathrm{kV} \mathrm{cm}^{-1}\right)^{-4}$ & $\mathrm{CW}$ & $7.5 \times 10^{-5}$ \\
5 & $8.8 \times 10^{-12}$ & $-9.2 \times 10^{-11}$ & $\left(\mathrm{kV} \mathrm{cm}^{-1}\right)^{-5}$ & $\mathrm{CCW}$ & $9.2 \times 10^{-6}$ \\
6 & $1.8 \times 10^{-12}$ & $-1.9 \times 10^{-12}$ & $\left(\mathrm{kV} \mathrm{cm}^{-1}\right)^{-6}$ & $\mathrm{CCW}$ & $1.8 \times 10^{-6}$ \\
\hline
\end{tabular}

The (relative) amplitudes $P_{n} / P_{r, f}=k_{n, m} E_{0}^{n}=E_{0}^{n} \sqrt{c_{n, s}^{2}+c_{n, c}^{2}}$ of the different harmonics of the polarization hysteresis were calculated from the fitted values of $\theta(E)($ Fig. $5.1 \mathrm{~d}))$ for $E_{0}=10 \mathrm{kV} \mathrm{cm}^{-1}$ and $f=3 \mathrm{kHz}$ and $\gamma_{P}\left(E_{0}=10 \mathrm{kV} \mathrm{cm}^{-1}\right)=$ $-2 \times 10^{-5} \mathrm{~Hz}^{-1} \mathrm{rad}^{-1}$.

The rotation direction of the hysteresis, described with Lissajous-type functions $(\mathrm{i}, \mathrm{i})$, is counter clockwise $(\mathrm{CCW})$ if $c_{n, s} c_{n, c}<0$, and clockwise $(\mathrm{CW})$ if this product is positive. It is seen that the amplitude of the first order harmonics is about $4 \%$ of the remanent polarization value, and for higher order harmonics the amplitude rapidly decreases, according to the model.

For a $3 \mathrm{kHz}, \quad 10 \mathrm{kV} \mathrm{cm}^{-1}$ excitation field the measured ratio $P_{1, \text { meas. }} / P_{r, f} \approx 1.35 / 38.9=3.5 \times 10^{-2}$ which is very close to the calculated value of $3.9 \times 10^{-2}$ (the polarization response is shown in Fig. $5.2 \mathrm{e}$ ), and the $P_{r, f}$ is extracted from the full polarization loop, Fig. 5.1c). Fig. A.2 shows the calculated amplitudes, normalized to the amplitude of the first order harmonic $P_{n} / P_{r, f}$. Also shown are the measured amplitudes (determined as the amplitude of the peaks in the Fourier spectrum of Fig. 5.2f), normalized to the measured amplitude of the first order harmonic, $P_{n \text {,meas. }} / P_{r, f}$. Further the determined $1 / f$-noise level is indicated.

It is observed that the measured amplitudes of the $2^{\text {nd }}$ and $3^{\text {rd }}$ harmonic are smaller than the calculated values, whereas those of the $4^{\text {th }}-6^{\text {th }}$ harmonic are significantly higher. The amplitudes of the $4^{t h}$ and $5^{t h}$ harmonic have become so large compared to the calculated values that they rise above the $1 / f$-noise level. We speculate that energy of the lower harmonics is transferred to higher harmonics by a non-linear process that is not taken into account in the model of the hysteresis. This may also be the reason for the complicated structure of the polarization Fourier spectrum in Fig. 5.2f) that shows significant broadening of the $2^{\text {nd }}$ and $3^{\text {rd }}$ harmonic and suppression of the $1 / f$-noise spectrum around these frequencies.

In the main manuscript we have given general expressions for the AC-field am- 


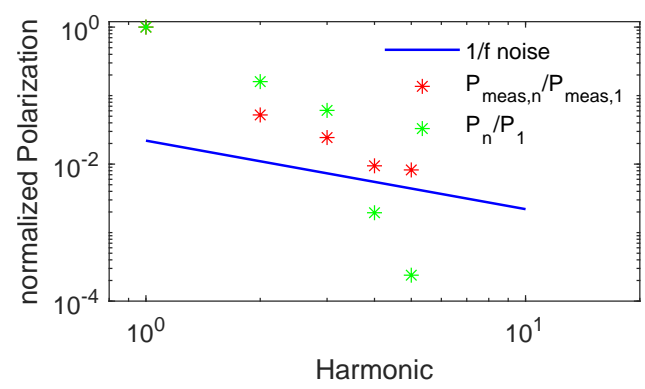

Figure A.2: Amplitude of $n^{\text {th }}$ harmonic normalized to the amplitude of the $1^{\text {st }}$ harmonic, as calculated from the model (green markers) and as measured (red markers). The blue line indicates the measured $1 / f$ polarization noise.

plitude dependence of the harmonics in terms of a polynomial expansion in $E_{0}$, with general polynomial coefficients $k_{n, m}$ (Eq. 5.8). Below we give the full expressions.

$$
\begin{aligned}
\left|P_{1}\right| / P_{r, f} & =E_{0} \sqrt{\left(c_{1, s}^{2}+c_{1, c}^{2}\right)} \\
& =E_{0}\left\{\left[\left(\frac{3 \beta_{2} \beta_{3} \gamma_{P}^{2} \omega^{2}}{4}+\frac{5 \beta_{2} \beta_{3}}{8}\right) E_{0}^{4}-\left(\frac{\beta_{1} \beta_{2} \gamma_{P}^{2} \omega^{2}}{2}+\frac{3 \beta_{1} \beta_{2}}{4}+\frac{3 \beta_{3} \tan \left(\theta_{0, f}\right)}{4}\right) E_{0}^{2}\right.\right. \\
& \left.-\beta_{1} \tan \left(\theta_{0, f}\right)\right]^{2}+\left[\frac{5 \beta_{2} \beta_{3} \gamma_{P} \omega}{8} E_{0}^{4}-\left(\frac{\beta_{3} \gamma_{P} \omega \tan \left(\theta_{0, f}\right)}{4}+\frac{3 \beta_{1} \beta_{2} \gamma_{P} \omega}{4}\right) E_{0}^{2}\right. \\
& \left.\left.-\beta_{1} \gamma_{P} \omega \tan \left(\theta_{0, f}\right)\right]^{2}\right\}^{1 / 2} \\
& \approx E_{0} \beta_{1} \tan \left(\theta_{0, f}\right) \sqrt{1+\left(\gamma_{P} \omega\right)^{2}} \\
& +E_{0}^{3} \frac{\left(\frac{\beta_{1} \beta_{2} \gamma_{P}^{2} \omega^{2}}{2}+\frac{3 \beta_{1} \beta_{2}}{4}+\frac{3 \beta_{3} \tan \left(\theta_{0, f}\right)}{4}\right)+\left(\frac{\beta_{3} \gamma_{P}^{2} \omega^{2} \tan \left(\theta_{0, f}\right)}{4}+\frac{3 \beta_{1} \beta_{2} \gamma_{P}^{2} \omega^{2}}{4}\right)}{\sqrt{1+\left(\gamma_{P} \omega\right)^{2}}} \\
& =k_{1,1} E_{0}+k_{1,3} E_{0}^{3}
\end{aligned}
$$

Thus $k_{1,1} \approx \beta_{1} \tan \left(\theta_{0, f}\right)$ if $\left(\gamma_{P} \omega\right)^{2} \ll 1$ and $k_{1,3} \approx \frac{3 \beta_{1} \beta_{2}}{4}$ when $\frac{\beta_{3}}{\beta_{1} \beta_{2}} \ll 1$ and $\left(\gamma_{P} \omega\right)^{2} \ll 1$. 


$$
\begin{aligned}
\left|P_{2}\right| / P_{r, f} & =E_{0}^{2} \sqrt{\left(c_{2, s}^{2}+c_{2, c}^{2}\right)} \\
& =E_{0}^{2}\left\{\left[-\frac{15 \beta_{3}^{2} \gamma_{P} \omega}{32} E_{0}^{4}-\left(\frac{\beta_{2}^{2} \gamma_{P} \omega}{2}+\beta_{1} \beta_{3} \gamma_{P} \omega\right) E_{0}^{2}-\left(\frac{\beta_{1}^{2} \gamma_{P} \omega}{2}+\beta_{2} \gamma_{P} \omega \tan \left(\theta_{0}\right)\right)\right]^{2}\right. \\
& +\left[\left(\frac{9 \beta_{3}^{2} \gamma_{P}^{2} \omega^{2}}{64}+\frac{15 \beta_{3}^{2}}{64}\right) E_{0}^{4}+\left(\frac{\beta_{2}^{2}}{4}+\frac{\beta_{1} \beta_{3}}{2}\right) E_{0}^{2}\right. \\
& \left.\left.+\left(-\frac{\beta_{1}^{2} \gamma_{P}^{2} \omega^{2}}{4}+\frac{\beta_{1}^{2}}{4}+\frac{\beta_{2} \tan \left(\theta_{0}\right)}{2}\right)\right]^{2}\right\}^{1 / 2} \\
& \approx E_{0}^{2} \sqrt{\left(\frac{\beta_{1}^{2} \gamma_{P} \omega}{2}+\beta_{2} \gamma_{P} \omega \tan \left(\theta_{0}\right)\right)^{2}+\left(-\frac{\beta_{1}^{2} \gamma_{P}^{2} \omega^{2}}{4}+\frac{\beta_{1}^{2}}{4}+\frac{\beta_{2} \tan \left(\theta_{0}\right)}{2}\right)^{2}} \\
& +E_{0}^{4}\left(\frac{3 \beta_{1}^{2} \beta_{2}^{2} \gamma_{P}^{2} \omega^{2}}{16}+\frac{7 \beta_{1}^{3} \beta_{3} \gamma_{P}^{2} \omega^{2}}{8}+\beta_{2}^{3}\left(\frac{1}{8}+\frac{\gamma_{P}^{2} \omega^{2}}{2}\right)\right. \\
& +\frac{\sqrt{\left(\frac{\beta_{1}^{2} \gamma_{P} \omega}{2}+\beta_{2} \gamma_{P} \omega \tan \left(\theta_{0}\right)\right)^{2}+\left(-\frac{\beta_{1}^{2} \gamma_{P}^{2} \omega^{2}}{4}+\frac{\beta_{1}^{2}}{4}+\frac{\beta_{2} \tan \left(\theta_{0}\right)}{2}\right)^{2}}}{\left.\sqrt{\left(\frac{\beta_{1}^{2} \gamma_{P} \omega}{2}+\beta_{2} \gamma_{P} \omega \tan \left(\theta_{0}\right)\right)^{2}+\left(-\frac{\beta_{1}^{2} \gamma_{P}^{2} \omega^{2}}{4}+\frac{\beta_{1}^{2}}{4}+\frac{\beta_{2} \tan \left(\theta_{0}\right)}{2}\right)^{2}}\right)^{2}} \\
& =k_{2,2} E_{0}^{2}+k_{2,4} E_{0}^{4}
\end{aligned}
$$

$$
\begin{aligned}
\left|P_{3}\right| / P_{r, f} & =E_{0}^{3} \sqrt{\left(c_{3, s}^{2}+c_{3, c}^{2}\right)} \\
& =E_{0}^{3}\left\{\left[\left(\frac{5 \beta_{2} \beta_{3}}{16}-\frac{3 \beta_{2} \beta_{3} \gamma_{P}^{2} \omega^{2}}{8}\right) E_{0}^{2}+\left(-\frac{\beta_{1} \beta_{2} \gamma_{P}^{2} \omega^{2}}{2}+\frac{\beta_{1} \beta_{2}}{4}+\frac{\beta_{3} \tan \left(\theta_{0}\right)}{4}\right)\right]^{2}\right. \\
& \left.+\left[\frac{15 \beta_{2} \beta_{3} \gamma_{P} \omega}{16} E_{0}^{2}+\left(\frac{3 \beta_{3} \gamma_{P} \omega \tan \left(\theta_{0}\right)}{4}+\frac{3 \beta_{1} \beta_{2} \gamma_{P} \omega}{4}\right)\right]^{2}\right\}^{1 / 2} \\
& \approx E_{0}^{3} \sqrt{\left(-\frac{\beta_{1} \beta_{2} \gamma_{P}^{2} \omega^{2}}{2}+\frac{\beta_{1} \beta_{2}}{4}+\frac{\beta_{3} \tan \left(\theta_{0}\right)}{4}\right)^{2}+\left(\frac{3 \beta_{3} \gamma_{P} \omega \tan \left(\theta_{0}\right)}{4}+\frac{3 \beta_{1} \beta_{2} \gamma_{P} \omega}{4}\right)^{2}} \\
& +E_{0}^{5} \frac{\frac{5 \beta_{1} \beta_{2}^{2} \beta_{3}}{64}+\beta_{2} \beta_{3} \tan \left(\theta_{0}\right)\left(\frac{45 \gamma_{P} \omega}{64}-\frac{1}{64}\right)+\frac{29 \beta_{1} \beta_{2}^{2} \beta_{3} \gamma_{P}^{2} \omega^{2}}{64}+\frac{3 \beta_{1} \beta_{2}^{2} \gamma_{P}^{4} \omega^{4}}{16}}{\sqrt{\left(-\frac{\beta_{1} \beta_{2} \gamma_{P}^{2} \omega^{2}}{2}+\frac{\beta_{1} \beta_{2}}{4}+\frac{\beta_{3} \tan \left(\theta_{0}\right)}{4}\right)^{2}+\left(\frac{3 \beta_{3} \gamma_{P} \omega \tan \left(\theta_{0}\right)}{4}+\frac{3 \beta_{1} \beta_{2} \gamma_{P} \omega}{4}\right)^{2}}} \\
& =k_{3,3} E_{0}^{3}+k_{3,5} E_{0}^{5}
\end{aligned}
$$




$$
\begin{aligned}
& \left|P_{4}\right| / P_{r, f}=E_{0}^{4} \sqrt{\left(c_{4, s}^{2}+c_{4, c}^{2}\right)} \\
& =E_{0}^{4}\left\{\left[\frac{3 \beta_{3}^{2} \gamma_{P} \omega}{8} E_{0}^{2}+\left(\frac{\beta_{2}^{2} \gamma_{P} \omega}{4}+\frac{\beta_{1} \beta_{3} \gamma_{P} \omega}{2}\right)\right]^{2}\right. \\
& \left.+\left[\left(\frac{9 \beta_{3}^{2} \gamma_{P}^{2} \omega^{2}}{32}-\frac{3 \beta_{3}^{2}}{32}\right) E_{0}^{2}+\left(\frac{\beta_{2}^{2} \gamma_{P}^{2} \omega^{2}}{4}-\frac{\beta_{2}^{2}}{16}+\frac{3 \beta_{1} \beta_{3} \gamma_{P}^{2} \omega^{2}}{8}-\frac{\beta_{1} \beta_{3}}{8}\right)\right]^{2}\right\}^{1 / 2} \\
& \approx E_{0}^{4} \sqrt{\left(\frac{\beta_{2}^{2} \gamma_{P} \omega}{4}+\frac{\beta_{1} \beta_{3} \gamma_{P} \omega}{2}\right)^{2}+\left(\frac{\beta_{2}^{2} \gamma_{P}^{2} \omega^{2}}{4}-\frac{\beta_{2}^{2}}{16}+\frac{3 \beta_{1} \beta_{3} \gamma_{P}^{2} \omega^{2}}{8}-\frac{\beta_{1} \beta_{3}}{8}\right)^{2}} \\
& +E_{0}^{6}\left(\frac{\beta_{3}^{2} \gamma_{P}^{2} \omega^{2}\left(\frac{27}{512} \beta_{2}^{2}+\frac{15}{128} \beta_{1} \beta_{3}\right)+\beta_{3}^{2} \gamma_{P}^{4} \omega^{4}\left(\frac{9}{128} \beta_{2}^{2}+\frac{27}{256} \beta_{1} \beta_{3}\right)}{\sqrt{\left(\frac{\beta_{2}^{2} \gamma_{P} \omega}{4}+\frac{\beta_{1} \beta_{3} \gamma_{P} \omega}{2}\right)^{2}+\left(\frac{\beta_{2}^{2} \gamma_{P}^{2} \omega^{2}}{4}-\frac{\beta_{2}^{2}}{16}+\frac{3 \beta_{1} \beta_{3} \gamma_{P}^{2} \omega^{2}}{8}-\frac{\beta_{1} \beta_{3}}{8}\right)^{2}}}\right. \\
& \left.+\frac{\frac{3}{256} \beta_{2}^{2}\left(\beta_{1}+\frac{1}{2} \beta_{3}^{2}\right)}{\sqrt{\left(\frac{\beta_{2}^{2} \gamma_{P} \omega}{4}+\frac{\beta_{1} \beta_{3} \gamma_{P} \omega}{2}\right)^{2}+\left(\frac{\beta_{2}^{2} \gamma_{P}^{2} \omega^{2}}{4}-\frac{\beta_{2}^{2}}{16}+\frac{3 \beta_{1} \beta_{3} \gamma_{P}^{2} \omega^{2}}{8}-\frac{\beta_{1} \beta_{3}}{8}\right)^{2}}}\right) \\
& =k_{4,4} E_{0}^{4}+k_{4,6} E_{0}^{6} \\
& \left|P_{5}\right| / P_{r, f}=E_{0}^{5}\left\{\left[\left(\frac{3 \beta_{2} \beta_{3} \gamma_{P}^{2} \omega^{2}}{8}-\frac{\beta_{2} \beta_{3}}{16}\right)\right]^{2}+\left[-\frac{5 \beta_{2} \beta_{3} \gamma_{P} \omega}{16}\right]^{2}\right\}^{1 / 2}=k_{5,5} E_{0}^{5} \\
& \left|P_{6}\right| / P_{r, f}=E_{0}^{6}\left\{\left[-\frac{3 \beta_{3}^{2} \gamma_{P} \omega}{32}\right]^{2}+\left[\left(\frac{\beta_{3}^{2}}{64}-\frac{9 \beta_{3}^{2} \gamma_{P}^{2} \omega^{2}}{64}\right)\right]^{2}\right\}^{1 / 2}=k_{6,6} E_{0}^{6}
\end{aligned}
$$

\section{A.4 XRD analysis}

Fig. A.3 summarizes the results of the X-ray diffraction (XRD) measurements. Fig. A.3a) displays a conventional $\theta / 2 \theta$ scan, showing only peaks belonging to the 001 family of planes and no signs of impurity phases. We use pseudocubic Miller indices for the PZT and LNO to emphasize the epitaxial relation between the thin layers and the cubic STO substrate. Based on the bulk phase diagram of PZT, for the chosen slightly Zr-rich composition, one would anticipate a rhombohedral unit cell. However, when grown as a heteroepitaxial film on a substrate, the symmetry can be lowered by thermal and misfit strain. For PZT on LNO bottom electrodes, which grow coherently strained on STO, one may expect a unit cell which is elongated in the out-of-plane direction due to in-plane compressive strain from the STO substrate. Besides compression and elongation, misfit may also be accommodated by altering the angles of the individual unit cell, as well as through the formation of an intricate multi-domain structure with of a variety of tilting and twisting patterns of the constituent domains. Unfortunately, an exact refinement of the atomic coordinates is notoriously difficult for multi-domain thin films and beyond the scope of 
this study.

From $\theta / 2 \theta$ scans, only the out-of-plane lattice parameter can be determined. To determine the in-plane lattice parameters, several reciprocal space maps were measured. Fig. A.3b) shows the (103) out-of-plane (HL) reciprocal space map (RSM), which confirms that all layers are grown epitaxially with respect to the substrate. The LNO bottom electrode is in-plane coherently strained to the STO substrate, whereas the bulk of the PZT layer is largely relaxed, as the in-plane momentum $\left(q_{x}\right)$ differs significantly from that of the STO substrate. Two peaks with slightly different in-plane but equal out-of-plane momenta are observed in this projection. The reason for the peculiar horizontal splitting becomes clear by examining the in-plane (HK) RSM of the same feature shown in Fig. A.3c). The two peaks seen in the (HL) map actually each consist of two superimposed peaks in the k-direction $\left(q_{y}\right)$. These four observed reflections correspond directly to four distinct but rotationally invariant domains of unit cells with the long in-plane axis pointing towards the four $<110>$ directions.

Based on these measurements, the PZT unit cell is deduced to have equal inplane lattice parameters $a_{u c}=b_{u c}$, separated by an angle $\gamma_{u c}$. The $a$ - and $b$ - axes are tilted by a small angle $\chi_{1}$ from the substrate plane. The $c$-axis forms equal angles $\alpha_{u c}=\beta_{u c}$ with the $b$ - and $a$ - axis respectively, and is clearly parallel to the substrate normal, thus $\alpha_{u c}=\beta_{u c} \approx 90^{\circ}-\chi_{1}$.

In summary, the inferred lattice parameters are $a_{u c}=b_{u c}=4.081 \AA, c_{u c}=$ $4.097 \AA, \gamma_{u c} \approx 89.79^{\circ}, \alpha_{u c}=\beta_{u c} \approx 89.74^{\circ}$, and $\chi_{1}=0.26^{\circ}$. The unit cell volume $V_{u c} \approx a_{u c}^{2} c_{u c}=68.27 \AA^{3}$ and the pseudocubic lattice parameter $a_{p c}=V_{p c}^{1 / 3}=$ $4.087 \AA$. From these results we conclude that the pseudocubic unit cell is an oblique rhombic prism, belonging to the monoclinic crystal system, with a (110) symmetry plane, in line with our expectation. This interpretation is further supported by RSMs of the (004) and (113) features (not shown).

Pictorially, the STO surface lattice consists of a grid of perfect squares. On top, one may visualize the in-plane projection of the PZT lattice by a rhombus that is formed by pulling along the $\langle 110\rangle$ direction of the squares. Subsequently, the rhombohedron is tilted such that the far corner is lifted above the surface plane. Fig. A.4 depicts this structure schematically from the top (panel a) and the side (panel b).

This structure is different from more frequently reported MA or MC-type monoclinic lattices where the c-axis is tilted away from the substrate normal. Such a tilt would reveal itself in the out-of-plane RSMs by showing 2 or 3 peaks with different out-of-plane momenta $\left(q_{z}\right)$ in the (103) and (113) maps, for example. This is evidently not the case here.

All XRD data shown here has been measured on a pristine, i.e. uncycled and not deliberately poled, device with lateral dimensions of $300 \times 300 \mu^{2}$. 
a)

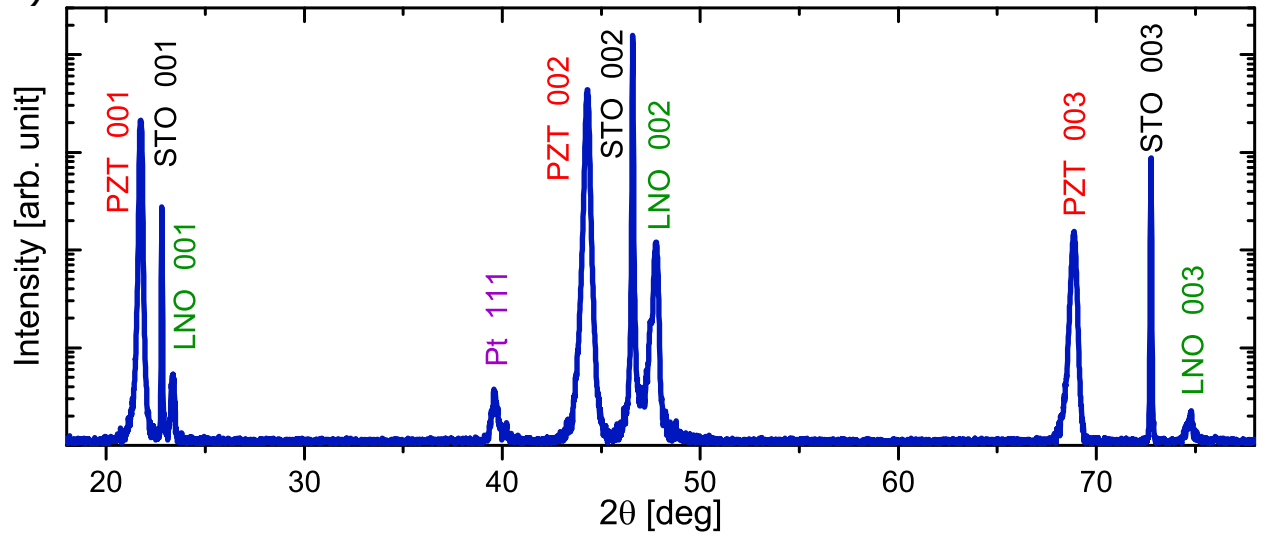

b)

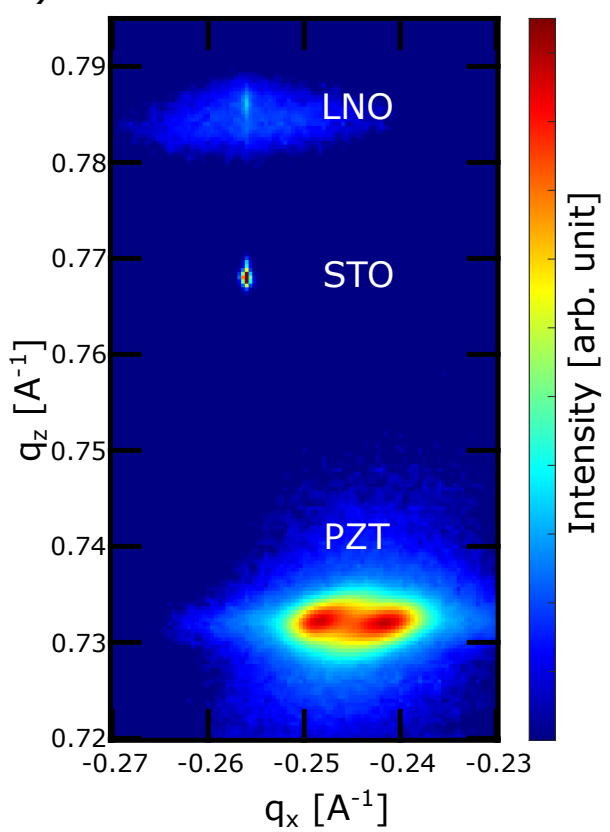

c)

C) $\quad 0.01$

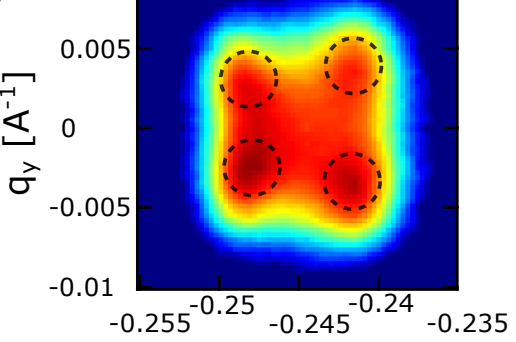

d)

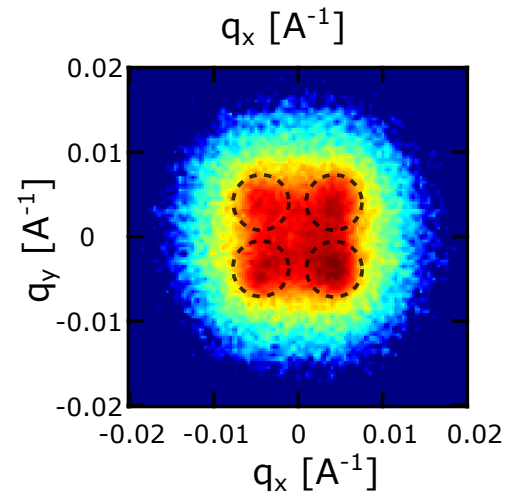

Figure A.3: a) $\theta / 2 \theta$-scan: no impurity phase is detected and only 001 peaks of the PZT are observed; b) hl-RSM around the -103 reflections indicating the epitaxial growth of the sample and the equal out-of-plane lattice parameter of all PZT domains; c) hk in-plane RSM around the -103 reflections of the four PZT domains, indicated by the four dashed circles; d) hk-RSM around 004 reflections, showing four nearly equally intense peaks. 
a)

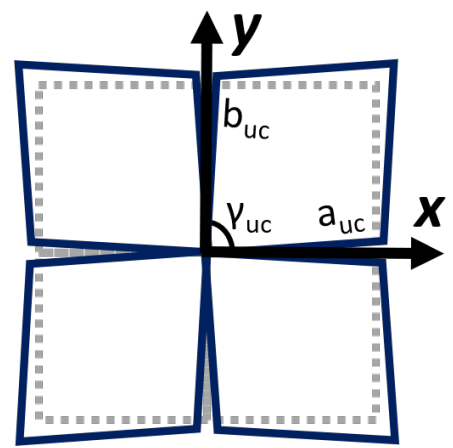

b)

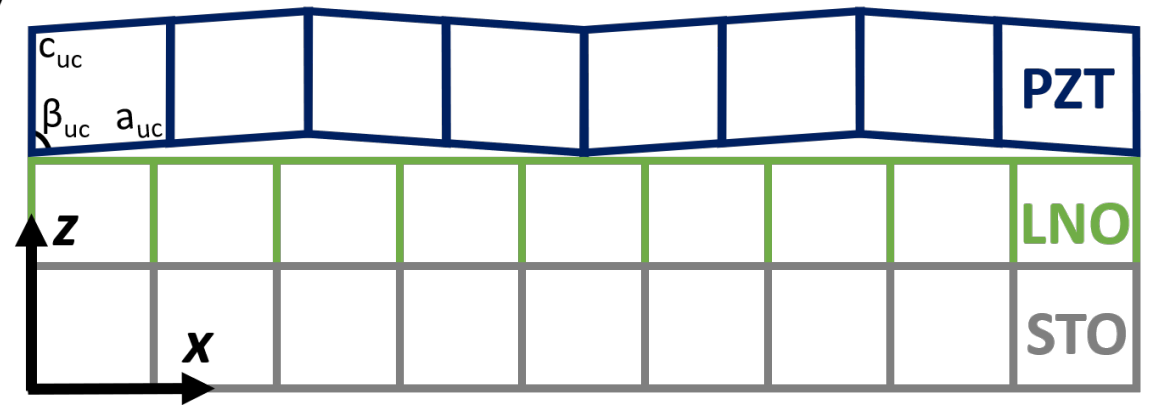

Figure A.4: Schematic drawing of the PZT, LNO, and STO lattices a) as seen from the top along the [001] direction and b) from the side along [010]. Dimensions and angles not to scale. 


\section{A.5 Small signal $d_{33}$ measurement}

Fig. A.5 displays the measured small signal piezoelectric coefficient plotted against the applied DC bias field at $3 \mathrm{kHz}$. The small signal was of trapezoidal shape with an amplitude of $1.7 \mathrm{kV} \mathrm{cm}^{-1}$. The measured loop shape is rectangular-like, as is expected for a good crystalline film. The extracted $d_{33, f}$ value $\left(93 \mathrm{pm} \mathrm{V} \mathrm{V}^{-1}\right)$ measured with a large signal in the main manuscript is in very good agreement with the $d_{33, f, s s}$ value $\left(96 \mathrm{pm} \mathrm{V}^{-1}\right)$ at $0 \mathrm{kV} \mathrm{cm}^{-1} \mathrm{DC}$-bias from Fig. A.5.

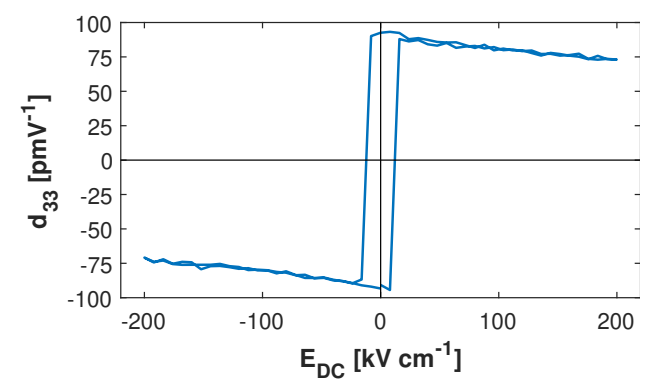

Figure A.5: Small signal $\left(1.7 \mathrm{kV} \mathrm{cm}^{-1}\right) d_{33, s s}$ versus applied DC-bias field scanning from -200 to $200 \mathrm{kV} \mathrm{cm}^{-1}$ range at $3 \mathrm{kHz}$.

\section{A.6 Rate dependence of fit parameters}

Tab. A.2 summarizes the changes of the remanent polarization and the coercive field with scanning field frequency $f_{s}$ and amplitude $E_{s}$. The large electric field $P-E$ loops have been measured from $5 \mathrm{kHz}$ down to $100 \mathrm{~Hz}$ with an $E_{0}$ of 200, 100 and $66.7 \mathrm{kV} \mathrm{cm}^{-1}$ respectively. Lower electric fields are not possible, as the loop become not saturated. One observes that the remanent polarization only changes minimal with frequency or applied field. The coercive field however, shows a strong dependence on both the applied field and the frequency. Both observations constitute violations of the assumptions underlying the polarization rotation model, namely that $\theta_{0, f}$ and all $\beta$ 's are assumed to be independent of the applied field and frequency, which has as consequence that both the position of the coercive field and the remanent polarization are independent of the applied field and frequency. The remanent polarization is directly related to $\theta_{0, f}$ and the position of the coercive field has an influence how non-linear the change of polarization with applied field is 
Table A.2: Change of remnant polarization and coercive field with frequency and amplitude.

\begin{tabular}{|c|c|c|c|c|c|}
\hline $\begin{array}{c}\mathbf{f}_{\mathbf{s}} \\
{[\mathbf{H z}]}\end{array}$ & $\begin{array}{c}\mathrm{E}_{\mathrm{s}} \\
{\left[\mathrm{kV} \mathrm{cm}^{-1}\right]}\end{array}$ & $\begin{array}{c}\mathbf{P}_{\mathbf{r}+} \\
{\left[\mu \mathbf{C ~ c m}^{-\mathbf{2}}\right]}\end{array}$ & $\begin{array}{c}\mathbf{P}_{\mathbf{r}-} \\
{\left[\mu \mathbf{C ~ c m}^{-\mathbf{2}}\right]}\end{array}$ & $\begin{array}{c}\mathbf{E}_{\mathbf{c}+} \\
{\left[\mathrm{kV} \mathrm{cm}^{-1}\right]}\end{array}$ & $\left.\begin{array}{c}\mathbf{E}_{\mathbf{c}-} \\
{\left[\mathrm{kV} \mathbf{c m}^{-1}\right.}\end{array}\right]$ \\
\hline 5000 & 200 & 40.3 & -37 & 39.9 & -32 \\
\hline 5000 & 67 & 39.2 & -37.9 & 24 & -22 \\
\hline 3000 & 200 & 38.5 & -40.9 & 31.2 & -29.6 \\
\hline 3000 & 100 & 38.7 & -38.4 & 24.6 & -22.3 \\
\hline 3000 & 67 & 38.3 & -38.5 & 21.8 & -19.4 \\
\hline 1000 & 200 & 39 & -38.9 & 24 & -21.2 \\
\hline 1000 & 100 & 38.6 & -38.6 & 20.1 & -17 \\
\hline 1000 & 67 & 38.3 & -38.3 & 18.4 & -16 \\
\hline 500 & 200 & 39 & -39.3 & 21.6 & -18.8 \\
\hline 500 & 100 & 38.5 & -38.7 & 18.7 & -15.8 \\
\hline 500 & 67 & 38 & -38.3 & 17.5 & -14.2 \\
\hline 100 & 200 & 39.3 & -39.5 & 18.3 & -15.3 \\
\hline 100 & 100 & 38.8 & -38.8 & 16.5 & -13.7 \\
\hline 100 & 67 & 38.5 & -38.5 & 15.6 & -13 \\
\hline
\end{tabular}

\section{A.7 Strain hysteresis loops at different frequencies}

Fig. A.6 and Fig. A.7 display the filtered and averaged strain signals measured at all measured frequencies respectively with respect to Fig. A.6 time and Fig. A.7 electric field at 3 selected amplitudes. As in the main manuscript in Fig. 5.3 only every fifth datapoint is shown with markers for clarity. The slopes of the $S-E$ loops in Fig. A.7 are proportional to $d_{33, f}$ and the loop-opening to the viscosity parameter. As can be seen, the slopes for different amplitudes are the same within the errorbars as explained in the main text and the loop openings are very small. The model fits that are shown with solid lines in Fig. A.6 and Fig. A.7 are perfectly matching the experimental data. All other measured amplitudes show similar behavior.

\section{A.8 Polarization hysteresis loops at different fre- quencies}

Fig. A.8 and Fig. A.9 show the polarization hysteresis with respect to Fig. A.8 time and Fig. A.9 electric field, at selected amplitudes for all measured frequencies. The data points, plotted as markers, were obtained by bandpass filtering at all harmonic frequencies and then averaged over the measured 20 cycles. The standard deviation of the average is less than the marker size. All higher harmonics are included in the loops. One clearly observes the elliptical shape of the hysteresis loops at all frequencies and amplitudes. The experimental loops were fitted with the fundamental harmonic only, depicted by the continuous lines in the figure, using Eq. 5.9, as described in the main manuscript, Section 5.4.4. The fits in time and field domains show that the contribution of the higher harmonics are only noticeable 
a)

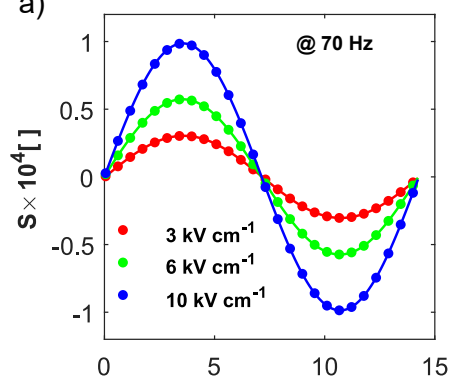

d)

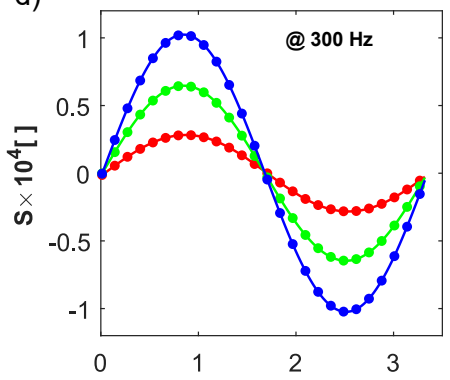

g)

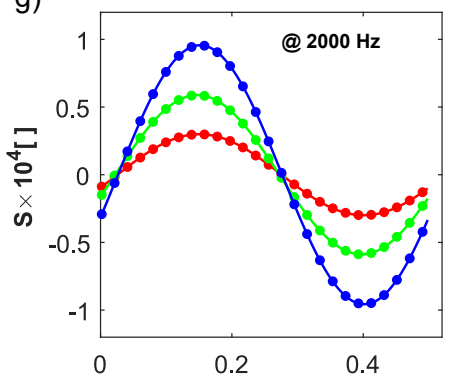

b)

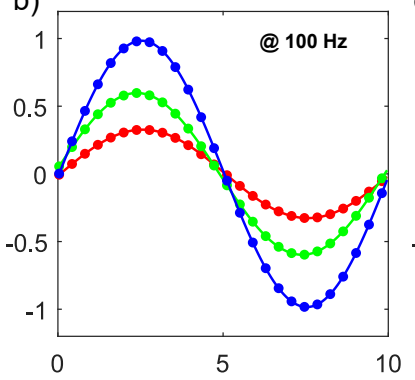

e)

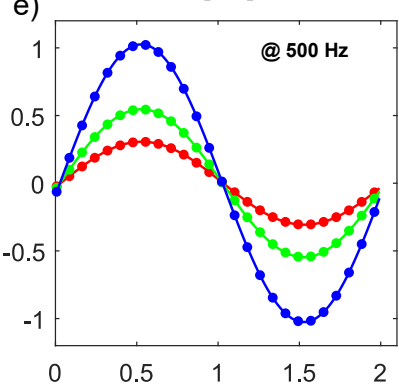

h) $\mathbf{t}[\mathrm{ms}]$

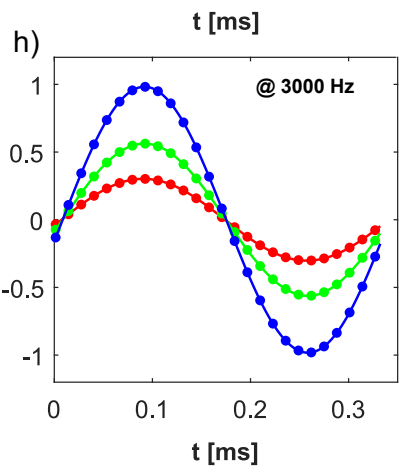

c)

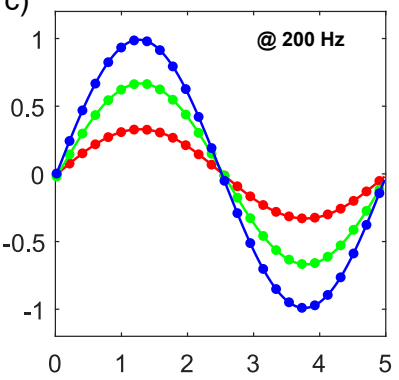

f)

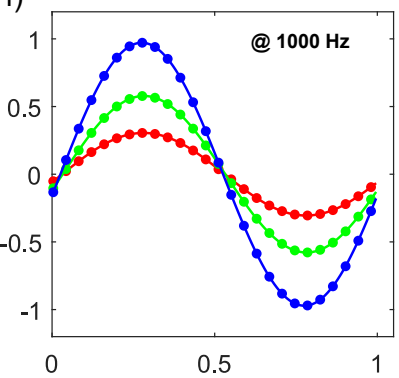

i)

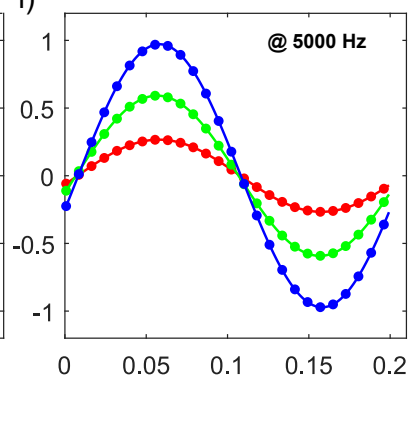

Figure A.6: Filtered and averaged strain signal plotted with respect to time, for three different field amplitudes at a) $70 \mathrm{~Hz}$, b) $100 \mathrm{~Hz}$, c) $200 \mathrm{~Hz}$, d) $300 \mathrm{~Hz}$, e) $500 \mathrm{~Hz}$, f) $1000 \mathrm{~Hz}$, g) $2000 \mathrm{~Hz}, \mathrm{~h}$ ) $300 \mathrm{~Hz}$ and i) $5000 \mathrm{~Hz}$. Datapoints show the measurements and the lines are obtained from fitting with Eq. 5.2.

at the peaks of the highest electric field amplitude. 
a)

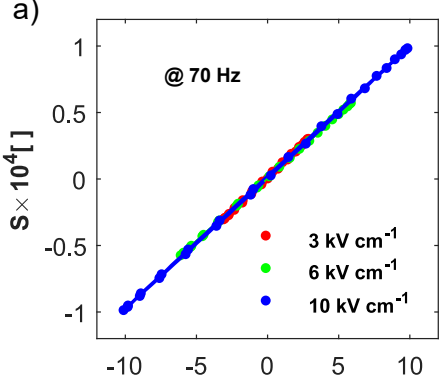

d)

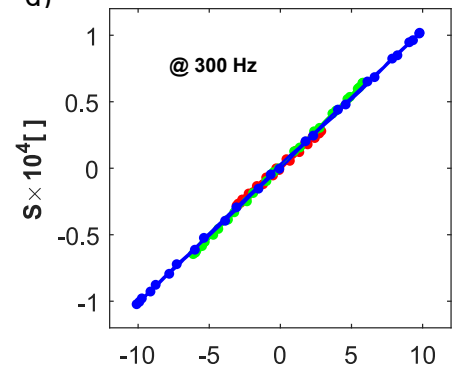

g)

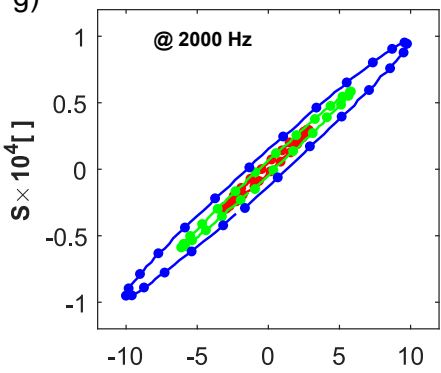

b)

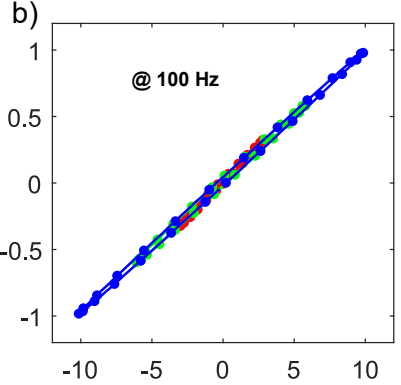

$E\left[\mathrm{kV} \mathrm{cm}{ }^{-1}\right]$

e)

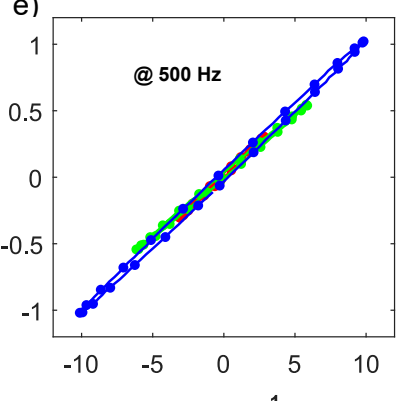

h)

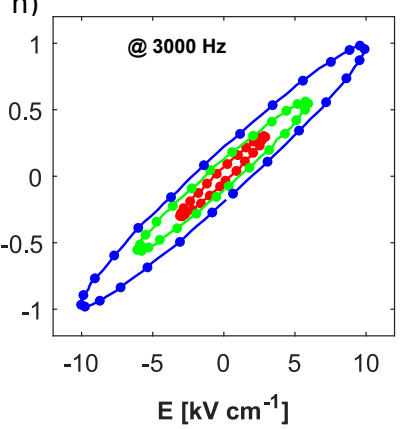

c)

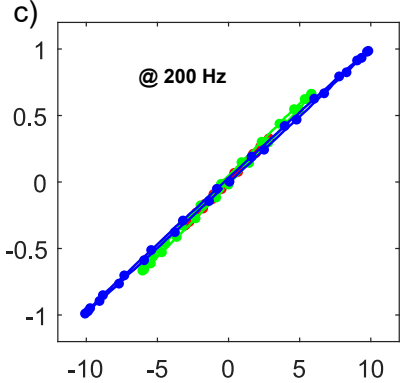

f)

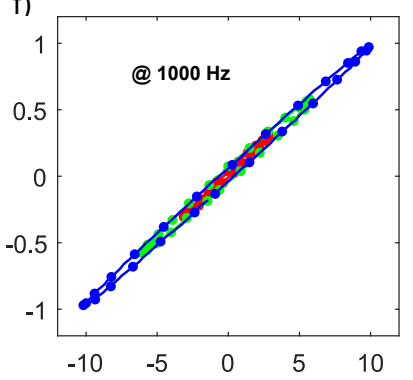

i)

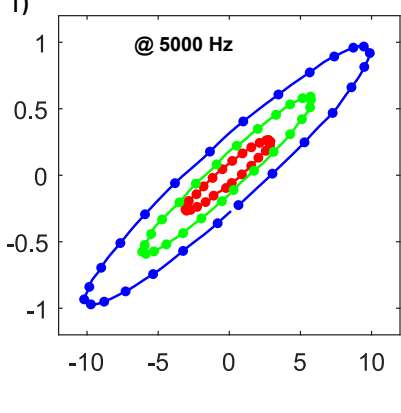

Figure A.7: Filtered and averaged strain signal plotted with respect to field, for three different field amplitudes at a) $70 \mathrm{~Hz}$, b) $100 \mathrm{~Hz}$, c) $200 \mathrm{~Hz}$, d) $300 \mathrm{~Hz}$, e) $500 \mathrm{~Hz}$, f) $1000 \mathrm{~Hz}$, g) $2000 \mathrm{~Hz}, \mathrm{~h}$ ) $300 \mathrm{~Hz}$ and i) $5000 \mathrm{~Hz}$. Datapoints show the measurements and the lines are obtained from fitting with Eq. 5.2. 
a)

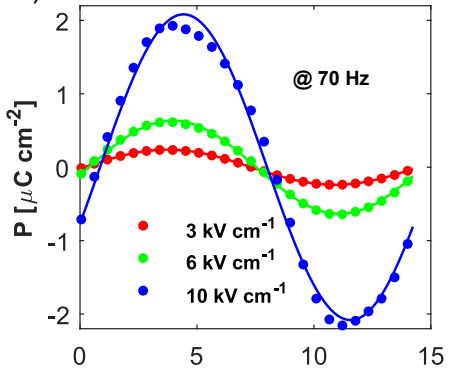

d)

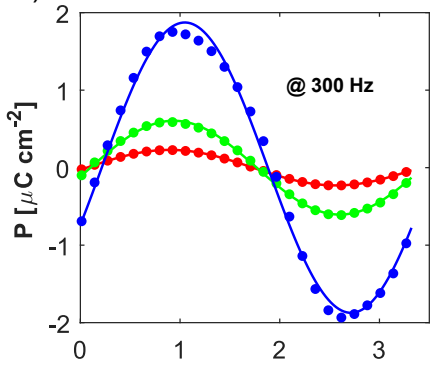

b)

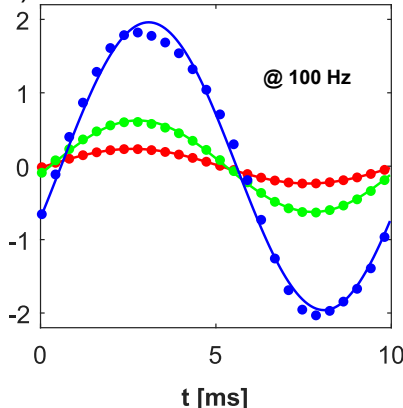

c)

e)
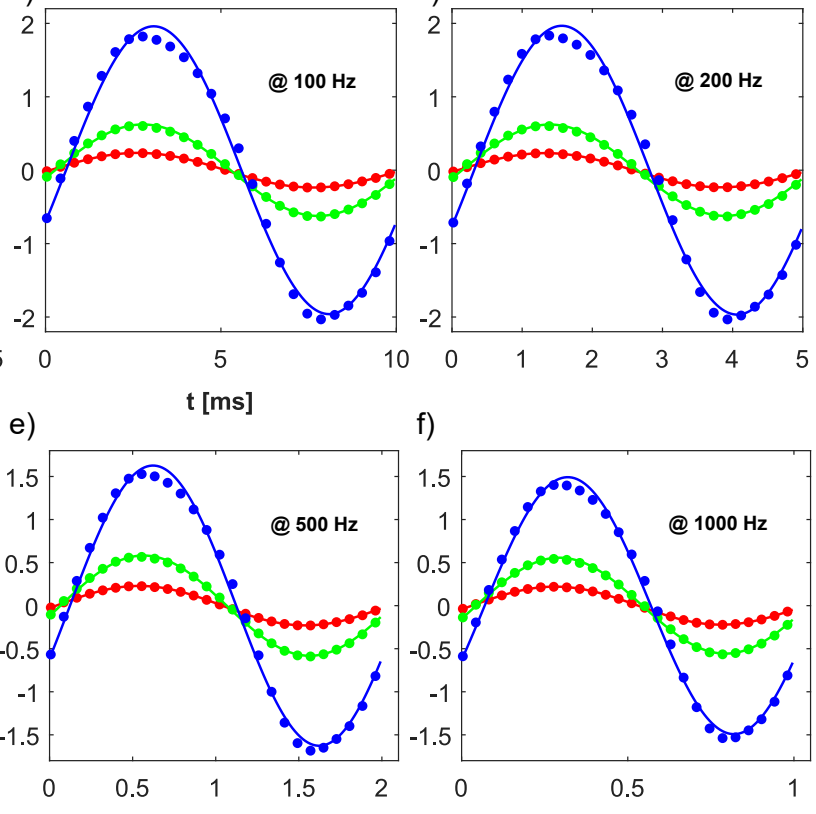

f)

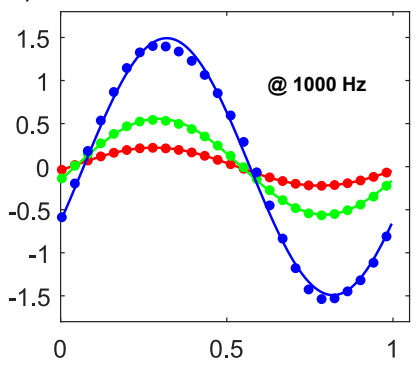

g)

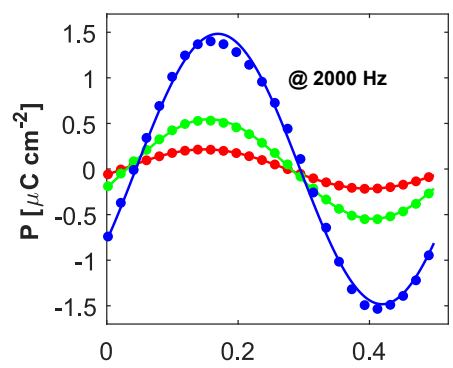

i)

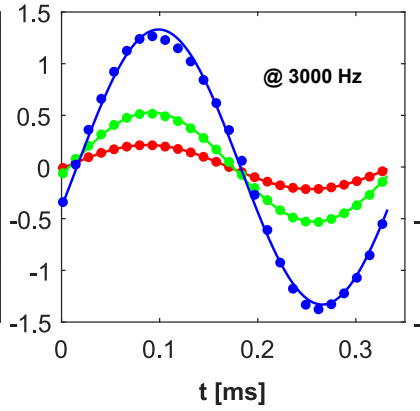

h)

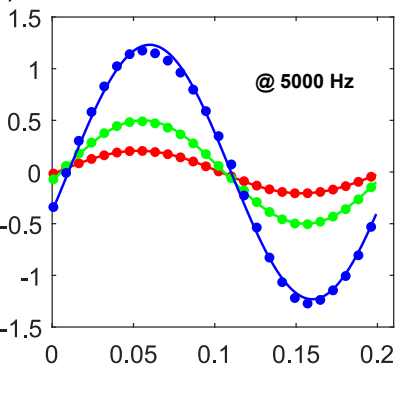

Figure A.8: Filtered and averaged polarization signal plotted with respect to time, for three different field amplitudes at a) $70 \mathrm{~Hz}$, b) $100 \mathrm{~Hz}$, c) $200 \mathrm{~Hz}$, d) $300 \mathrm{~Hz}$, e) $500 \mathrm{~Hz}$, f) $1000 \mathrm{~Hz}$, g) $2000 \mathrm{~Hz}, \mathrm{~h}) 300 \mathrm{~Hz}$ and i) $5000 \mathrm{~Hz}$. The markers represent the measured data, which includes all higher harmonics, and the solid lines are obtained by fitting with Eq. 5.9. 

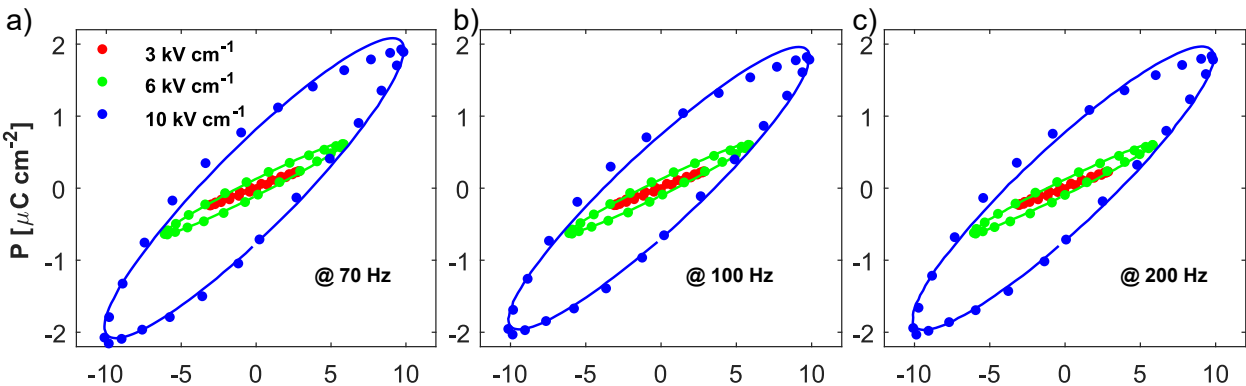

d)

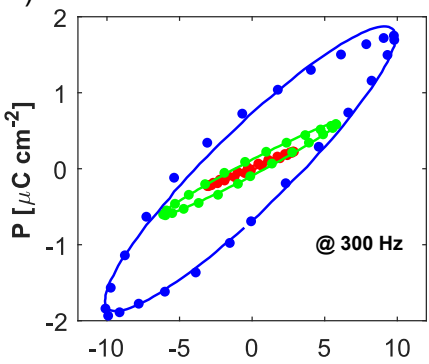

g)

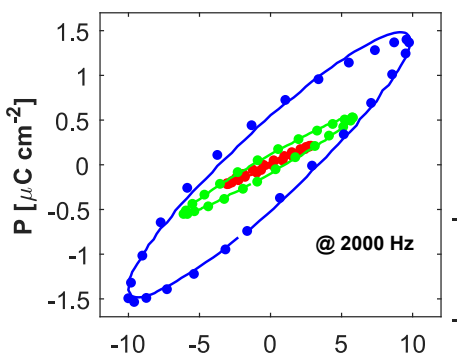

e)

$E[k V c m]$

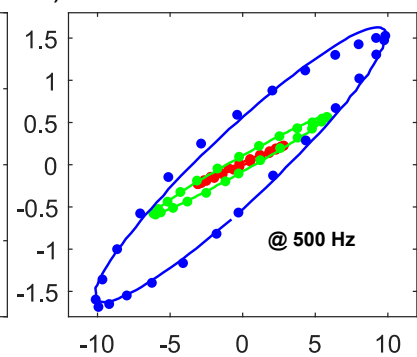

h)

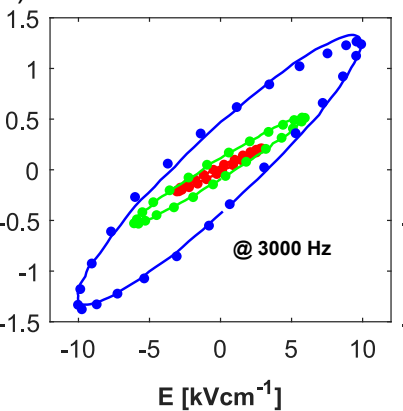

f)

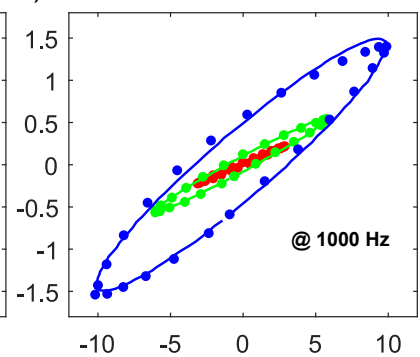

i)

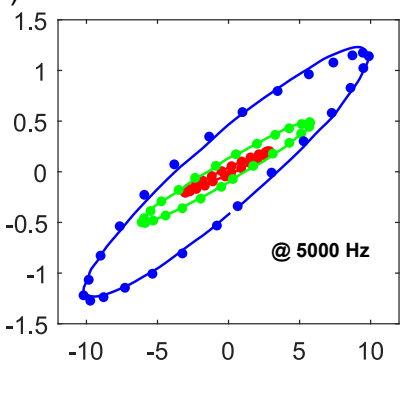

Figure A.9: Filtered and averaged polarization signal plotted with respect to field, for three different field amplitudes at a) $70 \mathrm{~Hz}$, b) $100 \mathrm{~Hz}$, c) $200 \mathrm{~Hz}$, d) $300 \mathrm{~Hz}$, e) $500 \mathrm{~Hz}$, f) $1000 \mathrm{~Hz}, \mathrm{~g}$ ) $2000 \mathrm{~Hz}, \mathrm{~h}) 300 \mathrm{~Hz}$ and i) $5000 \mathrm{~Hz}$. The markers represent the measured data, which includes all higher harmonics, and the solid lines are obtained by fitting with Eq. 5.9. 


\section{References}

[1] D. Damjanovic, Hysteresis in Piezoelectric and Ferroelectric Materials, in The Science of Hysteresis, edited by G. Bertotti and I. D. Mayergoyz (Academic Press, Oxford, 2006) Book section 4, pp. 337-465.

[2] D. Damjanovic and M. Demartin, The Rayleigh law in piezoelectric ceramics, Journal of Physics D: Applied Physics 29, 2057 (1996).

[3] D. A. Hall, Review Nonlinearity in piezoelectric ceramics, Journal of Materials Science 36, 4575 (2001).

[4] N. Bassiri-Gharb, I. Fujii, E. Hong, S. Trolier-Mckinstry, D. V. Taylor, and D. Damjanovic, Domain wall contributions to the properties of piezoelectric thin films, Journal of Electroceramics 19, 47 (2007).

[5] D. V. Taylor and D. Damjanovic, Evidence of domain wall contribution to the dielectric permittivity in PZT thin films at sub-switching fields, Journal of Applied Physics 82, 1973 (1997).

[6] D. V. Taylor, D. Damjanovic, and N. Setter, Nonlinear contributions to dielectric and piezoelectric properties in lead zirconate titanate thin films, Ferroelectrics 224, 299 (1999).

[7] D. V. Taylor, D. Damjanovic, E. Colla, and N. Setter, Fatigue and nonlinear dielectric response in sol-gel derived lead zirconate titanate thin films, Ferroelectrics 225, 91 (1999).

[8] D. Damjanovic and D. V. Taylor, Contributions to the nonlinear dielectric and piezoelectric response of ferroelectric thin films and ceramics, Ferroelectrics 221, 137 (1999).

[9] D. V. Taylor and D. Damjanovic, Piezoelectric properties of rhombohedral $\mathrm{Pb}(\mathrm{Zr}, \mathrm{Ti}) \mathrm{O}_{3}$ thin films with (100), (111), and "random" crystallographic orientation, Applied Physics Letters 76, 1615 (2000).

[10] A. Pramanick, D. Damjanovic, J. C. Nino, and J. L. Jones, Subcoercive Cyclic Electrical Loading of Lead Zirconate Titanate Ceramics I: Nonlinearities and Losses in the Converse Piezoelectric Effect, Journal of the American Ceramic Society 92, 2291 (2009).

[11] G. Robert, D. Damjanovic, and N. Setter, Piezoelectric hysteresis analysis and loss separation, Journal of Applied Physics 90, 4668 (2001).

[12] N. Bassiri-Gharb, S. Trolier-McKinstry, and D. Damjanovic, Piezoelectric nonlinearity in ferroelectric thin films, Journal of Applied Physics 100, 044107 (2006).

[13] S. Trolier-McKinstry, N. Bassiri Gharb, and D. Damjanovic, Piezoelectric nonlinearity due to motion of $180^{\circ}$ domain walls in ferroelectric materials at subcoercive fields: A dynamic poling model, Applied Physics Letters 88, 202901 (2006). 
[14] N. Bassiri-Gharb, S. Trolier-McKinstry, and D. Damjanovic, Strain-modulated piezoelectric and electrostrictive nonlinearity in ferroelectric thin films without active ferroelastic domain walls, Journal of Applied Physics 110, 124104 (2011).

[15] N. A. Pertsev, V. G. Kukhar, H. Kohlstedt, and R. Waser, Phase diagrams and physical properties of single domain epitaxial $\mathrm{PbZr}_{1-x} \mathrm{Ti}_{x} \mathrm{O}_{3}$ thin films, Physical Review B 67, 054107 (2003).

[16] E. P. Houwman, K. Vergeer, G. Koster, and G. Rijnders, Functional Properties of Polydomain Ferroelectric Oxide Thin Films, in Correlated Functional Oxides (Springer, 2017) Book section Chapter 2, pp. 29-53.

[17] E. Houwman, K. Vergeer, G. Koster, and G. Rijnders, Modelling functional properties of ferroelectric oxide thin films with a three-domain structure, arXiv preprint arXiv:1901.10883 (2019).

[18] D. Vanderbilt and M. H. Cohen, Monoclinic and triclinic phases in higher-order devonshire theory, Physical Review B 63, 094108 (2001).

[19] M. J. Haun, Z. Q. Zhuang, E. Furman, S. J. Jang, and L. E. Cross, Thermodynamic theory of the lead zirconate-titanate solid solution system, part III: Curie constant and sixth-order polarization interaction dielectric stiffness coefficients, Ferroelectrics 99, 45 (1989). 




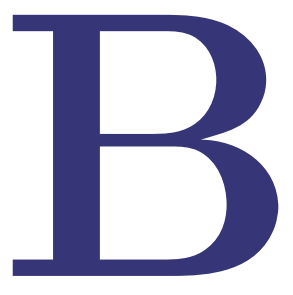

\section{Supporting Information Hysteresis, loss and nonlinearity in epitaxial $\mathrm{PbZr}_{0.55} \mathrm{Ti}_{0.45} \mathrm{O}_{3}$ films: Polarization rotation under a DC bias field}

\section{B.1 Amplitude of the polarization harmonics}

In the main manuscript it has been shown that the time dependent polarization response is given by Eq. 6.8 with the coefficients $c_{0}, c_{n, s}$, and $c_{n, c}$ that respectively represent the coefficients of the DC, sine and cosine terms where subscript $n$ represent the harmonic number. The number of harmonics and hence the number of $c$-coefficients depend on the AC field amplitude. In the following two subsections, explicit forms of the $c$-coefficients are given for amplitudes of 10 and $28 \mathrm{kV} \mathrm{cm}^{-1}$ for the case of non-zero bias of $20 \mathrm{kV} \mathrm{cm}^{-1}$ for which the $\beta$-coefficients were determined from fitting to the P-E loop. We will also show the relative amplitudes $P_{n, \mathrm{DC}} / P_{\mathrm{DC}}$ and how the normalized amplitudes $P_{n, \mathrm{DC}} / P_{1, \mathrm{DC}}$ that we measure compare to the normalized amplitudes that the model predicts for both cases at $3 \mathrm{kHz}$. 


\section{B.1.1 For $\mathrm{E}_{0}=10 \mathrm{kV} \mathrm{cm}^{-1}$ AC field amplitude}

The $c$-coefficient of the time independent term is:

$$
\begin{aligned}
c_{0} & =-E_{0}^{4}\left(\frac{\beta_{2}{ }^{2} \gamma_{P}{ }^{2} \omega^{2}}{4}+\frac{3 \beta_{2}{ }^{2}}{16}\right) \\
& -E_{0}{ }^{2}\left(\frac{\beta_{1}^{2} \gamma_{P}{ }^{2} \omega^{2}}{4}+\frac{\beta_{1}{ }^{2}}{4}+\frac{\beta_{2} \tan \left(\theta_{B}\right)}{2}\right)+1 \approx 1.00
\end{aligned}
$$

as can be found by inserting the values of the parameters, obtained from fitting the experimental data. Inserting this approximation into Eq. 6.8 in the main manuscript, it follows that all the hysteresis loops are centered around $P_{\mathrm{DC}}=$ $P_{S} \cos \left(\theta_{\mathrm{DC}, f}\right.$. This value can not be verified in the current experiment, as we are only able to measure the change of the polarization due to the applied AC field.

The coefficients of the time dependent harmonics are:

$$
\begin{aligned}
c_{1, s} & =-\beta_{1}\left[\left(\frac{\beta_{2} \gamma_{P}^{2} \omega^{2}}{2}+\frac{3 \beta_{2}}{4}\right) E_{0}{ }^{2}+\tan \left(\theta_{B}\right)\right] \\
c_{1, c} & =-\beta_{1} \gamma_{P} \omega\left[\frac{3 E_{0}^{2} \beta_{2}}{4}+\tan \left(\theta_{B}\right)\right] \\
c_{2, s} & =-\frac{E_{0}^{2} \beta_{2}^{2} \gamma_{P} \omega}{2}-\left(\frac{\gamma_{P} \omega \beta_{1}^{2}}{2}+\beta_{2} \gamma_{P} \omega \tan \left(\theta_{B}\right)\right) \\
c_{2, c} & =\frac{\beta_{2}^{2}}{4} E_{0}^{2}+\left(-\frac{\beta_{1}{ }^{2} \gamma_{P}{ }^{2} \omega^{2}}{4}+\frac{\beta_{1}^{2}}{4}+\frac{\beta_{2} \tan \left(\theta_{B}\right)}{2}\right) \\
c_{3, s} & =\left(\frac{\beta_{1} \beta_{2}}{4}-\frac{\beta_{1} \beta_{2} \gamma_{P}^{2} \omega^{2}}{2}\right) \\
c_{3, c} & =\frac{3 \beta_{1} \beta_{2} \gamma_{P} \omega}{4} \\
c_{4, s} & =\frac{\beta_{2}^{2} \gamma_{P} \omega}{4} \\
c_{4, c} & =\left(\frac{\beta_{2}^{2} \gamma_{P}^{2} \omega^{2}}{4}-\frac{\beta_{2}^{2}}{16}\right)
\end{aligned}
$$

The relative amplitudes $P_{n, \mathrm{DC}} / P_{\mathrm{DC}}=E_{0}^{n} \sqrt{c_{n, s}^{2}+c_{n, c}^{2}}$ of the harmonics that are tabulated in Tab. B.1 are calculated using a) the fitted values of $\theta(E)$ in Fig. $6.1 \mathrm{c})$ in the main manuscript, b) $E_{0}=10 \mathrm{kV} \mathrm{cm}^{-1}$, c) $f=3 \mathrm{kHz}$, d) $\gamma_{P}=$ $-3.27 \times 10^{-6} \mathrm{~Hz}^{-1} \mathrm{rad}^{-1}$ and e) $P_{\mathrm{DC}}=41 \mu \mathrm{C} \mathrm{cm}^{-2}$, for $E_{\mathrm{DC}}=20 \mathrm{kV} \mathrm{cm}^{-1}$.

We note that the rotation direction of the polarization hysteresis at each harmonic is counter clockwise (CCW) if the product $c_{n, s} \cdot c_{n, c}$ is negative, and clockwise (CW) when said product is positive. The model predicts that the odd harmonics rotate $\mathrm{CCW}$ and the even harmonics rotate $\mathrm{CW}$. The amplitude of the first harmonic is about $2 \%$ of the $\mathrm{DC}$ polarization, and for higher harmonics the relative amplitude rapidly decreases. 
Table B.1: The amplitudes of different harmonics at $10 \mathrm{kV} \mathrm{cm}^{-1}$.

\begin{tabular}{cccccc}
\hline $\boldsymbol{n}$ & $\mathbf{c}_{\mathbf{n}, \mathbf{s}}$ & $\mathbf{c}_{\mathbf{n}, \mathbf{c}}$ & Unit & Character & $\mathbf{P}_{\mathbf{n}} / \mathbf{P}_{\mathbf{D C}}$ \\
\hline 0 & - & - & - & Offset & 1 \\
1 & $1.7 \times 10^{-3}$ & $-1.1 \times 10^{-4}$ & $\left(\mathrm{kV} \mathrm{cm}^{-1}\right)^{-1}$ & $\mathrm{CCW}$ & $1.7 \times 10^{-2}$ \\
2 & $1.6 \times 10^{-6}$ & $1.3 \times 10^{-5}$ & $\left(\mathrm{kV} \mathrm{cm}^{-1}\right)^{-2}$ & $\mathrm{CW}$ & $1.3 \times 10^{-3}$ \\
3 & $-1.9 \times 10^{-8}$ & $3.55 \times 10^{-9}$ & $\left(\mathrm{kV} \mathrm{cm}^{-1}\right)^{-3}$ & $\mathrm{CCW}$ & $2.0 \times 10^{-5}$ \\
4 & $-1.6 \times 10^{-11}$ & $-6.3 \times 10^{-11}$ & $\left(\mathrm{kV} \mathrm{cm}^{-1}\right)^{-4}$ & $\mathrm{CW}$ & $6.8 \times 10^{-7}$ \\
\hline
\end{tabular}

We show a comparison of the measured amplitudes of the harmonics $P_{\text {meas }, n, \mathrm{DC}}$ and the predicted amplitudes of the harmonics $P_{n, \mathrm{DC}}$ in Fig. B.1. In this figure, the amplitudes are normalized to that of the first harmonic by usage of $P_{n, \mathrm{DC}} / P_{1, \mathrm{DC}}$. The determined $1 / f$ noise level is also indicated. The ratio of the measured value of the amplitude of the first harmonic to the polarization at the bias field is $P_{\text {meas, } 1, \mathrm{DC}} / P_{\mathrm{DC}}=1.4 \times 10^{-2}$, which is close to the value of $1.7 \times 10^{-2}$ that the model predicts. The measured amplitude of the $2^{\text {nd }}$ harmonic is smaller then the calculated value, whereas amplitudes of the $3 r d$ and $4^{\text {th }}$ harmonics are significantly higher than their predicted values. This behaviour was also observed for the zero bias case. [1] There it was speculated that the energy of the lower harmonics is transferred to the higher harmonics by a nonlinear process, which is expected to be the case here as well.

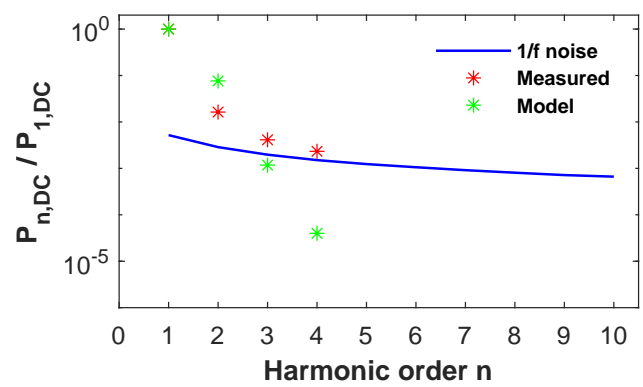

Figure B.1: Amplitude of $n^{\text {th }}$ harmonic normalized to the amplitude of the $1^{\text {st }}$ harmonic, as calculated from the model (green markers) and as measured (red markers) for an applied AC-field of $10 \mathrm{kV} \mathrm{cm}^{-1}$ at $3 \mathrm{kHz}$. The blue line indicates the measured $1 / f$ polarization noise. 


\section{B.1.2 For $\mathrm{E}_{0}=28 \mathrm{kV} \mathrm{cm}^{-1}$ AC field amplitude}

The time independent term is:

$$
\begin{aligned}
c_{0} & =\left(-\frac{5 \beta_{4}{ }^{2} \gamma_{P}{ }^{2} \omega^{2}}{16}-\frac{35 \beta_{4}{ }^{2}}{256}\right) E_{0}{ }^{8} \\
& +\left(-\frac{9 \beta_{3}^{2} \gamma_{P}^{2} \omega^{2}}{32}-\frac{5 \beta_{3}{ }^{2}}{32}-\frac{\beta_{2} \beta_{4} \gamma_{P}{ }^{2} \omega^{2}}{2}-\frac{5 \beta_{2} \beta_{4}}{16}\right) E_{0}{ }^{6} \\
& +\left(-\frac{\beta_{2}{ }^{2} \gamma_{P}{ }^{2} \omega^{2}}{4}-\frac{3 \beta_{2}^{2}}{16}-\frac{3 \beta_{1} \beta_{3} \gamma_{P}{ }^{2} \omega^{2}}{8}-\frac{3 \beta_{1} \beta_{3}}{8}-\frac{3 \beta_{4} \tan \left(\theta_{B}\right)}{8}\right) E_{0}{ }^{4} \\
& +\left(-\frac{\beta_{1}{ }^{2} \gamma_{P}{ }^{2} \omega^{2}}{4}-\frac{\beta_{1}{ }^{2}}{4}-\frac{\beta_{2} \tan \left(\theta_{B}\right)}{2}\right) E_{0}{ }^{2}+1 \approx 0.99 .
\end{aligned}
$$

Similar to $10 \mathrm{kV} \mathrm{cm}^{-1}$ case, the hysteresis loops are still centered around $P_{\mathrm{DC}}$.

$$
\begin{aligned}
c_{1, s} & =\left(-\frac{15 \beta_{3} \beta_{4} \gamma_{P}{ }^{2} \omega^{2}}{16}-\frac{35 \beta_{3} \beta_{4}}{64}\right) E_{0}{ }^{6} \\
& +\left(-\frac{5 \beta_{1} \beta_{4}}{8}-\frac{5 \beta_{2} \beta_{3}}{8}-\frac{\beta_{1} \beta_{4} \gamma_{P}{ }^{2} \omega^{2}}{2}-\frac{3 \beta_{2} \beta_{3} \gamma_{P}{ }^{2} \omega^{2}}{4}\right) E_{0}{ }^{4} \\
& +\left(-\frac{\beta_{1} \beta_{2} \gamma_{P}{ }^{2} \omega^{2}}{2}-\frac{3 \beta_{1} \beta_{2}}{4}-\frac{3 \beta_{3} \tan \left(\theta_{B}\right)}{4}\right) E_{0}{ }^{2}-\beta_{1} \tan \left(\theta_{B}\right) \\
c_{1, c} & =\left(-\frac{35 \beta_{3} \beta_{4} \gamma_{P} \omega}{64}\right) E_{0}{ }^{6}+\left(-\frac{5 \beta_{1} \beta_{4} \gamma_{P} \omega}{8}-\frac{5 \beta_{2} \beta_{3} \gamma_{P} \omega}{8}\right) E_{0}{ }^{4} \\
& +\left(-\frac{3 \beta_{3} \gamma_{P} \omega \tan \left(\theta_{B}\right)}{4}-\frac{3 \beta_{1} \beta_{2} \gamma_{P} \omega}{4}\right) E_{0}{ }^{2}-\beta_{1} \gamma_{P} \omega \tan \left(\theta_{B}\right) \\
c_{2, s} & =\left(-\frac{7 \beta_{4}{ }^{2} \gamma_{P} \omega}{16}\right) E_{0}{ }^{6}+\left(-\frac{15 \gamma_{P} \omega \beta_{3}{ }^{2}}{32}-\frac{15 \beta_{2} \beta_{4} \gamma_{P} \omega}{16}\right) E_{0}{ }^{4} \\
+ & \left(-\frac{\gamma_{P} \omega \beta_{2}{ }^{2}}{2}-\beta_{4} \gamma_{P} \omega \tan \left(\theta_{B}\right)-\beta_{1} \beta_{3} \gamma_{P} \omega\right) E_{0}{ }^{2}-\frac{\gamma_{P} \omega \beta_{1}{ }^{2}}{2}-\beta_{2} \gamma_{P} \omega \tan \left(\theta_{B}\right) \\
c_{2, c} & =\left(\frac{\beta_{4}{ }^{2} \gamma_{P}{ }^{2} \omega^{2}}{4}+\frac{7 \beta_{4}{ }^{2}}{32}\right) E_{0}{ }^{6}+\left(\frac{9 \beta_{3}{ }^{2} \gamma_{P}{ }^{2} \omega^{2}}{64}+\frac{15 \beta_{3}{ }^{2}}{64}+\frac{\beta_{2} \beta_{4} \gamma_{P}{ }^{2} \omega^{2}}{4}+\frac{15 \beta_{2} \beta_{4}}{32}\right) E_{0}{ }^{4} \\
& +\left(\frac{\beta_{2}{ }^{2}}{4}+\frac{\beta_{1} \beta_{3}}{2}+\frac{\beta_{4} \tan \left(\theta_{B}\right)}{2}\right) E_{0}{ }^{2}-\frac{\beta_{1}{ }^{2} \gamma_{P}{ }^{2} \omega^{2}}{4}+\frac{\beta_{1}{ }^{2}}{4}+\frac{\beta_{2} \tan \left(\theta_{B}\right)}{2}
\end{aligned}
$$




$$
\begin{aligned}
& c_{3, s}=\left(\frac{21 \beta_{3} \beta_{4}}{64}-\frac{3 \beta_{3} \beta_{4} \gamma_{P}^{2} \omega^{2}}{16}\right) E_{0}^{4} \\
& +\left(\frac{5 \beta_{1} \beta_{4}}{16}+\frac{5 \beta_{2} \beta_{3}}{16}-\frac{\beta_{1} \beta_{4} \gamma_{P}^{2} \omega^{2}}{4}-\frac{3 \beta_{2} \beta_{3} \gamma_{P}^{2} \omega^{2}}{8}\right) E_{0}{ }^{2} \\
& -\frac{\beta_{1} \beta_{2} \gamma_{P}^{2} \omega^{2}}{2}+\frac{\beta_{1} \beta_{2}}{4}+\frac{\beta_{3} \tan \left(\theta_{B}\right)}{4} \\
& c_{3, c}=\frac{63 \beta_{3} \beta_{4} \gamma_{P} \omega}{64} E_{0}{ }^{4}+\left(\frac{15 \beta_{1} \beta_{4} \gamma_{P} \omega}{16}+\frac{15 \beta_{2} \beta_{3} \gamma_{P} \omega}{16}\right) E_{0}{ }^{2} \\
& +\frac{3 \beta_{3} \gamma_{P} \omega \tan \left(\theta_{B}\right)}{4}+\frac{3 \beta_{1} \beta_{2} \gamma_{P} \omega}{4} \\
& c_{4, s}=\frac{7 \beta_{4}{ }^{2} \gamma_{P} \omega}{16} E_{0}{ }^{4}+\left(\frac{3 \gamma_{P} \omega \beta_{3}{ }^{2}}{8}+\frac{3 \beta_{2} \beta_{4} \gamma_{P} \omega}{4}\right) E_{0}{ }^{2} \\
& +\frac{\gamma_{P} \omega \beta_{2}^{2}}{4}+\frac{\beta_{4} \gamma_{P} \omega \tan \left(\theta_{B}\right)}{2}+\frac{\beta_{1} \beta_{3} \gamma_{P} \omega}{2} \\
& c_{4, c}=\left(\frac{\beta_{4}{ }^{2} \gamma_{P}{ }^{2} \omega^{2}}{4}-\frac{7 \beta_{4}{ }^{2}}{64}\right) E_{0}{ }^{4}+\left(\frac{9 \beta_{3}{ }^{2} \gamma_{P}{ }^{2} \omega^{2}}{32}-\frac{3 \beta_{3}{ }^{2}}{32}+\frac{\beta_{2} \beta_{4} \gamma_{P}{ }^{2} \omega^{2}}{2}-\frac{3 \beta_{2} \beta_{4}}{16}\right) E_{0}{ }^{2} \\
& +\frac{\beta_{2}^{2} \gamma_{P}{ }^{2} \omega^{2}}{4}-\frac{\beta_{2}^{2}}{16}+\frac{3 \beta_{1} \beta_{3} \gamma_{P}{ }^{2} \omega^{2}}{8}-\frac{\beta_{1} \beta_{3}}{8}-\frac{\beta_{4} \tan \left(\theta_{B}\right)}{8} \\
& c_{5, s}=\left(\frac{9 \beta_{3} \beta_{4} \gamma_{P}^{2} \omega^{2}}{16}-\frac{7 \beta_{3} \beta_{4}}{64}\right) E_{0}^{2}+\frac{\beta_{1} \beta_{4} \gamma_{P}^{2} \omega^{2}}{4}-\frac{\beta_{2} \beta_{3}}{16}-\frac{\beta_{1} \beta_{4}}{16}+\frac{3 \beta_{2} \beta_{3} \gamma_{P}^{2} \omega^{2}}{8} \\
& c_{5, c}=\left(-\frac{35 \beta_{3} \beta_{4} \gamma_{P} \omega}{64}\right) E_{0}^{2}-\frac{5 \beta_{1} \beta_{4} \gamma_{P} \omega}{16}-\frac{5 \beta_{2} \beta_{3} \gamma_{P} \omega}{16} \\
& c_{6, s}=\left(-\frac{3 \beta_{4}{ }^{2} \gamma_{P} \omega}{16}\right) E_{0}{ }^{2}-\frac{3 \gamma_{P} \omega \beta_{3}{ }^{2}}{32}-\frac{3 \beta_{2} \beta_{4} \gamma_{P} \omega}{16} \\
& c_{6, c}=\left(\frac{\beta_{4}^{2}}{32}-\frac{\beta_{4}^{2} \gamma_{P}^{2} \omega^{2}}{4}\right) E_{0}^{2}-\frac{9 \beta_{3}^{2} \gamma_{P}^{2} \omega^{2}}{64}+\frac{\beta_{3}^{2}}{64}-\frac{\beta_{2} \beta_{4} \gamma_{P}^{2} \omega^{2}}{4}+\frac{\beta_{2} \beta_{4}}{32} \\
& c_{7, s}=\frac{\beta_{3} \beta_{4}}{64}-\frac{3 \beta_{3} \beta_{4} \gamma_{P}^{2} \omega^{2}}{16} \\
& c_{7, c}=\frac{7 \beta_{3} \beta_{4} \gamma_{P} \omega}{64} \\
& c_{8, s}=\frac{\beta_{4}^{2} \gamma_{P} \omega}{32} \\
& c_{8, c}=\frac{\beta_{4}{ }^{2} \gamma_{P}{ }^{2} \omega^{2}}{16}-\frac{\beta_{4}{ }^{2}}{256}
\end{aligned}
$$


Table B.2: The amplitudes of different harmonics at $28 \mathrm{kV} \mathrm{cm}^{-1}$.

\begin{tabular}{cccccc}
\hline $\boldsymbol{n}$ & $\mathbf{c}_{\mathbf{n}, \mathbf{s}}$ & $\mathbf{c}_{\mathbf{n}, \mathbf{c}}$ & Unit & Character & $\mathbf{P}_{\mathbf{n}} / \mathbf{P}_{\mathbf{D C}}$ \\
\hline 0 & - & - & - & Offset & 0.99 \\
1 & $2.0 \times 10^{-3}$ & $-1.2 \times 10^{-4}$ & $\left(\mathrm{kV} \mathrm{cm}^{-1}\right)^{-1}$ & $\mathrm{CCW}$ & $5.6 \times 10^{-2}$ \\
2 & $1.6 \times 10^{-6}$ & $1.3 \times 10^{-5}$ & $\left(\mathrm{kV} \mathrm{cm}^{-1}\right)^{-2}$ & $\mathrm{CW}$ & $1.0 \times 10^{-2}$ \\
3 & $-1.2 \times 10^{-7}$ & $2.2 \times 10^{-8}$ & $\left(\mathrm{kV} \mathrm{cm}^{-1}\right)^{-3}$ & $\mathrm{CCW}$ & $2.7 \times 10^{-3}$ \\
4 & $-3.0 \times 10^{-10}$ & $-1.2 \times 10^{-9}$ & $\left(\mathrm{kV} \mathrm{cm}^{-1}\right)^{-4}$ & $\mathrm{CW}$ & $7.6 \times 10^{-4}$ \\
5 & $2.9 \times 10^{-12}$ & $-8.9 \times 10^{-13}$ & $\left(\mathrm{kV} \mathrm{cm}^{-1}\right)^{-5}$ & $\mathrm{CCW}$ & $5.2 \times 10^{-5}$ \\
6 & $5.6 \times 10^{-15}$ & $1.5 \times 10^{-14}$ & $\left(\mathrm{kV} \mathrm{cm}^{-1}\right)^{-6}$ & $\mathrm{CW}$ & $7.7 \times 10^{-6}$ \\
7 & $-8.9 \times 10^{-17}$ & $4.0 \times 10^{-17}$ & $\left(\mathrm{kV} \mathrm{cm}^{-1}\right)^{-7}$ & $\mathrm{CCW}$ & $1.3 \times 10^{-6}$ \\
8 & $-2.3 \times 10^{-19}$ & $-4.4 \times 10^{-19}$ & $\left(\mathrm{kV} \mathrm{cm}^{-1}\right)^{-8}$ & $\mathrm{CW}$ & $1.9 \times 10^{-7}$ \\
\hline & & & & &
\end{tabular}

As was observed for the case of lower field of $10 \mathrm{kV} \mathrm{cm}^{-1}$, the rotation direction of the harmonics is unchanged, as one would expect. All odd harmonics rotate CCW and all even harmonics rotate CW. The amplitude of the first harmonic is $5.6 \%$ of the polarization at the DC bias and is decreasing rapidly for the higher harmonics.

Fig. B.2 shows the measured and calculated normalized $P_{n, \mathrm{DC}} / P_{1, \mathrm{DC}}$ ratios. As was the case for the $10 \mathrm{kV} \mathrm{cm}^{-1}$ AC field amplitude, the ratio of the measured value $P_{\text {meas }, 1, \mathrm{DC}} / P_{\mathrm{DC}}=6.3 \times 10^{-2}$ is close to the predicted $5.6 \times 10^{-2}$ value and the measured amplitudes of the harmonics up to order 4 are smaller than the predicted values, whereas the measured values of the higher harmonics are significantly higher then predicted. Also here we speculate that this is due to a nonlinear mechanism that transfers energy from the lower harmonics to the higher harmonics, which is not taken into account in the model.

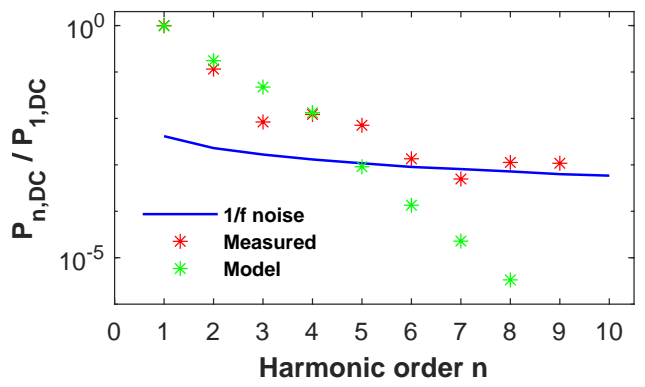

Figure B.2: Amplitude of $n^{\text {th }}$ harmonic normalized to the amplitude of the $1^{\text {st }}$ harmonic, as calculated from the model (green markers) and as measured (red markers) for an applied field of $28 \mathrm{kV} \mathrm{cm}^{-1}$ at $3 \mathrm{kHz}$. The blue line indicates the measured $1 / f$ polarization noise. 


\section{B.2 Area calculation for the polarization hysteresis loops}

The area of the polarization loop is described by integrating the multiplication of the time dependent polarization response with the time dependent applied field. In the following example, the loop area is calculated for the case of a polarization signal described by a polynomial of order $p=2$ for $\Delta \theta(E)$ and it will be shown that only the first order harmonic contributes to the area. The same calculation holds true for polynomials with higher orders.

$$
\begin{aligned}
A_{P} & =\left|\int_{c y c l e} P(E) d E\right| \\
& =\left|\int_{0}^{2 \pi} P_{\mathrm{DC}}\left[c_{0}+\sum_{n=1}^{4} c_{n, s} E_{0}^{n} \sin (n \omega t)+c_{n, c} E_{0}^{n} \cos (n \omega t)\right] \cdot E_{0} \omega \cos (\omega t) d t\right| \\
& =\mid P_{\mathrm{DC}}[\underbrace{\int_{0}^{2 \pi} c_{0} E_{0} \omega \cos (\omega t) d t}_{=0} \\
& +\int_{0}^{2 \pi}\{\underbrace{c_{1, s} E_{0} \sin (\omega t) \cdot E_{0} \omega \cos (\omega t)}_{=0}+\underbrace{c_{1, c} E_{0} \cos (\omega t) \cdot E_{0} \omega \cos (\omega t)}_{=0}\} d t \\
& +\int_{0}^{2 \pi}\{\underbrace{c_{2, s} E_{0}^{2} \sin (2 \omega t) \cdot E_{0} \omega \cos (\omega t)}_{=0}+\underbrace{c_{2, c} E_{0}^{2} \cos (2 \omega t) \cdot E_{0} \omega \cos (\omega t)}_{=0}\} d t \\
& +\int_{0}^{2 \pi}\{\underbrace{c_{3, s} E_{0}^{3} \sin (3 \omega t) \cdot E_{0} \omega \cos (\omega t)}_{=0}+\underbrace{c_{3, c} E_{0}^{3} \cos (3 \omega t) \cdot E_{0} \omega \cos (\omega t)}_{=0}\} d t \\
& +\int_{0}^{2 \pi}\{\underbrace{c_{\mathrm{DC}}^{c_{4, s} E_{0}^{4} \sin (4 \omega t) \cdot E_{0} \omega \cos (\omega t)}+\underbrace{c_{4, c} E_{0}^{4} \cos (4 \omega t) \cdot E_{0} \omega \cos (\omega t)}_{=0}\} d t}_{=0}]
\end{aligned}
$$




\section{B.3 Strain hysteresis loops at different frequencies}

Fig. B.3 and Fig. B.4 display, respectively in time and field domain, the filtered and averaged strain signals for all measured frequencies other than $3 \mathrm{kHz}$. As in the main manuscript also here only every fifth data point is shown for clarity. The slopes and opening of the strain hysteresis loops in Fig. B.4 are proportional to $d_{33, f}$ and $\gamma_{S}$, respectively. As can be seen, the slopes for different amplitudes are the same within the error-bars, as is discussed in the main manuscript, and the loop openings are very small. The model fits that are shown with solid lines are perfectly matching the experimental data.
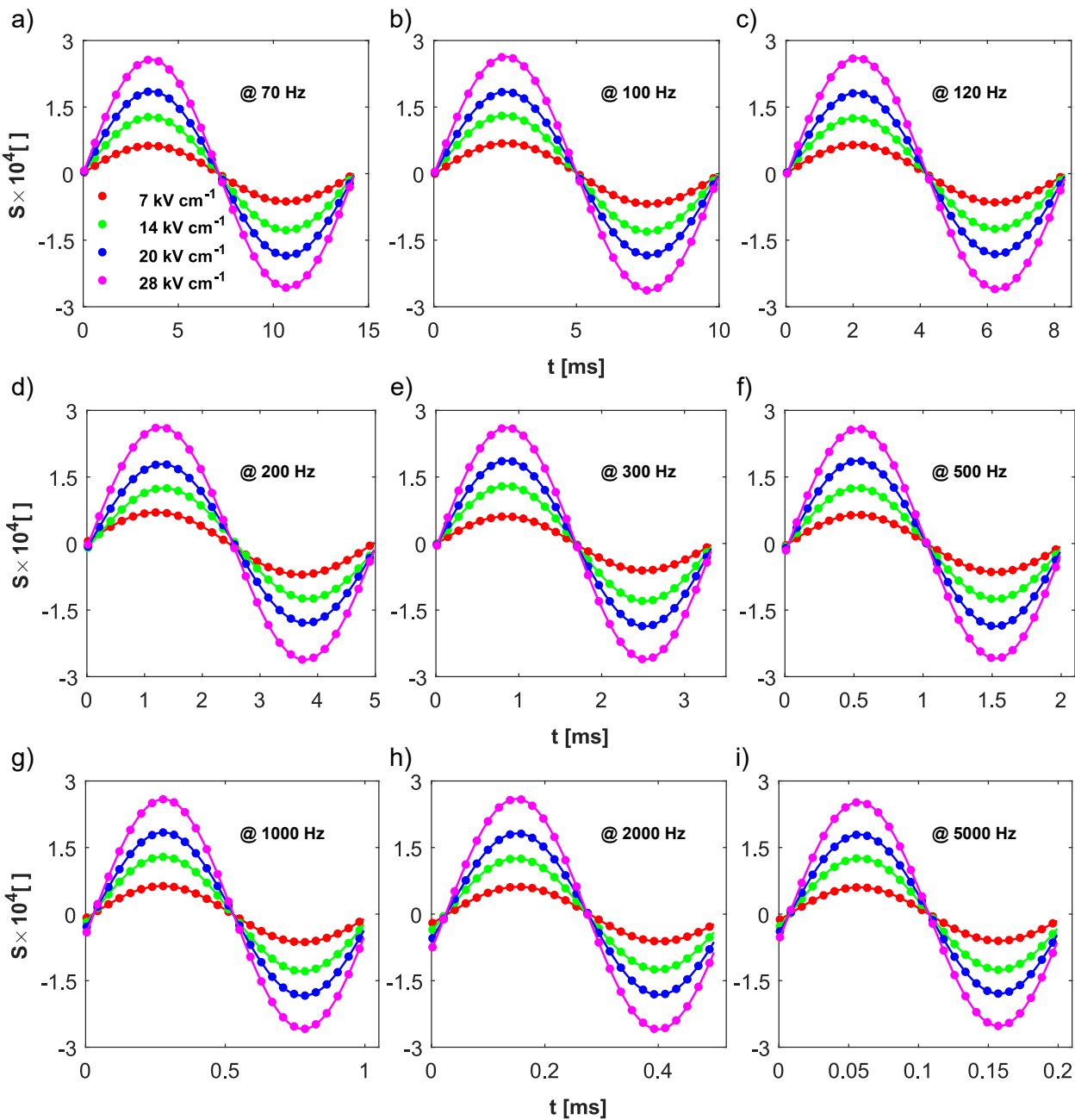

i)

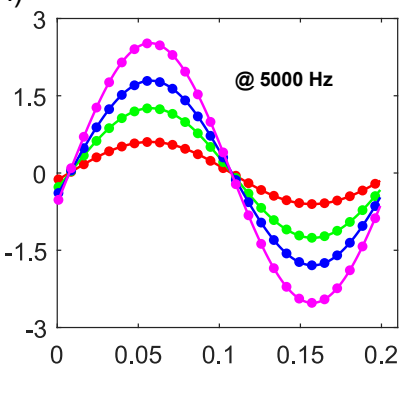

Figure B.3: Filtered and averaged strain signal plotted against time at a bias of $20 \mathrm{kV} \mathrm{cm}^{-1}$ at a) $70 \mathrm{~Hz}$, b) $100 \mathrm{~Hz}$, c) $120 \mathrm{~Hz}$,d) $200 \mathrm{~Hz}$, e) $300 \mathrm{~Hz}$, f) $500 \mathrm{~Hz}$, g) $1000 \mathrm{~Hz}, \mathrm{~h}) 2000 \mathrm{~Hz}$ and i) $5000 \mathrm{~Hz}$. Datapoints show the measurements and the lines are obtained from fitting with Eq. 6.3 main manuscript. 
a)

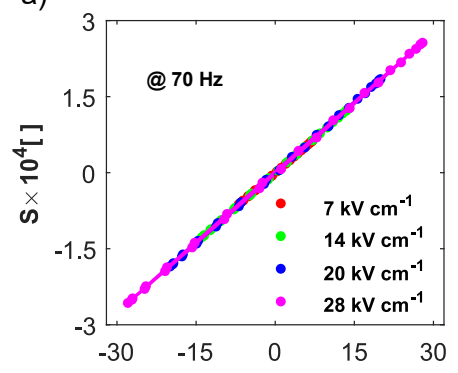

d)

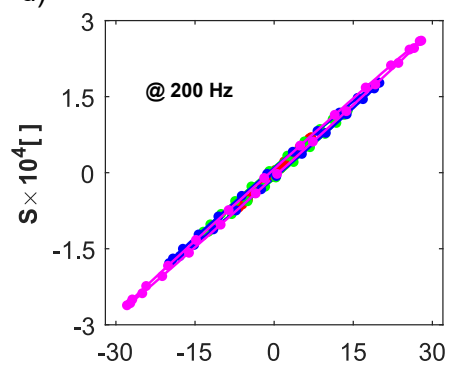

g)

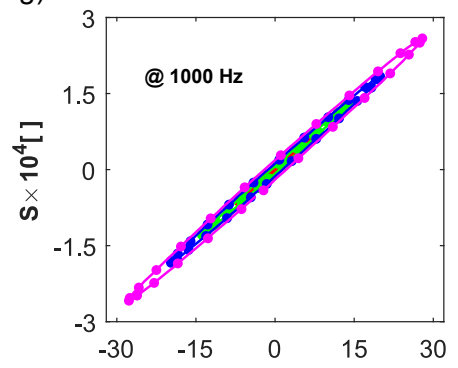

b)

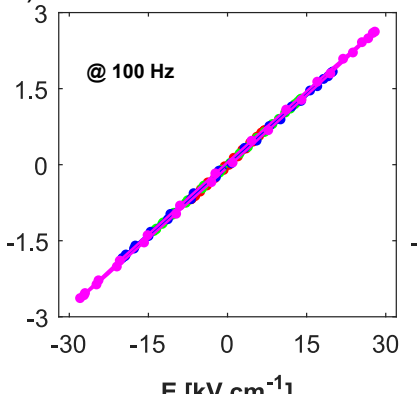

e)

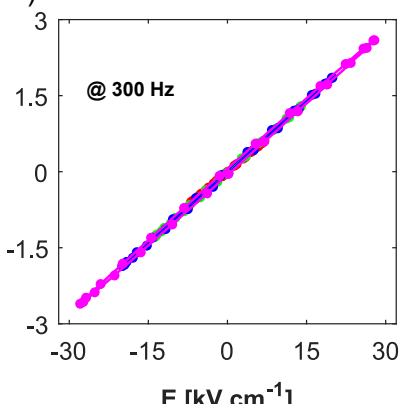

h)

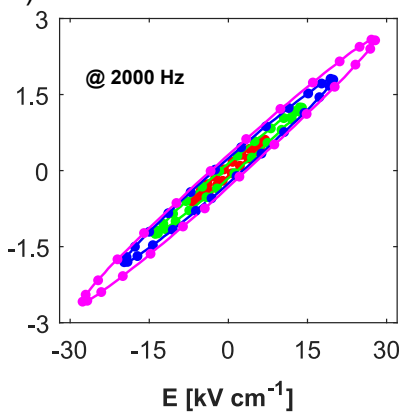

c)

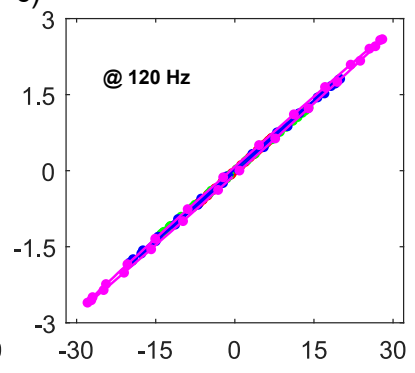

f)

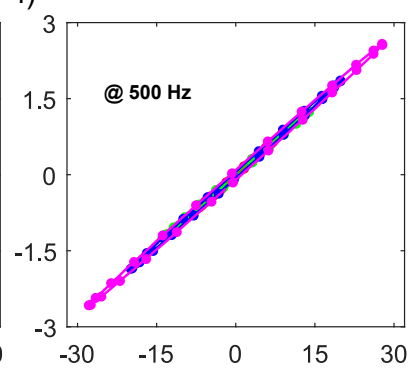

i)

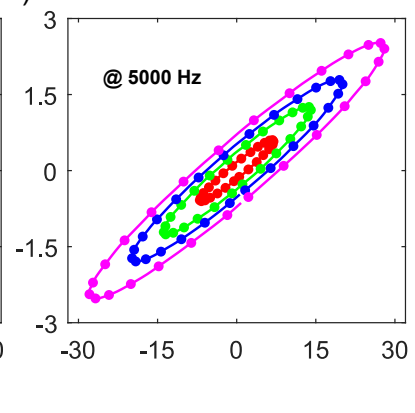

Figure B.4: Filtered and averaged strain signal plotted against $\mathrm{AC}$ electric field at a bias of $20 \mathrm{kV} \mathrm{cm}^{-1}$ at a) $70 \mathrm{~Hz}$, b) $100 \mathrm{~Hz}$, c) $120 \mathrm{~Hz}$, d) $200 \mathrm{~Hz}$, e) $300 \mathrm{~Hz}$, f) $500 \mathrm{~Hz}$, g) $1000 \mathrm{~Hz}, \mathrm{~h}$ ) $2000 \mathrm{~Hz}$ and i) $5000 \mathrm{~Hz}$. Data points show the measurements and the lines are obtained from fitting with Eq. 6.3 in the main manuscript. 


\section{B.4 Polarization hysteresis loops at different fre- quencies}

Fig. B.5 and Fig. B.6 show, respectively in time and field domain, the polarization hysteresis loops for all measured frequencies other than $3 \mathrm{kHz}$. The data points were obtained by bandpass filtering at all harmonic frequencies and then averaged over the measured 20 cycles. The standard deviation of the average is less than the marker size. All higher harmonics are included in the experimental loops. One can clearly observe the elliptical shape of the hysteresis loops at all frequencies and amplitudes. The experimental loops were fitted with the fundamental harmonic only, depicted by the continuous lines in the figures. The fits show that the contribution of the higher harmonics are only noticeable at the extremes of the AC-field cycle for the highest field amplitude. 
a)

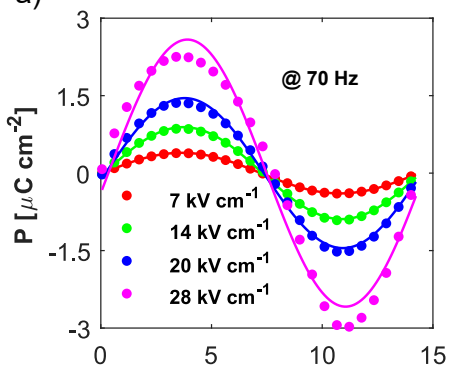

d)

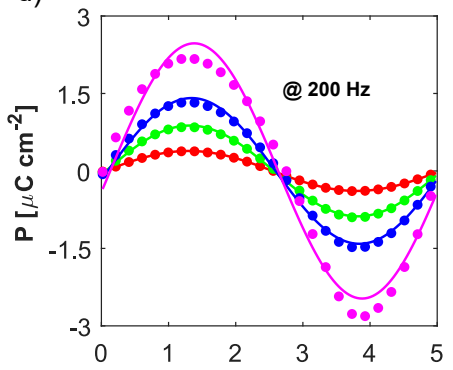

g)

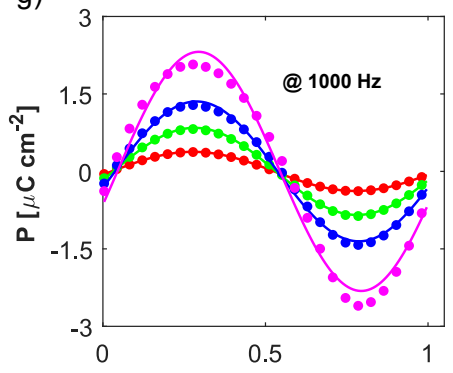

b)

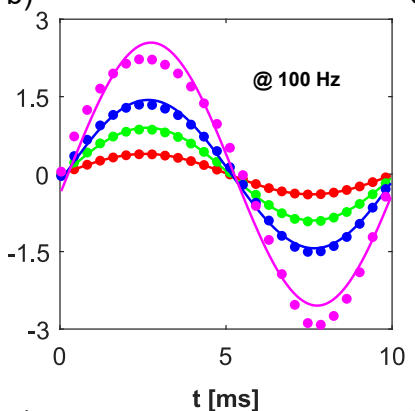

e)

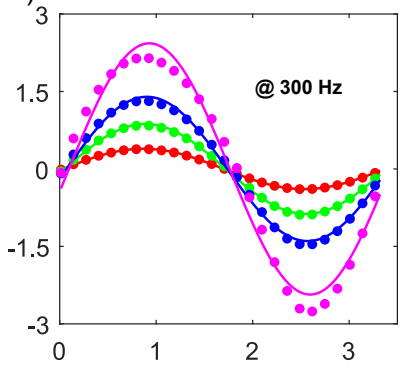

c)

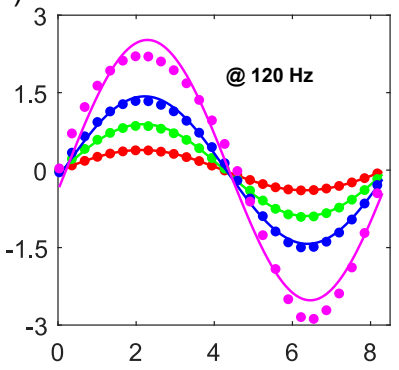

h)

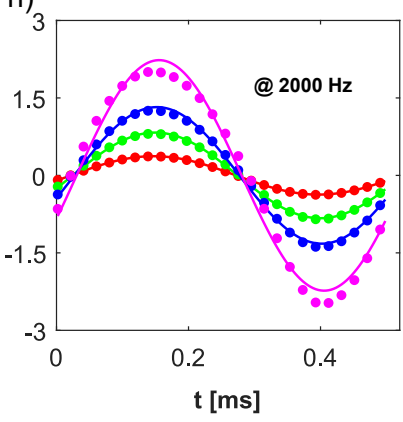

f)

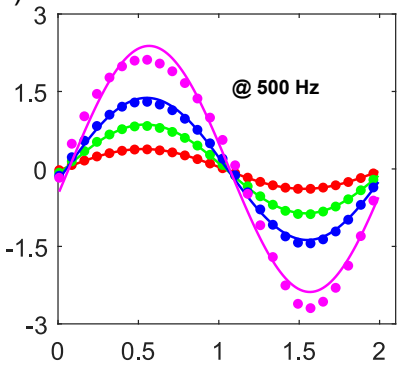

i)

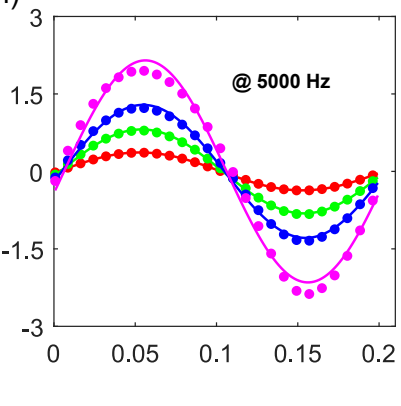

Figure B.5: Filtered and averaged polarization signal plotted with respect to time at a bias of $20 \mathrm{kV} \mathrm{cm}^{-1}$ at a) $70 \mathrm{~Hz}$, b) $100 \mathrm{~Hz}$, c) $120 \mathrm{~Hz}$, d) $200 \mathrm{~Hz}$, e) $300 \mathrm{~Hz}$, f) $500 \mathrm{~Hz}$, g) $1000 \mathrm{~Hz}, \mathrm{~h}$ ) $2000 \mathrm{~Hz}$ and i) $5000 \mathrm{~Hz}$. The markers represent the measured data, which includes all higher harmonics, and the solid lines are obtained by fitting with Eq. 6.13 main manuscript. 
a)

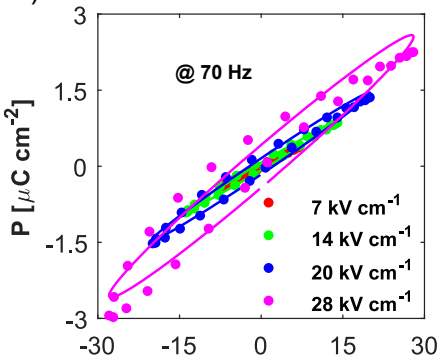

d)

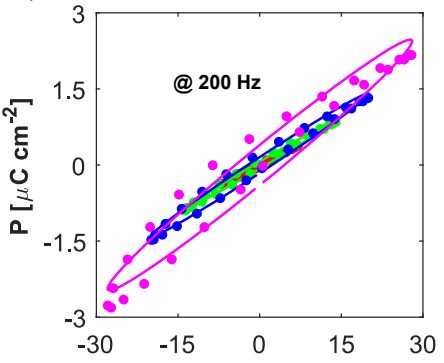

g)

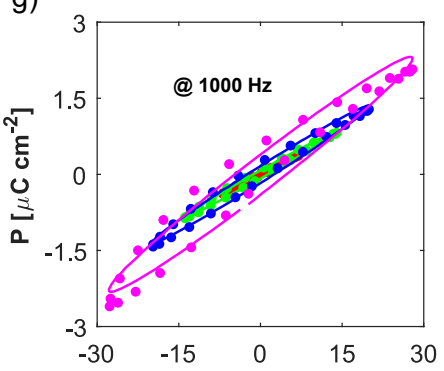

b)

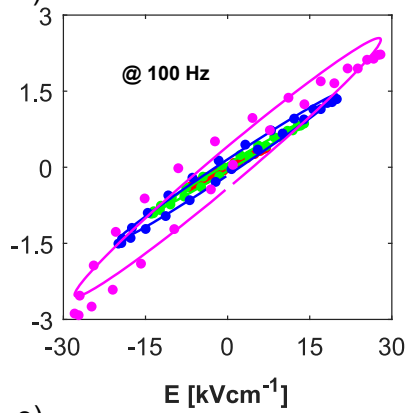

e)

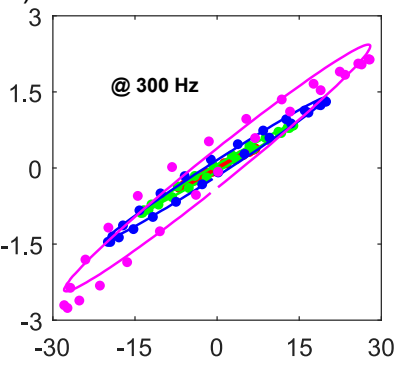

h)

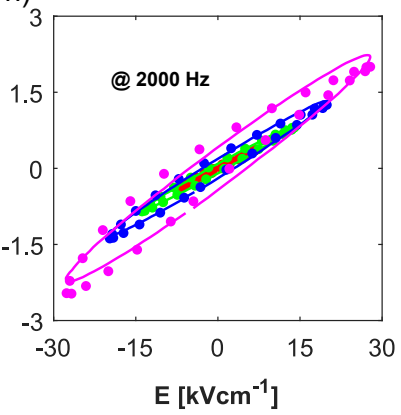

c)

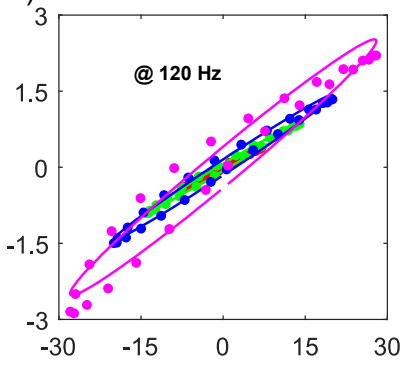

f)

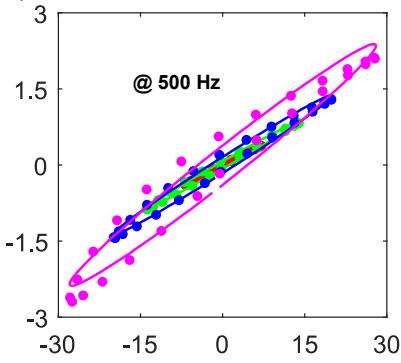

i)

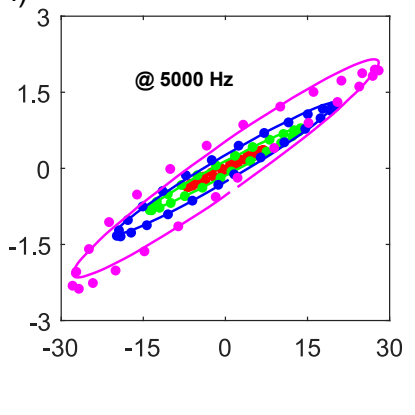

Figure B.6: Filtered and averaged polarization signal plotted with respect to electric field, at a bias of $20 \mathrm{kV} \mathrm{cm}^{-1}$ at a) $70 \mathrm{~Hz}$, b) $100 \mathrm{~Hz}$, c) $120 \mathrm{~Hz}$, d) $200 \mathrm{~Hz}$, e) $300 \mathrm{~Hz}$, f) $500 \mathrm{~Hz}$, g) $1000 \mathrm{~Hz}$, h) $2000 \mathrm{~Hz}$ and i) $5000 \mathrm{~Hz}$. The markers represent the measured data, which includes all higher harmonics, and the solid lines are obtained by fitting with Eq. 6.13 main manuscript. 


\section{B.5 Fitting of the polarization loss tangent}

As described in the main manuscript, the frequency dependence of the polarization loss tangent is fitted with $\tan \delta_{p}\left(E_{0}, \omega\right)=\tan \delta_{0}^{*}\left(E_{0}\right)+\left|\gamma_{P} \omega\right|$, where $\tan \delta_{0}^{*}$ is a free fitting parameter depending on the applied AC field amplitude. A single $\gamma_{p}$ value is obtained from simultaneous fitting of all curves by least-square fitting. To extract $\tan \delta_{0}$ and $c$ the resulting $\tan \delta_{0}^{*}(E)$ values are fitted with $\tan \delta_{0}^{*}\left(E_{0}\right)=c E_{0}^{2}+\tan \delta_{0}$. Fig. B.7 a) and b) show the fits for the zero bias case, for the frequency dependence and the field dependence of $\tan \delta_{0}^{*}$ respectively. The fits describe the experimental data very well. Fig. B.7c) shows the field dependence of $\tan \delta_{0}^{*}$ for the non-zero bias case. Is is seen that the fit with a second order field dependence fits the data reasonably good. A linear fit would describe that data better, however then the loss mechanism would change for the non-zero bias case, which we think is not reasonable.

a)

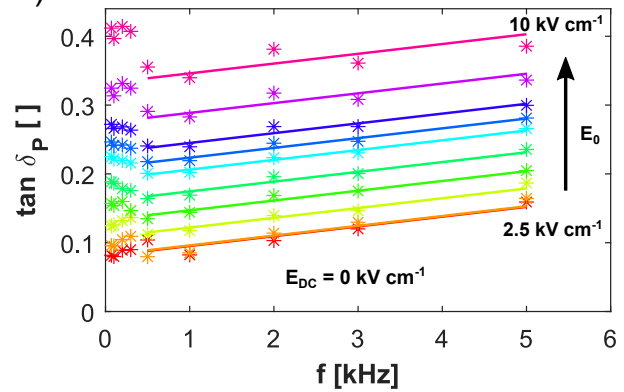

b)

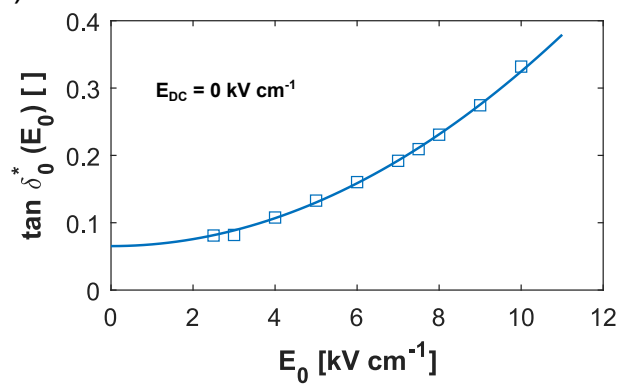

c)

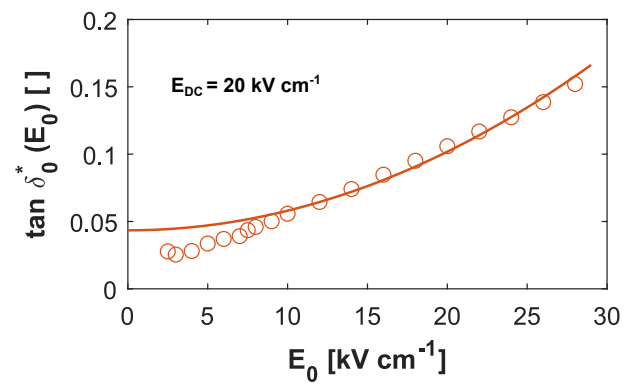

Figure B.7: a) The polarization loss tangent as function of frequency for varying field amplitudes at zero bias. The extracted $\tan \delta_{0}^{*}\left(E_{0}\right)$ for a) zero bias case and b) $20 \mathrm{kV} \mathrm{cm}^{-1}$ bias case. Lines denote the fit. 


\section{References}

[1] P. Lucke, M. Bayraktar, Y. A. Birkhölzer, M. Nematollahi, A. Yakshin, G. Rijnders, F. Bijkerk, and E. P. Houwman, Hysteresis, Loss and Nonlinearity in Epitaxial $\mathrm{PbZr}_{0.55} \mathrm{Ti}_{0.45} \mathrm{O}_{3}$ Films: A Polarization Rotation Model, Advanced Functional Materials, 2005397 (2020). 




\section{Summary}

Piezoelectric thin films are of interest for modern MEMS systems, for example used in applications like energy harvesters, actuators, adaptive optics or inkjetprinter heads. This thesis describes physics studies on the growth and modelling of $\mathrm{PbZr}_{0.52} \mathrm{Ti}_{0.48} \mathrm{O}_{3}$ (PZT) thin films. It contains a new description of hysteresis, loss and nonlinearity for the sub-coercive field strain and polarization response for epitaxial PZT thin films.

The thesis consists of three parts. The first part presents a small-scale demonstrator of an adaptive XUV optic based on nanoscale piezoelectric surface manipulation. The demonstrator consists of a PZT thin film with an array of top electrodes to selectively control the actuation. It is shown that by utilizing a resistive layer on top of the piezoelectric layer, it is possible to achieve a controlled, gradual surface profile between the electrodes. The resulting deformations range in the nm range and are laterally scalable from sub $\mathrm{mm}$ to the $\mathrm{mm}$ range, which makes it suitable for corrections of optics in the XUV range. To our knowledge this is the first presentation of a gradual surface modulation utilizing thin film piezoelectrics.

The second part describes the influence of a $\mathrm{LaNiO}_{3}$ (LNO) template layer on the growth and the functional properties of the PZT film subsequently grown on the LNO. We show that by tuning the deposition conditions, oxygen pressure and thickness of the template layer, the growth of the PZT film can be changed from a smooth dense film into a rough columnar film. This change of morphology changes the ferroelectric properties of the films and is explained by an increase of the roughness of the template layer to accommodate strain relaxation. The results clearly underline the importance of the template layer for the growth and the functional properties of PZT thin films.

The third part of the thesis introduces a new model, the so-called polarization rotation model, to describe the hysteresis, loss and nonlinearity in epitaxial PZT thin films of monoclinic and rhombohedral crystal symmetry. The model is based on the rotation of the polarization vector within the unit cell, which is accompanied by a viscous interaction of the domains. In contrast to the widely used Rayleigh model, the polarization rotation model takes the crystal symmetry of the material into account. It is shown that for monoclinic PZT films the experimentally observed strain and polarization response can not be described by the Rayleigh model, but is perfectly described by the polarization rotation model. Furthermore, the new model differentiates between the nonlinearity of the response and the loss due to viscous interaction. In addition, it is shown that by addition of a non-zero bias term to the model, it also describes the measured strain and polarization response for a 
DC bias of $20 \mathrm{kV} \mathrm{cm}^{-1}$, explaining the observed reduction of loss and nonlinearity of the polarization response. 


\section{Samenvatting}

Piezoelektrische dunne lagen zijn erg interessant voor moderne MEMS systemen, bijvoorbeeld in toepassingen als energy harvesters, actuatoren, adaptieve optische systemen of ink-jet printerkoppen. In dit proefschrift wordt de groei en de fysische modellering van $\mathrm{PbZr}_{0.52} \mathrm{Ti}_{0.48} \mathrm{O}_{3}$ (PZT) dunne lagen beschreven. Het bevat een nieuwe beschrijving van de hysterese, hysterese verlies en niet-lineaire respons van de strain (mechanische spanning) en de polarisatie in epitaxiale PZT dunne lagen voor veldsterkten met een waarde die lager is dan het coercieve veld.

Het proefschrift omvat drie delen. In het eerste deel wordt een klein-schalig demonstratie model van een adaptieve XUV optiek gepresenteerd, dat gebaseerd is op de manipulatie in het nanometer bereik van het spiegelopppervlak door piezoelektrische elementen. Dit demo-model bestaat uit een PZT dunne laag met een matrix rij van top elektrodes voor de selectieve aansturing van de piezoelektrische activering. Het wordt aangetoond dat, door gebruik te maken van een resistieve tussenlaag boven op de PZT laag, het mogelijk is om op een controleerbare wijze een gradueel veranderend oppervlakteprofiel tussen de elektrodes te realiseren. De uiteindelijke verticale vervorming is in het bereik van nanometers en is in het vlak van de dunne laag te schalen van minder dan een millimeter tot enkele millimeters. Daarmee is het geschikt voor de correctie van optiek in het golflengtebereik van XUV optiek. Voor zover ons bekend is, is dit de eerste keer dat graduele oppervlakte modulatie met behulp van een piezoelektrische dunne laag wordt gedemonstreerd.

Het tweede deel beschrijft het effect van een $\mathrm{LaNiO}_{3}$ (LNO) 'template' laag op de groei en de functionele eigenschappen van de PZT laag, die bovenop deze laag wordt gegroeid. Door de depositie -condities, zuurstofdruk en de dikte van de LNOlaag aan te passen, kan de groei van de PZT laag worden veranderd van een gladde, dichte laag naar een ruwe laag die bestaat uit kolommen. Deze verandering van de morfologie verandert de ferroelektrische eigenschappen van de laag. Dit wordt verklaard door de verandering van de ruwheid van de template laag, wat weer veroorzaakt wordt door relaxatie van de opgebouwde mechanische spanningen in de LNO laag. Deze resultaten tonen het belang aan van de template laag voor de groei en functionele eigenschappen van PZT dunne lagen.

In het derde deel van dit proefschrift wordt een nieuw fysisch model geïntroduceerd, het zogenaamde polarisatie-rotatie model, dat de hysterese, het hysteretisch energieverlies en het niet-lineaire gedrag van epitaxiale PZT lagen met een monokliene of rhombohedrische kristalsymmetrie beschrijft. Het model is gebaseerd op de oscillerende rotatie van de polarisatievector in de eenheidscel onder invloed van een oscillerend elektrisch veld. Deze rotatie gaat samen met een visceuse interactie tussen de verschillende polarisatiedomeinen. In tegenstelling tot het normaliter 
gebruikte Rayleigh model, bepaalt in het polarisatie-rotatie model de kristalsymmetrie het gedrag. Het wordt aangetoond dat in monokliene PZT lagen de experimenteel bepaalde spanning en polarisatie respons niet kan worden beschreven met het Rayleigh model, maar wel heel goed met het polarisatie-rotatie model. Bovendien maakt dit model het mogelijk om onderscheid te maken tussen niet-lineaire respons enerzijds en het hysteretisch verlies-gedrag door visceuse interacties anderzijds. Tenslotte wordt aangetoond dat door toevoeging van een extra term aan het model, die een DC-elektrisch veld beschrijft, ook de gemeten spanning en polarisatie respons van een PZT laag onder een additioneel DC bias veld van $20 \mathrm{kV} \mathrm{cm}^{-1} \mathrm{kan}$ worden beschreven, waardoor de gemeten reductie van het hystereseverlies en de niet-lineariteit van de polarisatierespons kan worden uitgelegd. 


\section{Acknowledgments}

After 4 Years of very interesting and diverse research work the long journey of my $\mathrm{PhD}$ is at the end. Here I want to express my gratitude to all the people who allowed me to succeed in my research and without whom I would not have accomplished this work.

First I would like to express my deepest gratitude to my supervisor Fred Bijkerk for giving me the opportunity to start my $\mathrm{PhD}$ in the Industrial Focus Group XUV Optics and for his encouragement and guidance on the way of my PhD. Thank you for the many discussions and for keeping me on the right path.

I am deeply indepted to my Co-Supervisors Andrey Yakshin and Muharrem Bayraktar for their support and patience. Andrey, thank you for keeping me on track, for your calm words when I pressured myself too much and for your constructive criticism. Muharrem, thank you for all of our discussions on hysteresis and PLD growth, for the discussion on the experimental setups, for all the very constructive criticism and your many pointers on writing papers and programming with Matlab. For all of this I am deeply grateful.

This research would not have been possible without the input from Benjamin Wylie van Eerd and Mohammadreza Nemantollahi who both supported me as Post Docs in the project. Thank you for helping with the design of experiments, for teaching me the ins and outs of thin film deposition and the many discussions we had on both work and not work related topics.

My research also benefited from the insight of external members. For the many discussions on the materials science part of the research I would like to sincerely thank Guus Rijnders and Evert Houwman. Without your input and supervision the work on hysteresis would not have been possible.

Many thanks go especially to Minh Nguyen for his help and patience in showing me some of his secrets on PLD depositions and for introducing me to all ferro- and piezoelectric characterization methods. I sincerely thank Yorick Birkhölzer for all of the help and for teaching me XRD measurements and for his friendship and all of our discussions on our research which always proved to give a new perspective. I want to extend my gratefullness to the members of my graduation committee Beatriz Noheda, Emmanuel Dafay, Wilfred van der Wiel, André ten Elshof and Evert Houwman for reading my thesis, providing valuable feedback and the insightful discussion. I am also grateful for the many discussions with the members of our project team from the ZEISS SMT side. Especially I would like to thank Kerstin Hild, Toralf Gruner and Jörn Weber for the many fruitful discussions and exchanges in our regular meetings.

I am very grateful for all the technical support, especially from Frank Roesthuis and Dominic Post. Thank you for all the time you spent to introduce me to the equipment or to fix the PLD.

I would also like to thank all other senior members of the XUV team, Eric Louis, 
Robbert van den Kruijs, Marko Sturm, Igor Makhotkin and Wesley van den Beld for the valuable discussions during and outside of all the meetings.

I would like to sincerely thank Jacqueline Emmerich and Carin Krijnen-Smid for all the help in the administrative issues.

Special thanks go to Mark Smithers for helping me obtain the SEM crossections that I needed many times during my research. They were always of top quality and I enjoyed our discussions and talks very much.

I would like to thank my colleagues from the XUV group for the friendly working atmosphere, especially to Airat Shafikov, Niels Schukkink, Theo van Oijen, Denis Ijpes, Anirudhan Chandrasekaran, Roman Medvedev and Seda Kizir, for all the encouragement and the always nice discussions and the fun we had at coffee breaks and group events.

I thank the members of the IMS group for all their practical help when working in the lab and the many discussions we had and for all the fun we had when playing football.

I would also like to thanks the members of the DIOK badminton association for the many nice evenings, be it training, the association evening or any events. It was always a good way to free my head after a long day of work.

To the end I would like to express my deepest gratitude to my family for all your support, trust and confidence in me. Whenever I was feeling down you put me back on my feet.

Last but not least I would like to thank my partner Maike who was responsible for me starting a PhD in Twente. Thank you for suggesting to me to look for a $\mathrm{PhD}$ position at your old university. But more importantly thank you for your support, and your strength. You always had my back during the past years, no matter what. 


\section{Curriculum Vitae}

Philip Lucke was born on $25^{\text {th }}$ of February 1990 in Moers, Germany. After finishing his school education in Moers, he moved to Bochum to study physics at the RuhrUniversität Bochum (RUB). He obtained his bachelor's degree on the topic of "Nonequilibrium-supra conduction: $\mathrm{S}-$ and $\mathrm{D}$ - wave described by a $T^{*}$ and $\mu^{*}$ model". For his master's thesis he worked at the Institute of Experimental Plasma Physics II of the RUB on atmospheric-pressure-plasmas under the supervision of Prof. Dr. Jan Benedikt. He worked on the fabrication and characterization of Si- and $\mathrm{SiC}$ nano particles. During this time he spent time at the group of Prof. Dr. Davide Mariotti at the Nanotechnology and Integrated Bioengineering Centre of Ulster University in Northern Ireland. After finishing his Master successfully in 2016 he pursued a $\mathrm{PhD}$ in the Netherlands at the University of Twente in the Industrial Focus Group XUV Optics. Under his promotor Prof. dr. Fred Bijkerk and under the supervision of Dr. Andrey Yakshin and Dr. Muharrem Bayraktar he investigated the growth and the effects of hysteresis, loss and nonlinearity of epitaxial PZT thin films. Thereby he gained experience in experimental thin film growth, especially pulsed laser deposition and also on the topic of ferroelectric measurements and the theory of ferroelectrics. During this time he contributed to peer-reviewed journal publications and conference presentations, some of which can be found in this thesis. Currently he works in the Industrial Focus Group XUV Optics using his expertise on ferro- and piezoelectric thin films in the PIN3S project. 



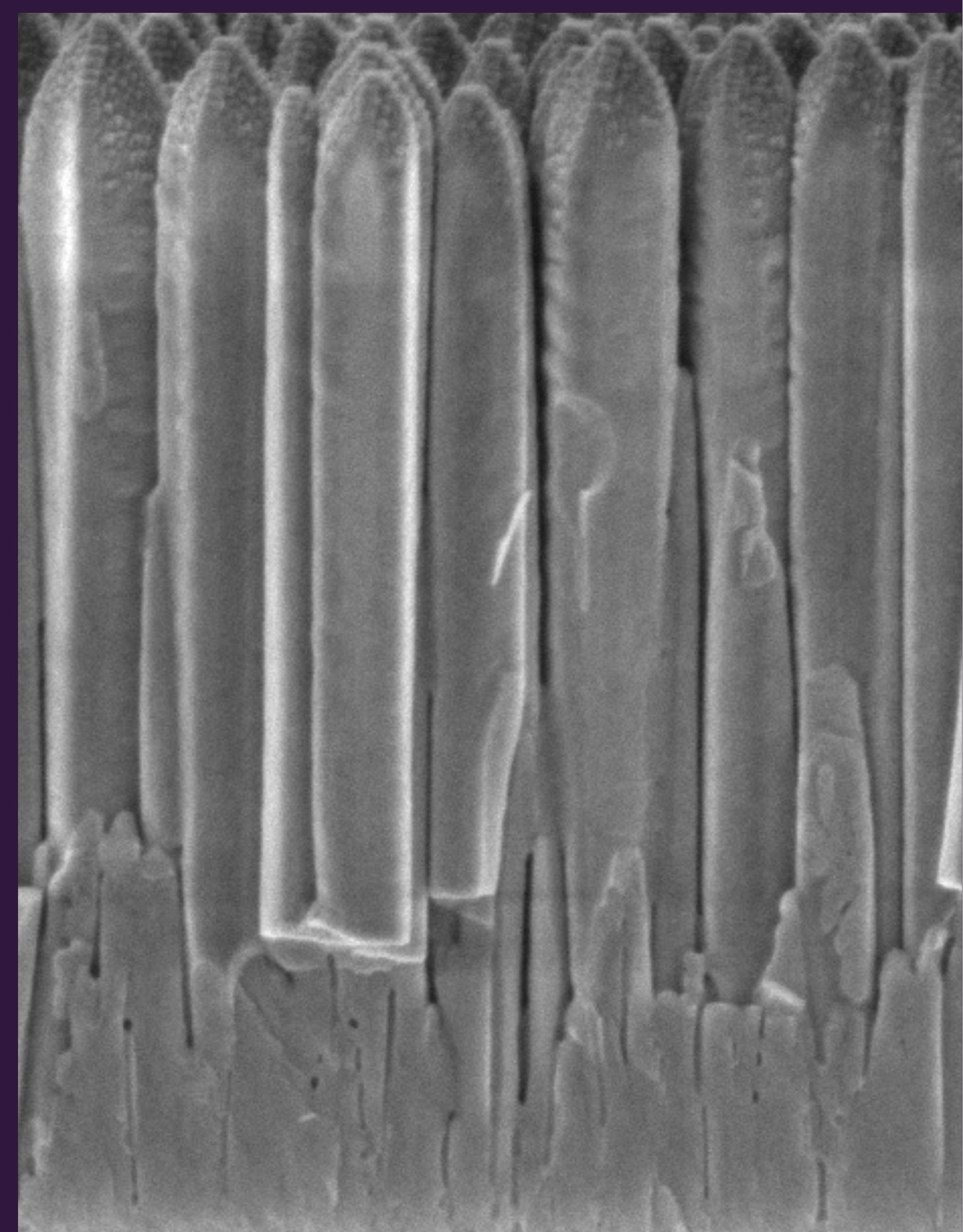

ISBN: $978-90-365-5152-6$ 